\title{
Atomic scale characterization and surface chemistry of metal modified titanate nanotubes and nanowires
}

\author{
Ákos Kukovecz ${ }^{\mathrm{a}, \mathrm{b}}$, Krisztián Kordás ${ }^{\mathrm{c}}$, János Kiss ${ }^{\mathrm{d}, \mathrm{e}, *}$, Zoltán Kónyaa,e,* \\ ${ }^{a}$ Department of Applied and Environmental Chemistry, MTA-SZTE “Lendület” Porous Nanocomposites Research Group, University of Szeged, Hungary \\ ${ }^{\mathrm{b}}$ MTA-SZTE "Lendület" Porous Nanocomposites Research Group, University of Szeged, Hungary \\ ${ }^{c}$ Microelectronics and Materials Physics Laboratories, EMPART Research Group of Infotech Oulu, University of Oulu, PL 4500 FIN-90014 Oulu, Finland \\ ${ }^{\mathrm{d}}$ Department of Physical Chemistry and Materials Science, University of Szeged, Hungary \\ ${ }^{\mathrm{e}}$ MTA-SZTE Reaction Kinetics and Surface Chemistry Research Group, University of Szeged, Hungary
}

Received 7 March 2016; received in revised form 2 June 2016; accepted 6 June 2016

Available online 23 June 2016

\begin{abstract}
Titanates are salts of polytitanic acid that can be synthesized as nanostructures in a great variety concerning crystallinity, morphology, size, metal content and surface chemistry. Titanate nanotubes (open-ended hollow cylinders measuring up to $200 \mathrm{~nm}$ in length and $15 \mathrm{~nm}$ in outer diameter) and nanowires (solid, elongated rectangular blocks with length up to $1500 \mathrm{~nm}$ and 30-60 nm diameter) are the most widespread representatives of the titanate nanomaterial family. This review covers the properties and applications of these two materials from the surface science point of view. Dielectric, vibrational, electron and X-ray spectroscopic results are comprehensively discussed first, then surface modification methods including covalent functionalization, ion exchange and metal loading are covered. The versatile surface chemistry of onedimensional titanates renders them excellent candidates for heterogeneous catalytic, photocatalytic, photovoltaic and energy storage applications, therefore, these fields are also reviewed.
\end{abstract}

(c) 2016 Elsevier B.V. All rights reserved.

Keywords: Titania; Titanates; Oxide surfaces; Semiconductors; Metal nanoparticles; Fermi energy

\section{Contents}

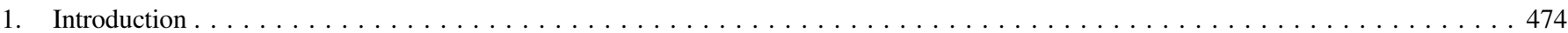

2. Synthesis, surface structure and properties of low dimensional titanates $\ldots \ldots \ldots \ldots \ldots \ldots$

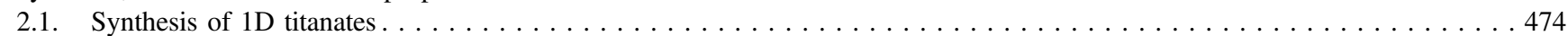

2.2. Crystal and microstructure of $1 \mathrm{D}$ titanates and their conversions $\ldots \ldots \ldots \ldots \ldots$

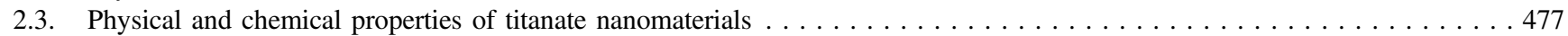

3. Surface and near surface analysis of protonated nanowires and nanotubes $\ldots \ldots \ldots \ldots 77$

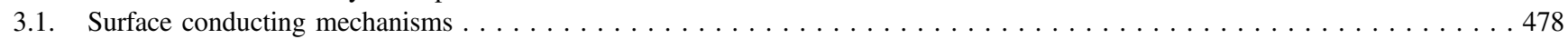

3.1.1. Dependence of charge transport processes on the amount of adsorbed water $\ldots \ldots \ldots 478$

3.1.2. Dependence of the activation energy on water adsorption $\ldots \ldots \ldots \ldots \ldots \ldots$

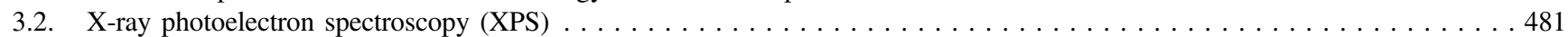

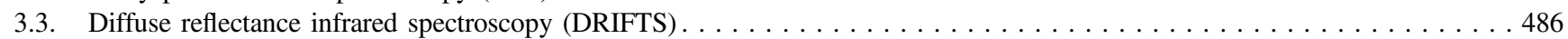

3.4. Raman spectroscopy on $\mathrm{H}$-form titanate nanotubes and nanowires $\ldots \ldots \ldots \ldots \ldots$

\footnotetext{
*Corresponding authors at: MTA-SZTE Reaction Kinetics and Surface Chemistry Research Group, University of Szeged, Hungary.
}

E-mail addresses: jkiss@chem.u-szeged.hu (J. Kiss), konya@chem.u-szeged.hu (Z. Kónya). 
3.5. Optical properties of $\mathrm{H}$-form titanate nanowires and nanotubes; $\mathrm{UV} / \mathrm{vis}$ spectrometry $\ldots \ldots \ldots \ldots$

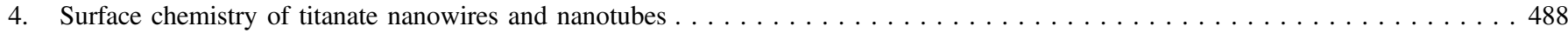

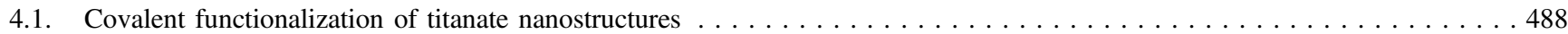

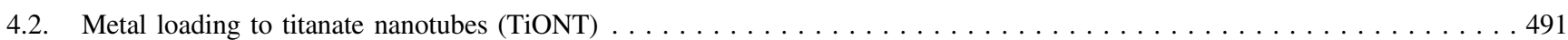

4.2.1. Effect of ion exchange on the surface properties of titanate nanostructures $\ldots \ldots \ldots \ldots 1$

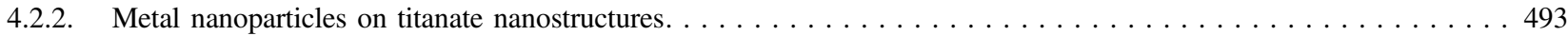

4.3. Phase stability and phase transformation of titanate nanostructures upon metal loading $\ldots \ldots \ldots \ldots$

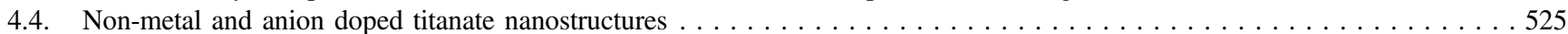

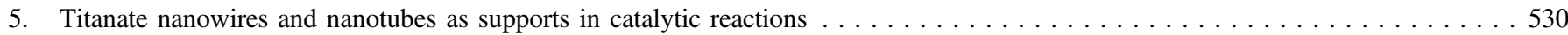

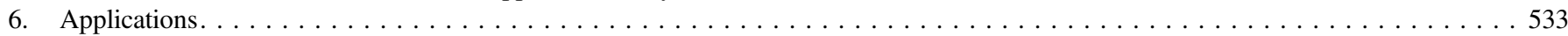

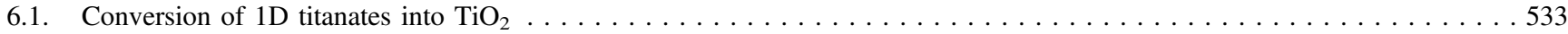

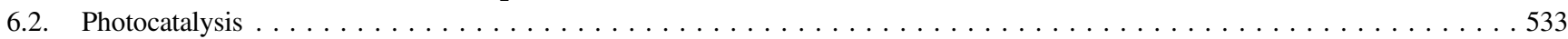

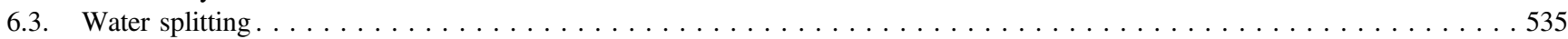

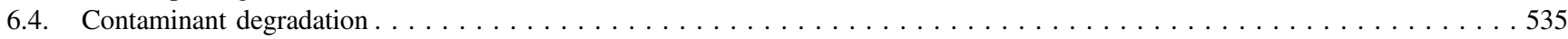

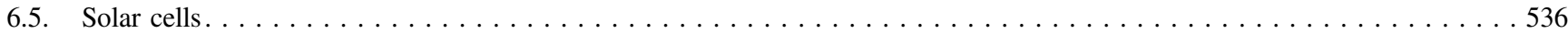

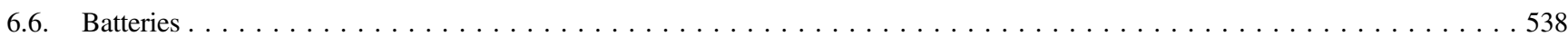

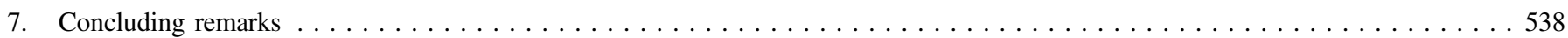

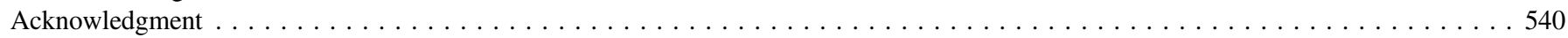

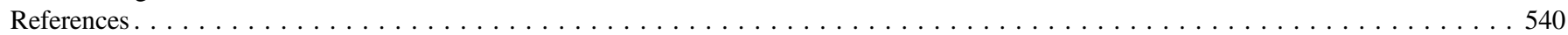

\section{Introduction}

Research interest in the area of polytitanate-based nanostructures has been increasing steadily in the past decade. The family of polytitanate-based metal titanates features a layered structure, with each layer consisting of parallel zig-zag ribbons, in which $\mathrm{TiO}_{6}$ octahedra share edges at one level in linear groups of three and are further joined together by corners. These ribbons share the terminal corners with identical ribbons forming an open octahedral framework enclosing tunnels or continuous rows of interstitial positions, in which the metal ions (generally $\mathrm{H}^{+}, \mathrm{Na}^{+}$or $\mathrm{K}^{+}$) are situated. The resulting nanostructures are usually nanotubes or nanowires, although structures featuring less (short nanorods) or even more (flat nanosheets) anisotropy are also possible. The typical length, outer and inner diameter of titanate nanotubes is 50$200 \mathrm{~nm}, 8-15 \mathrm{~nm}$ and $4-8 \mathrm{~nm}$, respectively. They have a hollow spiral cross-section and they are open at both ends. Titanate nanowires are solid objects with a quasi-rectangular cross-section and characteristic length and diameter of 300$1500 \mathrm{~nm}$ and 30-60 nm, respectively [1] (Fig. 1). Titanate nanostructures must be clearly distinguished from $\mathrm{TiO}_{2}$-based particles because the two have considerably different physical and chemical properties.

A broad literature coverage and excellent reviews are available on $\mathrm{TiO}_{2}$ nanostructures [2-4], perovskites [5] and anodically oxidized vertically oriented free standing $\mathrm{TiO}_{2}$ nanotube arrays [6,7]. However, a detailed account of the surface properties of polytitanate-based layered nanostructures is missing from the literature even though titanate nanotubes and nanowires are investigated in an increasing number of surface science related applications, e.g. heterogeneous catalysis and energy storage. In this paper we fill this gap by presenting a comprehensive review of the surface science of one-dimensional titanate nanomaterials. Their typical synthesis and most important physico-chemical properties will be summarized first. The next section details their surface properties by a comprehensive discussion of dielectric, XP, DRIFT, Raman and UV-vis spectroscopic results. Based on this knowledge, surface chemistry is explored next: covalent functionalization, metal loading, phase transformations and other surface-related phenomena are reviewed. The last two sections cover the emerging applications of one-dimensional titanate nanomaterials. They are currently investigated as heterogeneous catalytic supports, photocatalysts and energy generation/storage materials [8].

\section{Synthesis, surface structure and properties of low dimensional titanates}

\subsection{Synthesis of $1 D$ titanates}

Layered titanate nanomaterials are produced today almost exclusively by the alkaline hydrothermal synthesis discovered in 1998 by Kasuga et al. [9]. This method uses readily available materials $\left(\mathrm{TiO}_{2}, \mathrm{NaOH}\right.$, water) and offers good nanomaterial yield plus the option to control the product morphology by tuning the composition of the reaction mixture.

In a typical titanate nanotube synthesis $2 \mathrm{~g}$ of anatase $\mathrm{TiO}_{2}$ is mixed into $140 \mathrm{ml} 10 \mathrm{M} \mathrm{NaOH}$ aqueous solution until a white suspension is obtained. The suspension is then aged in a closed, unperturbed Teflon-lined autoclave at $130{ }^{\circ} \mathrm{C}$ for $72 \mathrm{~h}$. The product is washed with deionized water to reach $\mathrm{pH} 8$ at which point the slurry is filtered and the titanate nanotubes are dried in air. There has been some ambiguity in the literature concerning the exact crystalline phase produced by this procedure because the XRD profiles of several titanates are very similar and spontaneuous phase changes (e.g. during storage) are also possible. Nevertheless, it is safe to say that the first product of the alkaline hydrothermal synthesis reaction is a layered nanostructured titanate material that is well approximated as sodium trititanate $\left(\mathrm{Na}_{2} \mathrm{Ti}_{3} \mathrm{O}_{7}\right)$. 


\section{Nomenclature}

IRAS Infrared reflexion absorption spectroscopy

TiONT (TiNT) titanate nanotubes

TiONW (TiNW) titanate nanowires

ML monolayer

$E_{g} \quad$ band gap energy

$E_{F} \quad$ Fermi energy

DFT density functional theory

UHV ultra-high vacuum

XPS X-ray photoelectron spectroscopy

FTIR Fourier transform infrared spectroscopy

DRIFTS (FTIR) diffuse reflectance infrared Fourier transform spectroscopy

UV-vis ultraviolet-visible spectroscopy

AFM atomic force microscopy

STM scanning tunneling microscopy
TEM transmission electron microscopy
HRTEMhigh resolution transmission electron microscopy
SEM scanning electron microscopy
XRD X-ray diffraction
EDS energy dispersive X-ray spectroscopy
NMR nuclear magnetic resonance
ED electron diffraction
LFD low frequency dispersive (process)
LEIS low energy ion scattering spectroscopy
MIEC mixed ionic-electronic conduction
EXAFS extended X-ray absorption fine structure
PVD physical vapor deposition
CVD chemical vapor deposition
LSPR localized surface plasmonic resonance
1D one dimensional
2D two dimensional

Titanate nanowires are synthesized similarly, since titanate nanotubes are actually intermediates in the $\mathrm{TiO}_{2}$ to titanate nanowire recrystallization process. The same experimental setup and reaction mixture should be used, but the process must be
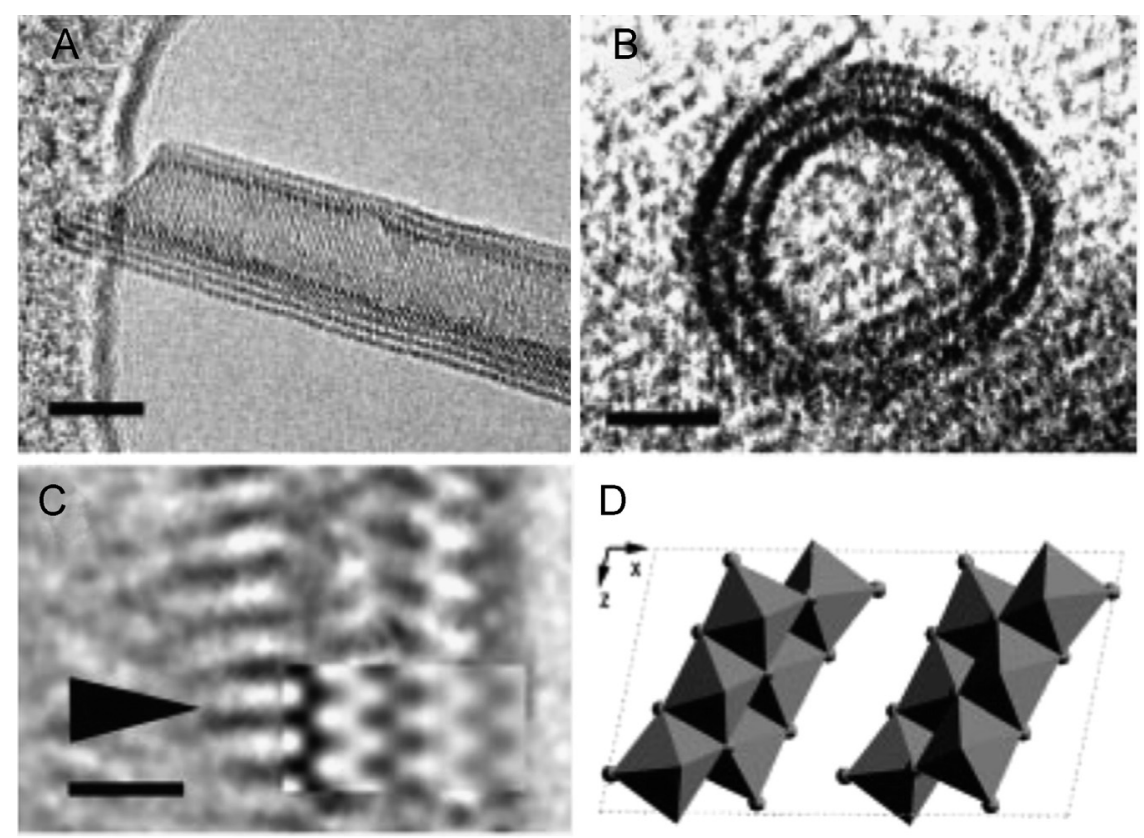

E
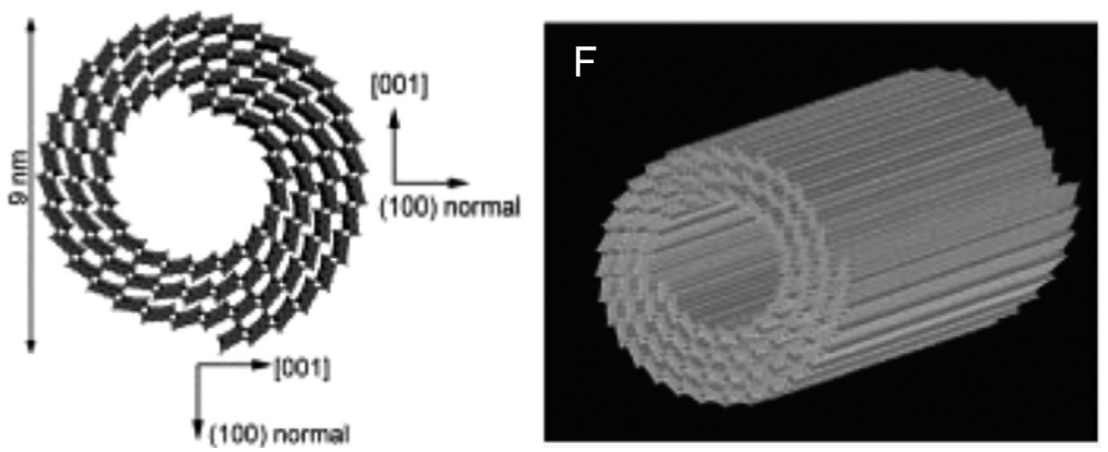

Fig. 1. Titanate nanotubes: (a), (b) HRTEM image; (c) enlarged HRTEM image of a part in (a); (d) structure model of one unit cell of $\mathrm{H}_{2} \mathrm{Ti}_{3} \mathrm{O}_{7}$ on the [010] projection; (e) schematic drawing of the structure of nanotubes; and (f) three-dimensional drawing of a nanotube. Reproduced from Ref. [1]. 
intensified by either increasing the reaction temperature to approx. $180{ }^{\circ} \mathrm{C}$ or increasing the reaction time to approx. 1 week or agitating the system to eliminate diffusion limitations.

Layered anisotropic titanate nanostructures can be prepared from a broad range of $\mathrm{TiO}_{2}$ sources [10-12] and using different compositions, bases [13-15], reaction times, temperatures and post-synthetic treatments. Changing the recipe allows fine-tuning the properties of the product mixture (e.g. nanotube:nanowire ratio, diameter and length distribution) [16-23].

The Kasuga process relies pressurized equipment, which can adversely affect the scalability of the method. Bavykin et al. were able to overcome this issue by refluxing $\mathrm{TiO}_{2}$ in an aqueous mixture of $\mathrm{NaOH}$ and $\mathrm{KOH}$ for $48 \mathrm{~h}$ at $100{ }^{\circ} \mathrm{C}$. Titanate nanotubes were obtained this way, and the product distribution was tunable by changing the reaction conditions [24].

Titanate nanostructures can be converted into each other [25]. Thermal treatment induces the transformation of the tritianate structure into $\mathrm{TiO}_{2}-\mathrm{B}\left(400{ }^{\circ} \mathrm{C}\right)$, anatase $\left(700{ }^{\circ} \mathrm{C}\right)$ and then rutile $\left(1000{ }^{\circ} \mathrm{C}\right)$. The open inner channel of nanotubes is preserved up to $400{ }^{\circ} \mathrm{C}$, then the structure gradually collapses into shorter nanorods [26-29]. Nanowires can withstand temperatures up to $1000{ }^{\circ} \mathrm{C}$ without the loss of their fibrous morphology, then they convert into more isotropic fragments [30]. Although nanowires are thermodynamically favored over nanotubes, it is also possible to convert nanowires back into nanotubes by non-equilibrium processes like mechanochemical activation [31].

\subsection{Crystal and microstructure of $1 D$ titanates and their conversions}

Titanates are salts of polytitanic acids, in which $\mathrm{TiO}_{6}$ octahedra represent the basic building blocks of various kinds of lattices. The charge neutrality of the lattice is ensured by cations of alkali and alkali-earth metals and/or protons. The way how the $\mathrm{TiO}_{6}$ octahedra are connected each other and then organize into nanometer size structures determine the crystal and microstructure of the nanomaterials. Tri and tetra-titanic acid crystals are built from edge sharing octahedra with three and four repeating units, respectively. These materials crystallize in monoclinic lattice. Rolling the (100) plane around the [010] or [001] axes tubular structures are obtained [1]. Rolling several of those, the structure becomes layered. On the other hand, in dititanic crystals only two repeating units are in edge sharing position and form orthorhombic lattice. Nanotubes of dititanates are formed by rolling the (100) planes around the [010] axes [32,33]. The layered structure with interlayer distance of $6-8 \AA$ (depending on the temperature of post annealing and thus degree of dehydration) in both monoclinic and orthorhombic structures renders the protons, alkali and alkali earth cations mobile (ionic conductivity in electric field) and easy to replace with other cations (e.g. by ion exchange or intercalation) [1].

Upon calcination at moderate temperatures up to $500{ }^{\circ} \mathrm{C}$, both monoclinic and orthorhombic layered titanates easily transform into the metastable monoclinic $\mathrm{TiO}_{2}-\mathrm{B}$ phase having edge and corner-sharing $\mathrm{TiO}_{6}$ octahedra [34]. The structure of $\mathrm{TiO}_{2}$-B includes nanoscopic channels, which allow advanced ionic transport in these materials as well. Annealing for a longer period of time and/or at higher temperatures result in the formation of bulky $\mathrm{TiO}_{2}$ anatase (up to $700{ }^{\circ} \mathrm{C}$ ) and rutile (over $700{ }^{\circ} \mathrm{C}$ ) phases [35]. Although X-ray diffraction is often used in the literature to reveal the phase composition of the product, due to the very similar diffraction patterns of anatase and $\mathrm{TiO}_{2}-\mathrm{B}$ which show only minor differences in the low angle scattering range, XRD may sometimes be unreliable for analysis. As a complementary method, micro-Raman spectroscopy offers a more accurate tool because of the good separation of various low energy vibration modes in the 80 $220 \mathrm{~cm}^{-1}$ wavenumber window for anatase and $\mathrm{TiO}_{2}-\mathrm{B}$. The intensity ratio of the peaks assigned to the two polytypes can be used to determine the ratio of the two phases in the specimen with good accuracy [36] (Fig. 2).

The microstructure of 1D titanates can be either nanotubular or nanofibrous. Nanotubes may have two forms: (i) nested cylinders in which the nanotubes are in a coaxial arrangement and (ii) rolled-up sheets with spiral shape cross-section. The presence of $2 \mathrm{D}$ titanate nanosheets is necessary to form $1 \mathrm{D}$ structures. Nanosheets, either single or multiple-layers, first form in alkaline hydrothermal conditions from the titania starting material. Asymmetric forces acting on these nanosheets caused by different chemical environments on the opposing sides result in the bending of the structures. In the case of single layers, asymmetric forces do not arise in dispersions, thus such structures remain unfolded according

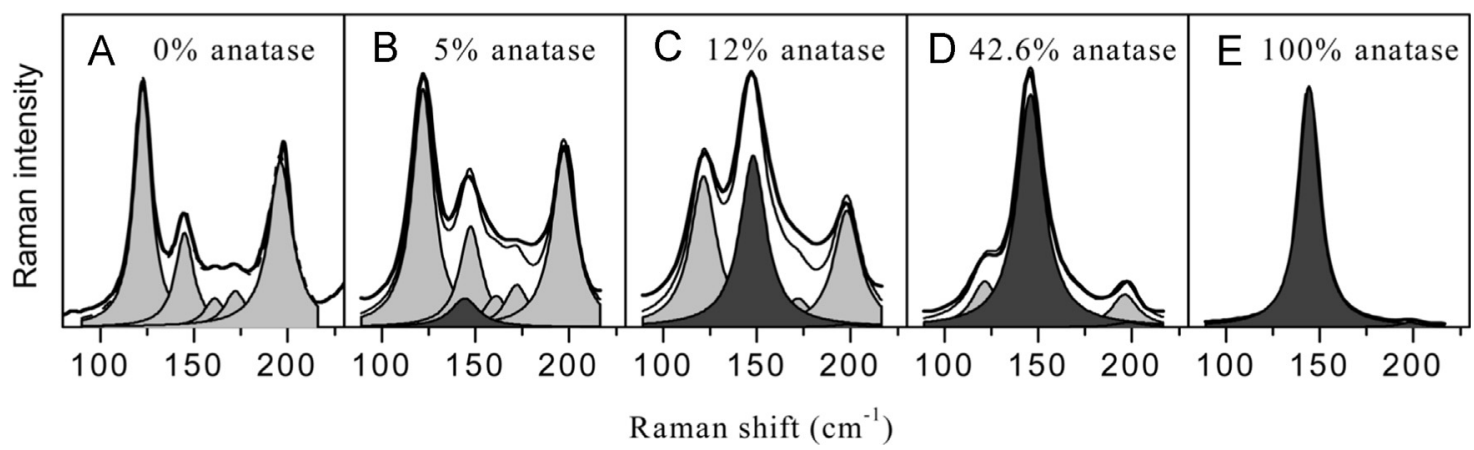

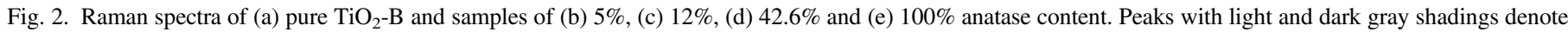
$\mathrm{TiO}_{2}-\mathrm{B}$ and anatase vibration modes, respectively. Reproduced from Ref. [36]. 
to the experience. In contrast to single layers, multi-layered structures experience the alkaline solution on one side and the crystal on the other, which may lead to bending and even exfoliation of the outer layer. According to simulations [37], curved multilayer structures minimize their total energy. For highly curved surfaces, further energy minimization is reached by fusing the opposing edges of the structure leading to various $1 \mathrm{D}$ tubular nanoobjects that can be described by their cross-section such as onions, nested tubes and spirals. By tuning the reaction conditions, a number of different scenarios for crystal growth may be achieved. For instance, when the crystal growth is fast enough, the nanosheets quickly become too robust to withstand the bending forces thus nanofibers form instead of nanotubes [32]. On the other hand, nanowires can also form self-assembled nanotubes by merging nanotube bundles [38]. It is interesting to note, that the process may be reversed by exposing the nanowires to high-energy ball mill, where titanate nanosheets delaminate and then scroll up to nanotubes [31].

\subsection{Physical and chemical properties of titanate nanomaterials}

Titanate nanomaterials such as nanotubes, nanofibers and nanosheets are ionic hydrophilic compounds with high specific surface area up to $\sim 400 \mathrm{~m}^{2} \mathrm{~g}^{-1}$ for nanotubes and nanosheets, and typically between $\sim 20$ and $130 \mathrm{~m}^{2} \mathrm{~g}^{-1}$ for the more robust nanofibers. In certain cases the band gap of nanostructured layered titanates is somewhat higher than those of corresponding titania phases $(\sim 3.8 \mathrm{eV}$ vs. $\sim 3.2 \mathrm{eV})$ due to lower dimensionality, i.e., a 3-D to 2-D transition. Because of the mobile alkali cations (and protons in acid washed powders) the electrical conductivity is significantly better than that of titania nanomaterials, however shows strong dependence on the temperature and humidity of the environment. Elevated temperatures increase the concentration of charge carriers (just like in other semiconductors and ionic conductors) contributing to even higher conductivity but because of the loss of surface water and even recrystallization to dehydrated crystal forms the overall conductivity decreases above $200{ }^{\circ} \mathrm{C}$ [33].

The elastic modulus ( $\sim 30 \mathrm{GPa})[39]$ of titanate nanowires is not outstanding compared to some common materials, however, the reported yield strength of $\sim 3 \mathrm{GPa}[40]$ is rather high, which in combination of with the high aspect ratio (up to $\left.10^{2}\right)$ and relatively low mass density $\left(\sim 3 \mathrm{~g}^{1} \mathrm{~cm}^{-3}\right)$ suggests these materials to be used as additives for mechanical reinforcement of e.g. polymers in composites (Table 1) [41].

While the alkali and alkali earth layered titanates are bases, their protonated forms are solid acids displaying both Bronsted and Lewis acid behavior [63-66]. Determination of their pKa is typically done by titration [65], thermogravimetry or programmed adsorption/desorption (methylene blue [67], pyridine [68], $\mathrm{CO}_{2}$ [69]).

The mobile cations on the surface as well as between the titanate layers make these materials excellent ion-exchange media, which may be directly exploited in e.g. heavy metal ion hazard mitigation in natural waters but also in anchoring various moieties on the surface. Nanocomposites of silver and titanate nanofibers obtained by adsorption and ion exchange of $\mathrm{Ag}^{+}$on hydrothermally grown sodium titanate were demonstrated as antimicrobial coatings [70], whereas adsorbed $\mathrm{Cd}^{2+}$ ions could be converted into $\mathrm{CdS}$ quantum dots [71,72] for photocatalytic applications. Adsorption of $\mathrm{Mg}^{2+}$ followed by the precipitation of stearate ions on the surface lead to a control over its wetting properties [73], but also cationic surfactants such as cetyl-trimethylammonium bromide [74] and poly (diallyldimethylammonium) chloride [75] may be also used for the hydrophobization of titanates.

The $-\mathrm{OH}$ groups being present on the surface of titanates enable covalent functionalization e.g. by the controlled hydrolysis of trialkoxysilanes (e.g. $\left.\mathrm{Si}(\mathrm{EtO})_{3} \mathrm{R}\right)$ in anhydrous solvents to link any organic group via a strong $\mathrm{Ti}-\mathrm{O}-\mathrm{Si}-\mathrm{C}$ bond [76] or by esterification with carboxylic acids in anhydrous alcohol [77]. The linked organic groups may then serve as a template for subsequent chemical reactions, e.g. polymer grafting for nanocomposite applications [78-80].

\section{Surface and near surface analysis of protonated nanowires and nanotubes}

The electronic structure of titanate nanosystem has been studied using a variety of different experimental techniques. Titanate nanotubes (TiONT) and nanowires (TiONW) were synthesized a simple alkali hydrothermal method detailed earlier. In this section we characterize mainly the H-form titanates. The as-prepared titanate nanostructures were washed

Table 1

An overview of titanate nanomaterial properties (Adopted from Ref. [33]).

\begin{tabular}{|c|c|c|c|}
\hline & Nanotube & Nanowire & Nanosheet \\
\hline Diameter or thickness & $8-15 \mathrm{~nm}[9,32,42]$ & $5-300 \mathrm{~nm}[43-45]$ & $<10 \mathrm{~nm}[32]$ \\
\hline Length or lateral dimensions & $\begin{array}{l}\text { Up to several } \\
\text { micrometers [32] }\end{array}$ & $\begin{array}{l}\text { Up to several } \\
\text { micrometers }[43,44]\end{array}$ & $>100 \mathrm{~nm}[32]$ \\
\hline Band gap & $3.1-3.9 \mathrm{eV}[46-49]$ & $3.1-3.6 \mathrm{eV}[48,50]$ & $3.8 \mathrm{eV}[4,46]$ \\
\hline Specific surface area & $\begin{array}{l}50-400 \mathrm{~m}^{2} \mathrm{~g}^{-1} \\
{[9,42,51-53,173]}\end{array}$ & $\begin{array}{l}18-130 \mathrm{~m}^{2} \mathrm{~g}^{-1} \\
{[54-56]}\end{array}$ & $240-380 \mathrm{~m}^{2} \mathrm{~g}^{-1}[57,58]$ \\
\hline $\begin{array}{l}\text { Young's modulus } \\
\text { Electrical conductivity }\end{array}$ & $\begin{array}{l}\text { n.a. } \\
1.5 \times 10^{-6}-7.9 \times 10^{-7} \mathrm{~S} \mathrm{~cm}^{-1}[59,60]\end{array}$ & $\begin{array}{l}\text { 14-46 GPa }[39,40] \\
\sim 10^{-7} \mathrm{~S} \mathrm{~cm}^{-1} \text { for porous } \\
\text { films of } \mathrm{NWs} \text { and } \sim 10^{-1} \mathrm{~S} \mathrm{~cm}^{-1} \\
\text { for individual belts }[61]\end{array}$ & $\begin{array}{l}\text { n.a. } \\
10^{-10} \mathrm{~S} \mathrm{~cm}^{-1} \text { for films } \\
\text { of nanosheets [62] }\end{array}$ \\
\hline
\end{tabular}


thoroughly with deionized water to neutral $\mathrm{pH}$, then subsequently with $0.1 \mathrm{M} \mathrm{HCl}$ solution several times and finally with water again to obtain a neutral salt-free product. In certain cases titanate nanocomposites obtained by other preparation methods and different types of titania are also discussed for comparison. As the electronic structure of titanate nanocomposites cannot be separated from their morphology, in certain cases electron microscopy (HRTEM, SEM), scanning tunneling microscopy (STM) and X-ray diffraction (XRD) studies are discussed.

\subsection{Surface conducting mechanisms}

Despite the tremendous amount of experimental data accumulated in the past century, the unambiguous microscopic description of electronic processes taking place over adsorbent surfaces is still lacking. Conductivity changes during adsorption are caused by changes in the concentration or mobility of charge carriers. Some authors have identified changes in the ion concentration [81-83] as the main physical reason behind conductivity variations, whereas others argued in favor of the principal role of ion mobility [84-88]. Actually, several conduction channels can co-exist on one adsorbent. On the one hand, perfect insulators do not exist because the material always exhibits nonzero bulk conductivity. Charge carriers can be electrons or holes (in semiconductors), ions (in ionic crystals [89] and superionic conductors [90]) and mixed ionic-electronic conduction (MIEC) can also occur [91]. On the other hand, many systems feature mobile surface ions as well, for example protons or ions from the ionic crystal itself (e.g. $\mathrm{Na}^{+}, \mathrm{K}^{+}$) [92]. The former can occur because of dissociation, sample pretreatment or stoichiometry, the latter are frequently incorporated into the structure during alkaline synthesis. The role of protons in conduction was proved by using a proton-injecting palladium electrode $[86,93,94]$ and hydrogen-deuterium exchange studies [95]. The role of other counterions was demonstrated in $\mathrm{NaCl}$-doped proteins: after periods of long conductance it was possible to identify $\mathrm{Na}^{+}$ and $\mathrm{Cl}^{-}$accummulation by energy dispersive $\mathrm{X}$-ray spectroscopy (EDS) on the sample surface in contact with the electrodes [84]. Several mechanisms were suggested to describe conductivity changes and charge carrier generation and migration due to adsorbates.

The conduction mechanism in electron or hole conductor relative humidity sensors (e.g. electroceramics, carbon nanotubes) is well established [96-99]. These adsorbents are not covered by hydroxyl groups that could anchor different water layers for the actual sensing process. Rather, they usually work at high temperature $\left(T \gg 100{ }^{\circ} \mathrm{C}\right)$ and the key step in the sensing process is the charge transfer between the adsorbent and the adsorbate [100-102]. This is often facilitated by defects (e.g. oxygen vacancies) in the crystal. Low temperature $\left(T<100^{\circ}\right.$ C) humidity sensing phenomena follow a different mechanism mainly because of the presence of the adsorbate layer on the surface.

In case of water adsorption one major school of thought is to assume that the dissociation of adsorbates and/or surface groups is increased by orders of magnitude by the presence of the surface. This theory was first published by Fripiat [103] and later adapted and developed by others [81,82,104-106]. Protons and hydroxy ions originating from surface $\mathrm{OH}$ groups and/or the autodissociation of adsorbed water molecules are assumed to be the main charge carriers. Anderson derived the following equation to quantify conductivity in this framework [82]:

$\sigma_{d c}=q \mu \sqrt{(-O H)} \cdot \exp \left(\frac{-q^{2}}{2 \varepsilon r R T}\right) \cdot \exp \left(\frac{-U}{2 R T}\right)$,

where $\sigma_{d c}$ is the conductivity, $(-\mathrm{OH})$ is the surface concentration of hydroxyl groups, $\varepsilon$ is the relative permittivity of the adsorbent, $\mu$ is the ion mobility, $q$ is the charge of the migrating ion, $T$ is the temperature, $R$ is the universal gas constant, $r$ is the equilibrium distance between the charges and $U$ is the activation energy. However, the qualitative [107] and quantitative [81] relationship between permittivity and conductivity was found by independent research groups even before this theory. These results indicate that conductivity increases due to the increase in permittivity during the adsorption process.

Another approach to describe surface conductivity focuses on the migration of dissociated protons between surface groups and adsorbates capable of forming H-bonds (hopping proton transport). However, the underlying theoretical framework was originally developed to describe the phonon-assisted tunneling hopping of electrons, therefore, its application to ionic conductivity raises as many questions as it answers [108,109].

In his famous 1941 Nature paper Albert Szent-Györgyi suggested that a band structure similar to that found in solids could be present in biological macromolecules as well [110]. This idea initiated research on the band structure and semiconducivity of organic molecules [111-115], which resulted in multiple attempts to apply semiconductor theory to describe the adsorption-induced conductivity changes of biopolymers [116-121]. Recently, near room temperature hopping conduction of ionic charge carriers and holes was suggested in onedimensional $\mathrm{TiO}_{x}[122]$ and $\mathrm{Mo}_{6} \mathrm{~S}_{y} \mathrm{I}_{z}[123,124]$ nanostructures and certain biopolymers $[125,126]$, which was gradually replaced by fluctuation induced tunneling with decreasing temperature [127]. Yet another theory explored the effect of high local electric fields on lowering the binding energy of ions present in the adsorbate layer. Field localization is thought to occur on the interface between the high relative permittivity adsorbate layer (e.g. water) and air as depicted in Fig. 3.

This phenomenon was considered for both one dimensional [128] (nanotubes, nanowires, proteins, DNA [130] etc.) and two dimensional [129] (planar surfaces) geometries, and the effect of water adsorption was explained by the corresponding increase in conductivity.

\subsubsection{Dependence of charge transport processes on the amount of adsorbed water}

Titanate nanostructures contain several different types of water (Fig. 4). Systematic magic angle spinning H-NMR studies identified different proton types in the nanotube walls 


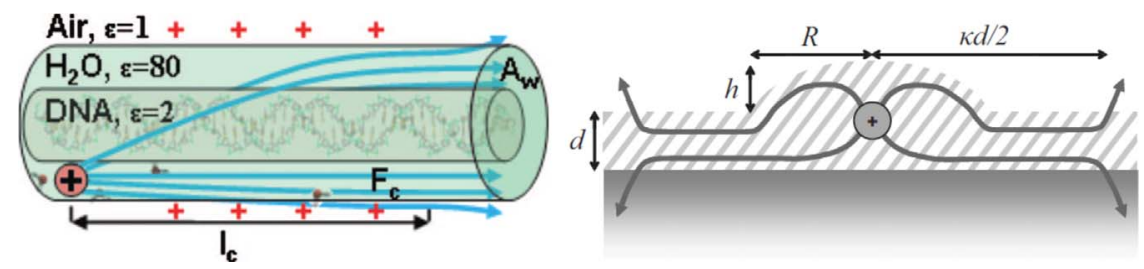

Fig. 3. Electric field localized by a high permittivity adsorbent in the case of (a) DNA (1D) [128] and (b) flat adsorbent surface (2D) [129]. This effect decreases the binding energy of ions in the adsorbate layer.

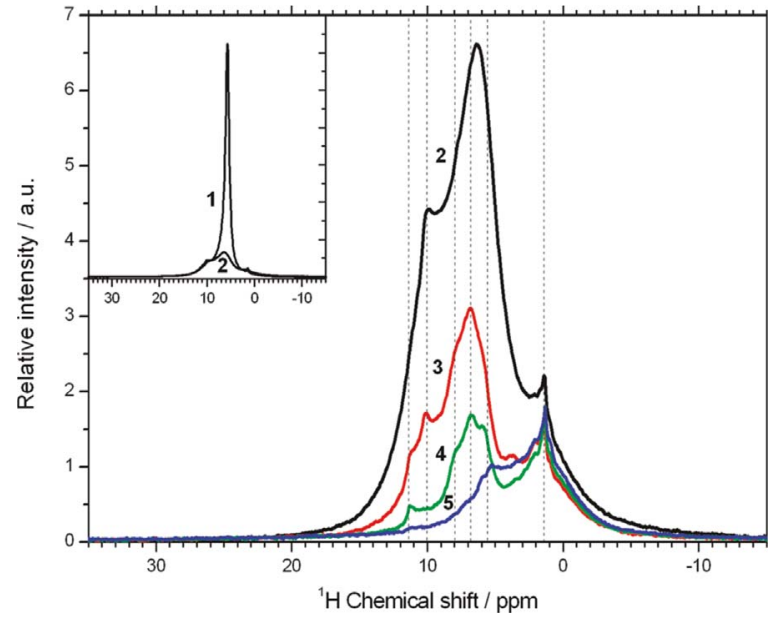

Fig. 4. ${ }^{1} \mathrm{H}$ MAS NMR spectra of H-TiNT annealed at (2) $140{ }^{\circ} \mathrm{C}$, (3) $250{ }^{\circ} \mathrm{C}$, (4) $350{ }^{\circ} \mathrm{C}$, and (5) $450{ }^{\circ} \mathrm{C}$ for $24 \mathrm{~h}$. The inset shows the spectrumof H-TiNT saturated with water vapor of relative humidity $30 \%$ at $25^{\circ} \mathrm{C}$ followed by drying in pure nitrogen for $3 \mathrm{~h}$ (1). Several peaks in the chemical shift region near $1.4 \mathrm{ppm}$ are artifacts, i.e., signals from the NMR rotor. Reproduced from Ref. [131].

including crystallographic water molecules and ionexchangeable $\mathrm{OH}$ groups [131].

Because of the essentially surface nature of adsorptioninduced dielectric processes, the amount of the adsorbed species appears to be the governing physical property of the system. In the case of titanate nanowires it is sufficient to consider only physisorbed water layers in the 6-100 RH\% range [92], therefore, it is feasible to interpret experimental results as functions of adsorbed water amount and surface coverage. Fig. 5. depicts the logarithm of the conductivity of titanate nanowires as a function of adsorbed water amount determined by dielectic spectroscopy.

The characteristics is linear for almost four orders of magnitude, indicating an exponential conductivity dependence in the small surface coverage range (see inset). Therefore, water layers attached to the hydoxylated surface increase conductivity considerably [93], even though TPD studies performed on $\mathrm{SnO}_{2}$ indicated otherwise, i.e. they emphasized the importance of the first chemisorbed layer (surface $\mathrm{OH}$ groups) in determining the conductivity [132]. The exponential increase becomes gentler upon the onset of water condensation, indicating that the formation of bulk-like layers affects conductivity to a smaller extent only.

Ion mobility increases monotonically with water adsorption and the concentration of charge carriers governs conductivity changes [92]. Surface induced dissociation suggested by

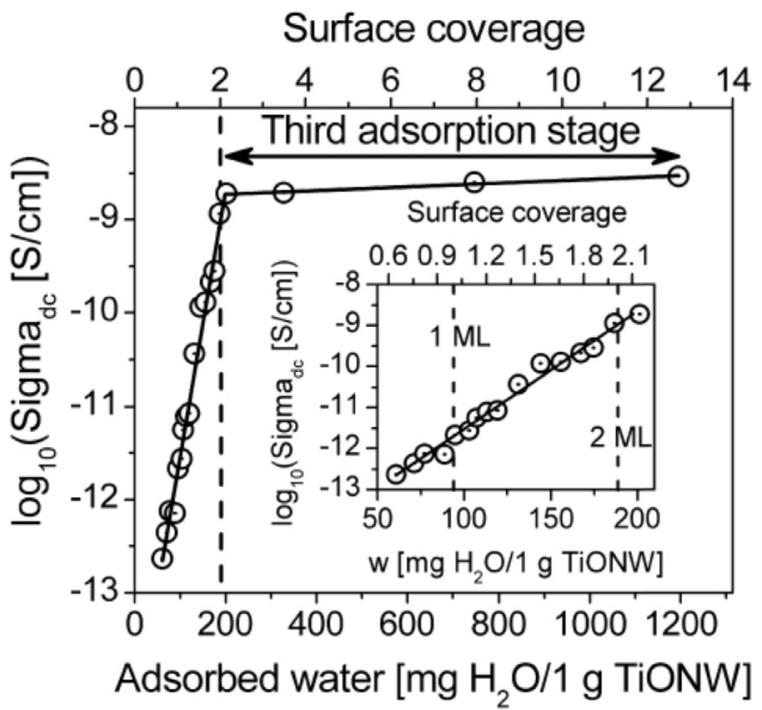

Fig. 5. Logarithm of the conductivity of titanate nanowires as a function of adsorbed water amount. Surface coverage calculated from the GAB model is also depicted. Lines in the figure are results of linear fits to the data. Reproduced from Ref. [92].

Fripiat [103] can be described by the following equation:

$\sigma_{d c}=n \frac{\nu(e z)^{2} a^{2}}{k T} \exp \left(\frac{l(e z)^{2} n}{k T A \rho}\right)$,

where $\sigma_{d c}$ is the conductivity, $\nu$ is the frequency of the charge jumping attempt, $e$ is the elementary electric charge, $z$ is the valency of the ion, $a$ is the distance between the two equilibrium positions $(\sim 8 \AA), l$ is the distance from the surface, $k$ is Boltzmann's constant, $T$ is the temperature, $A$ is the specific surface area of the sample and $\rho$ is its density. The model assumes that all charge carriers originate from the dissociation of water molecules hitting the surface, therefore, their concentration is proportional with the number of water molecules:

$n=\alpha \cdot N_{\text {water }}$,

where $N_{\text {water }}$ is the number of water molecules hitting the surface in $1 \mathrm{~cm}^{3}$ sample and $\alpha$ is the dissociation ratio. Experimentally determined values for the latter exceeded that found in liquid water by a factor of $10^{6}[103,105]$.

Eq. (3). defines a linear relationship between adsorbed water molecules and generated charge carriers, whereas in titanate nanowires a more pronounced dependence was found experimentally. Fig. 6 depicts the dependence of the logarithm of charge carrier concentration determined by ionic current method [92] on the concentration of adsorbed water molecules. 


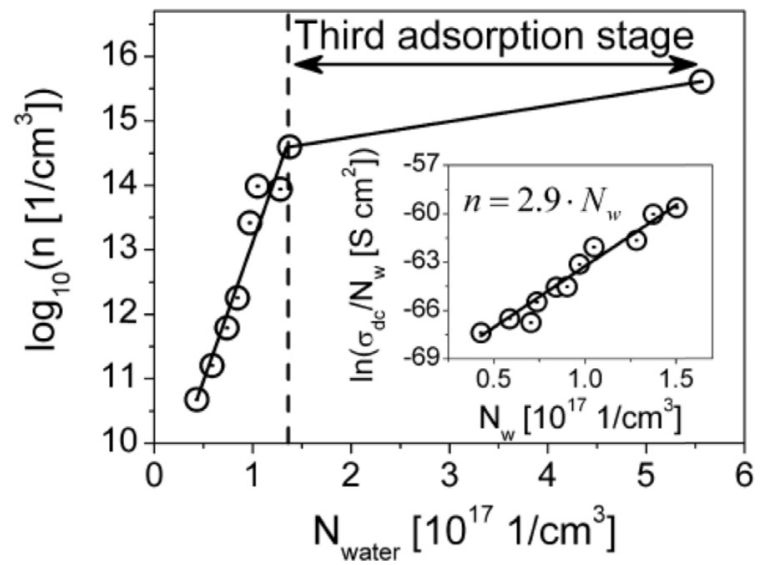

Fig. 6. Effect of increasing the concentration of adsorbed water molecules on the charge carrier concentration in titanate nanowires as determined by the ionic current method. Reproduced from Ref. [92]. The inset depicts the same data in the representation suggested by Clement [105].

In the small adsorbed amount range the dependence in Fig. 6 is exponential like in Fig. 5. Therefore, the original linear dissociation hypothesis cannot be upheld in this case. However, a decade later Clement argued that a linear relationship between $\ln \left(\sigma_{d c} / N_{v i z}\right)$ and $N_{v i z}$ confirms the Fripiat model. Such linear relations were found already before Fripiat for the $\mathrm{NH}_{3} / \mathrm{SiO}_{2}$ and $\mathrm{CH}_{3} \mathrm{OH} / \mathrm{SiO}_{2}$ systems [133] but this line of thought was not pursued any further at that time. The inset in Fig. 6 shows data measured on titanate nanowires in the Clement representation. It is interesting to note that even though the validity of Eq. (3) can be refuted on the basis of Fig. 6, the Clement representation is acceptably linear. However, the calculated dissociation ratio is $\alpha=2.9$ which does not have any physical meaning. The root of the issue is in Eq. (2) of the Fripiat model that claims that the conductivity of the adsorbent is an exponential function of the charge carrier concentration. Actually, these two quantities are linearly dependent on each other by definition $\left(\sigma_{d c} \propto n\right)$. The apparently linear relationship in the inset of Fig. 6 is an artifact caused by the superposition of the exponential dependence between ion concentration and adsorbed amount and the linear dependence between conductivity and ion concentration. Therefore, the validity of the Fripiat model has not been confirmed by the Clement represenation in adsorption induced conduction processes.

Let us now consider the Anderson variant of the Fripiat model again (Eq. (1)) [82]. According to the Nernst-Thomson rule a high permittivity solvent decreases the anion-cation attraction [134], and the dissociation energy changes to $1 / \varepsilon$ of its original value [81]. Since water adsorption increases the permittivity of the adsorbent, this should facilitate the dissociation of water molecules hitting the surface. The experimental validation of this hypothesis is based on plotting the logarithm of the conductivity as a function of the inverse permittivity and expecting a linear trend [81,82]. Low frequency dispersive processes (LFD, MWS, EP) in the dielectic spectra of hydrated adsorbents make it difficult to determine static permittivity accurately. Measured values are influenced considerably by the experimental conditions: frequency, voltage and electrode material. Moreover, the Anderson model forecasts an exponentially decreasing conductivity with increasing surface $\mathrm{OH}$ group concentration, whereas the opposite trend was found for the dehydroxilation of kaolinite earlier [135]. Conductivity was found to increase with the dipole moment of the adsorbed species in Vycor-glass [107]. Experimental proof for proton exchange between surface functional groups and adsorbed molecules was obtained in $\mathrm{CH}_{3} \mathrm{OH} / \mathrm{SiO}_{2}$ [136], water/SBA-15 [137] and various water/ sulphated polystyrene [138] systems. The exchange was heavily dependent on surface coverage [135,136] and one adsorbate could be protonated multiple times while being anchored at a specific adsorption site. The importance of functional groups in conductive processes was experimentally confirmed in various amine-intercalated titanium(IV)-phosphate [139] and zirconium hydrogen phosphate [140-142] systems. Moreover, the dissociation of functional groups was identified as the governing step of charge carrier generation in proteins [95], acid functionalized SBA-15 [137] and metalorganic frameworks [143]. Conductivity increased with the increasing $\mathrm{p} K_{a}$ of the surface functional groups and ion concentration was found to be the key parameter determining the conduction. A strong $\mathrm{pH}$-dependence of proton transport related dielectic processes was also revealed in proteins $[86,144]$.

The details of ionic surface diffusion are as unclear as those of the charge carrier generation mechanism despite the large body of experimental data available [145-147]. A cooperative proton transfer mechanism (flip-flop hydrogen bonding, domino mechanism) [148] was found in continuous water clusters adsorbed on proteins by neutron diffraction analysis. This phenomenon is likely to be similar to the cooperative polarization mechanism found earlier in silica gel [149]. The subsequent breakage and reformation of hydrogen bonds was assumed to be responsible for the conduction of ice as early as the 1950s [150]. More recent results suggest that adsorbed molecules at the $\mathrm{S} / \mathrm{G}$ interface can actively participate in Grotthuss-type proton conduction [151]. Several variants of this process were published: the one occuring in hydrated poly (vinyl-phosphonic acid) polymers is denoted as carrier mediated [152], while the one found in hydrated boehmite is called interfacial Grotthuss mechanism [153].

\subsubsection{Dependence of the activation energy on water adsorption}

It is well-known that the activation energy of dielectric processes (relaxation as well as conduction) changes with the water content/surface coverage of hydrated hydrophilic materials. However, several different trends were described already. The activation energy of conduction decreases with increasing surface coverage in certain oxides $[107,133,163]$, clay materials [163,164], zeolites [154,160,163], layered zirconium phosphonates [140], amine-intercalated titanium(IV)-phosphate [139] and proteins [117,161]. It increases (or stays almost constant) in collagene [159] and in zeolite [169] and silica/ alumina catalyst systems [155], and it changes as a maximum function in certain textiles [116] and Vycor-glass [172]. The 
activation energies of various relaxation processes decreased with increasing surface coverage in clay materials [162] and polymer composites [165] but increased in $\mathrm{Fe}_{2} \mathrm{O}_{3}$ [156], $\mathrm{Ag}_{2} \mathrm{O}$ [168], silica [170,176], calcium-silicate-hydrate $[157,158]$ and ovalbumine adsorbents [166,171].

The conductivity of titanate nanostructures is an exponential function of both temperature and adsorbed water amount. Such parallel exponential dependence can only occur if the activation energies of the individual processes are linear functions of the adsorbed water amount. Such linear dependence was actually found experimentally in ionic conductor polymers [88]:

$\sigma_{d c} \propto \frac{1}{T} e^{\left(-\frac{\left(E_{a}-b^{*} w\right)}{k_{B} T}\right)}$,

where $b^{*}=b k_{B} T$ is a temperature-dependent exponent, $b$ is a fitting parameter, $T$ is the temperature, $k$ is Boltzmann's constant, $w$ is the amount of adsorbed water and $E_{a}$ is the activation energy. It is typical to consider only the possibility of decreasing activation energy since the decreasing potential barrier resulting from water adsorption should result in higher conductivity. Therefore, the opposite effect is to be expected if activation energy increases with water adsorption. On the other hand, substituting the exponential conductivity vs. water contents expression into the Arrhenius equation results in

$$
\begin{aligned}
\ln \sigma_{d c} & =(A+B \cdot w)-\frac{(C \pm D \cdot w)}{k_{B} T} \\
& =\left(A-\frac{C}{k_{B} T}\right)+\left(B \mp \frac{D}{k_{B} T}\right) w,
\end{aligned}
$$

where $(A+B w)=\ln \sigma_{0},(C+D w)=E_{a}$ is the activation energy at adsorbed water amount $w$, while $A$ and $C$ are the preexponential constant and the activation energy of the hypothetical dry state $(w=0)$. The $\left(A-C /\left(k_{B} T\right)\right)$ part equals the Arrhenius-type temperature dependent $\ln \sigma_{d c}$ for a dry adsorbent, and the second term is the slope of the conductivity characteristics. Arrhenius-type temperature dependence is found indeed for all water amounts, whereas the $1 / T$ type behavior of the second term in the right hand side has been verified earlier in hydrated collagene [159]. Since increasing the surface coverage can results in both decreasing $[107,117,133,140,160-165]$ and increasing [159,166-171] activation energies, non-monotonous trends [116,172] are likely to be due to the simultaneous occurence of the different processes. Earlier it was assumed that the dynamics is governed by charge carrier generation and transfer processes [107]. However, it is not possible to identify the individual effects of such closely related processes by dielectric spectroscopy alone.

Fig. 7 depicts the activation energy (a) and preexponential constant (b) of the conductance of titanate nanowires as functions of adsorbed water amount determined from experimental data collected above $0{ }^{\circ} \mathrm{C}$ [173]. The linear trends described by Eq. (5) and observed earlier in hydrated collagene are clearly present for both parameters.

Correlation between Arrhenius-parameters is a well-known phenomenon. A linear relationship between the activation

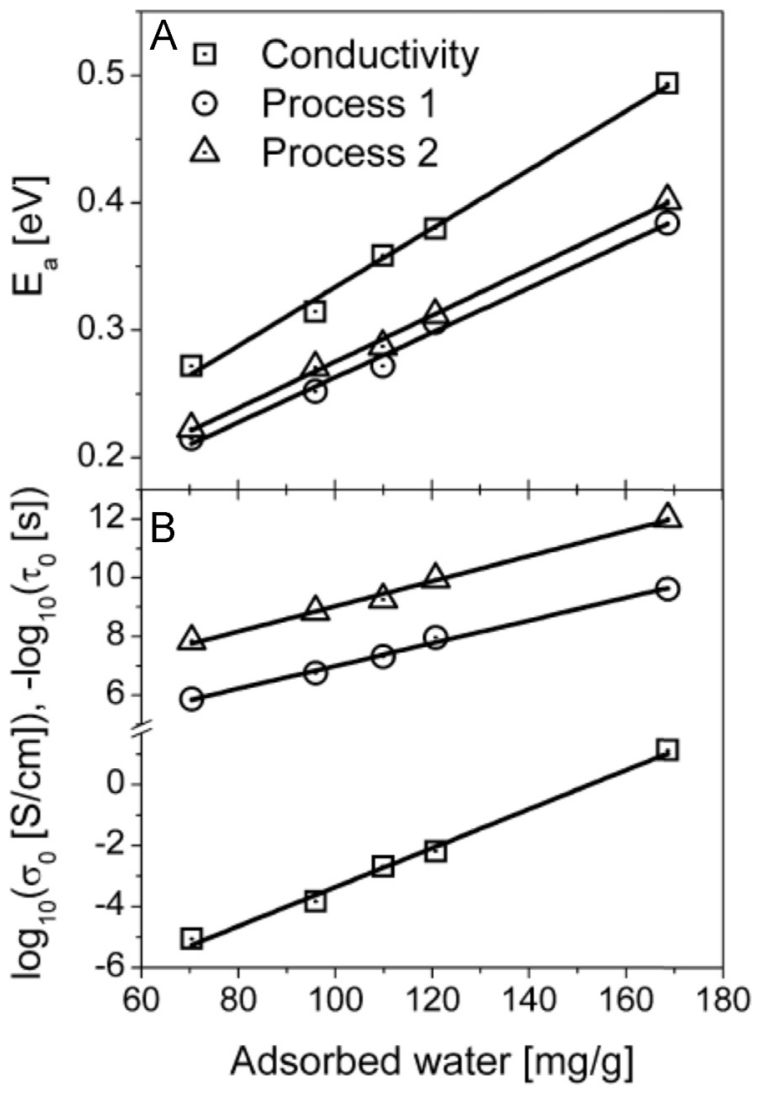

Fig. 7. Activation energy (a) and preexponential constant (b) of the conductance of titanate nanowires as functions of adsorbed water amount. Reproduced from Ref. [173].

energy and the preexponential constant was found in a host of diverse physico-chemical processes including catalysis [174], conductance [161,175] and dielectric relaxation [176,177]:

$\log _{10} \sigma_{0}=a E_{a}+b$

where $a$ and $b$ are characteristic constants of the system studied and $E_{a}$ is the activation energy. This is the so-called compensational effect, also known as isokinetic relationship, enthalpy-entropy compensation, Barclay-Butler-rule, thetarule or Smith-Toppley effect [178]. In conductive processes the effect is known as the Meyer-Neldel rule [179,180]. Fig. 8 depicts the correlation between the Arrhenius parameters of the titanate nanowire system. This finding confirms the validity of the Meyer-Neldel rule in the case of one dimensional layered titanate nanomaterials.

\subsection{X-ray photoelectron spectroscopy (XPS)}

The electronic structure and surface composition of $\mathrm{H}$-form titanate nanowires and nanotubes was studied by X-ray photoelectron spectroscopy. The $\mathrm{Ti} \quad 2 \mathrm{p}_{3 / 2}$ maximum $(458.9 \mathrm{eV})$ was used as binding energy reference. The same data were obtained when $\mathrm{C} 1 \mathrm{~s}$ (adventitious carbon at $285.1 \mathrm{eV})$, or $\mathrm{O} 1 \mathrm{~s}$ lattice oxygen $(530.4 \mathrm{eV})$ was used as reference. Sample preparation (oxidation-reduction) for XPS measurements was carried out in situ in preparation chamber. 


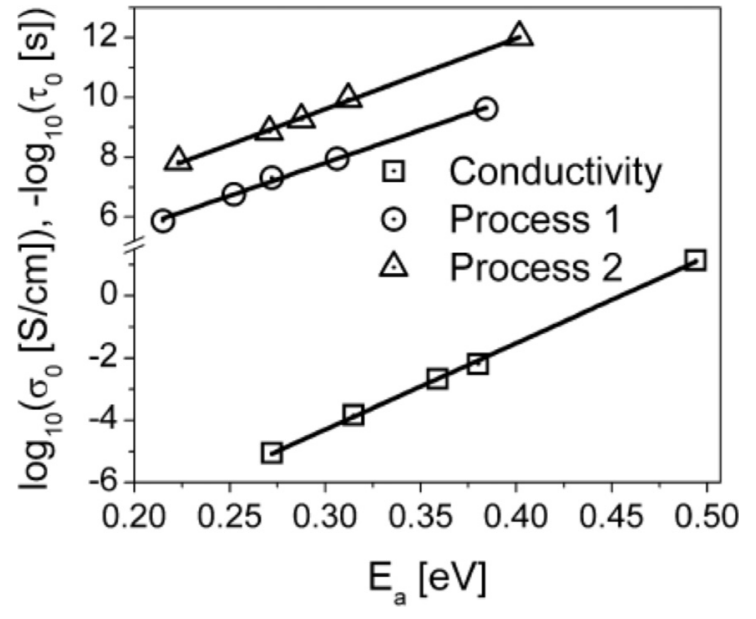

Fig. 8. Correlation between the activation energy and the preexponential constant of the conductance. Lines connecting the points are results of linear fits and they indicate that the compensation effect is at work here. Reproduced from Ref. [173].

The sample preparation chamber was directly connected to the measuring chamber to avoid the contamination of samples between the steps. Self-supporting pellets were used in XPS measurements. The impurity level of nanocomposites was below $1 \%$. The foreign elements identified by XPS were C, $\mathrm{Ca}$, and $\mathrm{Na}$ that remained in the sample from the preparation process $[52,181-183]$.

Fig. 9(A) and (B) shows the photoemissions measured on protonated nanowires and nanotubes kept at different temperatures for $60 \mathrm{~min}$ and cooled down to $300 \mathrm{~K}$. The doublet spectral line of $\mathrm{Ti} 2 \mathrm{p}$ for the titanate nanostructures is characterized by a binding energy of $458.9 \mathrm{eV}\left(2 \mathrm{p}_{3 / 2}\right)$ and $464.5 \mathrm{eV}\left(2 \mathrm{p}_{1 / 2}\right)$ with separation energy of $5.6 \mathrm{eV}$. Spectral lines of both the Ti $2 p_{3 / 2}$ and $\operatorname{Ti} 2 p_{1 / 2}$ are very close to the reported values obtained earlier on several $\mathrm{TiO}_{2}$ samples. Similar symmetric Ti $2 p$ feature with same energy separation was observed when amorphous precursor $\left(\mathrm{TiCl}_{3}\right)$ was used for preparation [184]. Note, however, that the surface defects can cause band bending and a rigid shift of the whole spectrum. This probably accounts for the scatter in experimental peak positions [2,3,52,185-188]. Only one symmetric signal was measured for $\mathrm{Ti} 2 \mathrm{p}_{3 / 2}$ up to $800 \mathrm{~K}$ for nanowires and nanotubes [183]. The observed photoemission for $\mathrm{O} 1 \mathrm{~s}$ $(530.4 \mathrm{eV})$ is not symmetric. The analysis of thermal gravimetric (TG) and ion flow curves (DTG-MS) give general picture about the reactions during heat treatment and help in interpretation of XPS data [183].

DTG-MS results the peak corresponding to water decreased sharply between 293 and $573 \mathrm{~K}$. The MS intensity of the peak for adsorbed water decreased significantly between 350 and $650 \mathrm{~K}$ (Fig. 10). The sources of water formation are the adsorbed water (or lattice water) [92,194], and the surface reaction of surface $\mathrm{OH}$ groups and hydrogen from the recrystallization process. These reactions could significantly increase the number of defects in nanowires and nanotubes and may promote the phase transformation of titanates. We should mention that a slower thermal release of water moieties from
TiONT compared to TiONW, in harmony with the literature data [92,189], may have its origin in the condensation of water in TiONT channels. Interestingly, around $700 \mathrm{~K}$ the $\mathrm{O} 1 \mathrm{~s}$ photoemission at $533.5 \mathrm{eV}$ reappeared on nanotubes (Fig. 9 (B)). This could be the result of some hydrocarbons formed in the reaction between $\mathrm{OH}$ groups and carbon fragments.

A series of Ti $2 p_{3 / 2}$ and Ti $2 p_{1 / 2}$ signals as a function of pretreated temperatures from nanowires and nanotubes is depicted in Fig. 9(A) and (B). It is remarkable that the shapes of the photoemission peaks ( $\mathrm{Ti} 2 \mathrm{p}_{3 / 2}$ and Ti $2 \mathrm{p}_{1 / 2}$ ) were rather symmetric in spite of the fact that some defects could form during water releases. The reason of the absence of $\mathrm{Ti}^{3+}$ on top of surface region could be the fast oxygen transport from bulk to surface during heating/cooling process. However, the somewhat higher FWHM for Ti $2 \mathrm{p}_{3 / 2}(1.56 \mathrm{eV})$ than it was postulated in literature $(1.50 \mathrm{eV})$ [185] and its slight change with temperature may reflect to the some defects in topmost layer and formation of anatase nanostructures (see Raman discussion below). When the XP measurements were carried out in-situ experiments conditions, an additional weak $\mathrm{Ti}^{3+}$ signal was detected [191]. After subtracting the inelastic background, two doublets were necessary to fit the Ti $2 \mathrm{p}$ signal. The BE value reported in literature for $\mathrm{Ti}^{4+} 2 \mathrm{p}_{3 / 2}$ at $459.5 \mathrm{eV}$. In general this peak shifts to lower binding energy when the valence state of $\mathrm{Ti}^{4+}$ is reduced to $\mathrm{Ti}^{3+}[185,190]$. Then, the peak around 459.1-459.4 correspond to $\mathrm{Ti}^{4+}$, while the small peak used fit the spectra at $\mathrm{BE}$ values of $457.5-458.1$ correspond to reduced $\mathrm{Ti}^{3+}$, suggesting that a small fraction of $\mathrm{Ti}^{4+}$ cations are reduced during annealing to $773 \mathrm{~K}$ under $\mathrm{N}_{2}$ [191]. The reduction degree of these cations with the annealing temperature was followed by treating nanotubes sample in situ in inert atmosphere at different temperatures. When the sample was annealed the population of reduced $\mathrm{Ti}^{3+}$ atoms increased giving a $\mathrm{Ti}^{3+} / \mathrm{Ti}^{4+}$ surface atomic ratio of 0.046 and 0.06 at 573 and $773 \mathrm{~K}$, respectively (Table 2).

Concomitantly, the $\mathrm{O} / \mathrm{Ti}$ surface atomic ratio decreased from 2.48 to 1.89 in the samples treated at $383 \mathrm{~K}$ and $673 \mathrm{~K}$, respectively, suggesting a non-stoichiometric surface composition after annealing. In the other words, surface oxygen deficient $\mathrm{TiO}_{2-x}$ anatase phase are produced by thermally treating. Controversially, after annealing at $773 \mathrm{~K}$ the $\mathrm{O} / \mathrm{Ti}$ surface atomic ratio increased as well the amount of $\mathrm{Ti}^{3+}$, in comparison to the nanotubes thermally treated at $673 \mathrm{~K}$ (see Table 2). These results supported the assumption that Raman shift of the $A_{g 1}$ vibration mode (see below in Raman discussion) is due to partially reduced $\mathrm{Ti}^{3+}$ atoms or oxygen deficient anantase phase $\mathrm{TiO}_{2-x}[191]$.

We may compare the oxygen mobility on titanate nanostructure and well-defined $\mathrm{TiO}_{2}(110)$, too. In both cases the samples were sputtered by $\mathrm{Ar}^{+}$or $\mathrm{Ne}^{+}\left(2 \mathrm{keV}, 1.35 \times 10^{17}\right.$ ions $/ \mathrm{cm}^{2}$ ) at room temperature. The sputtering effect was not detectable on nanostructures after finishing the bombardment and subsequent evacuation of noble gas to reach the UHV condition (not shown). After repeating the experiment with $\mathrm{TiO}_{2}(110)$, the effect was remarkable. 
A
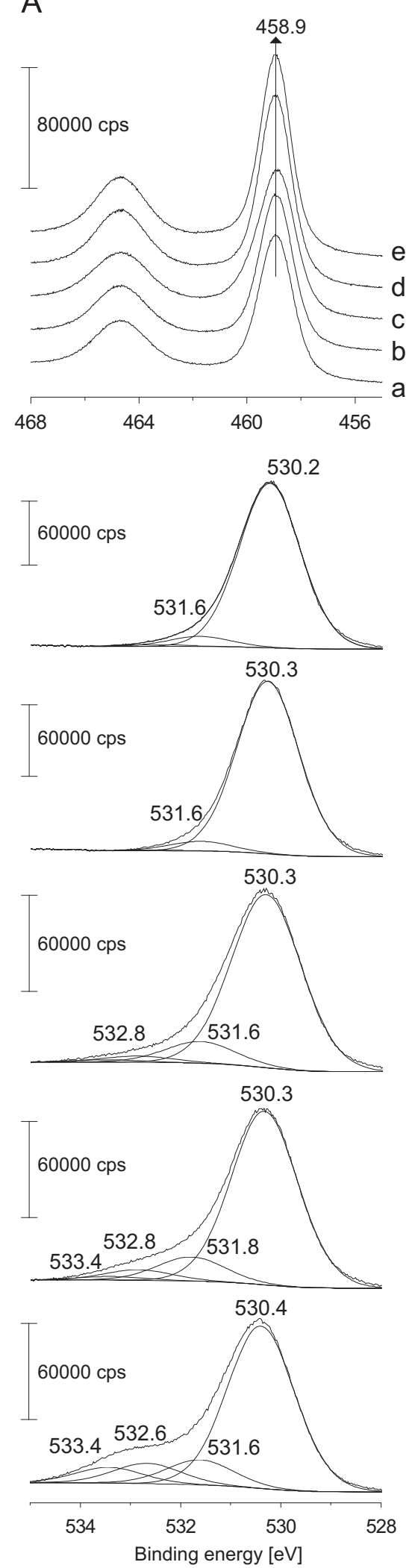

B
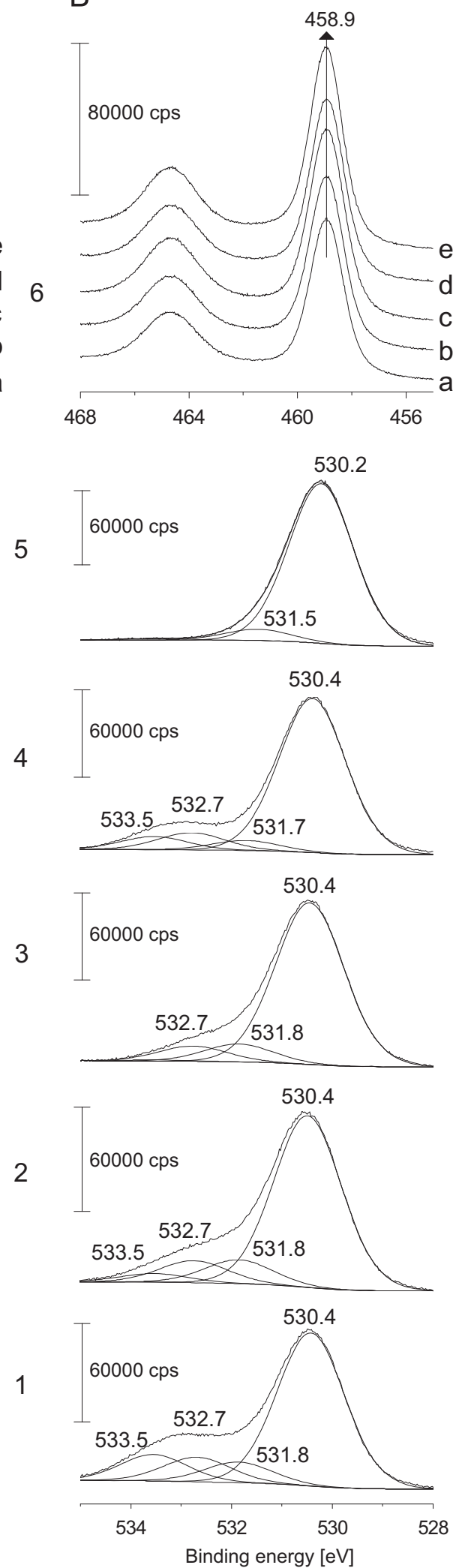

Fig. 9. XP photoemission signals obtained on titania nanowires (NW) (A) and nanotubes (NT) (B). Ti 2p binding energies detected after heat treatment at $298 \mathrm{~K}$ (a), $473 \mathrm{~K}$ (b), $573 \mathrm{~K}$ (c), $673 \mathrm{~K}$ (d), and $773 \mathrm{~K}$ (e). O 1s XP photoemission obtained after heat treatment at $293 \mathrm{~K}(1), 473 \mathrm{~K}(2), 573 \mathrm{~K}(3), 673 \mathrm{~K}(4)$, and $773 \mathrm{~K}(5)$. The spectra were taken at $298 \mathrm{~K}$. Reproduced from Ref. [183]. 
The XP photoelectron spectrum of an annealed, stoichiometric $\mathrm{TiO}_{2}(110)$ is shown in Fig. 11. The Ti $2 \mathrm{p}$ emissions were symmetric; Ti $2 \mathrm{p}_{3 / 2}$ appeared at $458.8 \mathrm{eV}$ corresponding to $\mathrm{Ti}^{4+}$. The noble gas ion sputtering made it possible to reduce the $\mathrm{TiO}_{2}(110)$. The $\mathrm{Ne}^{+}$bombarded $\mathrm{TiO}_{2}(110)$ surface showed significant broadening towards the lower binding energy side, attributed to the transformation of a part of $\mathrm{Ti}^{4+}$ ions to $\mathrm{Ti}^{3+}(457.1 \mathrm{eV})$ and $\mathrm{Ti}^{2+}(455.2 \mathrm{eV})$ $[192,193]$. The spectral feature did not change significantly with time in an inert atmosphere $\left(\mathrm{N}_{2}\right)$. This comparison clearly shows that the mobility of oxygen is much faster on titante nanostructure.

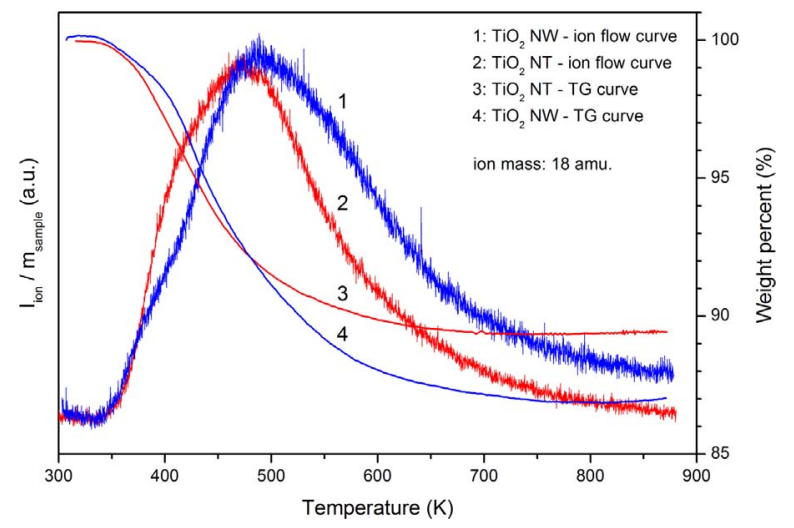

Fig. 10. TG and ion-flow curves of titania nanowires and nanotubes. (1) mass spectrometric intensity at 18 amu recorded during the linear heating of titanate nanowire, (2) MS intensity at $18 \mathrm{amu}$ during the linear heating of titanate nanotubes, (3) thermal gravimetric curve of nanowires, and (4) nanotubes during the linear heating. Heating rate was $10 \mathrm{~K} / \mathrm{s}$. Reproduced from Ref. [183].
The effect of annealing temperature on the structural and bonded states of titanate nanotube films was also studied [194]. Titanate nanotubes were synthesized by a commercial titania $\left(\mathrm{TiO}_{2}\right)$ nanoparticle powder as starting material. A conversion from nanoparticles to nanotubes was achieved by treating the nanoparticles powder with $\mathrm{NaOH}$ at $150{ }^{\circ} \mathrm{C}$, for $48 \mathrm{~h}$, in the autoclave. Only the precipitates were neutralized thoroughly

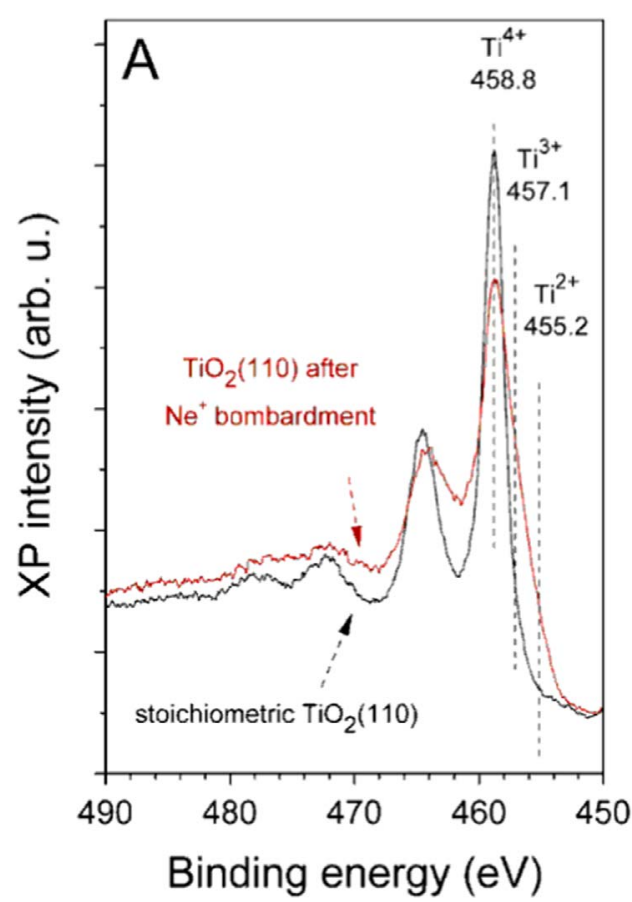

Fig. 11. The $\mathrm{Ti} 2 \mathrm{p}$ region of the nearly stoichiometric $\mathrm{TiO}_{2}(110)$ surface and after $\mathrm{Ne}^{+}$bombardment. Reproduced from Ref. [193].

Table 2

XPS parameters of Ti $2 p_{3 / 2}$ and O 1s derived from spectral fitting. Reproduced from Ref. [191].

\begin{tabular}{|c|c|c|c|c|c|}
\hline Annealing temperature $\left({ }^{\circ} \mathbf{C}\right)$ & Assignment & Binding energy $(e \mathrm{~V})$ & FWHM $^{\mathrm{a}}(\mathbf{e V})$ & Surface atomic ratio $\mathrm{Ti}^{3+} / \mathrm{Ti}^{4+}$ & Surface atomic ratio $\mathrm{O} / \mathrm{Ti}$ \\
\hline 110 & $\begin{array}{l}\mathrm{O} 1 \mathrm{~s} \\
\mathrm{Ti}^{3+} 2 \mathrm{p}_{3 / 2} \\
\mathrm{Ti}^{4+} 2 \mathrm{p}_{3 / 2}\end{array}$ & $\begin{array}{l}530.8 \\
457.5 \\
459.1\end{array}$ & $\begin{array}{l}1.3 \\
1.2 \\
1.2\end{array}$ & 0.026 & 2.48 \\
\hline 200 & $\begin{array}{l}\mathrm{O} 1 \mathrm{~s} \\
\mathrm{Ti}^{3+} 2 \mathrm{p}_{3 / 2} \\
\mathrm{Ti}^{4+} 2 \mathrm{p}_{3 / 2}\end{array}$ & $\begin{array}{l}530.8 \\
457.8 \\
459.2\end{array}$ & $\begin{array}{l}1.2 \\
1.2 \\
1.1\end{array}$ & 0.048 & 2.17 \\
\hline 300 & $\begin{array}{l}\mathrm{O} 1 \mathrm{~s} \\
\mathrm{Ti}^{3+} 2 \mathrm{p}_{3 / 2} \\
\mathrm{Ti}^{4+} 2 \mathrm{p}_{3 / 2}\end{array}$ & $\begin{array}{l}530.8 \\
458.1 \\
459.4\end{array}$ & $\begin{array}{l}1.2 \\
1.4 \\
1.1\end{array}$ & 0.046 & 1.96 \\
\hline 400 & $\begin{array}{l}\mathrm{O} 1 \mathrm{~s} \\
\mathrm{Ti}^{3+} 2 \mathrm{p}_{3 / 2} \\
\mathrm{Ti}^{4+} 2 \mathrm{p}_{3 / 2}\end{array}$ & $\begin{array}{l}530.9 \\
458.1 \\
459.4\end{array}$ & $\begin{array}{l}1.2 \\
1.4 \\
1.1\end{array}$ & 0.045 & 1.89 \\
\hline 500 & $\begin{array}{l}\mathrm{O} 1 \mathrm{~s} \\
\mathrm{Ti}^{3+} 2 \mathrm{p}_{3 / 2} \\
\mathrm{Ti}^{4+} 2 \mathrm{p}_{3 / 2}\end{array}$ & $\begin{array}{l}530.8 \\
458.1 \\
459.4\end{array}$ & $\begin{array}{l}1.2 \\
1.6 \\
1.1\end{array}$ & 0.060 & 1.97 \\
\hline
\end{tabular}

${ }^{\mathrm{a}}$ Full width at half maximum. 

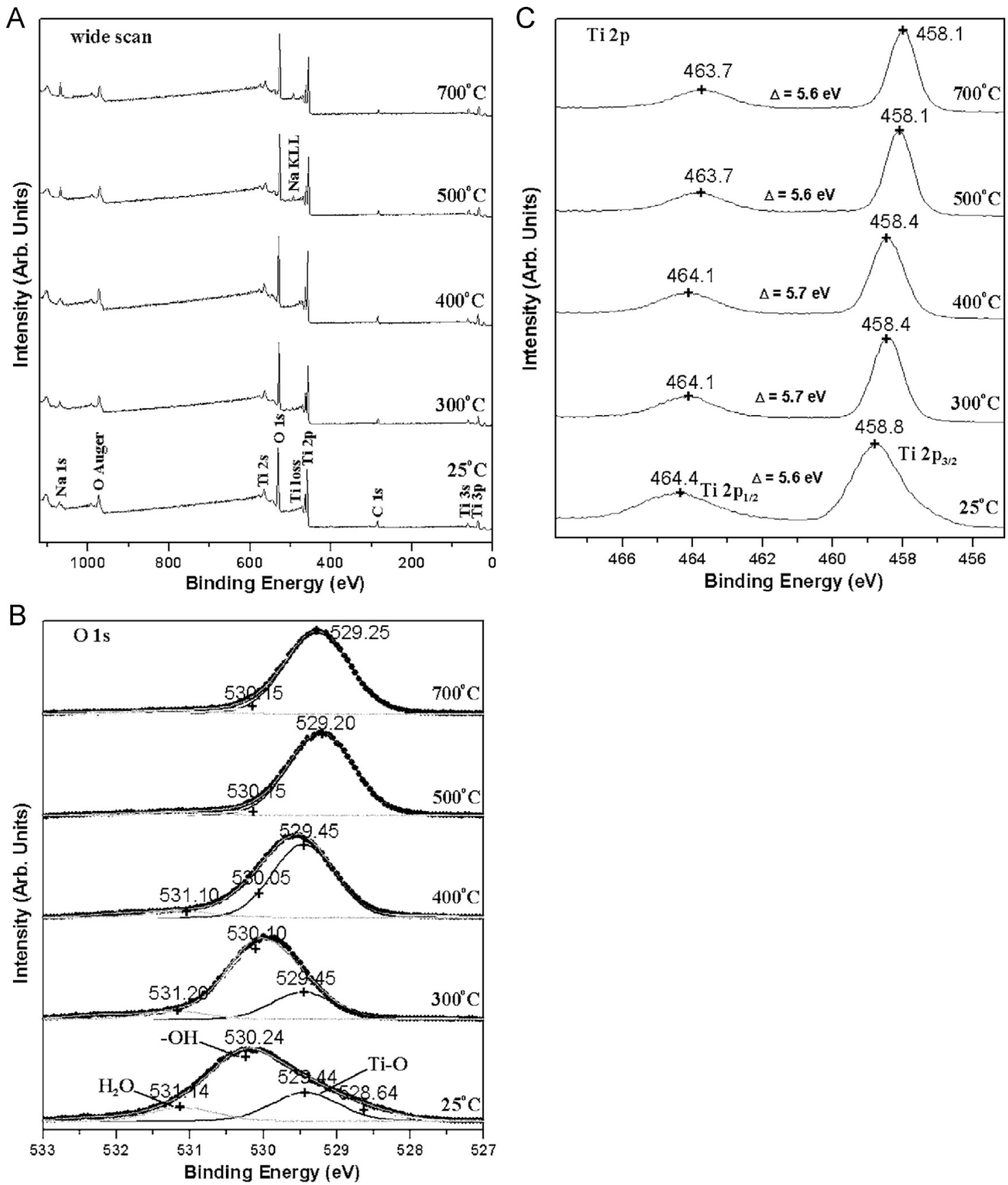

Fig. 12. Photoelectron spectra of the titanate film on the Si substrate as a function of annealing temperature: (a) wide scan survey; (b) O 1s; and (c) Ti 2p. Reproduced from Ref. [194].

with distilled water. Subsequently, titanate films were fabricated on $\mathrm{Si}(001)$ substrates using an electrophoretic deposition (EPD) method. The surface bond states of were analyzed by XPS. The Ti $2 \mathrm{p}_{3 / 2}$ photoemisson peak was symmetric, the peak position was measured at $458.8 \mathrm{eV}$ with FWHMs of $1.82 \mathrm{eV}$. As the annealing temperature increases, the $\mathrm{Ti} 2 \mathrm{p}$ position shifts slightly towards the lower binding energies with a FWHM of $1.17 \mathrm{eV}$ at $673 \mathrm{~K}$. Above $773 \mathrm{~K}$, there was no further shift in peak position $(458.1 \mathrm{eV})$, the FWHM was $1.94 \mathrm{eV}$ (Fig. 12). The peak positions and separation match closely with the reported values for $\mathrm{Ti}^{4+}$ in bulk $\mathrm{TiO}_{2}$ [195]. It was concluded from XPS that during the annealing treatment, the bonded states such as $\mathrm{H}_{2} \mathrm{O}$ and $-\mathrm{OH}$ are removed from titanate films as well as converting the chemical bonded states of titanate to that of titania.

Finally we may conclude that heat treatment induces the reduction of $\mathrm{Ti}^{4+}$ in titanates to $\mathrm{Ti}^{3+}$ and $\mathrm{Ti}^{2+}$, but their detection in the surface layers is not always successful due to the fast oxygen transport from bulk to surface.

The observed photoemission for $\mathrm{O} 1 \mathrm{~s}$ obtained on protonated titanate nanowires and nanotubes is more complex, they are displayed in Fig. 12(A) and (B). Notably, that similar 
complex feature was also observed in the case of in-situ heat treated experiments [191] and on electrodeposited titanate film [194] and when different precursor molecule was used in preparation [184]. Multiple fits were applied on the basis of literature data obtained by angle-resolved XPS experiments. The major contribution arises from the bulk $\mathrm{O}^{2-}$ oxygen atoms, located at $530.4 \mathrm{eV}$, which is the value usually reported for $\mathrm{TiO}_{2}$ samples [183]. The angle-resolved XPS experiments suggest strongly that four kinds of oxygen atoms could be present on the $\mathrm{TiO}_{2}(110)$ surface, namely: threefold nearsurface oxygen atoms, which are identical to bulk ones $(530.4 \mathrm{eV})$; twofold ones, referred to as "bridging" oxygen atoms $(531.6 \mathrm{eV})[196,197]$ and single-fold ones, denoted "top" oxygen $(532.6 \mathrm{eV})$ [198-200]. The fourth peak at $533.4 \mathrm{eV}$ detected at $293 \mathrm{~K}$ can be attributed to adsorbed water [198]. The intensity of these XPS peaks (except that of bulk $\mathrm{O}$ ) decreased above $573 \mathrm{~K}$, due to the minor modification of the structure. "OH" like photoemission could emerge in the signal at $532.6-532.8 \mathrm{eV}$. This peak disappeared at $573 \mathrm{~K}$ in nanowires while in nanotubes it diminished only above $673 \mathrm{~K}$. The MS intensity of the peak for adsorbed water decreased significantly between 350 and $650 \mathrm{~K}$ (Fig. 10). Interestingly, around $700 \mathrm{~K}$ the photoemission at $533.5 \mathrm{eV}$ reappeared on nanotubes. This could be the result of $\mathrm{H}_{(\mathrm{a})}$ and $\mathrm{OH}_{(\mathrm{a})}$ recombination, but more probably we may attribute the photoemission at $533.3 \mathrm{eV}$ at $673 \mathrm{~K}$ to formyl- or formatelike species [201]. At this temperature a new carbon signal appeared around $291.0 \mathrm{eV}$ (not shown) that also supports the formation of formate-like species. Simultaneous DTG and MS measurements show minor amount of $\mathrm{CO}_{2}$ evolution above $650 \mathrm{~K}$ in the case of titanate nanotubes but not on nanowires. At $650-700 \mathrm{~K}$ the carbon signals (originated from carbon contamination) decreased significantly. All these experimental results indicate that there are significant differences in the structure and construction of the studied nanoobjects (e.g. nanotubes and nanowires).

\subsection{Diffuse reflectance infrared spectroscopy (DRIFTS)}

In Fig. 13 the temperature dependent FTIR spectra of titanate nanotubes and nanowires are displayed. On protonated nanowires and nanotubes the $\mathrm{OH}$ and $\mathrm{H}_{2} \mathrm{O}$ stretching vibrations [202,203] between 3000 and $3750 \mathrm{~cm}^{-1}$ can be detected up to $673 \mathrm{~K}$. The $\mathrm{OH}$ and $\mathrm{H}_{2} \mathrm{O}$ deformation signal [204] at $1618-1648 \mathrm{~cm}^{-1}$ is present up to $600-700 \mathrm{~K}$. The symmetric $\mathrm{H}_{2} \mathrm{O}$ vibration $\left(3652 \mathrm{~cm}^{-1}\right)$ disappeared at $673 \mathrm{~K}$ on nanowires. Interestingly, a very weak asymmetric infrared signal [205] attributed to $\mathrm{H}_{2} \mathrm{O}$ around $3730 \mathrm{~cm}^{-1}$ can be detected even at $773 \mathrm{~K}$ on nanotubes. At 1540 and $1430 \mathrm{~cm}^{-1}$ traces of a double signal can be detected also up to $773 \mathrm{~K}$, which could be assigned to the symmetric and asymmetric $\mathrm{C}-\mathrm{O}$ stretching of some oxygenated hydrocarbons and carboxylate-like species as contaminants.

\subsection{Raman spectroscopy on H-form titanate nanotubes and nanowires}

Raman spectra of pristine and heat treated titanate nanotubes and nanowires are presented in Fig. 14(A) and (B) together with the spectrum of a reference anatase sample. The spectra of as-synthesized nanostructures match bulk trititanate results where peaks in the $400-1000 \mathrm{~cm}^{-1}$ region can be assigned to Ti-O-Ti stretching vibrations [206,207]. The thermal behavior of the samples basically confirms the independent infrared spectroscopic, thermogravimetric and XRD measurements as well as other early literature findings insofar [208] as the trititanate structure appears to be deconstructed at approx. $573 \mathrm{~K}$. Annealing at higher temperatures initiates the trititanate

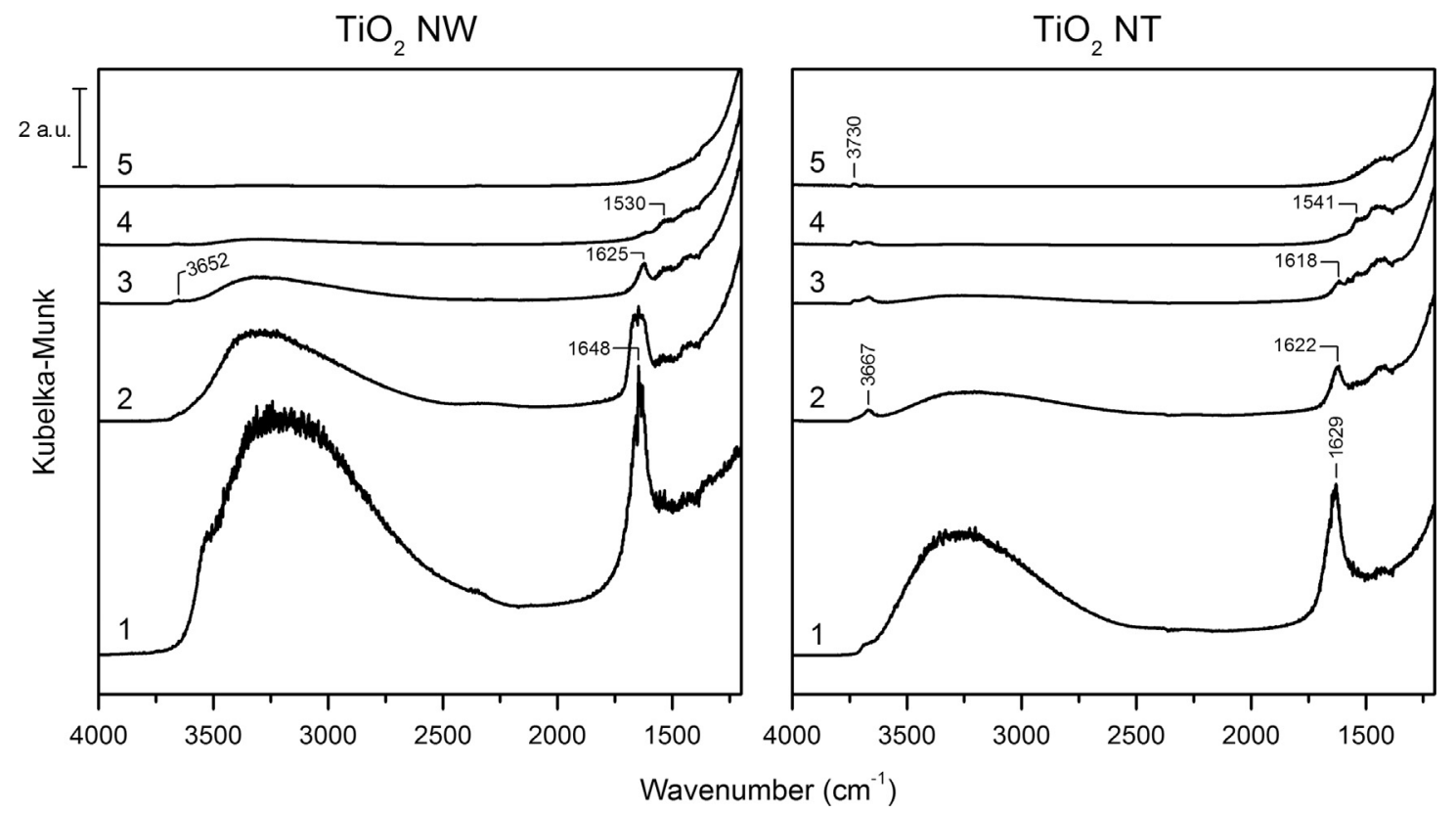

Fig. 13. FTIR spectra recorded after heat treatment for $60 \mathrm{~min}$ on nanowires (A) and nanotubes (B); $293 \mathrm{~K}$ (1), $473 \mathrm{~K}$ (2), $573 \mathrm{~K}$ (3), $673 \mathrm{~K}$ (4), and $773 \mathrm{~K}$ (5). All the spectra were measured at $293 \mathrm{~K}$ at a resolution of $2 \mathrm{~cm}^{-1}$, with 256 scan. Reproduced from Ref. [183]. 

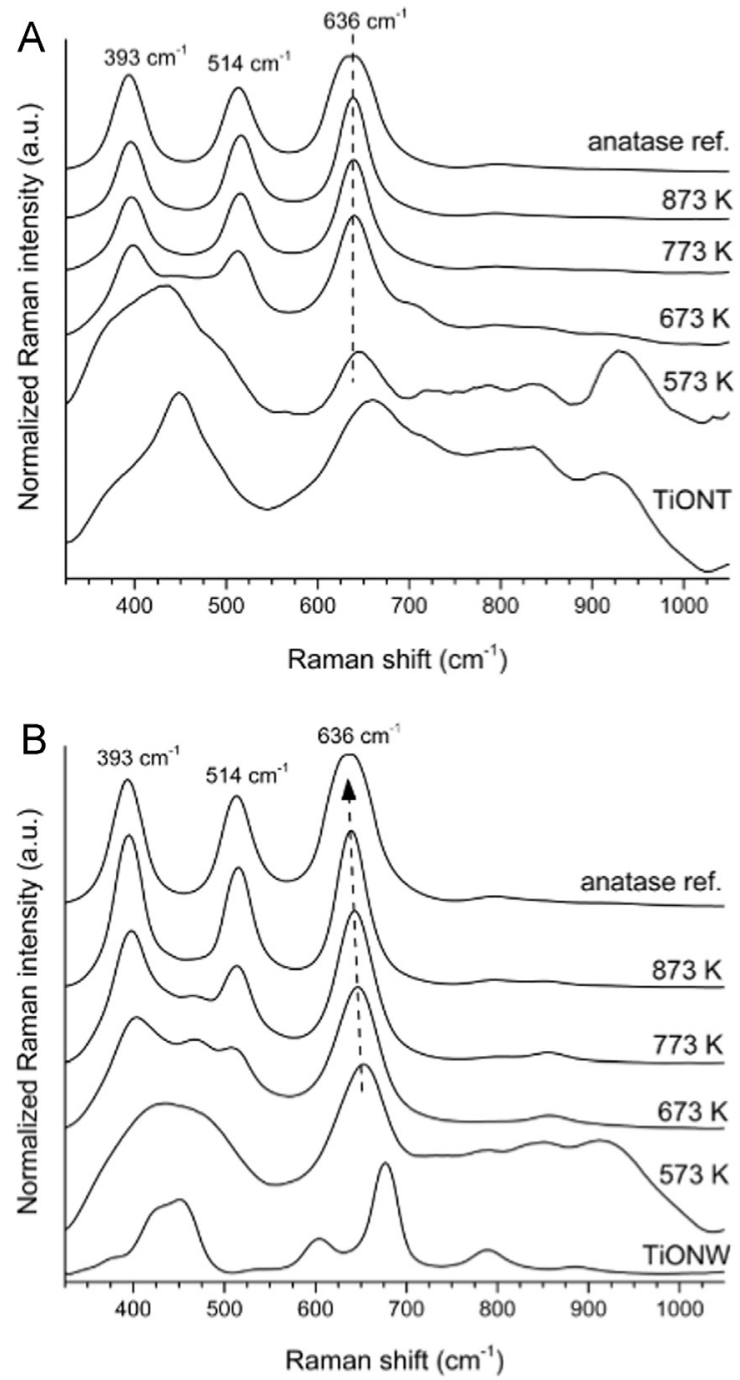

Fig. 14. Normalized Raman spectra of the thermal behavior of trititanate nanotubes (A) and nanowires (B). The spectra of as-synthesized samples are depicted at the bottom and the spectrum of a commercial anatase reference sample is shown at the top of each graph. Reproduced from Ref. [183].

to anatase conversion process which is concluded at $873 \mathrm{~K}$ as indicated by the good match between the $873 \mathrm{~K}$ spectrum and the reference anatase curve. Peaks at 393,514 and $636 \mathrm{~cm}^{-1}$ are assigned to the $\mathrm{B}_{1 \mathrm{~g}}, \mathrm{~A}_{1 \mathrm{~g}}$ and $\mathrm{E}_{2 \mathrm{~g}}$ modes of anatase, respectively [206].

A characteristic difference between the behavior of titanate nanotubes and nanowires is that in heat treated nanotubes the $E_{2 g}$ mode is found at exactly the anatase position $\left(636 \mathrm{~cm}^{-1}\right)$ from $573 \mathrm{~K}$ onwards, whereas in nanowires this mode experiences a gradual red shift from $648 \mathrm{~cm}^{-1}$ at $573 \mathrm{~K}$ to $636 \mathrm{~cm}^{-1}$ at $873 \mathrm{~K}$. A similar effect was observed by Du et al. [209] and Scepanovic et al. [210] in their in situ temperature-dependent Raman studies of nanocrystalline anatase. They argued that defects and nonstoichiometric composition could have a pronounced effect on the position of the soft $E_{g}$ modes. Adapting this argument to one-dimensional trititanates suggests that the thin and hollow structure of nanotubes is more easily converted to defect-free anatase than the bulky nanowires. This interpretation is further supported by XRD and ED measurements.

All these XPS, FTIR and Raman spectroscopy findings indicate that there are significant differences in the stability, surface structure and composition of the titanate nanowires and nanotubes. The surface structure is slightly affected by the preparation mode of the nanomaterials. These differences should be taken into account in the different application of these nanoobjects and in the explanations of the heterogeneous catalytic reaction mechanisms, where the acid or base active sites, defects concentration and surface area play important roles.

\subsection{Optical properties of $H$-form titanate nanowires and nanotubes; UV/vis spectrometry}

The optical properties of $\mathrm{TiO}_{2}$ anatase and rutil are welldocumented in the literature [3,211,212]. The knowledge of optical properties of $\mathrm{TiO}_{2}$-related materials (titania and titanates) is very important in photo-induced catalysis, photovoltaics, sensors and electronic devices and in many other applications. Generally, $\mathrm{TiO}_{2}$ is a wide-band gap semiconductor $\left(E_{g}=3.2 \mathrm{eV}\right)$ with indirect interband electron transition [213]. The photon absorption step in a photocatalytic reaction typically is viewed as a bulk (i.e., subsurface) process. However, two issues make this topic relevant for surfaces [3]. First, as a consequence of lattice truncation and formation of surface 'dangling' bond states, the electronic structures of surfaces are distinctly different than those of the bulk. Unique excitation events can arise from surface states or surface charge transfer complexes. Second, surface photon absortion processes can provide significant contribution to the overall photon absortion capacity of $\mathrm{TiO}_{2}$ nanoparticles.

It has been observed or proposed that quantum confinement in $\mathrm{TiO}_{2}$ nanoparticles in the $<10 \mathrm{~nm}$ size range results in a blue shift of the absortion threshold as the particles become more 'molecular' in character [214]. Titanium dioxide spheroidal nanoparticles show a relatively small apparent band gap blue shift $(<0.1-0.2 \mathrm{eV})$, caused by quantum size effects for particle size down to $2 \mathrm{~nm}[215,216]$. Such small effects are mainly due to the relatively high effective mass of carriers in $\mathrm{TiO}_{2}$ and the lowering of the exciton radius to the approximate range of $0.75-1.90 \mathrm{~nm}$. This implies that only very small particles could feature an increased band gap. It has also been suggested that such a small blue shift of band gap is due to the appearance of direct electronic transition of small particles, rather than the quantum confinement effect [217]. In the present state of the experimental data we may suppose that quantum confinement in $\mathrm{TiO}_{2}$ nanoparticles is more pronounced than direct electronic transition of small particles. The quantum confinement results in a significant blueshift mainly in very small clusters $(<2 \mathrm{~nm})$ [211].

The optical absortion spectra (UV/vis) of titanate nanowires and nanotubes have not been studied widely yet. The band gap energy $\left(E_{g}\right)$ was calculated according to Beranek and Kisch [218] who used the equation $\alpha=A\left(h \nu-E_{g}\right)^{n} / h \nu$, where $\alpha$ is the 
absorption coefficient, $A$ is a constant, $h \nu$ is the energy of light and $n$ is a constant depending on the nature of the electron transition [219]. Assuming an indirect band gap $(n=2)$ for $\mathrm{TiO}_{2}$ [220] with $\alpha$ proportional to $F\left(R_{\infty}\right)$, the band gap energy can be obtained from Kubelka-Munk $(\mathrm{K}-\mathrm{M})$ plots of $\left[F\left(R_{\infty}\right) /\right.$ $h \nu]^{1 / 2}$ vs. $h \nu$ as the interception at $\left[F\left(R_{\infty}\right) / h \nu\right]^{1 / 2}=0$ of the extrapolated linear part of the plot. The band gap for pure titanate nanowires and nanotubes was 3.10 and $3.07 \mathrm{eV}$, respectively [52]. A band gap of $3.06 \pm 0.03 \mathrm{eV}$ was measured for nanotubes when $\mathrm{TiCl}_{3}$ was used in preparation as a precursor [184]. In Fig. 15 some K-M function vs. $h \nu$ are displayed. For comparison the band gap determination in same circumstances and method for anatase (Hombicat) is also displayed; $3.17 \mathrm{eV}$ was obtained [221]. The band gap of nanosize anatase $\mathrm{TiO}_{2}$ is $3.20 \mathrm{eV}[3,222,223]$. The somewhat smaller band gap obtained for nanotubes and nanowires may reflect to the slight difference in the physico-chemical properties between nanostructered titanates and titania. It is plausible that more defects are created during the formation of titanates from titania by hydrothermal process.

Recently [224] a new form of $\mathrm{TiO}_{2}$ exhibiting a twodimensional structures with lepidocrocite $\mathrm{TiO}_{2}$ flat nanosheets was produced by exfoliation of layered protonic titanates [225]. The nanosheets of quasi- $\mathrm{TiO}_{2}$ offer several attractive features; anisotropic morphology with subnanometer scale thickness and well-defined composition and structure, because they are derived from a well-crystallized precursor synthesized at high temperatures. The absorption band for the colloidal nanosheets was significantly blue shifted $(<1.4 \mathrm{eV})$ relative to the absorption edge for the parent titanate and bulk $\mathrm{TiO}_{2}$ crystals, which may be attributed to size-quantization effect. The room temperature optical absorption and photoluminescence spectra for colloidal suspension are shown in Fig. 16. The presented data show that quantum confinement results in a significant blueshift also in titanates similar to nanosized $\mathrm{TiO}_{2}$.

The electronic band structure of these nanosheets that was revealed was compared with the calculated one [226]. It was shown that the band gap of nanosheets is strongly blue shifted $\left(E_{g}=3.84 \mathrm{eV}\right)$ relatively to the band gap of bulk $\mathrm{TiO}_{2}$, due to lower dimensionality, i.e., a 3D to 2D [227].

The highly ordered, vertically oriented $\mathrm{TiO}_{2}$ nanotube arrays are very good candidates for solar energy applications [228]. Uniform titania nanotube arrays of various pore size (22$110 \mathrm{~nm}$ ), length (200-6000 nm) and wall thickness (7-34 nm) are easily grown by tailoring the electrochemical synthesis conditions $[229,230]$. The physico-chemical properties of $\mathrm{TiO}_{2}$ arrays are very similar to the properties of $\mathrm{TiO}_{2}$ nanoparticles, due to the chemical and structural similarities. However, some unusual behavior of the nanotube array - resulting from the specific nanotubular morphology - is observed will be and considered now.

The band gap was determined from the Tauc plot which is presented in Fig. 17. The measured band gap is $3.34 \mathrm{eV}$. It may be noted that XRD results showed only anatase phase in the transparent titania nanotube array film [228]. The reported band gap value of anatase phase in bulk is $3.2 \mathrm{eV}[3,231]$. The slight blue shift in the value might be due to a quantization effect in the nanotubular film where the wall thickness is about $12 \mathrm{~nm}$. A band tail to $2.4 \mathrm{eV}$ is observed. The degree of lattice distortion is likely to be relatively higher for nanotube array films, thus causing aggregation of vacancies acting as trap states along the seams of nanotube walls leading to a lower band-to-band transition energy. Bandgap values of titanates prepared by different ways and doped with metals are collected in Table 3.

\section{Surface chemistry of titanate nanowires and nanotubes}

\subsection{Covalent functionalization of titanate nanostructures}

Wang et al. functionalized titanate nanotubes with straight aliphatic carboxylic acids of varying length and studied the interaction between the molecules and the nanotube by ARXPS [236]. They found that although nanotubes treated with stearic acid have lost some of their length, they conserved their original structure and morphology to a large extent. On the other hand, nanotubes treated with propionic acid have fragmented into multiple nanoparticles. X-ray diffraction studies indicated that while sampes treated with hexane, decane, tetradecane and hexadecane maintained their original crystal structure, the one subjected to propionic acid exhibited features characteristic of anatase.

A possible reason behind the shortening of stearic acid functionalized nanotubes is that the nanotube is partially dehydrated because of the bond formation between the carboxyl groups and the surface hydroxyl groups. Although this weakens the tube structure and increases the probability of fracture, the phenomenon can only take place on the tube surface because of the size of the stearic acid chain. Therefore, the tubular morphology is maintained. On the other hand, the size of a propionic acid molecule is less than $0.5 \mathrm{~nm}$. Comparing this value with the titanate interlayer distance of $0.7-0.9 \mathrm{~nm}$ and the nanotube inner diameter of $3-5 \mathrm{~nm}$ reveals that propionic acid can penetrate the whole tube structure and bond to any $\mathrm{OH}$ groups, thus cutting the nanotube into small units that eventually recrystallize as anatase nanoparticles.

The adsorption of organic molecules on metal oxide surfaces increases the intenstity of carbon XPS peaks and reduces the intensity of metal and oxygen related peaks. However, in titanate nanotubes the adsorption of aliphatic carboxylic acids increased the intensity of all relevant peaks (C 1s, Ti $2 p$ and O 1s) (Fig. 18). The largest intensities occured in the sample subjected to propionic acid initiated recrystallization. This could be explained by the increasing total density of the sample. The C 1s XPS peak could be decomposed into 4 components if the carboxylic acid chain length was 3 or $6(284.8 \mathrm{eV}$ for $\mathrm{C}-\mathrm{C}$, $286.2 \mathrm{eV}$ for $\mathrm{C}-\mathrm{O}, 288.3 \mathrm{eV}$ for $\mathrm{O}-\mathrm{C}-\mathrm{O}$ or $\mathrm{C}=\mathrm{O}$ and $288.9 \mathrm{eV}$ for the $\mathrm{O}=\mathrm{C}-\mathrm{O}$ bonded carbon), whereas in the case of longer aliphatic chains the $\mathrm{C}_{3}$ peak was not detectable [237].

Nanotubes functionalized with caprylic acid or even longer chain length carboxylic acids were also subjected to ARXPS analysis done in $30^{\circ}$ and $60^{\circ}$ photoemission angle. The C3 peak became visible at $30^{\circ}$ in caprylic acid and tetradecanoic acid functionalized titanate nanotubes. The authors concluded 
(A)

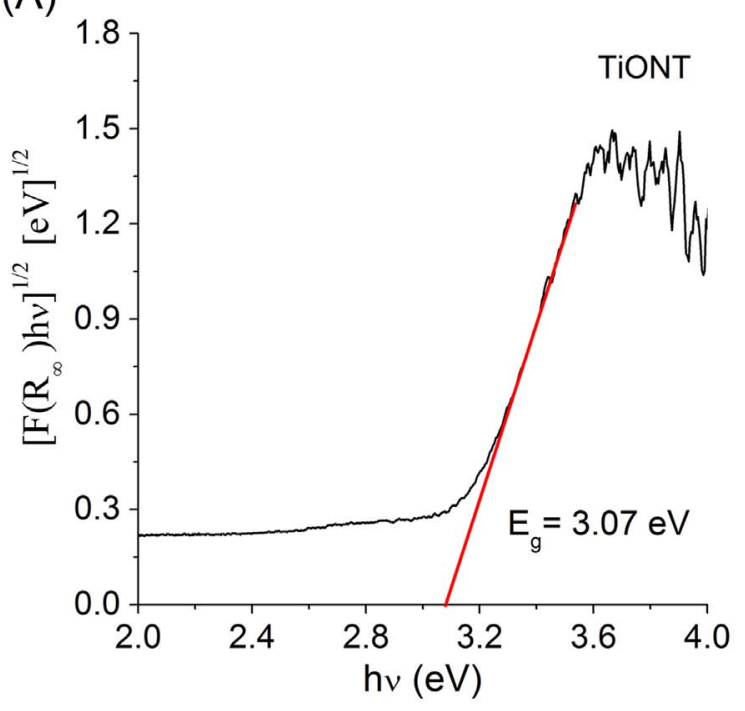

(B)

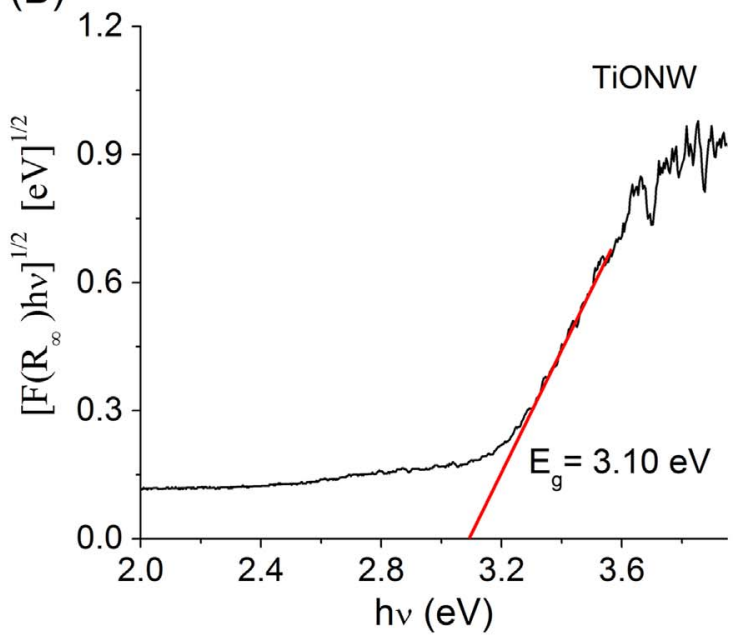

(C)

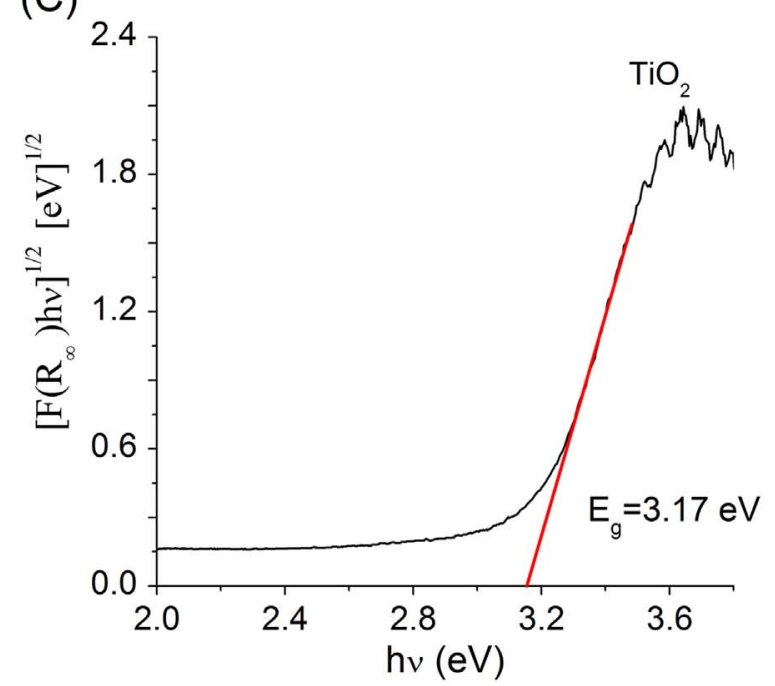

Fig. 15. Kubelka-Munk function vs. wavelength of $\mathrm{H}$-form titanate nanotubes (TiONT) (A), nanowires (TiONW) (B) and high surface area of anatase (Hombicat; $300 \mathrm{~m}^{2} / \mathrm{g}$ ) (C). that a chain length of 14 or less results in either mono- or bidentate carboxylic acid binding to the titanate surface, whereas chains longer than 16 carbon atoms always bind as monodentate ligands [236].

Bond formation between the titanate nanotube and carboxylic acids was also suggested on the basis of thermogravimetric measurements. H-form titanate nanotube lose water in two steps: physisorbed water is released below $100{ }^{\circ} \mathrm{C}$ and interlayer water is released above this temperature. However, a third step appears in the TG curve in the $500-650{ }^{\circ} \mathrm{C}$ range for carboxylic acid functionalized titanate nanotubes, and this temperature is characteristic of the corresponding carboxylates [236].

Wang and co-workers synthesized titanate nanotubes modified with benzoic acid by mixing $\mathrm{H}$-form titanate nanotubes (HTNT) with benzoic acid (BA) in different ratios [238]. Their objective was to create a monomolecular BA layer on the titanate surface. No benzoic acid reflections were observable in the XRD patterns (Fig. 19) up to $0.350 \mathrm{~g} \mathrm{BA} / \mathrm{g}$ HTNT. Peaks assignable to crystalline $\mathrm{BA}$ indicated benzoic acid excess above this ratio. Monomolecular coverage BA/HTNT ratio was calculated from the area ratio of the BA (200) and HTNT (110) reflections. The intensity ratio (Fig. 19, right panel) indicates the relative amount of benzoic acid excess. Extrapolating a linear fit of the nonzero points to the abscissa yielded $0.305 \mathrm{~g} \mathrm{BA} / \mathrm{g}$ HTNT as the BA loading necessary for monomolecular coverage.

Three possible binding modes were suggested to occur between BA and the HTNT surface: monodentate, bridged bidentate and chelate bidentate (Fig. 20, left).

XPS measurements confirmed the formation of carboxylate ions in the reaction between the carboxylic acid and the surface $\mathrm{OH}$ groups of HTNT. The amount of benzoic acid necessary for monomolecular coverage was calculated by plotting the intensity of the $\mathrm{C}_{4}$ component of the $\mathrm{C} 1 \mathrm{~s}$ XPS peak as a function of BA/HTNT ratio (Fig. 20, right). Data points fell onto two lines that intersected at $0.274 \mathrm{~g} \mathrm{BA} / \mathrm{g}$ HTNT ratio, which is in good agreement with the monomolecular coverage calculated from XRD.

Huskic and co-workers functionalized titanate nanotubes by silanes using 3-(aminopropyl)-triethoxysilane (APTES), triethoxyoctylsilane, 3-glycidyloxypropyl trimethoxysilane and 3-aminopropylmethyl diethoxysilane [239]. Their objective was to improve the load transfer in titanate nanotube reinforced epoxy resin nanocomposites. A detailed account of the FT-IR spectroscopy of the APTES-based materials was published. A band at around $900 \mathrm{~cm}^{-1}$ indicated organicinorganic bond formation that could be either due to $\mathrm{Ti}-\mathrm{O}-\mathrm{Si}$ bonds or alternatively, to $\mathrm{Si}-\mathrm{O}$ or $\mathrm{Si}-\mathrm{OH}$ groups $[240,241]$. The functionalized samples were heat treated at 500 and $800{ }^{\circ} \mathrm{C}$. Infrared spectra recorded after the high temperature annealing still featured the characteristic organic-inorganic peaks at 900 and $940 \mathrm{~cm}^{-1}$, thus the presence of Si-O-Si and Ti-O-Si bonds was confirmed. 


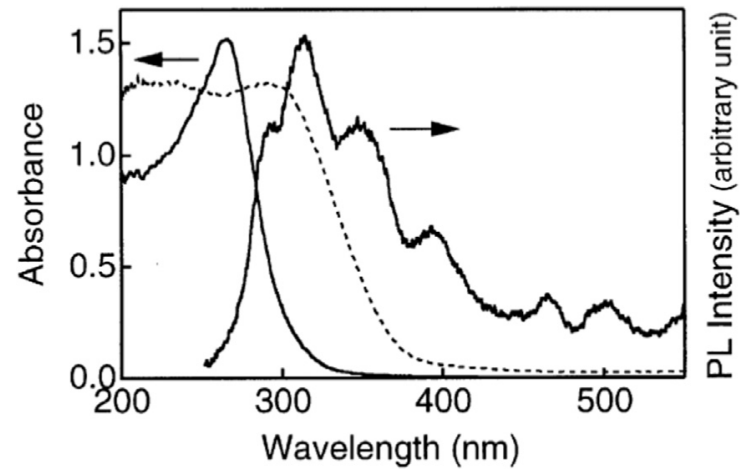

Fig. 16. Room-temperature optical absorption and photoluminescence spectra from the colloidal suspension. Reproduced from Ref. [189].

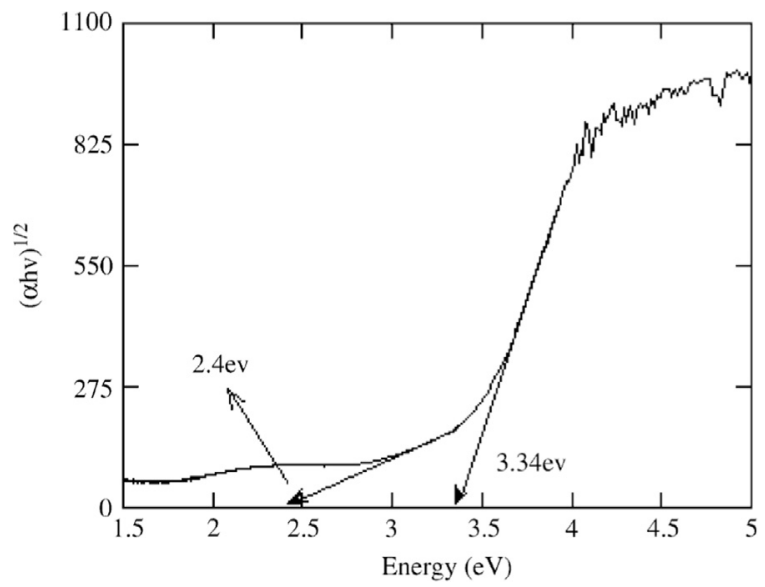

Fig. 17. Tauc plot of $450{ }^{\circ} \mathrm{C}$ annealed nanotubes array film. An indirect band gap and band tailing up to $2.4 \mathrm{eV}$ are observed in the film. Reproduced from Ref. [228].

Table 3

Bandgap of titanates prepared by different ways and doped with metals ${ }^{\mathrm{a}}$.

\begin{tabular}{llll}
\hline Different forms of titanates & \multicolumn{2}{l}{ Bandgap $(\mathbf{e V})$} & References \\
\cline { 2 - 3 } & Nanotubes & Nanowires & \\
\hline H-form $^{\mathrm{b}}$ & 3.10 & $3.07(3.14)$ & {$[52,280]$} \\
& 3.06 & - & {$[184]$} \\
& 3.20 & - & {$[232]$} \\
& 3.40 & - & {$[266]$} \\
& 3.30 & - & {$[233]$} \\
Na-form & $2.94-3.31$ & - & {$[363]$} \\
Co-doped & $3.23-3.51$ & - & {$[363]$} \\
& - & 2.41 & {$[280]$} \\
Fe-doped & $2.36-2.37$ & - & {$[234]$} \\
Ag-doped & 3.05 & - & {$[266]$} \\
Au-doped & 3.2 & - & {$[233]$} \\
TiO & array \\
$2 \mathrm{D}$ titanate nanosheets & 2.67 & 2.84 & {$[52]$} \\
& 3.34 & - & {$[228,235]$} \\
\hline
\end{tabular}

${ }^{\mathrm{a}}$ For comparison the same corresponding literature should be taken into account.

${ }^{\mathrm{b}}$ Prepared by hydrothermal conversion.

${ }^{\mathrm{c}}$ Prepared by electrochemical method.

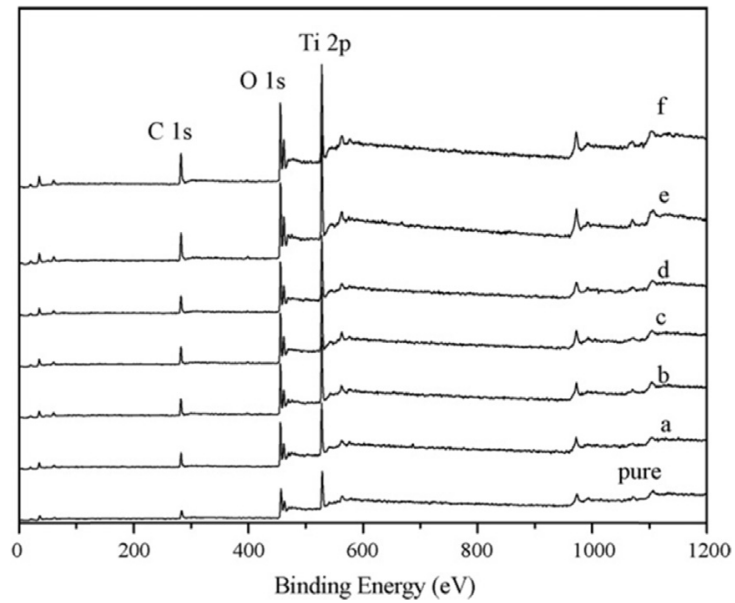

Fig. 18. XP spectra of $\mathrm{H}$-form titanate nanotubes (pure) and nanotubes surface functionalized by metacetonic acid (a), caproic acid (b), capric acid (c), tetradecanoic acid (d), necadecanoic acid (e) and stearic acid (f). Reproduced from Ref. [236].

Wang et al. used the aminosilane reagent ([1-(2-amino-ethyl)3-aminopropyl]trimethoxysilane (AAPTS)) to functionalize titanate nanotubes in order to improve their $\mathrm{Cr}(\mathrm{VI})$ adsorption properties [242]. The ion adsorption capacity of the resulting $\mathrm{NH}_{2}$-TNT samples went up to $153.85 \mathrm{mg} / \mathrm{g}$ from the starting value of $26.60 \mathrm{mg} / \mathrm{g}$ characteristic of the unfunctionalized nanotubes. The characteristic (200) titanate peak located at around $10^{\circ} 2 \Theta$ suffered only a minimal shift during the functionalization. This indicates that the AAPTS did not penetrate the interlayer space and the crystalline structure of the titanate walls remained intact. An FT-IR study on the bonding between the AAPTS and the HTNT revealed that both $\mathrm{Si}-\mathrm{O}-\mathrm{Ti}$ (shoulder at $945 \mathrm{~cm}^{-1}$ ) and $\mathrm{Si}-\mathrm{O}-\mathrm{Si}$ (peak at $1039 \mathrm{~cm}^{-1}$ ) bonds were formed during the reaction (Fig. 21). Therefore, the authors suggested that the polymerized network of aminosilane molecules is covalently bound to the titanate nanotube surface. The hydrolysis of the methoxy groups of the AAPTS is thought to initiate the reaction, which then proceeds by the condensation reaction between silanol $-\mathrm{OH}$ groups and surface Ti-OH groups [243].

Liu et al. synthesized polyethylene-imine (PEI) functionalized titanate nanotubes by reacting the amino groups with the strongly Brönsted acidic HTNT surface [244]. They investigated the surface acidity of HTNTs by the $\mathrm{NH}_{3}$ adsorption in situ DRIFT technique. Absorption peaks at 1188, 1228 and $1594 \mathrm{~cm}^{-1}$ were assigned to ammonia coordinated to Lewis acid sites, whereas the intense peaks at 1460 and $1417 \mathrm{~cm}^{-1}$ and the broad feature at $1814 \mathrm{~cm}^{-1}$ indicated the presence of $\mathrm{NH}_{4}^{+}$ions, i.e. Brönsted acidic centers. Only Lewis acid sites were found in the spectra of the reference P25 and MCM-41 materials. The acidity of PEI functionalized TNT was investigated as well. The intensity of peaks corresponding to both Lewis and Brönsted acid sites decreased with increasing PEI content, which is most likely caused by the bond formation between the acid sites and the amine groups. It was also possible to identify the characteristic peak of the $\mathrm{RNH}_{3}^{+}$group 
that is formed by the reaction between surface - $\mathrm{OH}$ groups and the $\mathrm{RNH}_{2}$ amine. The intensity of this peak increased with the amount of PEI added to the system (Fig. 22).
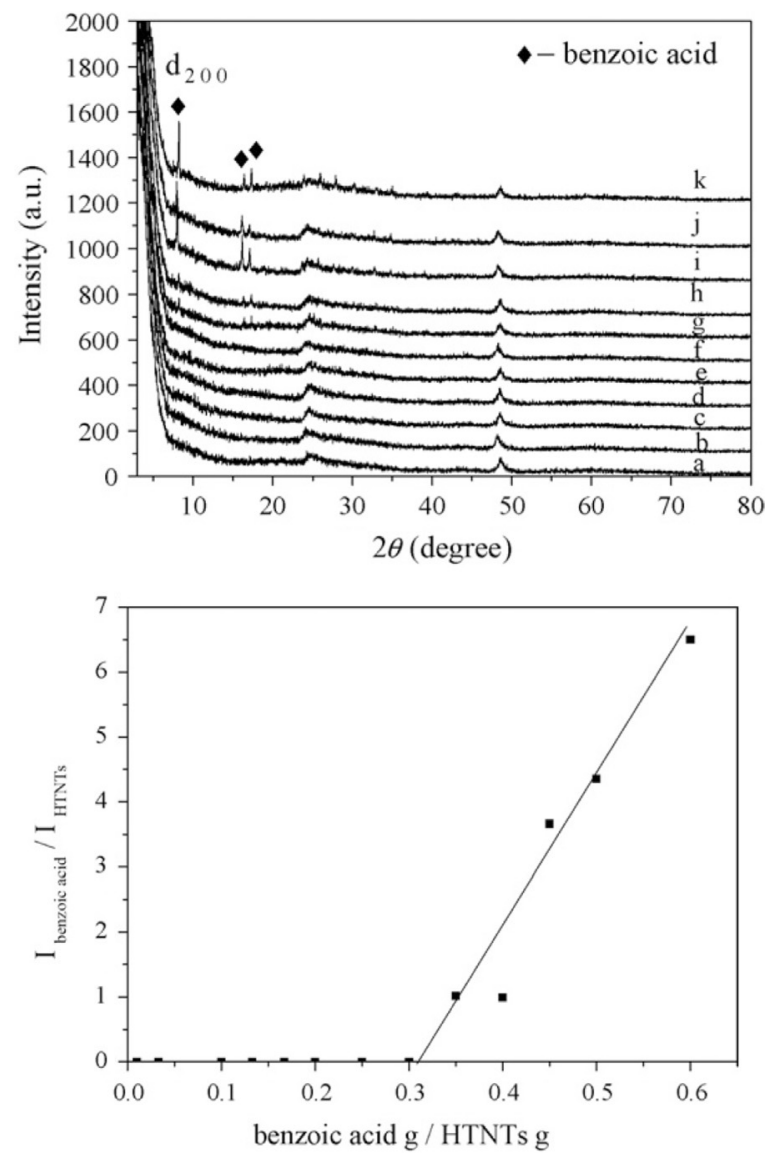

Fig. 19. Left: XRD patterns of BA/HTNT samples with different BA loadings (g/g): 0.033 (a), 0.100 (b), 0.133 (c), 0.200 (d), 0.250 (e), 0.300 (f), 0.350 (g), $0.400(\mathrm{~h}), 0.450(\mathrm{i}), 0.500(\mathrm{j})$, and $0.600(\mathrm{k})$. Right: linear extrapolation of the BA excess yields the BA/TNT ratio $(\mathrm{g} / \mathrm{g})$ corresponding to monomolecular coverage. Reproduced from Ref. [238].

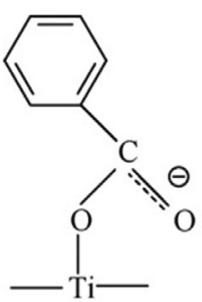

(a)

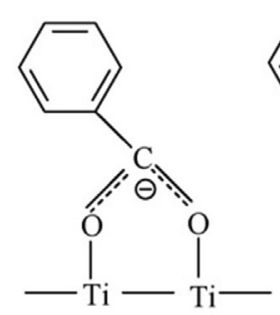

(b)

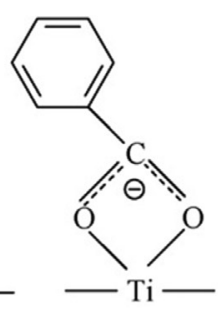

(c)

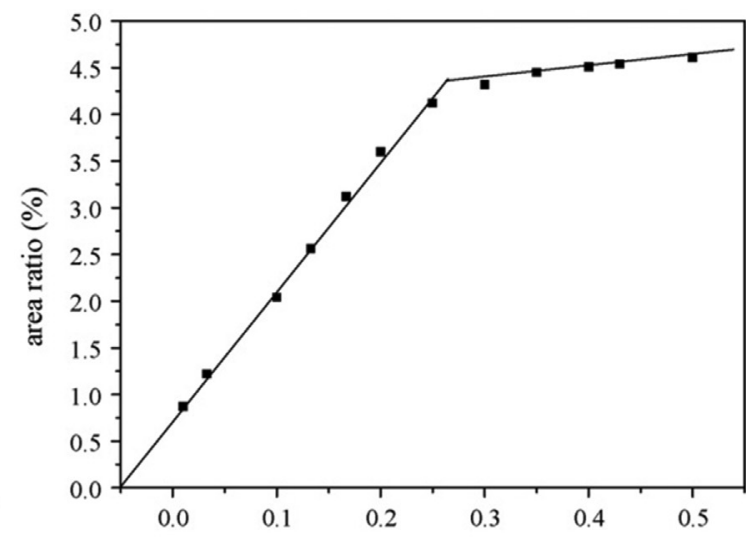

BA g / HTNTs g

\subsection{Metal loading to titanate nanotubes (TiONT)}

Titanate nanostructures are of great interest for catalytic applications because their high surface area and cation exchange capacity provides the possibility of achieving a high metal dispersion.

Ion exchange allows titanate nanostructures to incorporate metal adatoms in their framework, which may create another type of active center besides metal clusters. Since titanate nanotubes are characterized by good ion-exchange properties $[245,246]$, one method of catalyst deposition involves preliminary ion-exchange of the catalyst precursor in its cationic form with protons within nanotubular titanates. This allows an atomic-scale distribution of metal cations in the titanate lattice, thus achieving a higher metal loading compared to the simple adsorption of the precursor on the surface. Studies in this direction are still scarce in the literature because of the genuine novelty of the titanate nanomaterial based photocatalysis and low-temperature thermal heterogeneous catalysis field.

\subsubsection{Effect of ion exchange on the surface properties of titanate nanostructures}

The negative charge of the titanic acid framework makes titanate nanostructures rather capable cation exchange materials. The theoretical cation exchange capacity (CEC) of sodium trititanate is $5.8 \mathrm{mmol} / \mathrm{g}(5.8 \mathrm{meq} / \mathrm{g})$ for monovalent cations and $2.9 \mathrm{mmol} / \mathrm{g}$ for divalent cations (calculated with $10 \mathrm{wt} \%$ structural water content). This compares favorably with the $5.48 \mathrm{meq} / \mathrm{g}$ CEC value of the extensively used LTA zeolite [247]. Table 4 presents representative CEC values determined experimentally for various titanates. Since the structure collapses upon ion exchange with most bivalent and trivalent cations (e.g. $\mathrm{Hg}^{2+}, \mathrm{Pb}^{2+}, \mathrm{Cr}^{3+}$ ), the ion exchange process is often irreversible. This is the likely reason why literature data on transition metal ion exchange is surprisingly scarce for titanate nanostructures. We shall now discuss the effect of ion exchange on titanate surface chemistry in detail.

Wada et al. studied the effect of synthesis parameters on the structure and surface properties of titanate nanotubes [65].

Fig. 20. Left: possible binding modes of benzoic acid to the H-form titanate nanotube surface. Right: determination of the BA/HTNT ratio corresponding to monomolecular coverage from XPS data. Reproduced from Ref. [238]. 


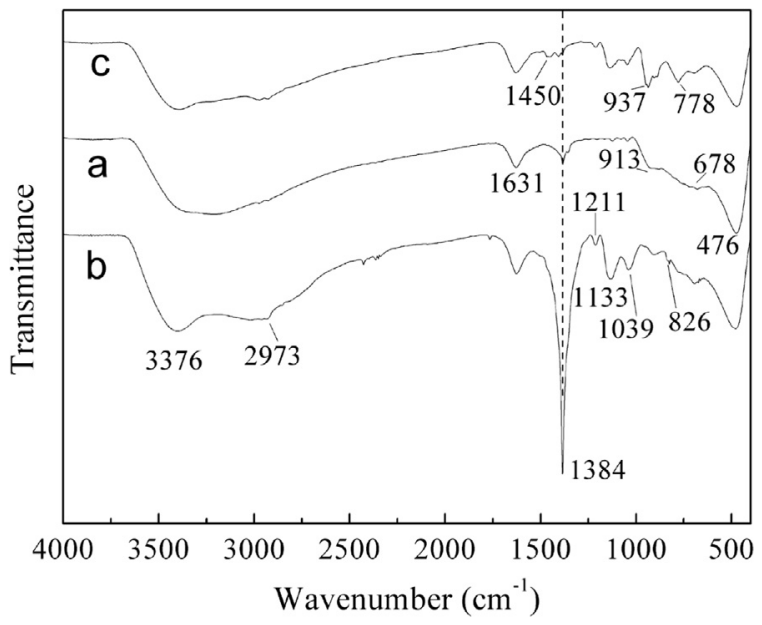

Fig. 21. FT-IR spectra of (a) HTNT, (b) $\mathrm{NH}_{2}-\mathrm{THT}$ and (c) $\mathrm{Cr}$ (IV)-laden $\mathrm{NH}_{2}$-TNT. Reproduced from Ref. [242].

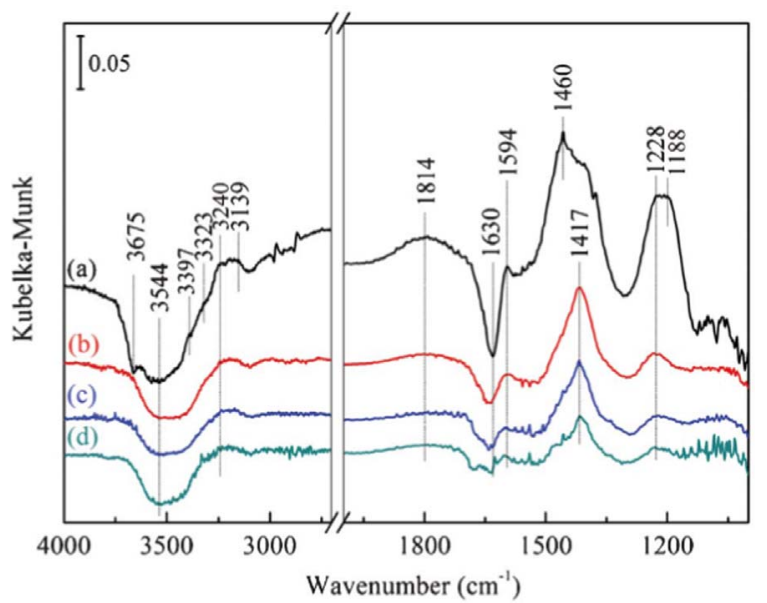

Fig. 22. DRIFT spectra of $\mathrm{NH}_{3}$ adsorption saturation on (a) titanate nanotubes and PEI-functionalized titanate nanotubes with different amine loading, (b) PEI-TNT-20, (c) PEI-TNT-30, (d) PEI-TNT-50 at $100{ }^{\circ} \mathrm{C}$. Reproduced from Ref. [244].

They mixed $5 \mathrm{~g} \mathrm{TiO}_{2}$ into $70 \mathrm{ml} 10 \mathrm{M} \mathrm{NaOH}$ solution and run the hydrothermal synthesis for $20 \mathrm{~h}$ at 50,150 or $200{ }^{\circ} \mathrm{C}$, then protonated the product by washing it with $0.1 \mathrm{M} \mathrm{HNO}_{3}$ solution. Their XRD studies indicated that $50{ }^{\circ} \mathrm{C}$ was not enough to convert the anatase particles into nanotubes, but the edges of the 20-30 nm diameter $\mathrm{TiO}_{2}$ particles have shown the signs of partial dissolution already. Reactions at $150{ }^{\circ} \mathrm{C}$ and $200{ }^{\circ} \mathrm{C}$ yielded titanate nanotubes and nanowires, respectively.

Surface acidity was studied by pyridine adsorption monitored by FT-IR spectroscopy. The spectra of the reference $\mathrm{TiO}_{2}$ as well as those of the hydrothermal products feature a peak at around $1450 \mathrm{~cm}^{-1}$ when subjected to pyridine vapor. This indicates the presence of pyridine coordinated to Lewis acid sites, in this particular case most likely to coordinatively unsaturated $\mathrm{Ti}^{4+}$ centers $[255,256]$. Interestingly, the $1540 \mathrm{~cm}^{-1}$ peak characteristic of pyridine adsorbed on Brönsted acid sites appeared only in the case of the titanate nanotubes synthesized at $150{ }^{\circ} \mathrm{C}$ (Fig. 23).
Table 4

Representative cation exchange capacities (CEC) of titanate nanostructures for different cations.

\begin{tabular}{|c|c|c|c|}
\hline Titanate structure & CEC (meq/g) & Cation & Reference \\
\hline $\mathrm{Na}_{2} \mathrm{Ti}_{3} \mathrm{O}_{7}$ & \multicolumn{3}{|c|}{5.8 (theoretical limit) } \\
\hline $\mathrm{Na}_{2} \mathrm{Ti}_{3} \mathrm{O}_{7}$ & 0.2 & $\mathrm{Na}^{+}$ & [64] \\
\hline \multirow{2}{*}{$\mathrm{Na}_{2} \mathrm{Ti}_{3} \mathrm{O}_{7}$} & 5.44 & $\mathrm{Sr}^{2+}$ & \multirow[t]{2}{*}{ [248] } \\
\hline & 0.96 & $\mathrm{Cs}^{+}$ & \\
\hline $\mathrm{Na}_{2} \mathrm{Ti}_{3} \mathrm{O}_{7}$ & 1.26 & $\mathrm{Sr}^{2+}$ & [249] \\
\hline \multirow{3}{*}{$\mathrm{H}_{2} \mathrm{Ti}_{3} \mathrm{O}_{7}$} & $0.03(1.3)$ & $\mathrm{NaCl}(\mathrm{NaOH})$ & \multirow{3}{*}[65]{} \\
\hline & $0.21(2.9)$ & $\mathrm{NaCl}(\mathrm{NaOH})$ & \\
\hline & $0(4.4)$ & $\mathrm{NaCl}(\mathrm{NaOH})$ & \\
\hline \multirow[t]{7}{*}{$\mathrm{H}_{2} \mathrm{Ti}_{3} \mathrm{O}_{7}$} & 2.625 & $\mathrm{Na}^{+}$ & \multirow[t]{7}{*}{ [250] } \\
\hline & 2.409 & $\mathrm{~K}^{+}$ & \\
\hline & 2.021 & $\mathrm{Cs}^{+}$ & \\
\hline & 1.637 & $\mathrm{NH}_{4}^{+}$ & \\
\hline & 1.732 & $\mathrm{Mg}^{2+}$ & \\
\hline & 1.735 & $\mathrm{Ca}^{2+}$ & \\
\hline & 1.965 & $\mathrm{Ba}^{2+}$ & \\
\hline $\mathrm{Na}_{2} \mathrm{Ti}_{2} \mathrm{O}_{4}(\mathrm{OH})_{2}$ & 3.20 & $\mathrm{UO}_{2}^{2+}$ & {$[251]$} \\
\hline $\mathrm{Na}_{4} \mathrm{Ti}_{9} \mathrm{O}_{20}$ & 5.30 & $\mathrm{Sr}^{2+}$ & {$[252]$} \\
\hline $\mathrm{Na}_{1.5} \mathrm{H}_{0.5} \mathrm{Ti}_{3} \mathrm{O}_{7}$ & 1.14 & $\mathrm{Ba}^{2+}$ & \\
\hline $\mathrm{Na}_{x} \mathrm{Ti}_{2-x / 4} \square_{x / 4} \mathrm{O}_{4}(x \approx 1)$ & 3.80 & $\mathrm{Sr}^{2+}$ & {$[253]$} \\
\hline \multirow[t]{5}{*}{$\mathrm{H}_{0.7} \mathrm{Ti}_{1.825} \square_{0.175} \mathrm{O}_{4} \cdot \mathrm{H}_{2} \mathrm{O}$} & 2.95 & $\mathrm{Li}+$ & \multirow[t]{5}{*}{ [254] } \\
\hline & 2.92 & $\mathrm{Na}+$ & \\
\hline & 2.45 & $\mathrm{~K}+$ & \\
\hline & 2.32 & $\mathrm{Rb}+$ & \\
\hline & 1.93 & $\mathrm{Cs}+$ & \\
\hline
\end{tabular}

Camposeco et al. modified trititanate nanotubes with $\mathrm{V}_{2} \mathrm{O}_{5}$ and $\mathrm{WO}_{3}$ and tested the resulting materials in the selective catalytic reduction of $\mathrm{NO}$ with $\mathrm{NH}_{3}$ (SCR-NH 3 process) [257]. Their catalysts were prepared by the wet impregnaton of protonated titanate nanotubes with aqueous $\mathrm{NH}_{4} \mathrm{VO}_{3}$ and $\mathrm{H}_{2} \mathrm{WO}_{4}$ solutions and labeled as V10/TAN (10 $\left.\mathrm{wt} \% \mathrm{~V}_{2} \mathrm{O}_{5}\right)$ and V5-W3/TAN (5 wt $\% \mathrm{~V}_{2} \mathrm{O}_{5}+3 \mathrm{wt} \% \mathrm{WO}_{3}$ ) (Table 5).

No V-containing phase could be identified in the V10/TAN catalyst by HR-TEM and HR-TEM-STEM, whereas in V5W3/TAN clusters measuring approx. $1 \mathrm{~nm}$ in diameter were clearly visible on the nanotube surfaces. The high dispersity of the material prevented the XRD identification of the V- or Wcontaining phases, but the oxidation state of the metals could be determined by XPS. High intensity peaks in V10/TAN spectra revealed that vanadium was present as $\mathrm{V}^{4+}\left(\mathrm{VO}_{2}\right)$ és $\mathrm{V}^{5+}\left(\mathrm{V}_{2} \mathrm{O}_{5}\right)$ on the surface. Vanadium was not visible in the spectra of the V5-W3/TAN sample and $\mathrm{W}$ was found to be in +6 oxidation state like in $\mathrm{WO}_{3}$. Ti $2 \mathrm{p}$ peaks shifted towards higher energies in the $\mathrm{V}$ - and $\mathrm{W}$-containing samples, which was interpreted as an indication of strong interaction between the $\mathrm{V}$ - and $\mathrm{W}$-based particles and the titanate nanotube support (Table 6).

Surface acidity was studied by in situ pyridine FT-IR. V and $\mathrm{W}$ modification affected the amount of available acid sites. The concentration of Lewis centers was increased compared to that measured on protonated titanate nanotubes. Brönsted centers, on the other hand, increased in numbers only in the $10 \mathrm{wt} \% \mathrm{~V}$ 


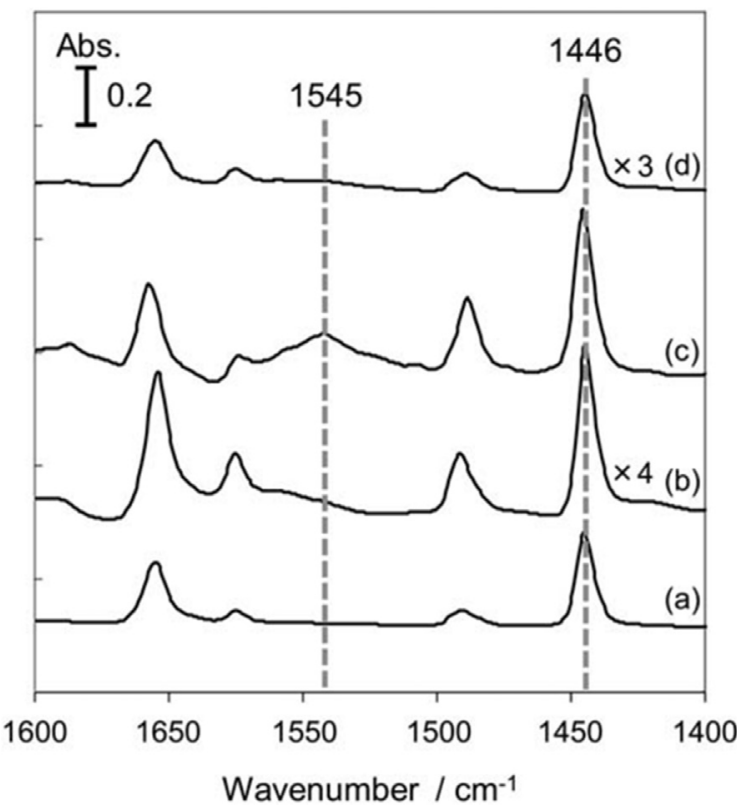

Fig. 23. Different FT-IR spectra for pyridine adsorbed on (a) $\mathrm{TiO}_{2}$, (b) HT-Ti-323, (c) HT-Ti-423, and (d) HT-Ti-473. Reproduced from Ref. [65].

containing samples. Their amount decreased upon adding $\mathrm{W}$ to the system, however, the strength of the Brönsted sites increased simultaneously so much that Brönsted-bound pyridine was identifiable in the IR spectrum even after heating to $400{ }^{\circ} \mathrm{C}$ after adsorption. NO conversion at $400{ }^{\circ} \mathrm{C}$ was $52 \%$ over unmodified nanotubes and $93 \%$ on the V10/TAN sample. The promotor effect of $\mathrm{WO}_{3}$ was not observable on V5-W3/ TAN: it offered NO conversion very similar to that of V10/ TAN.

Morgado studied iron-exchanged titanate nanotubes synthesized by mixing Na-form titanate nanotubes in aqeuous $\mathrm{Fe}$ $\left(\mathrm{NO}_{3}\right)_{3}$ solutions of different concentration, aiming at obtaining 2, 5, 10, 15 and $30 \mathrm{wt} \%$ iron loading [258]. TEM and XRD confirmed that the structure and morphology of the nanotubes remained largely intact. The characteristic titanate reflection at $10^{\circ} 2 \Theta$ shifted upwards irrespective of the iron loading, thus indicating a slight contraction of the titanate layers upon ion exchange. The optical absorption of the modified nanotubes shifted towards the visible spectral region according to diffuse reflectance UV-vis measurements. Spectra were analyzed in the Kubelka-Munk representation to determine the band gap, which was found to decrease with increasing iron content from $3.5 \mathrm{eV}$ to $2.4 \mathrm{eV}$.

The Ti $2 \mathrm{p}_{3 / 2}$ XPS peak appeared in the $458.5-458.9 \mathrm{eV}$ range indicating that $\mathrm{Ti}$ was four-coordinated in all samples. Two characteristic oxygen peaks corresponding to $\mathrm{Ti}^{4+}$ bound bridge $\mathrm{O}$ and surface terminal $\mathrm{OH}$ were also identified.

The ratio of $\mathrm{Fe}^{2+}(709.6 \mathrm{eV})$ and $\mathrm{Fe}^{3+}(711.2 \mathrm{eV})$ iron can be calculated by deconvoluting the $\mathrm{Fe} 2 \mathrm{p}_{3 / 2}$ and $\mathrm{Fe} 2 \mathrm{p}_{1 / 2}$ XPS peaks. The $\left[\mathrm{Fe}^{2+}\right] /\left[\mathrm{Fe}^{3+}\right]$ appears to decrease with increasing iron content in iron-exchanged titanate nanotubes. While at $2 \%$ $\mathrm{Fe}$ loading all iron is in $\mathrm{Fe}(\mathrm{II})$ state, increasing the iron contents to $15 \mathrm{wt} \%$ results in $67 \%$ of the iron being Fe(III).
Table 5

Properties and preparation method of the catalysts employed by the SCR-NH process. Reproduced from Ref. [257].

\begin{tabular}{|c|c|c|c|c|c|}
\hline Catalyst & $\begin{array}{l}\mathrm{V}_{2} \mathrm{O}_{5} \\
(\mathrm{wt} \%)\end{array}$ & $\begin{array}{l}\mathrm{WO}_{3} \\
(\mathrm{wt} \%)\end{array}$ & $\begin{array}{l}\text { Preparation } \\
\text { method }\end{array}$ & $\mathrm{NaOH}, N$ & $\begin{array}{l}\text { Annealed } \\
\text { temp. }\left({ }^{\circ} \mathrm{C}\right)\end{array}$ \\
\hline TAN & - & - & Hydrothermal & 10 & 450 \\
\hline V10/TAN & 10 & - & Hydrothermal & 10 & 450 \\
\hline $\begin{array}{l}\text { V5-W3/ } \\
\text { TAN }\end{array}$ & 5 & 3 & Hydrothermal & 10 & 450 \\
\hline $\begin{array}{l}\text { V5-W3/ } \\
\text { TAN- } \\
500{ }^{\circ} \mathrm{C}\end{array}$ & 5 & 3 & Hydrothermal & 10 & 500 \\
\hline $\begin{array}{l}\text { V5-W3/ } \\
\mathrm{TiO}_{2}\end{array}$ & 5 & 3 & $\begin{array}{l}\text { Wet } \\
\text { impregnation }\end{array}$ & - & 450 \\
\hline
\end{tabular}

\subsubsection{Metal nanoparticles on titanate nanostructures}

Nanoparticles of different metals ( $\mathrm{Pt}, \mathrm{Pd}, \mathrm{Au}, \mathrm{Ni})$ or a metal hydroxide $(\mathrm{Ru})$ were immobilized on the surface of mesoporous titanate nanotubes produced by the alkali hydrothermal treatment of $\mathrm{TiO}_{2}$, and have been characterized by HRTEM [32,259]. Two different approaches were utilized for the deposition of metal particles into the internal pores of titanate nanotubes: (i) deposition from solution confined inside the nanotubes and (ii) blocking the external surface of the nanotubes. A third method, ion-exchange of protons onto metal cations in titanate nanotubes followed by reduction or alkali treatment (in the case of Ru hydroxide), has been used for deposition of metal nano-particles on both the internal and external surfaces of the nanotubes. Metal or metal hydroxide nanoparticles deposited by the ion-exchange method are characterized by an average size in the range of $1.2-5 \mathrm{~nm}$. Deposits are uniformly distributed over the surface, resulting in a very high loading density. An increase in the amount of deposited metal resulted predominantly in a higher nanoparticle loading density, without any growth in the particle size. Various metal catalysts deposited on the surface of titanate nanotubes are shown in Fig. 24. The deposition method was ion-exchange followed by chemical treatment. Not all catalysts can be deposited using this method. For example, the approach fails in cases where a suitable cationic form of the metal is unavailable in aqueous solution, etc.

An increase in platinum loading results in an increase of the metal particle size. Metal particles formed inside the cavities of titanate nanotubes have a rod-like shape, with the diameter of the rods corresponding to the internal diameter of nanotubes [259].

The Au nanoparticles were produced with a spheroidal shape and with a wide particle size distribution. In the sample with $2 \mathrm{wt} \%$ gold loading the average particle size is ca. $4 \mathrm{~nm}$, much larger than the internal diameter of the nanotubes also present in the sample. This clearly indicates that the deposition of gold nanoparticles occurs on the external surface of nanotubes. An increase in gold loading results in the appearance of rod-like gold particles, with the diameter equal to the internal diameter of the tubes, proving that deposition of gold also occurs inside the titanate nanotubes. Despite blocking of the external surface of nanotubes during reduction with 
Table 6

Textural characteristics and surface acidic sites normalized by the specific area of the catalysts at $200{ }^{\circ} \mathrm{C}$ and $400{ }^{\circ} \mathrm{C}$. Reproduced from Ref. [257].

\begin{tabular}{|c|c|c|c|c|c|c|c|}
\hline Catalyst & $S_{\text {bet }}\left(\mathrm{m}^{2} / \mathrm{g}\right)$ & $D_{\mathbf{p}}(\AA)$ & $V_{\mathrm{p}}\left(\mathrm{cm}^{3} / \mathrm{g}\right)$ & $C_{\text {Lewis }}\left(\mu-\mathrm{mol} \mathrm{m}^{-2}\right)$ & $C_{\text {Brönsted }}\left(\mu-\mathrm{mol} \mathrm{m}^{-2}\right)$ & $C_{\text {Total }}\left(\mu-\mathrm{mol} \mathrm{m}^{-2}\right)$ & Temp. $\left({ }^{\circ} \mathrm{C}\right)$ \\
\hline TAN & 335 & 56 & 0.45 & $\begin{array}{l}0.74 \\
0.01\end{array}$ & $\begin{array}{l}0.19 \\
0.00\end{array}$ & $\begin{array}{l}0.93 \\
0.01\end{array}$ & $\begin{array}{l}200 \\
400\end{array}$ \\
\hline V10/TAN & 329 & 44 & 0.36 & $\begin{array}{l}0.81 \\
0.11\end{array}$ & $\begin{array}{l}0.23 \\
0.00\end{array}$ & $\begin{array}{l}1.04 \\
0.11\end{array}$ & $\begin{array}{l}200 \\
400\end{array}$ \\
\hline $\mathrm{V} 5-\mathrm{W} 3 / \mathrm{TiO}_{2}$ & 55 & 237 & 0.33 & $\begin{array}{l}0.97 \\
0.10\end{array}$ & $\begin{array}{l}0.03 \\
0.00\end{array}$ & $\begin{array}{l}1.00 \\
0.10\end{array}$ & $\begin{array}{l}200 \\
400\end{array}$ \\
\hline
\end{tabular}

$\mathrm{NaBH}_{4}$, gold nanoparticles coat both the internal and external surfaces of nanotubes [259].

TEM images show that palladium could occupy both internal and external surfaces of titanate nanotbes. The metal was deposited by ion-exchange from $\mathrm{PdCl}_{2}$ solution followed by reduction with $\mathrm{NaBH}_{4}$. The average diameter of metal particles is ca. $2-4 \mathrm{~nm}$ and the particles have a spheroidal shape. The particles can be well dispersed on either surface of nanotubes [259].

The TEM images and histograms of hydrated ruthenium (III)-oxide nanoparticles deposited on the surface of titanate nanotubes in three different loadings are shown in Fig. 25. Spherodial ruthenium nanoparticles were evenly distributed on both the internal and the external surface nanotubes [259]. An increase in ruthenium loading from 0.6 to $8.7 \mathrm{wt} \%$ results in an increase in the density of deposited particles, whereas the average size of the particles remains constant.

The interaction mechanism and local atomic structures of $\mathrm{Ni}$ (II) on titanate nanotubes (TNTs) as a function of contact time, solution $\mathrm{pH}$ and addition of humic acid (HA) or fulvic acid (FA) were studied mainly by extended X-ray absorption fine structure spectroscopy (EXAFS). The results indicated that $\mathrm{Ni}$ (II) can be interacted with TNTs by different mechanisms and the actual choice is largely determined by environmental conditions. At $\mathrm{pH} 4.0$ and short contact time, the interaction of $\mathrm{Ni}$ (II) with TNTs is mainly dominated by outer-sphere surface complexation. At medium to increasing $\mathrm{pH}$ the $\mathrm{Ni}$ (II)TNT interaction tends to transform from outer-sphere surface complexes to inner-sphere surface complexes, while a mixture of inner-sphere surface complexes and surface precipitates is formed between $\mathrm{pH} 7.0$ and 8.0. Besides, the interaction of $\mathrm{Ni}$ (II) with TNTs becomes more favorable with time, and EXAFS spectral analysis indicates that at short contact time outer-sphere surface complexes are transformed into innersphere surface complexes and surface precipitates over long contact time. The addition of HA or FA improves Ni(II) interaction with TNTs at low $\mathrm{pH}$, which is attributed to the formation of "metal-bridging" (type A) and "ligand-bridging" (type B) ternary surface complexes. The results obtained are very important for understanding the interaction of $\mathrm{Ni}$ (II) and related heavy metal ions with nano-materials such as TNTs once released into natural environment [260].

The adsorption of $\mathrm{Pb}^{2+}, \mathrm{Cd}^{2+}, \mathrm{Cu}^{2+}$ and $\mathrm{Cr}^{3+}$ from aqueous solution onto titanate nanotubes (TiONT) in multiple systems was studied systematically [261]. Particular attention was paid to competitive adsorption and the effects of inorganic ions. TiONTs exhibited large ion exchange adsorption capacity for heavy metals. Binary or quaternary competitive adsorption indicated that the adsorption capacity of the four heavy metals onto TiONT followed the sequence of $\mathrm{Pb}^{+}{ }_{\gg} \mathrm{Cd}^{+}>\mathrm{Cu}^{+}{ }_{\gg} \mathrm{Cr}^{3+}$ which again followed the reverse order of their hydration energies. Moreover, inorganic ions including $\mathrm{Na}^{+}, \mathrm{K}^{+}, \mathrm{Mg}^{+}$ and $\mathrm{Ca}^{+}$inhibited the adsorption of heavy metals on TiONTs, because they competed for the same adsorption sites, decreased the activity of heavy metal ions, and promoted the aggregation of TiONT. However, $\mathrm{Al}^{3+}$ and $\mathrm{Fe}^{3+}$ generally enhanced adsorption because the resulting hydroxyl-Al/Fe intercalated or coated TiONT could also capture metal ions. The performed study hinted the potential applicability of TiONTs in wastewater treatment for heavy metals. Fig. 26 shows a schematic of heavy metal adsorption onto titanate nanotubes in multiple systems.

Fig. 26(A) illustrates the layered trititanate structure of TNTs and the adsorption possibilities of heavy metal ions [228]. Moreover, the effect of inorganic ions included (Fig. 26 (B)): (1) competition for adsorption sites, (2) reduced activities of metal ions and (3) enhanced aggregation and larger specific surface area of TNTs by electrolyte ions through electric double layer compression. The coated compounds could adsorb even further metal ions (Fig. 26(C)) [228].

Characterization and catalytic performance assessment of cations $\left(\mathrm{M}=\mathrm{Ce}^{4+}, \mathrm{La}^{3+}, \mathrm{Co}^{2+}, \mathrm{Cu}^{2+}\right.$ or $\left.\mathrm{Na}^{+}\right)$intercalated titanate nanotubes (MTNT) were performed for $\alpha, \beta$-unsaturated esters production. The structural properties of the solids were evaluated by transmission electron microscopy (TEM), X-ray diffraction (XRD), and Raman spectroscopy measurements, whereas textural properties were obtained from nitrogen adsorption-desorption isotherms. The transition metals were intercalated in titanate nanotubes by ionic exchange reaction 
A
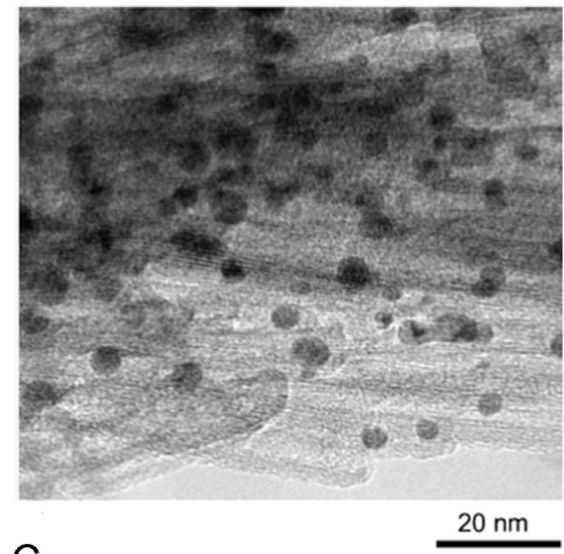

C
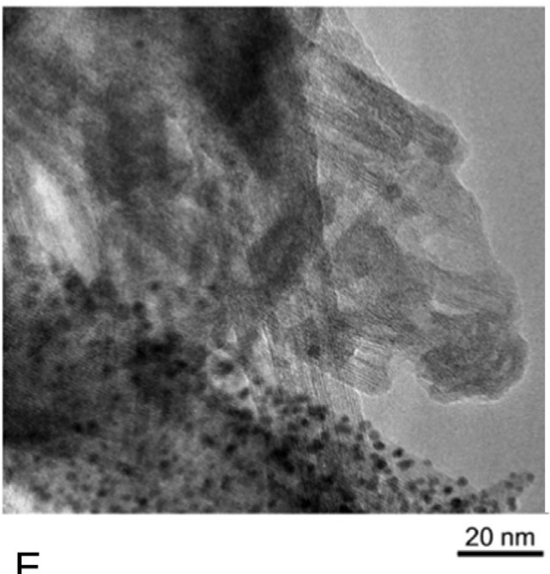

E

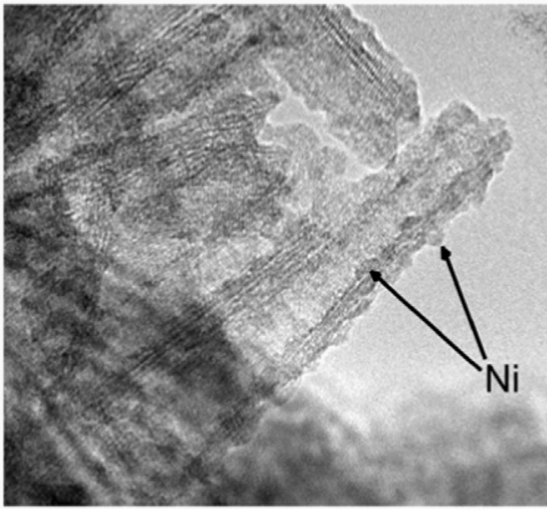

$10 \mathrm{~nm}$
B

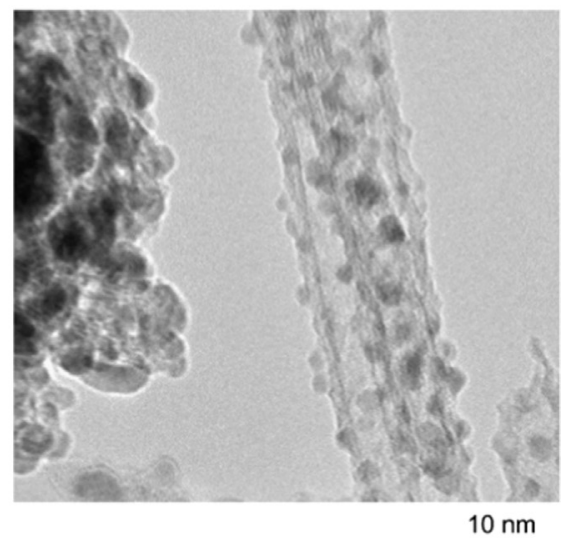

D
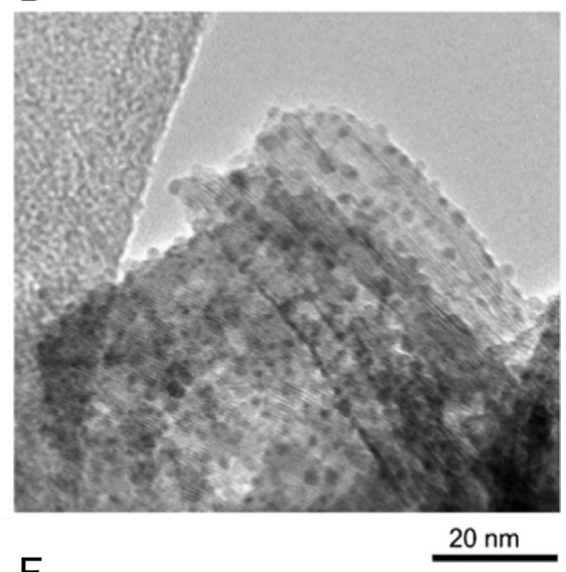

$\mathrm{F}$

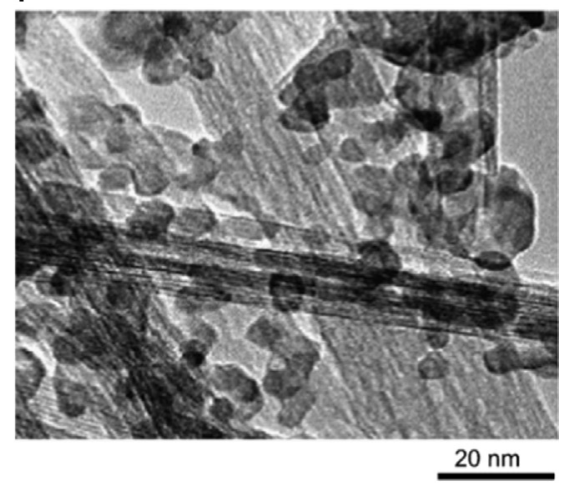

Fig. 24. Titanate nanotubes decorated with metal nanoparticles: (a) Au/TiONT; (f) $\mathrm{CdS} / \mathrm{TiONT}$. Reproduced from Ref. [32].

where the $\mathrm{Na}^{+}$cations were substituted by $\mathrm{Ce}^{4+}, \mathrm{La}^{3+}, \mathrm{Co}^{2+}$ and $\mathrm{Cu}^{2+}$. The structural characterizations revealed that the ion-exchange processes can favor the formation of nanosized oxides on the TNT surfaces, in some cases [262].

A two-step method was developed for the synthesis of onedimensional lead titanate $\left(\mathrm{PbTi}_{3} \mathrm{O}_{7}\right)$ nanotubes. Firstly, titanate nanotubes were prepared by an alkaline hydrothermal process from $\mathrm{TiO}_{2}$ nanopowder precursor, then lead titanate nanotubes were formed by ion exchange. It was found that sodium ions in titanate nanotubes are exchangeable with lead ions, while protons are not. For the first time, a difference was observed between sodium titanate nanotubes and protonated titanate nanotubes in the ion-exchange process, which reveals a layer space effect of nanotubes in the ion-exchange reaction. In comparison with sodium titanate, the synthesized lead titanate nanotubes show a narrowed bandgap [263].

In a related system a robust, low-cost method to attach transition metal ions directly to the surface of anatase $\mathrm{TiO}_{2}$ rodshape nanocrystal morphology and phases was reported [264]. $\mathrm{TiO}_{2}$ nanorods were synthesized in gram-scale quantities according to the procedure reported by Hyeon [265]. The oleic acid stabilized anatase $\mathrm{TiO}_{2}$ nanorods dispersed readily in a 

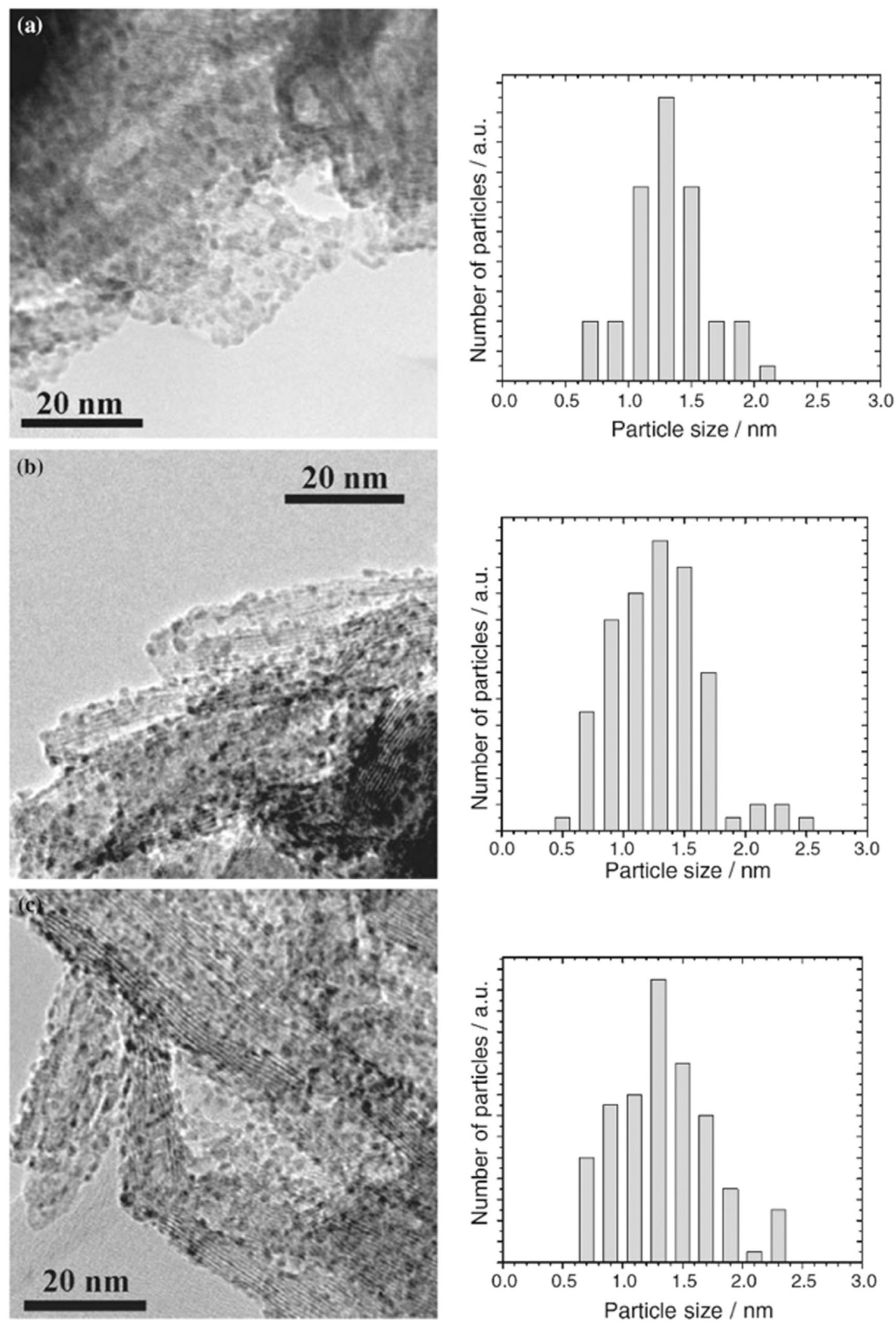

Fig. 25. TEM images of hydrated ruthenium(III)-oxide nanoparticles deposited onto titanate nanotubes and histograms of particle size distribution for different Ru loadings: (a) $1.1 \mathrm{wt} \%$, (b) $3.4 \mathrm{wt} \%$, (c) $8.7 \mathrm{wt} \%$. Reproduced from Ref. [259].

non-polar solvent and were characterized by powder X-ray diffraction, TEM and UV-visible spectroscopy. Unprecedented control was demonstrated over the attachment of transitional metal ions to the surface of well-defined inorganic nanocrystals in non-polar solvent analogous to solution atomic layer deposition. The method uses inexpensive metal chloride hydrates and 

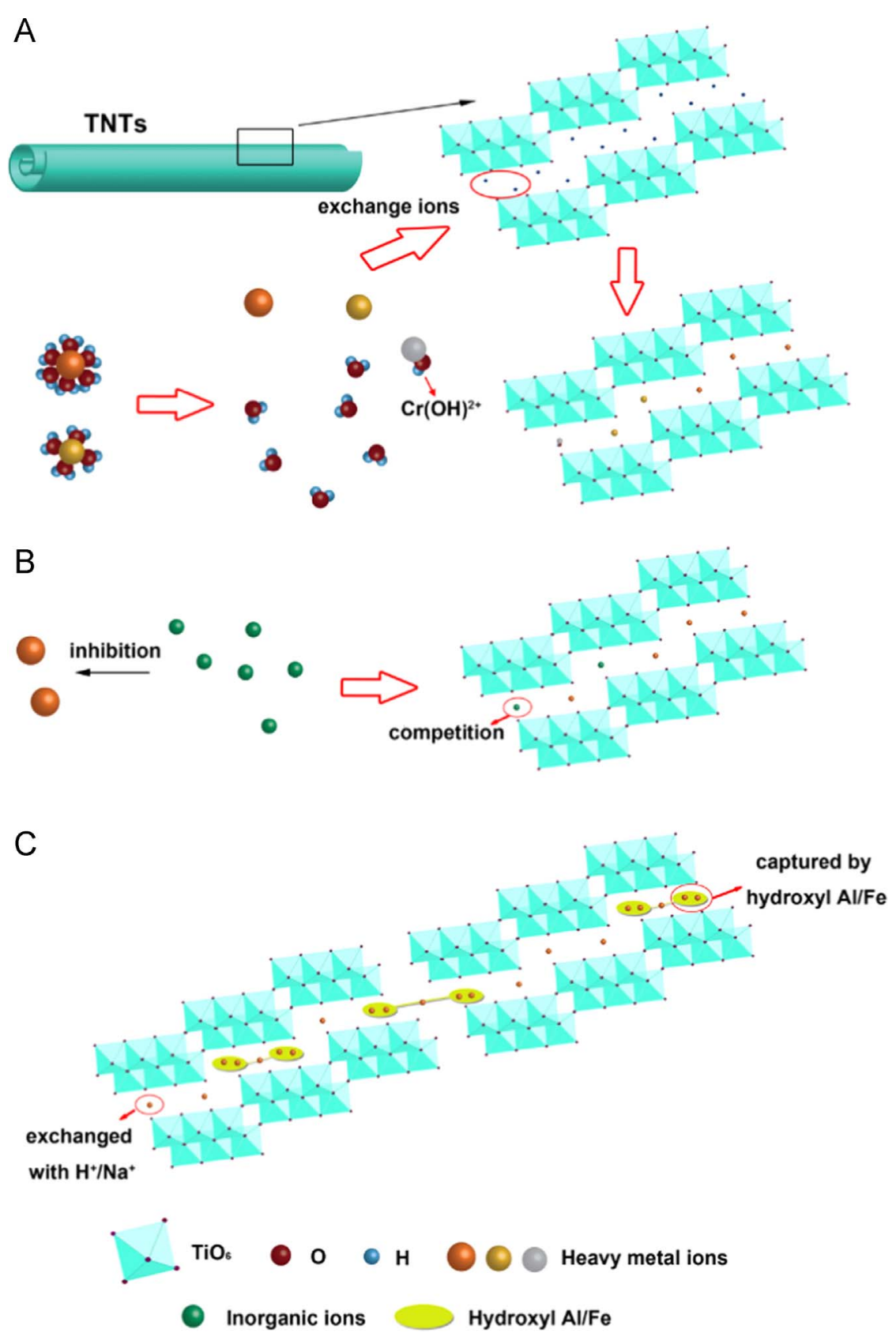

Fig. 26. Schematic of heavy metal adsorption onto TNTs. Reproduced from Ref. [228].

allows variable loading of the nanocrystal surface with $\mathrm{Cr}^{3+}$, $\mathrm{Mn}^{2+}, \mathrm{Fe}^{3+}, \mathrm{Co}^{2+}, \mathrm{Ni}^{2+}$ and $\mathrm{Cu}^{2+}$ to the point of saturation.

Crystalline Fe-doped trititanate nanotubes were synthetized by a wet-chemical method [266]. The products were characterized by high-resolution transmission electron microscopy (TEM), energy-dispersive X-ray spectrometry (EDS), in-situ $\mathrm{X}$-ray diffraction (XRD), ultraviolet-visible spectrophotometry (UV-vis), synchrotron infrared (IR) spectroscopy, and temperature-dependent magnetometry. The Fe-doped trititanate nanotubes were about $8 \mathrm{~nm}$ in diameter and up to $1 \mu \mathrm{m}$ long. EDS revealed that the Fe-doping ratio is about $0.5 \%$, while the effects of doping were uncovered by IR and UV-vis spectra.
The optical band gap of the doped nanotubes was $3.05 \mathrm{eV}$, which is smaller than that of undoped trititanate nanotubes $(3.4 \mathrm{eV})$.

It has been reported that the ion exchange reactions performed with TiONTs lead to the intercalation of $\mathrm{Ce}^{4+}$ and $\mathrm{Ce}^{3+}$ (produced via reduction reactions in the aqueous solution) ions into the titanate nanotubes walls. Above a critical concentration of cerium ion in the aqueous solution, the decoration of the outer nanotube surfaces with $\mathrm{CeO}_{2}$ nanoparticles takes place via hydrolysis reaction in the aqueous solutions and the attachment of the nanoparticles [267]. It was shown that the ion exchange reaction preserved the atomic 
structure of the TiONT, but introduced changes in its interlayer environment. EDS measurements revealed that the cerium content in the small nanoparticles found on the surface of the nanotubes is higher than the cerium amount intercalated into the nanotubes.

Titania nanotubes can also be prepared by electrochemical method. They are similar to titanates, however, without cations in ion exchange positions. Therefore, metals can form only particles of different sizes on their surface. We demonstrate one example for this metal containing structure. Recently $\mathrm{Pd}$ nanoparticles were produced and investigated on a selforganized $\mathrm{TiO}_{2}$ nanotubular array [268]. The Pd nanoparticles were prepared by two techniques, i.e. physical vapor deposition (PVD) in ultrahigh vacuum (UHV) and by particle precipitation (PP) from solution. The particle size could be varied over a broad range by both methods. Whereas PVD leads to a system with Pd particles located close to the orifice of the tubes, a homogeneous distribution of particles inside the tubes can be obtained for Pd deposition via PP. The surface of the Pd nanoparticles was characterized by $\mathrm{CO}$ adsorption (Fig. 27).

So far we focused on the preparation of metal-doped titanate nanowires and nanotubes. They were thoroughly characterized by morphology and structure sensitive methods. However, in many cases attempts to investigate the interaction of the metal with titanate surface in atomic level were missing. In the next paragraph we characterize such interactions by photon-, ion and electronspectroscopic methods and simultaneously monitor morphological and structural changes as well.

\subsubsection{Formation and stabilization of $\mathrm{Co}^{2+}$ and Co particles} on titanate nanostructures. The study of fabrication and properties of size-controlled Co nanoparticles supported on oxide supports is an important task from the catalytic point of view. Cobalt-based catalysts are widely used in the reaction of $\mathrm{CO}$ and $\mathrm{H}_{2}$ to form linear aliphatic hydrocarbons with a broad molecular weight distribution [269]. Recently, cobalt (which is less expensive than noble metals) was found to be an excellent catalyst for ethanol transformation to produce hydrogen as an alternative energy carrier [270,271]. The low temperature decomposition of metal carbonyls is an elegant way to produce metal nanoparticles on substrates [272-274] (comparable similar methods are photo-induced [275] and electronassisted [276-279] metal-CO bond cleavage in carbonyl compounds). The main advantage of low temperature carbonyl decomposition is that no high temperature calcination is necessary, thus morphological changes and metal particle agglomeration can be avoided. Moreover, surface $\mathrm{Co}_{2} \mathrm{O}_{3}$ formation can also be prevented this way, which is important because this species cannot be reduced completely even at high temperatures (800-1000 K).

The surface deposition of dicobalt octacarbonyl $\left(\mathrm{Co}_{2}(\mathrm{CO})_{8}\right)$ was carried out on $\mathrm{H}$-form titanate nanowires in a fluidized bed reactor designed for controlled experiments at low temperatures $[280,281] . \mathrm{Co}_{2}(\mathrm{CO})_{8}$ was supplied by Fluka Chemie AG and was used without further purification. Owing to its sensitivity to air it was stored under $\mathrm{CO}$ and moved into the sublimation chamber through a glovebox. Before the carbonylation process the $\mathrm{H}$-form titanate nanowires were thermally treated for $1 \mathrm{~h}$ in nitrogen at $773 \mathrm{~K}$. XPS and FTIR analysis evidenced that the weakly adsorbed water and $\mathrm{OH}$ groups were removed during the heat treatment. The ordered $\mathrm{TiO}_{2}$ anatase structure starts to develop at this temperature $[35,92,282]$. The detailed physico-chemical characterization of cobalt loaded titanate nanowires will be reported below in the following order: (i) $\mathrm{Co}_{2}(\mathrm{CO})_{8}$ decomposition, (ii) titanate optical properties, (iii) detailed XPS characterization of the system and (iv) product morphology.

Cobalt carbonyl adsorbed on protonated titanate nanowires was characterized by FT-IR spectroscopy. In the solid state the structure of $\mathrm{Co}_{2}(\mathrm{CO})_{8}$ is of $\mathrm{C}_{2 \mathrm{v}}$ point group symmetry involving a pair of bridging $\mathrm{CO}$ groups. The IR spectrum of adsorbed carbonyl at $323 \mathrm{~K}$ is displayed in Fig. 28. This spectrum is very similar to that observed in heptane solution [283] and it is close to that obtained on porous silica [284]. The observed deviations can be attributed to differences in the substrate effect and the different degree of the isomerization process. Two strong bands were observed at 1986 and $2036 \mathrm{~cm}^{-1}$ and weak and broad bands were detected at 1928 and $1872 \mathrm{~cm}^{-1}$, respectively [280]. When the carbonyl-containing surface was heated to higher temperatures, all bands decreased in intensity. The characteristic bands disappeared at about $623 \mathrm{~K}$ indicating the completion of the $\mathrm{Co}_{2}(\mathrm{CO})_{8}$ decomposition process.

The optical properties of cobalt-decorated titanate nanowires were studied by diffuse reflectance UV-vis spectra. The changes in the band gap of titanate nanowires after the decomposition of cobalt carbonyl were determined. The color of the samples changed from yellowish to intense yellow with increasing carbonylation time. Correspondingly, the absorption edge shifted into the visible range. The band gap energy $\left(E_{g}\right)$ was calculated according to Beranek and Kisch, from the plots of $\left[F\left(R_{\infty}\right) / h \nu\right]^{1 / 2}$ vs. $\mathrm{h} \nu$ [218]. The band gap for pure titanate nanowire was $3.10 \mathrm{eV}$, while that for Co-doped titanates was significantly less, 2.48 for $2 \mathrm{wt} \%$ and $2.41 \mathrm{eV}$ for $4 \mathrm{wt} \%$ Co content. No significant changes were observed above $2 \mathrm{wt} \%$ metal loading [280].

In a reference experiment the band gap of high surface area commercial $\mathrm{TiO}_{2}$ (Degussa P25) decreased to $3.03 \mathrm{eV}$ when depositing $2 \mathrm{wt} \%$ cobalt onto it by low termperature $\mathrm{Co}_{2}(\mathrm{CO})_{8}$ decomposition. The more pronounced decrease of the band gap of titanate nanowires upon loading with $2 \mathrm{wt} \%$ Co suggests a very strong electronic interaction between the titanate nanowire framework and cobalt, which may eventually result in an ion exchange process similar to that occurring in silver loaded titanate nanotubes (see below) [233].

Detailed XPS measurements were performed on the $4 \mathrm{wt} \%$ cobalt containing sample (Fig. 29).

In the "as received sample" the $2 p_{3 / 2}$ photoemission appeared at $781.7 \mathrm{eV}$, the $2 \mathrm{p}_{1 / 2}$ emission was located at $797.5 \mathrm{eV}$ and strong satellite structures were also present for $2 p_{3 / 2}$ and $2 p_{1 / 2}$, respectively (Fig. 29(A)). The spin orbit coupling $(15.8 \mathrm{eV})$ is almost the same as for $\mathrm{Co}^{2+}$ and a little bit higher than for metallic Co $[273,285,286]$.

Two new O 1s XPS peaks appeared in addition to the titanate lattice oxygen signal $(530.1 \mathrm{eV})$ after carbonylation at 

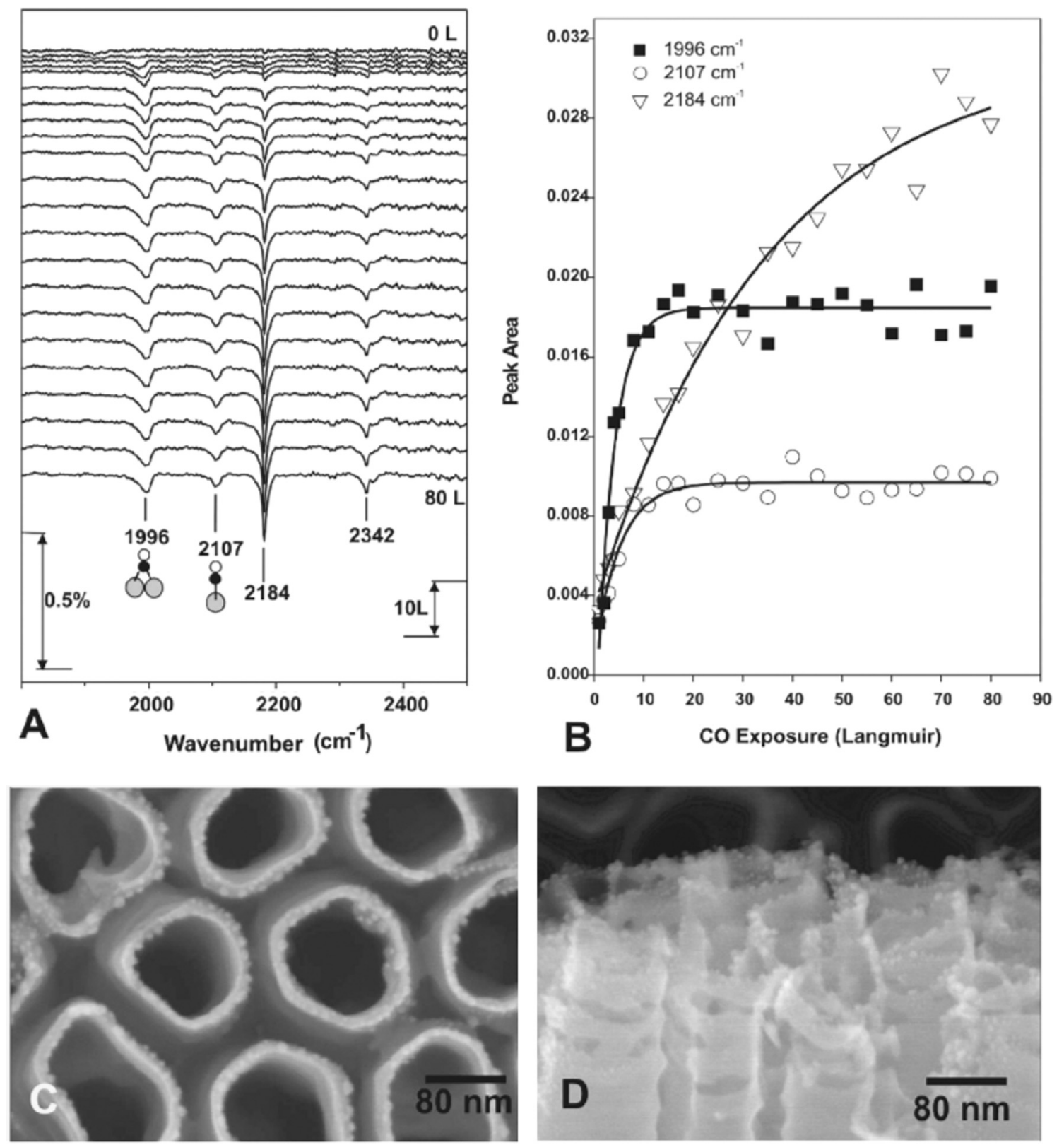

Fig. 27. (A) IRAS spectra of $\mathrm{CO}$ adsorbed on $\mathrm{Pd}\left(\mathrm{PVD}, 0.8 \mathrm{~nm}\right.$ )/TiNT acquired at $90 \mathrm{~K}$ after $\mathrm{UHV}$ deposition of $\mathrm{Pd}$ at room temperature and cleaning with $\mathrm{O}_{2} / \mathrm{CO}$. (B) Evolution of the CO peak area with gas exposure. (C) Top view SEM of the TiNTs decorated with Pd nanoparticles of 4-5 nm average size. (D) SEM image cross-section view showing the nanoparticles present mostly distributed within $\sim 100 \mathrm{~nm}$ of from the top of the TiNTs. Reproduced from Ref. [268].

531.7 and $532.6 \mathrm{eV}$ (Fig. 30(A)). The dominant emission is at $531.7 \mathrm{eV}$, while the peak at $532.6 \mathrm{eV}$ has much less intensity. An intense $\mathrm{C} 1 \mathrm{~s}$ signal showed up due to the carbonyl fragment at $289.6 \mathrm{eV}$ and a weaker one at $288.6 \mathrm{eV}$ (Fig. 31 (A)). These two emissions can be assigned to adsorbed carbonyl fragments in two orientations. When the carbonylated sample was heated to $600 \mathrm{~K}$ at which $\mathrm{CO}_{2}$ is already formed, the intensity of the Co $2 \mathrm{p}$ signal did not decrease but the peak shifted towards lower binding energies by 0.7 eV (Fig. 29(B)). Interestingly, the binding energy positions of satellites remained. At this temperature the intensities of the $\mathrm{O} 1 \mathrm{~s}$ peaks representing different orientations and/or different carbonyls $\left(\mathrm{Co}_{2}(\mathrm{CO})_{8}, \mathrm{Co}_{4}(\mathrm{CO})_{12}\right)$ at 531.7 and $532.6 \mathrm{eV}$ for $\mathrm{O} 1 \mathrm{~s}$ diminished or decreased significantly (Fig. 30(B)). The remaining small intensity emissions could be assigned either to undecomposed carbonyls or "bridging $\mathrm{O}$ " and "top $\mathrm{O}$ " groups on the titanate [198]. The O 1s feature did not change upon oxidation (Fig. 30(C)) or further reduction (Fig. 30(D)) of the sample at $600 \mathrm{~K}$ for $60 \mathrm{~min}$. Similarly to the O $1 \mathrm{~s}$ photoemissions, the intensity of $\mathrm{C} 1 \mathrm{~s}$ signals for carbonyl species also diminished after decarbonylation (Fig. 31(B)). The observed small intensity $\mathrm{C} 1 \mathrm{~s}$ peaks could be attributed to the still intact carbonyls and/or different carbon species remaining from the decomposition of carbonyl. The peak at $285.0 \mathrm{eV}$ is due to the residual carbon contamination of the titanate nanowires. 


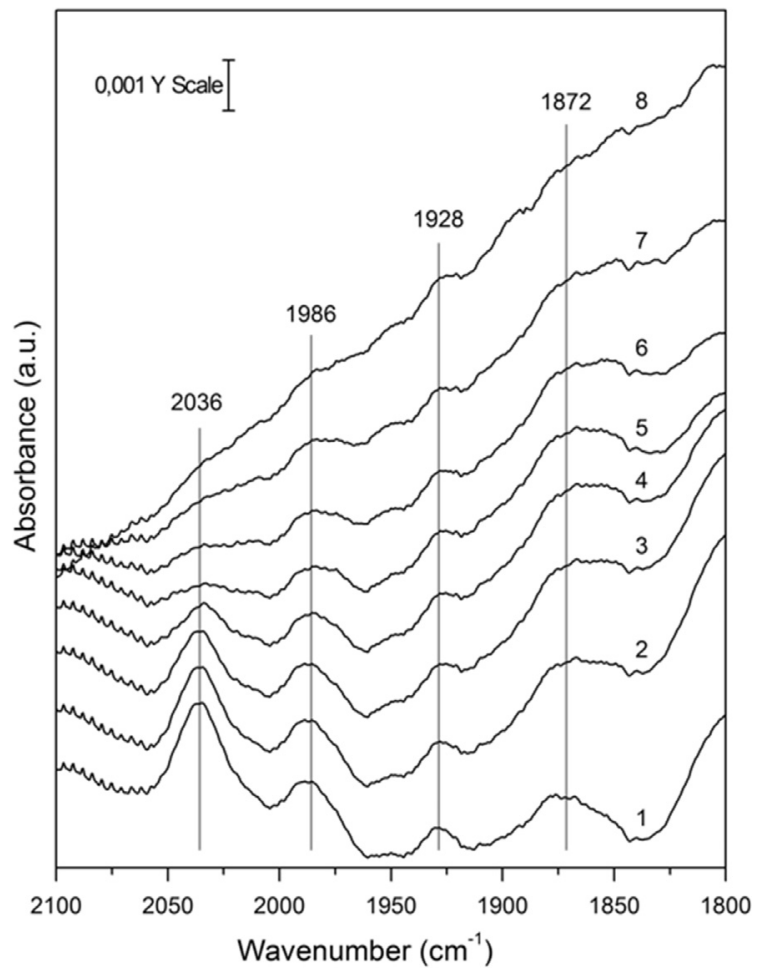

Fig. 28. FTIR spectra of adsorbed $\mathrm{Co}_{2}(\mathrm{CO})_{8}$ on protonated titanat nanowire and heated with $20 \mathrm{~K} / \mathrm{min}$ in $\mathrm{He}$ at different temperatures: (1) $-323 \mathrm{~K}$; (2) $-373 \mathrm{~K}$; (3) $-423 \mathrm{~K}$; (4) $-473 \mathrm{~K}$; (5) $-523 \mathrm{~K}$; (6) $-573 \mathrm{~K}$; (7) $-623 \mathrm{~K}$; and $(8)-673 \mathrm{~K}$. Resolution: $1 \mathrm{~cm}^{-1}$, number of scans: 16 . Spectra were taken at given temperatures. Reproduced from Ref. [280].
The findings discussed above suggest that decarbonylation occurs between 300 and $600 \mathrm{~K}$. The observed Co $2 p$ photoemission peaks are higher than those of pure metallic Co and in addition the "oxide like" satellite structure remained unchanged even though the intensities of the $\mathrm{O} 1 \mathrm{~s}$ peaks at $531.7 \mathrm{eV}$ and $532.6 \mathrm{eV}$ decreased drastically. When the decarbonylated surface was kept in $\mathrm{O}_{2}$ atmosphere at $600 \mathrm{~K}$ for $60 \mathrm{~min}$, the Co $2 \mathrm{p}_{3 / 2}$ peak position moved to lower binding energies to $780.3 \mathrm{eV}$ and its intensity decreased (Fig. 29(C)). These two changes can be attributed to the formation of oxides [285,287] and the agglomeration/encapsulation of clusters [288-290]. When the sample was treated further in $\mathrm{H}_{2}$ atmosphere at $600 \mathrm{~K}$ for $60 \mathrm{~min}$ a small intensity Co $2 \mathrm{p}_{3 / 2}$ peak appeared at $779.6 \mathrm{eV}$ indicating the tendency for the reduction of Co species (Fig. 29(D)) [285,288]. The intensity changes correlate also with the coalescence and diffusion into the bulk.

Due to the preparation method of titanate nanowires (structural water release during heat treatment) and $\mathrm{CO}_{2}$ formation upon carbonyl decomposition the nanowires may contain more defects than reduced commercial titania. Therefore, it may be supposed that after carbonyl decomposition cobalt is found in a strongly oxygen vacant environment in high dispersion and small cluster size (2-5 nm) (Fig. 32). Core level shifts due to the nanoparticle size must be considered in the interpretation of such spectra [291-293]. Initial effects like the intrinsic size effect caused by the reduced average coordination number of surface atoms in small particles lead
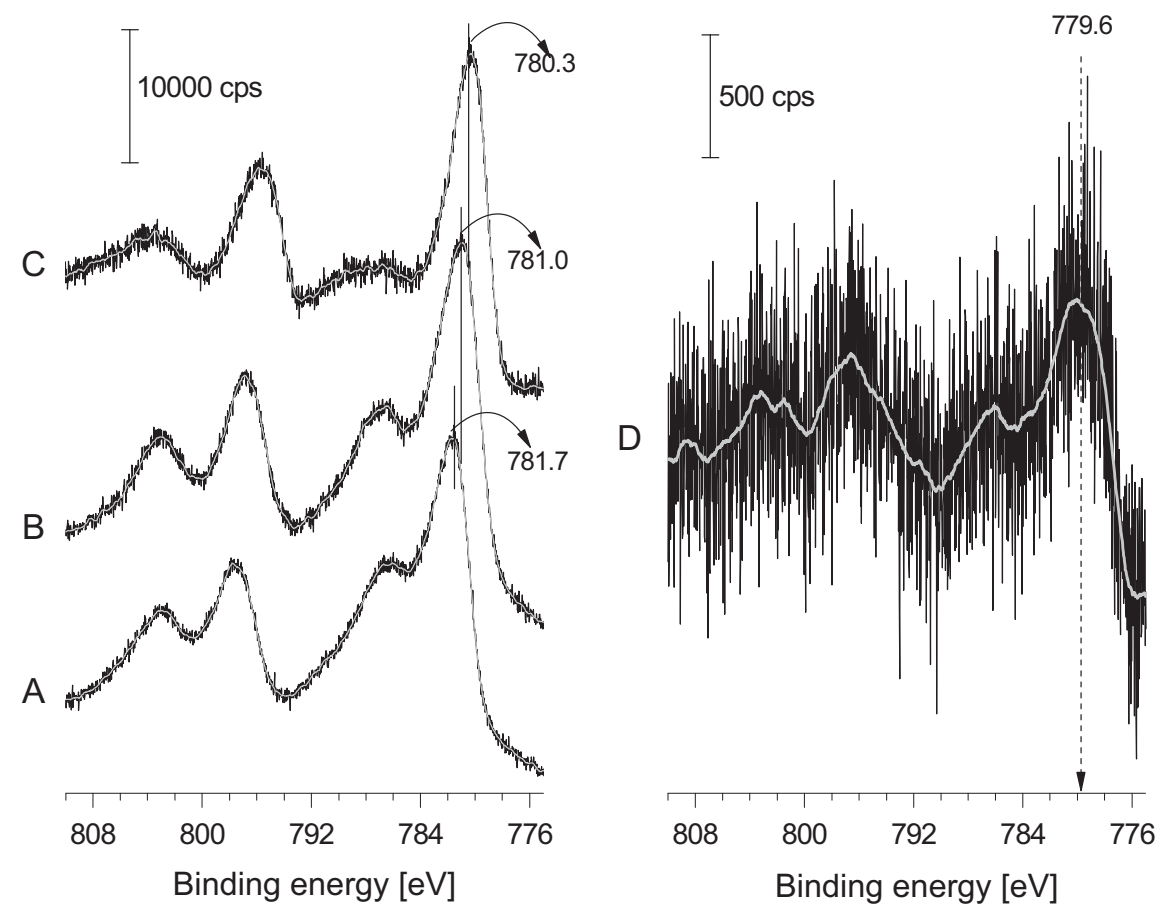

Fig. 29. Co $2 p$ photoemission spectra: (A) as received carbonylated titanate nanowire and (B) decarbonylated sample at $4 \%$ metal content (decomposed sample at $600 \mathrm{~K}$ in $\mathrm{N}_{2}$ atmosphere for $10 \mathrm{~min}$ ). (C) Decarbonylated sample after oxidation at $600 \mathrm{~K}$ for $60 \mathrm{~min}$, and (D) after reduction at $600 \mathrm{~K}$ for 60 min. Reproduced from Ref. [280]. 
A

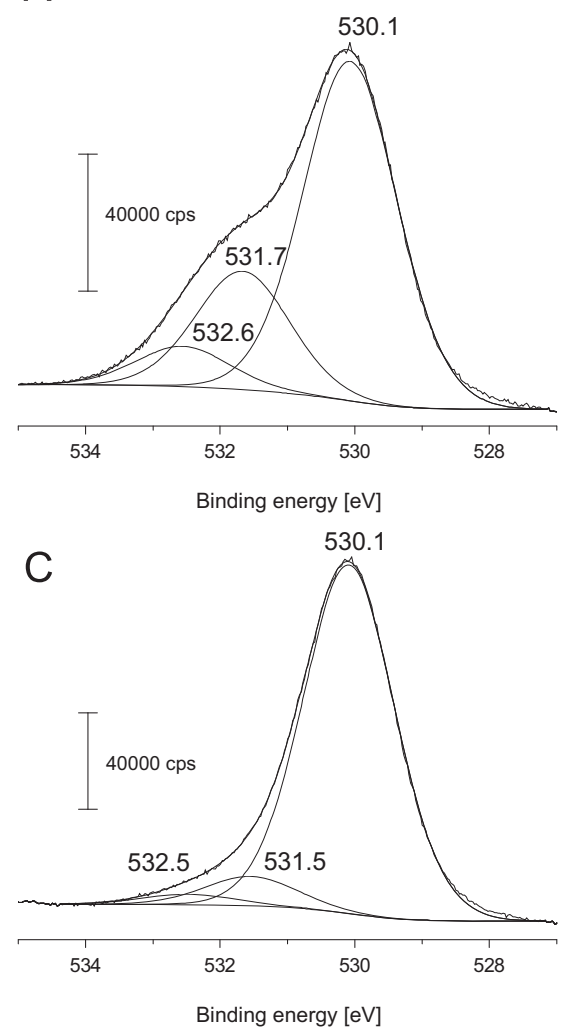

B

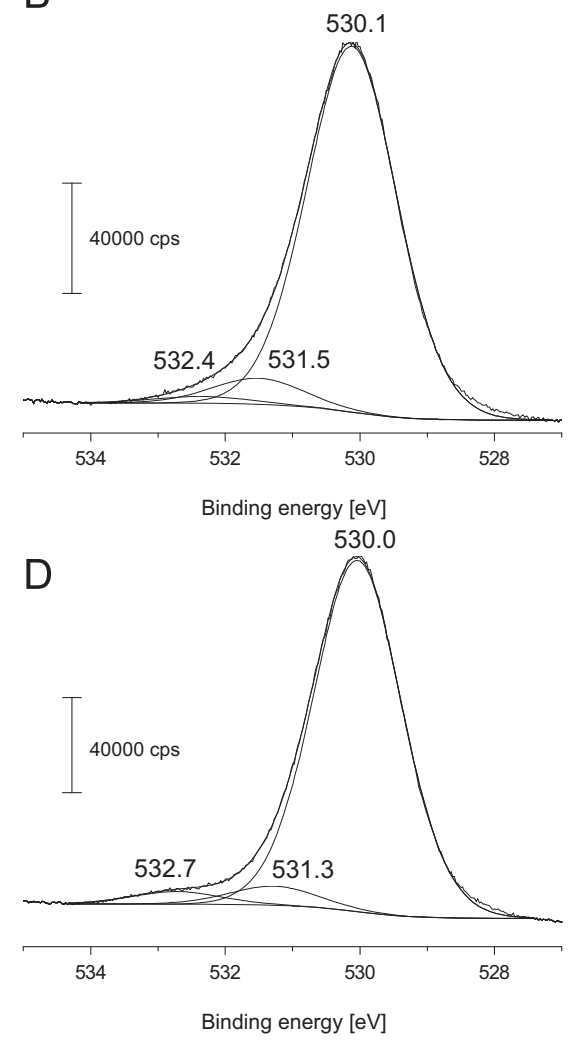

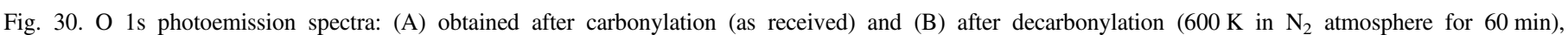
(C) oxidation at $600 \mathrm{~K}$ for $60 \mathrm{~min}$, and (D) reduction at $600 \mathrm{~K}$ for $60 \mathrm{~min}$. Reproduced from Ref. [280].

to decreasing binding energy for core level electrons. Furthermore, electron exchange with the support can change the binding energy of electrons emitted from the nanoparticle. The binding energy is also influenced by relaxation energy. This "final-state" effect depends on particle size, too [292]. The stabilization process of small clusters may also involve an electron transfer step from the cobalt to the titanate nanostructure, which is manifested in a higher binding energy position of Co. When the sample was treated further in $\mathrm{H}_{2}$ atmosphere at $600 \mathrm{~K}$ for $60 \mathrm{~min}$ a small intensity Co $2 \mathrm{p}_{3 / 2}$ peak appeared at $779.6 \mathrm{eV}$ indicating the tendency for the reduction of Co species (Fig. 29(D)). The intensity changes correlate also with the coalescence and diffusion into the bulk. Similar aggregation was observed when the sample was treated at $873 \mathrm{~K}$. This change can be observed also in the distribution of particles sizes (Fig. 33(A) and (B)).

The band gap decreased from $3.14 \mathrm{eV}$ to $2.41 \mathrm{eV}$ with increasing Co content up to $2 \mathrm{wt} \%$ cobalt content. This drastic reduction was not observed when Co was deposited in a similar way on commercial $\mathrm{TiO}_{2}$. The significant change in the band gap due to Co loading suggests that cobalt is stabilized in the structure by ion exchange up to $2 \mathrm{wt} \%$ loading. Co particles were not detectable by HRTEM below $2 \mathrm{wt} \% \mathrm{Co}$, probably due to the ionic form of cobalt. The reduced band gap may be exploited by future photocatalytic applications of Codecorated titanate nanowires.

Higher cobalt loadings $(4 \mathrm{wt} \%)$ lead to the formation of dispersed Co nanoparticles complexed to oxygen vacancies. The shift from ion-exchange to cluster formation stabilization pathway is suggested to be due to the saturation of available surface ion exchange positions in the titanate nanowire. The average Co particle sizes were between 2 and $6 \mathrm{~nm}$, which could be a favorable regime for some important low-temperature catalytic reactions, e.g. ethanol transformation [280].

Though protonated titanate nanotubes (H-TNTs) have a poor exchangeability at $\mathrm{pH} \sim 7$, it was proved that $\mathrm{H}$-TNTs actually exhibit large ion exchange capacity when their surface protons are removed by neutralization reaction $[232] .\left[\mathrm{Co}\left(\mathrm{NH}_{3}\right)_{6}\right]^{2+}$ cations can be ion exchanged into titanate nanotubes to a large extent to neutralize the negative charge of nanotube multilayers and maintain charge balance. The ion exchange of $\mathrm{H}$ TNTs with $\left[\mathrm{Co}\left(\mathrm{NH}_{3}\right)_{6}\right]^{2+}$ under $\mathrm{pH}>10$ causes the walls of titanate nanotubes to loosen up. The d-value of interlayer space is slightly larger for ion exchanged titante nanotubes (CoTNTs) than for H-TNTs. Thus, the experiment suggested that the ion exchange capacity of protonated titanate nanotubes can be changed significantly by deprotonation in alkali environment $(\mathrm{pH}>10)$.

The band gap of protonated titanate nanotubes $(3.2 \mathrm{eV})$ is narrowed after ion exchange. The band gap of Co-TNTs (5 wt\%) is about $2.4 \mathrm{eV}$. With higher cobalt loading their band gap becomes smaller. For all the Co-TNTs samples, there is a band gap maximum centered around $2.0 \pm 0.1 \mathrm{eV}$, corresponding to excitation at $600 \mathrm{~nm}$ [294].

Co-doped titanate nanotubes were synthesized through the $430 \mathrm{~K}$ treatment of Co-doped $\mathrm{TiO}_{2}$ powders in high 
A

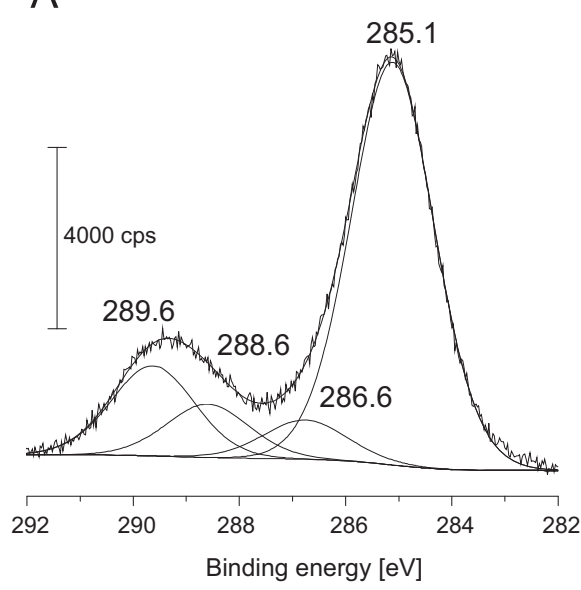

C

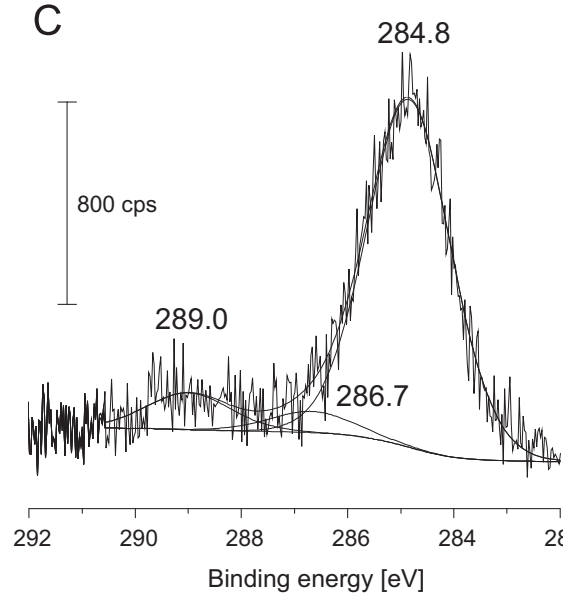

B

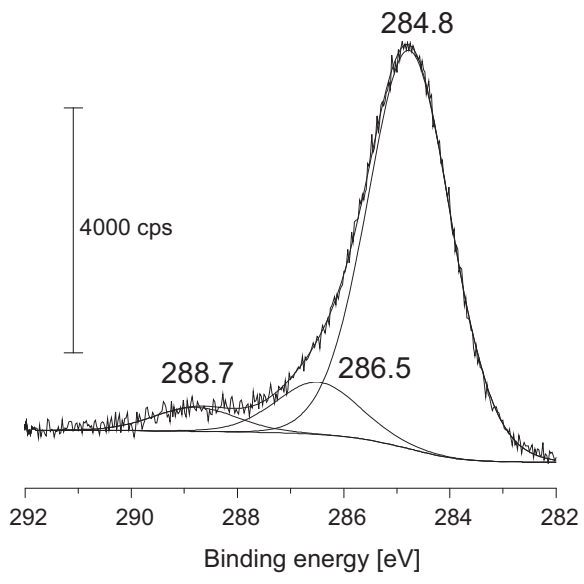

D

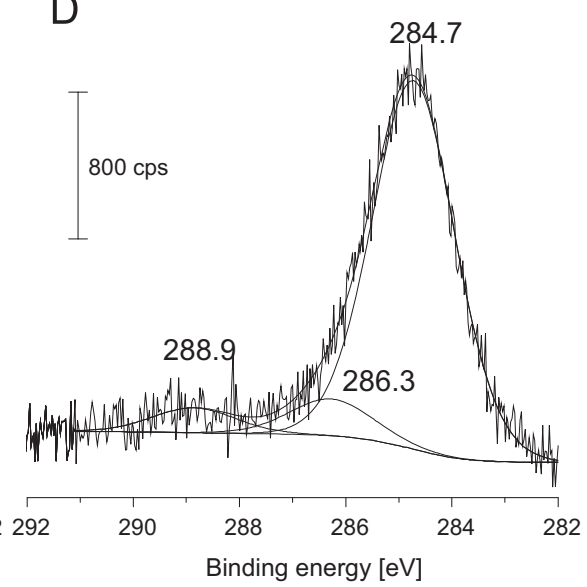

Fig. 31. C 1s photoemission spectra: (A) obtained after carbonylation (as received) and (B) after decarbonylation $\left(600 \mathrm{~K}\right.$ in $\mathrm{N}_{2}$ atmosphere for 60 min), (C) oxidation at $600 \mathrm{~K}$ for $60 \mathrm{~min}$, and (D) reduction at $600 \mathrm{~K}$ for $60 \mathrm{~min}$. Reproduced from Ref. [280].
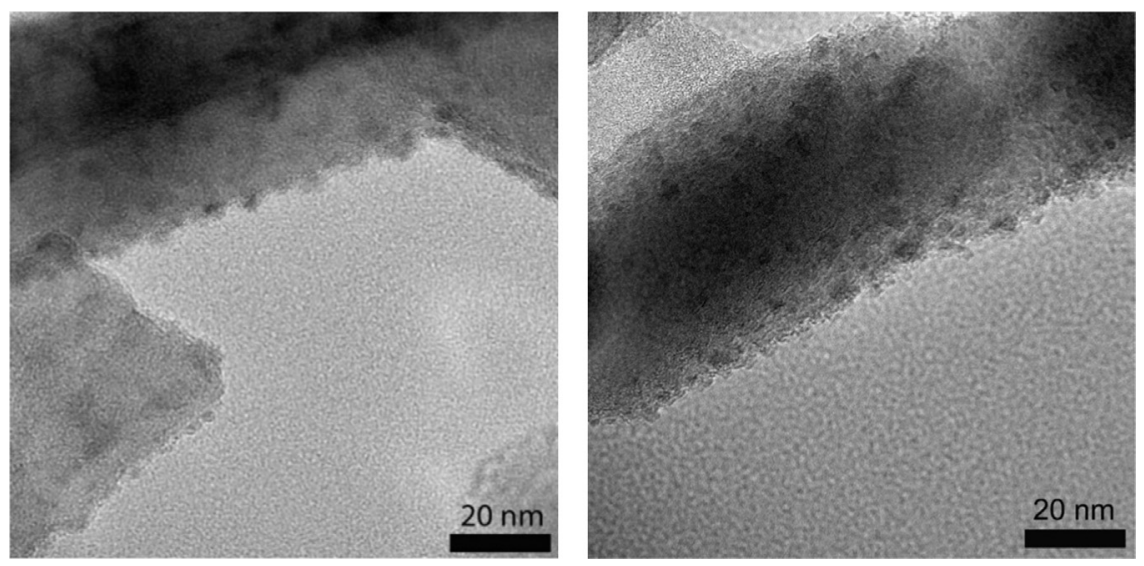

Fig. 32. TEM images of protonated titanate nanowire treated at different temperatures for $60 \mathrm{~min}$ in air (left), after decomposition of $\mathrm{Co}_{2}(\mathrm{CO})_{8}$ on $\mathrm{H}$-form titanate nanowire (right) and after flashing the $\mathrm{Co}_{2}(\mathrm{CO})_{8}$ covered sample up to $873 \mathrm{~K}$. The metal content was 4 wt\%. Reproduced from Ref. [280].

concentration $\mathrm{NaOH}$ aqueous solutions in a hydrothermal autoclave. The obtained nanotubes were identified to be of a monoclinic layered trititanate structure, the same as $\mathrm{H}_{2} \mathrm{Ti}_{3} \mathrm{O}_{7}$, their un-doped counterpart. The incorporation of Co ions was identified by energy dispersive spectroscopy. The Ti:Co atomic ratio in the obtained nanotubes was found to be significantly less than the value in the powder precursor. This indicates that the precursor powders were deformed in $\mathrm{NaOH}$ solution before the formation of nanotubes [295].

Millimeter-sized cobalt doped rutile crystals with a bipyramidal shape are obtained by chemical vapor transport using scroll-type $\mathrm{H}_{2} \mathrm{Ti}_{3} \mathrm{O}_{7}$ nanotubes as a precursor in which $\mathrm{Co}^{2+}$ ions are introduced by a simple ion exchange method prior to the growth [296]. Despite the low concentration of 

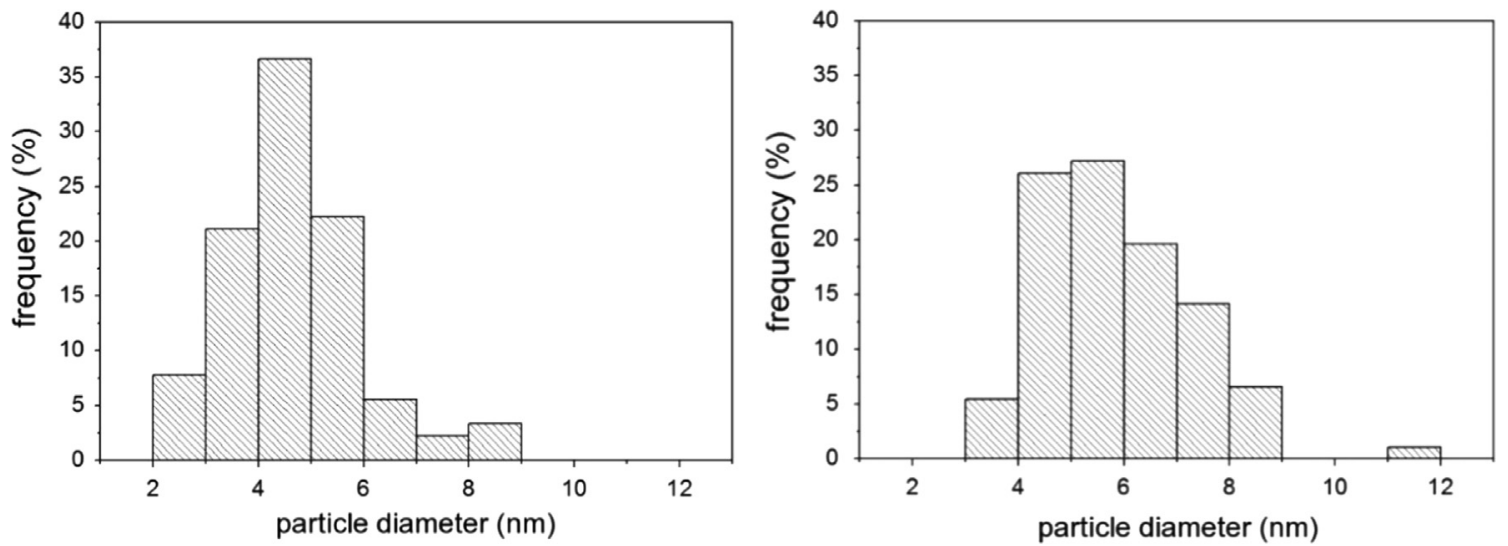

Fig. 33. Diameter distribution of Co nanoarticles (left) obtained after decomposition of $\mathrm{Co}_{2}(\mathrm{CO})_{8}$ on protonated titanate nanowire $\left(600 \mathrm{~K}\right.$ in $\mathrm{N}_{2}$ atmosphere for $60 \mathrm{~min})$, and (right) after flashing the $\mathrm{Co}_{2}(\mathrm{CO})_{8}$ covered sample up to $873 \mathrm{~K}$. The samples were heated with a heating rate of $10 \mathrm{~K} \mathrm{~min}{ }^{-1}$ in $\mathrm{He} 4.6 \mathrm{stream}(40 \mathrm{ml} /$ min). Reproduced from Ref. [280].

$\mathrm{Co}^{2+}$ dopants $\left(5 \times 10^{19} \mathrm{~cm}^{-3}\right)$, the resistivity of the single crystal shows a metallic behavior above $50 \mathrm{~K}$ and the Seebeck coefficient has the signatures of polaronic quasiparticles. The magnetic properties of the material show a weak antiferromagnetic interaction between the spins on Co atoms below $50 \mathrm{~K}$. This synthesis method could be beneficial for the growth of a large variety of doped $\mathrm{TiO}_{2}$ single crystals.

These experimental data (taken from five different laboratories using different synthesis methods) prove that Co cations can be ion exchanged into titanate nanotubes to a large extent to neutralize the negative charge of nanotube multilayers and maintain charge balance. The obtained new nanostructures are promising for catalysis and for producing anti-ferromagnetic materials.

\subsubsection{Surface characterization of silver loaded titanate} nanotubes. In particular, titanate nanotubes (TiONT) combine the properties and applications of $\mathrm{TiO}_{2}$ nanoparticles (e.g., wide band gap semiconductor, photocatalyst) with the properties of layered titanates (e.g. ion exchange) with tubular morphology [297]. Morphology and structure of the titanate precursor and of the formed $\mathrm{TiO}_{2}$ particles (both pure and silver loaded) were investigated by $\mathrm{N}_{2}$ adsorption and power X-ray diffraction analysis, HRTEM, AFM and SEM. Surface and optical properties were explored by FTIR and UV/vis diffuse reflectance spectroscopies. The comparison of FTIR features of adsorbed $\mathrm{CO}$ on pure and silver exchange titanates, with respect to pure and silver loaded $\mathrm{TiO}_{2}$ was reported [233]. H-form titanate powder was ion exchanged with $\mathrm{AgNO}_{3}$ solution at room temperature before filtering, drying and calcining. Then the sample was thermally treated at $773 \mathrm{~K}$. It was concluded in the literature that the thermal treatments affect the morphology of the starting samples [32]. This morphological change may reflect to the surface structure of deposited metals including silver.

Fig. 34 shows the diffuse reflectance UV/vis spectra of both as-prepared and thermally treated pure and silver-loaded hydrogen titanates. The band edge energies [3,185,298], due to the transition from the $\mathrm{O}^{-}$antibonding orbital to the lowest energy orbital of $\mathrm{Ti}^{4+}$, have been evaluated by plotting $(\alpha E)^{1 / 2}$ (where $\alpha$ and $E$ represent the absortion coefficient and energy, respectively) against the incident photon energy $(h v)$.

In particular, the spectrum of the $\mathrm{H}$-form titanate sample exhibits an absorption edge at $\sim 344 \mathrm{~nm}$, which is slightly downward shifted to $\sim 380 \mathrm{~nm}$ after thermal treatment at $773 \mathrm{~K}$. The same behavior can be observed for silver loaded samples. Similarly, the absorption edge of silver titanates, placed at $\sim 375 \mathrm{~nm}$, shifts to $385 \mathrm{~nm}$ after thermal treatment, thus confirming significant structural changes [233].

With regard to the metal loading effect on the absortion spectra, a red shift for silver loaded titanates nanotubes with respect to hydrogen titanates was observed. This could be related to the ion exchange process, promoted by $\mathrm{Ag}^{+}$, which favors the partial swelling of the outermost layers to occur. These data are in agreement with some reports that show that the frequency shift of quasi-2D crystallites is governed predominantly by their layer thickness [233]. Similar red shift was detected when Co was deposited at low coverage on $\mathrm{H}$ form titanate nanotubes (discussed above) [280]. Gold deposition also causes a significant decrease in band gap on H-form titanate nanowires and nanotubes, respectively due to partial ion exchange (see below and in Ref. [127]).

The diffuse reflectance UV/vis spectra of silver loaded titanates before and after solar irradiation are shown in Fig. 35. The absorption edge of silver loaded titanates at $375 \mathrm{~nm}$ (black curve) is slightly shifted downwards to $\sim 425 \mathrm{~nm}$ (dark gray curve) and $\sim 450 \mathrm{~nm}$ (light gray curve) after 250 and $900 \mathrm{~W}$ irradiation, respectively, while a broad and weak absorption band is simultaneously observed in the $450-550 \mathrm{~nm}$ range. Similar findings were published independently [299] about the photochromic behavior of silver titanates exposed to monochromatic radiation at different wavelengths. A relationship between the photochromic behavior and the formation of metallic silver particles of different sizes was found.

The FTIR spectra of $\mathrm{CO}$ adsorbed at $77 \mathrm{~K}$ at decreasing coverage, on the surface of titanate phases and on $\mathrm{TiO}_{2}$ surfaces, both pure and silver loaded, are presented in Fig. 36(a)-(d). The titanate sample outgassed at $450 \mathrm{~K}$ in vacuum is entirely hydroxylated, as shown in the inset of Fig. 36a, where intense 


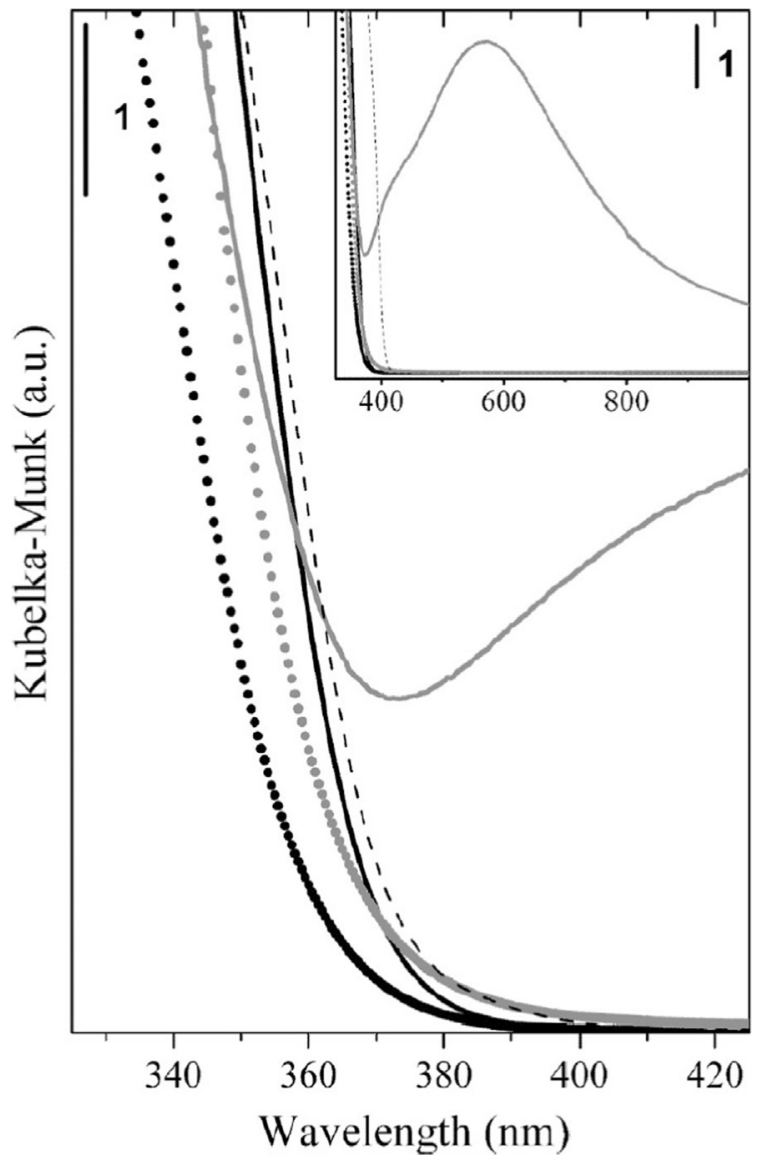

Fig. 34. UV/vis spectra of hydrogen titanate before (black dotted curve), and after thermal treatment at $773 \mathrm{~K}$ (black solid lines), Ag-titanate nanotubes before (gray dotted curve) and after thermal treatment at $773 \mathrm{~K}$ (gray solid curve), and anatase (-). Inset: surface plasmon resonance absortion of $\mathrm{Ag}$ titanates after thermal treatment at $77 \mathrm{~K}$. Reproduced from Ref. [233].

and broad bands in the $\mathrm{OH}$ region are observed. From this it can be derived that the peak at $2156 \mathrm{~cm}^{-1}$, slightly shifting downward with the coverage to $2152 \mathrm{~cm}^{-1}$, is due to $-\mathrm{OH}$...CO complexes on (020) and/or (110) crystalline planes of multiwalled titanate nanotubes, in agreement with the literature data [300]. The presence of a shoulder at $2178 \mathrm{~cm}^{-1}$ is due to $\mathrm{Na}^{+} \ldots \mathrm{CO}$ complexes formed on residual $\mathrm{Na}^{+}$ions [301]. With regard to the weak shoulder at $2130-2135 \mathrm{~cm}^{-1}$, it vanishes after short evacuation, which confirms its assignment to physically adsorbed CO [302].

The spectra of $\mathrm{CO}$ adsorbed on the sample calcined and outgassed at $773 \mathrm{~K}$ are reported in Fig. 36(b). The dominant peak at $2160 \mathrm{~cm}^{-1}$ is assigned to the mode of partially arranged CO oscillators adsorbed on fivefold coordinated $\mathrm{Ti}^{4+}$ sites on the main exposed $\mathrm{TiO}_{2}$ surfaces. The peak gradually shifts from 2160 to $2169 \mathrm{~cm}^{-1}$ as the CO coverage is decreased. This effect can be explained as a progressive vanishing of the dynamic and static lateral interactions among the $\mathrm{CO}$ oscillators. The final frequency $\left(2169 \mathrm{~cm}^{-1}\right)$ is associated with isolated $\mathrm{Ti}^{4+} \ldots \mathrm{CO}$ species.

More complex features are observed on silver loaded systems (Fig. 36(c)). In particular, besides the intense band at $2156 \mathrm{~cm}^{-1}$ due to the presence of hydroxyls, an intense peak at $2166 \mathrm{~cm}^{-1}$, shifting to $2171 \mathrm{~cm}^{-1}$, and a strong shoulder at

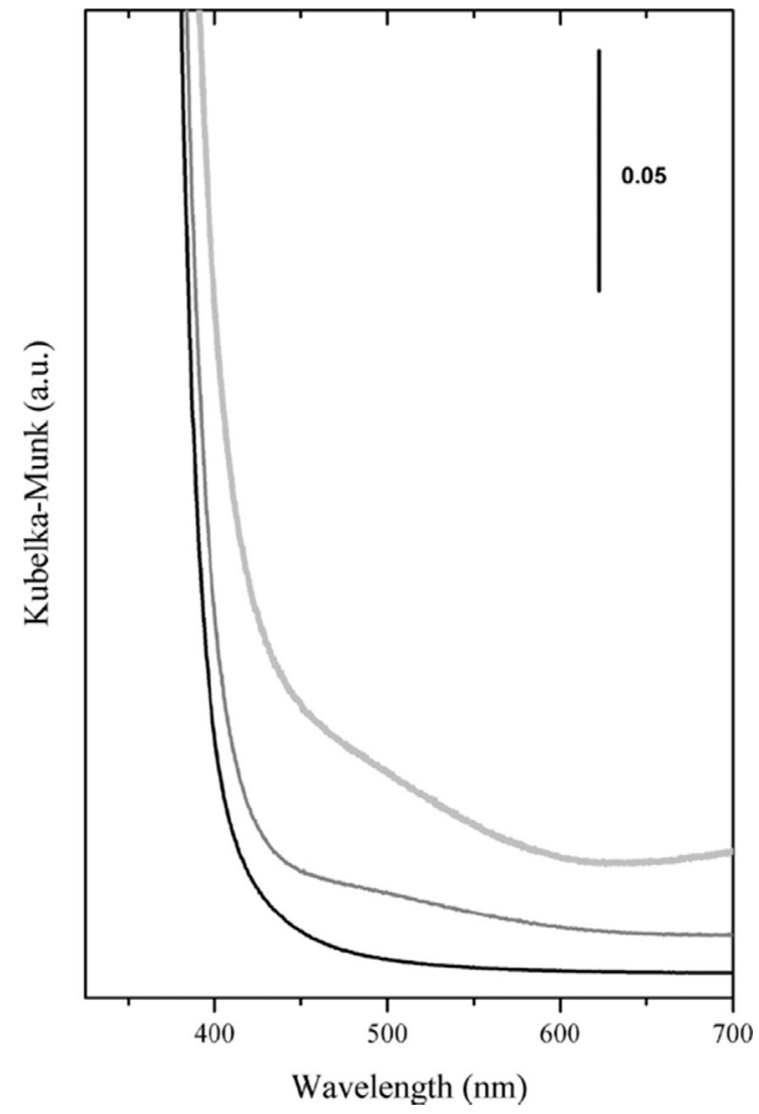

Fig. 35. UV/vis spectra of silver titanate before (black curve) and after solar light irradiation under $250 \mathrm{~W}$ (dark gray curve) and $900 \mathrm{~W}$ (light gray curve), respectively. Reproduced from Ref. [233].

higher frequency $\left(2184 \mathrm{~cm}^{-1}\right)$ can also be distinguished. The features in the $2166-2190 \mathrm{~cm}^{-1}$ region can be assigned to a variety of $\mathrm{Ag}^{\mathrm{\delta}}+\ldots \mathrm{CO}$ adducts uniformly distributed inside and on the external layers surrounding the nanotube cavity [233]. Notice that the $2184 \mathrm{~cm}^{-1}$ high frequency peak has been also observed on $\mathrm{Ag}^{+} \mathrm{ZSM} 5$ in the same frequency range and it was assigned to $\mathrm{Ag}^{+}(\mathrm{CO})$ and $\mathrm{Ag}^{+}(\mathrm{CO})_{2}$ adducts [303]. On silver exchanged titanate nanotubes, the formation of $\mathrm{Ag}^{+}(\mathrm{CO})_{n}$ $(n=1,2)$ species was revealed.

On silver doped samples outgassed at $773 \mathrm{~K}$ (Fig. 36(d)) the feature at $2166-2190 \mathrm{~cm}^{-1}$ (assigned to $\mathrm{Ag}^{+}$(CO) species) is eroded. This result is in agreement with the HRTEM results that show that the thermal treatment at $773 \mathrm{~K}$ favors the formation of silver particles [233]. A further component at $2179-2180 \mathrm{~cm}^{-1}$ is also observed at high coverages, which can be assigned to $\mathrm{Ag}^{\delta+} \ldots \mathrm{CO}$ adducts formed upon $\mathrm{CO}$ interaction with residual $\mathrm{Ag}^{+}$species', which have not be reduced to $\mathrm{Ag}^{0}$ during the thermal treatment.

\subsubsection{Characteriztion of $R h$ nanoparticles on titanate nano-} wires and nanotubes. Supported $\mathrm{Rh}$ is an important catalyst because $\mathrm{Rh}$ is one of the most effective metals in several technologically important reactions (mainly in the hydrogenation of $\mathrm{CO}_{2}$ ) and it was demonstrated that the nature of the support has a marked influence on its specific activity 


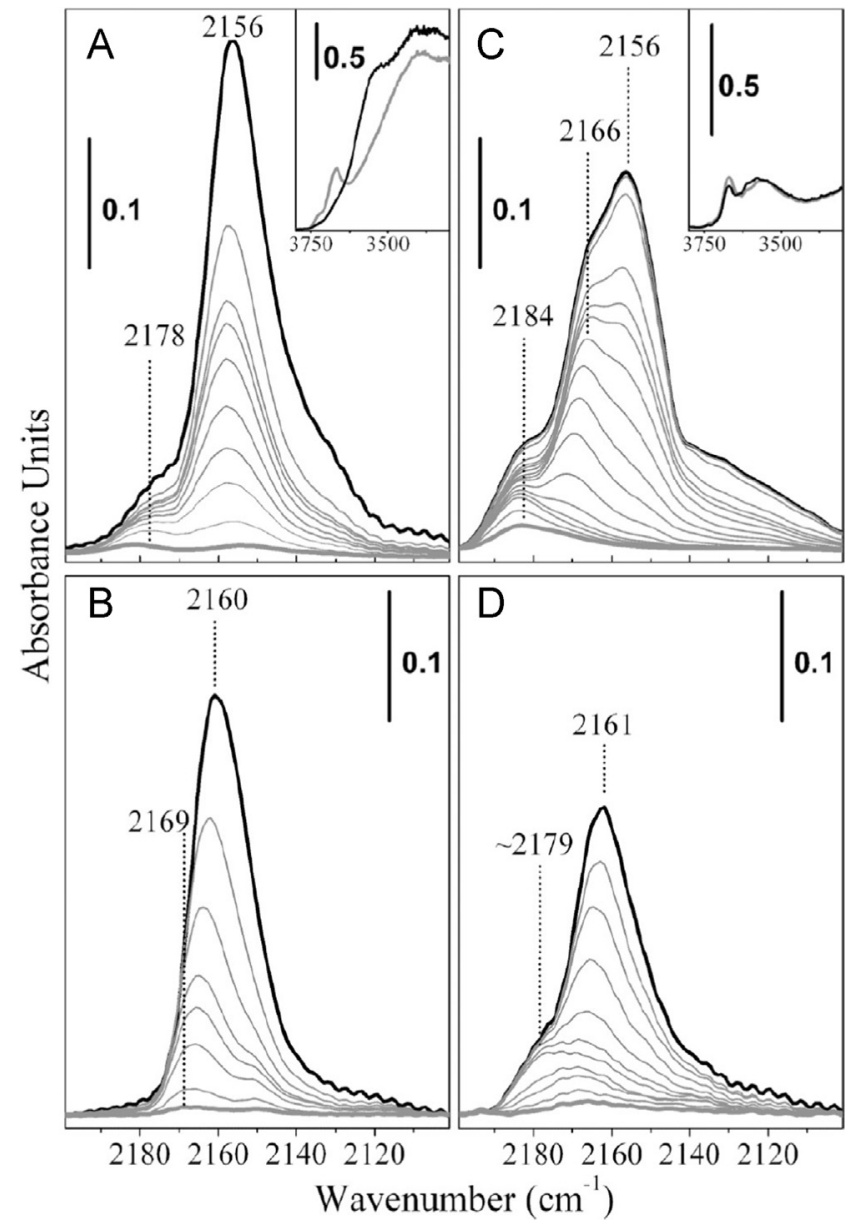

Fig. 36. FTIR spectra of $\mathrm{CO}$ adsorbed at $100 \mathrm{~K}$ at increasing coverages on $\mathrm{H}$-form titanates (a) before and (b) after thermal treatment at $773 \mathrm{~K}$; (c) Ag-titanates before and (d) after thermal treatment at $773 \mathrm{~K}$. Reproduced from Ref. [233].

[196,197,304,305]. Rh/titanate nanocomposites with different $\mathrm{Rh}$ concentrations were produced by impregnating titanate nanowires and nanotubes with mixtures of calculated volumes of $\mathrm{RhCl}_{3} \times 3 \mathrm{H}_{2} \mathrm{O}$ (Johnson Matthey) solutions to yield 1 and $2.5 \mathrm{wt} \%$ metal content. The impregnated powders were dried in air at $383 \mathrm{~K}$ for $3 \mathrm{~h}$. The final pre-treatment was at $473-$ $573 \mathrm{~K}$ in hydrogen atmosphere [75,181-183].

TEM images of Rh decorated nanowires (Fig. 37(A)) and nanotubes (Fig. 37(B)) thermally treated at $673 \mathrm{~K}$ show the presence of homogeneously dispersed nanoparticles on the surface of titanate nanostructures. The average diameter of nanoparticles is $4.9 \pm 1.4 \mathrm{~nm}$ and $2.8 \pm 0.7 \mathrm{~nm}$ in the case of nanowires and nanotubes, respectively, as evidenced by the corresponding size distributions. The difference in average particle diameter and distribution broadening could be due to differences in the crystal transformation process as discussed above. Moreover, surface diffusion and coalescence kinetics of $\mathrm{Rh}$ nanoparticles on the tubular and wire-like forms of titanate nanostructure can also be different. No reflections in the XRD patterns of Rh decorated nanostructures can be assigned to any $\mathrm{Rh}$ forms due to the relatively low concentration and small size of Rh particles. Nevertheless, the TEM electron diffraction study of samples heat-treated at $673 \mathrm{~K}$ (not shown here) confirmed the presence of metallic $\mathrm{Rh}$ in the sample because the characteristic diffraction rings at [hkl] $=[111],[200]$, [220], and [311], corresponding to the d-spacing values of $2.153 \AA$, $1.885 \AA, 1.327 \AA$ and $1.131 \AA$ in the $\operatorname{Rh}(0)$ fcc structure, respectively, could be identified [183].

It is important to mention that the original narrow size distribution is maintained even at relatively high temperatures (reduction temperature of the catalysts: $473-573 \mathrm{~K}$ ) on both nanowires and nanotubes. Rh clusters of controlled size can be prepared by physical vapor deposition (PVD) [192,306,307] and using $\mathrm{Rh}$ organometallic precursors [274,308] on $\mathrm{TiO}_{2}(110)$ as well. However, STM [306,309], XPS and LEIS [192] experiments revealed that depending on the original cluster size and the evaporation temperature, the agglomeration of $\mathrm{Rh}$ nanoparticles can be significant even below $500 \mathrm{~K}$ on that surface. The relatively small cluster sizes obtained on titanate nanowires and nanotubes may indicate that the metal diffusion on these nanobjects is limited compared to that on well-ordered titania.

Fig. 38 shows the binding energies of $\mathrm{Rh} 3 \mathrm{~d}$ orbitals in the case nanowires and nanotubes. Photoemission from the Rh $3 \mathrm{~d}$ peak centered at $309.3 \mathrm{eV}$ at $1 \% \mathrm{Rh}$ content and $308.3 \mathrm{eV}$ at $2 \%$ metal content clearly suggest the existence of an oxidation state or morphology different from bulk, as the metallic $\mathrm{Rh}$ photoemission for $\mathrm{Rh} 3 \mathrm{~d}_{5 / 2}$ is at $307.1 \mathrm{eV}$. Considering the nearly $2.2 \mathrm{eV}$ shift relative to metallic $\mathrm{Rh}$ in the case of $1 \% \mathrm{Rh}$ content (upper spectra of Fig. 38), this relative shift is $1.2 \mathrm{eV}$ for $2 \%$ metal content (bottom spectra of Fig. 38) although we cannot exclude a certain peak broadening effect due to the width of the nanoparticle diameter distribution. The higher binding energy states may correspond to very small clusters stabilized in the structure of nanowires and nanotubes. The binding energy is influenced by the relaxation energy and this "final-state" effect depends on the particle size [292]. Smaller metal loading presumably leads to the formation of smaller particles (higher binding energy). This could explain the observed differences in the intensity ratios of the higher binding energy peaks of samples decorated with smaller (1\% metal loading) and larger (2\% metal loading) $\mathrm{Rh}$ nanoparticles. The intensities and positions of higher binding energy peaks may depend on the rate of electron transfer from metal to substrate (formation of partially positive charged $\mathrm{Rh}$ ), too.

The stabilization of $\mathrm{Rh}$ clusters in small size and the influence of $\mathrm{Rh}$ nanoparticles on the transformation of titanate structures can be explained also by the electronic interaction between $\mathrm{Rh}$ and titanate, which was observed in several cases between reduced titania and metal including Rh [310-313]. Due to the preparation methods of titanate nanostructures and the mild reduction of $\mathrm{Rh} /$ titanates, the nanowires and nanotubes may contain significantly more defects than commercially used reduced titania. The presence of a high number of defects and oxygen vacancies in titanate could initiate an increased electron flow between metal and titanates.

Recently titanium dioxide $\left(\mathrm{TiO}_{2}\right)$ (110) surfaces with $\mathrm{Pt}$ adatoms were examined using a noncontact atomic force microscope (NC-AFM) and Kelvin probe force microscope 
A
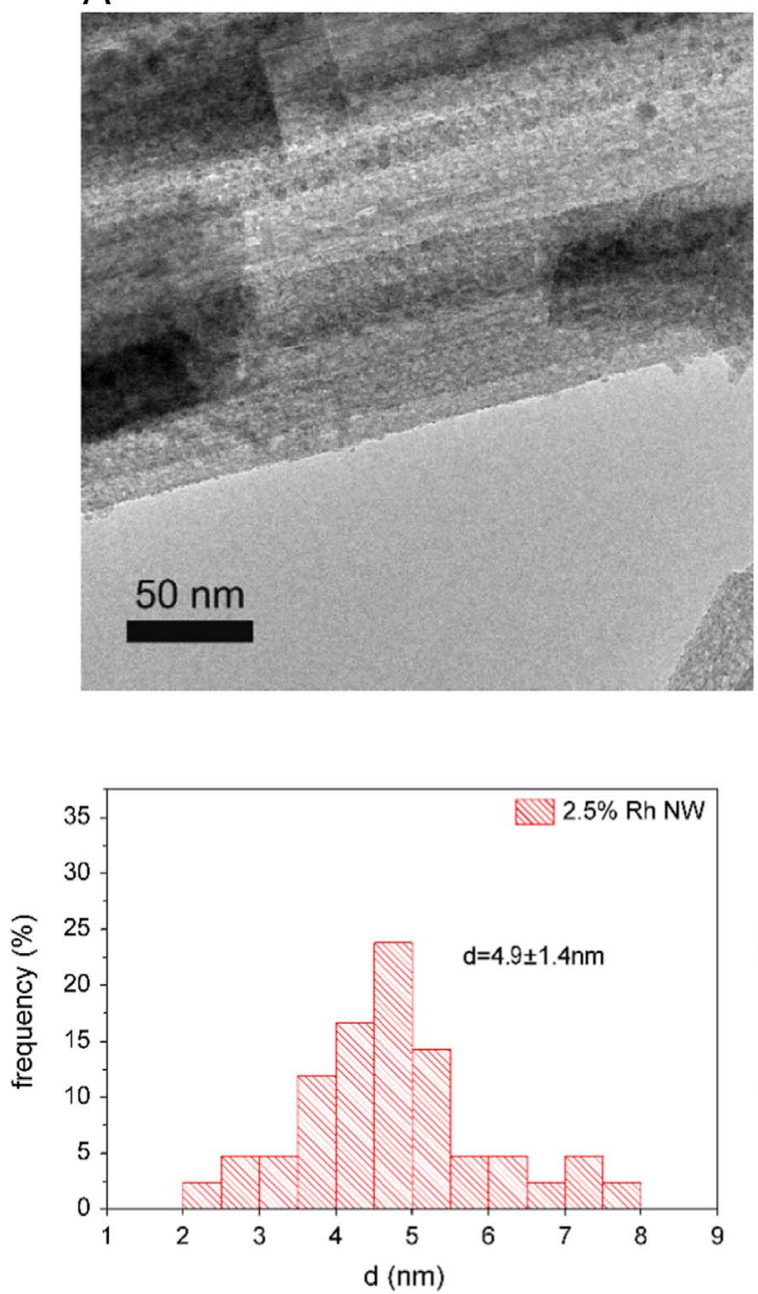

B
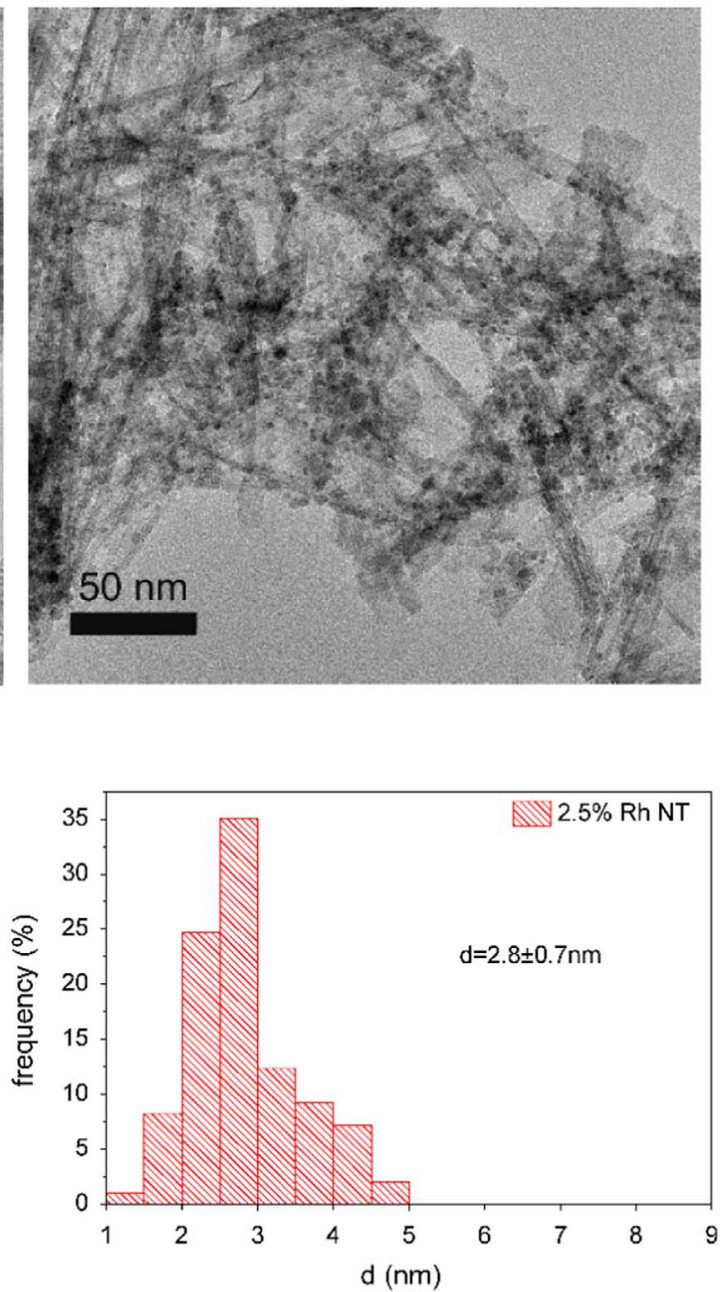

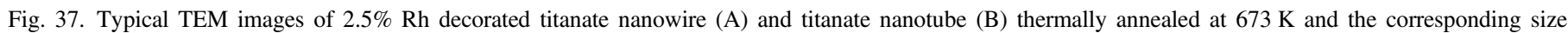
distribution of Rh nanoparticles. Reproduced from Ref. [183].

(KPFM). Platinum clusters with diameters of 2-3 nm were obtained. The work function decrease was interpreted with an electric dipole moment directed towards the vacuum, as a result of electron transfer from the adatom to the $\mathrm{TiO}_{2}$ substrate [314,315]. Similar phenomena may occur in the case of Rh-titanate nanosystems. Rh adatoms on Ti atoms and $\mathrm{O}$ atoms are mobile, while the adatoms in the $\mathrm{O}$ atom vacancies are not. A rhodium adatom in an $\mathrm{O}$ atom vacancy is in contact with two $\mathrm{Ti}$ atoms and, therefore, the electron transfer can be enhanced. This electron transfer could manifest in the observed higher binding energy state of metal in XPS, too.

On the other hand, ion exchange between protonated titanates and rhodium cannot be excluded similarly to silver on titanate nanotubes [233], Co on nanowires [280] and $\mathrm{Au}$ on both nanowires and nanotubes [52]. The ion exchange process could be supported by that fact that the band gap decreased by $0.2 \mathrm{eV}$ during the $\mathrm{Rh}$ doping on titanate nanotubes. The morphology of $\mathrm{Rh}$ supported on titanate nanowires and nanotubes was investigated by FTIR, employing adsorbed $\mathrm{CO}$ as a probe molecule sensitive to the local surface structure $[181,182]$. On rhodium-decorated nanowires the twin form was dominant (as evidenced by the IR peaks at 2028 and $2097 \mathrm{~cm}^{-1}$ ) indicating the presence of single atoms or very small clusters. It was assumed that this type of rhodium represents metal (with positive charge) in ion exchange positions. This ion exchange process also leads to the appearance of higher binding energy states in XPS. It could be supposed that there are differences in the nature of the Rh-nanowire and Rh-nanotube metal-support interfaces due to slight differences in cluster size, in the rate of electron transfer from metal to support or in the rate of ion exchange.

The $\mathrm{CO}$ adsorption at $300 \mathrm{~K}$ caused a significant change in $\mathrm{Rh} 3 \mathrm{~d}$ core level spectra on $\mathrm{Rh}$ loaded titanate sample [181]. After CO adsorption the intensity of high energy tailing increased significantly (Fig. 39). The figure shows the spectrum before and after 60 min $\mathrm{CO}$ adsorption on Rh deposited on titanates nanowires. The peak centered at $309.1 \mathrm{eV}$ clearly shows the formation of smaller clusters, therefore, the $\mathrm{CO}$ induced the disruption of rhodium to smaller sizes on nanowires in the activated process.

Earlier, disruption on oxide-supported rhodium was observed by infrared spectroscopy [316]. The adsorption of 

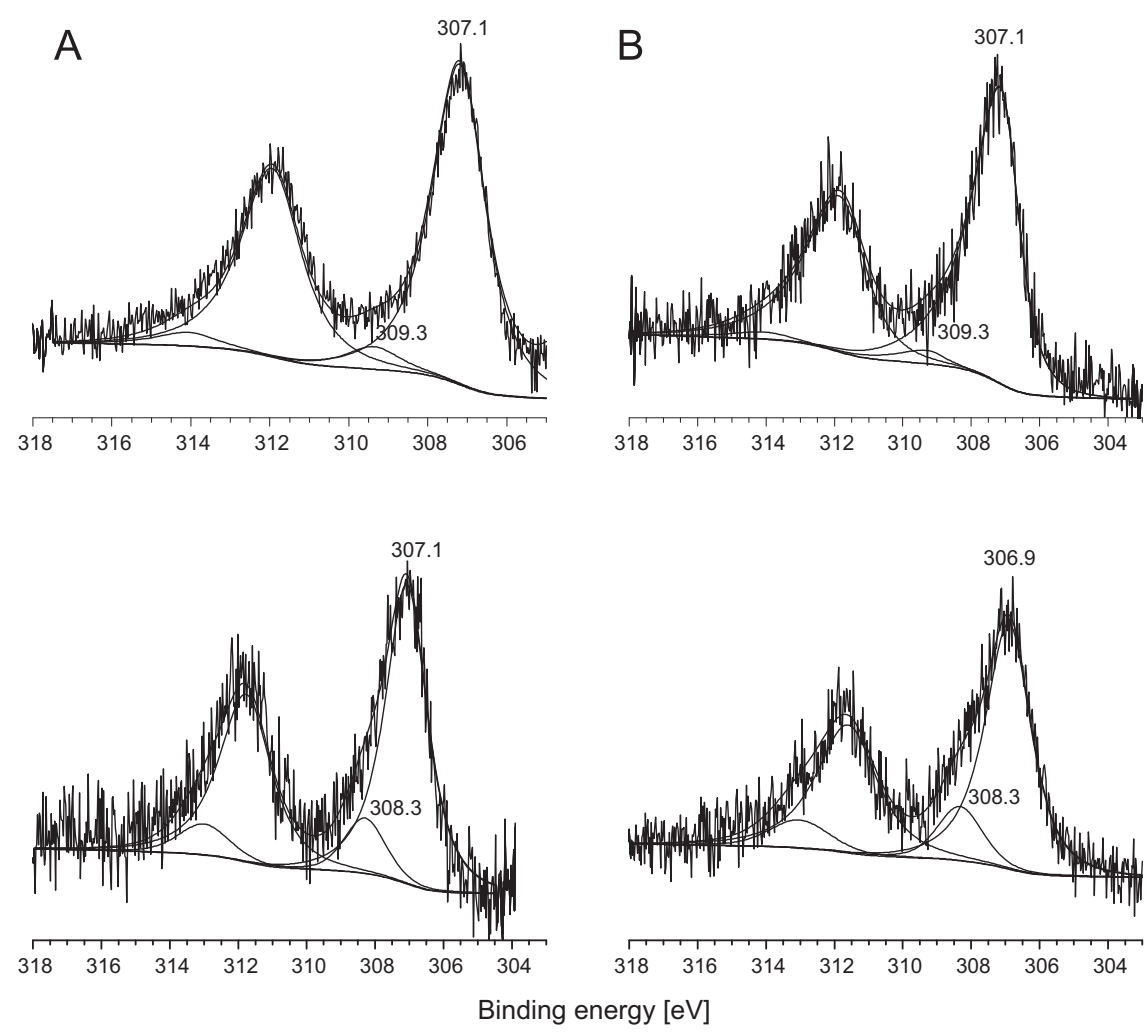

Fig. 38. XP spectra of Rh 3d on titanate nanowire (A) and nanotube (B) with $1 \%$ Rh content (upper spectra) and $2 \%$ metal content (lower spectra). Reproduced from Ref. [183].

$\mathrm{CO}$ on alumina-supported $\mathrm{Rh}$ of different crystallite sizes produced by reduction at $573-1273 \mathrm{~K}$ has been investigated by monitoring the development of the infrared bands assigned to $\mathrm{Rh}^{+}(\mathrm{CO})_{2}$ and $\mathrm{Rh}-\mathrm{CO}$ species. It was concluded that the effect of $\mathrm{CO}$ on the state of dispersed $\mathrm{Rh}$ is twofold. In harmony with the EXAFS measurements of Prins et al. [317], $\mathrm{CO}$ adsorption at around $300 \mathrm{~K}$ leads to the disruption of $\mathrm{Rh}$ clusters and the formation of isolated $\mathrm{Rh}^{+}$sites, as indicated by the slow development of gem-dicarbolyl. Above $423 \mathrm{~K}$ another effect of adsorbed $\mathrm{CO}$ comes into prominence, which leads to the formation of $\mathrm{Rh}$ crystallites at the expense of isolated $\mathrm{Rh}^{+}$sites. Recently the disruption of $\mathrm{Rh}$ to smaller size particles was confirmed by STM on $\mathrm{Rh} / \mathrm{TiO}_{2}(110-$ $(1 \times 2))$ surface [318].

The morphology of Rh supported on titanate nanowires and nanotubes was investigated by FTIR, employing adsorbed CO as a probe molecule sensitive to the local surface structure [181]. Adsorbed CO exhibits at least three different stretching frequencies belonging to certain adsorption sites of $\mathrm{Rh}$ oxide support $[316,319,320]$. The band at $2070-2030 \mathrm{~cm}^{-1}$ is due to CO being linearly coordinated to $\mathrm{Rh}^{0}$, the band at $\sim 1885$ $\mathrm{cm}^{-1}$ represents the bridge bonded $\mathrm{CO}\left(\mathrm{Rh}_{2}-\mathrm{CO}\right)$ and the features at $\sim 2100$ and $\sim 2020 \mathrm{~cm}^{-1}$ correspond to the symmetric and asymmetric stretching of $\mathrm{Rh}^{+}(\mathrm{CO})_{2}$ (twin $\mathrm{CO}$ ), respectively. These latter IR signals were detected when the crystallite size was very small [316]. On titanate nanowires the twin form was dominant while on nanotubes the linearly adsorbed CO also showed up [181]. From these IR studies it can be concluded that a significant part of Rh exists in small particle sizes on both nanowires and nanotubes.

\subsubsection{Formation and characterization of gold nanoparticles} and nanoclusters on titanate nanostructures. $\quad \mathrm{Both}^{\mathrm{TiO}} \mathrm{O}_{2}$ and titanate nanostructures modified by gold nanoparticles are promising materials from the heterogeneous low-temperature and photocatalytic points of view. Morphology and chemical nature of gold additives on titanate nanotubes and nanowire are the keys to understand the processes driven by gold containing nanomaterials.

Although it is virtually impossible to prepare a truly monodisperse collection of metallic particles (i.e. particles of exactly the same diameter), in the past years significant progress was made in the preparation of gold particles with a narrow diameter distribution between $\sim 2$ and $10 \mathrm{~nm}$ [321324]. Titanate nanostructures were decorated by gold nanoparticles in this paragraph by two methods: (A) the Aucontaining $\mathrm{H}$-form titanate nanowires and nanotubes with the same actual Au loading $\left(\mathrm{HAuCl}_{4}, 1-2.5 \%\right)$ were obtained by deposition-precipitation method $[181,321,322]$ at $\mathrm{pH}=7$ and at $343 \mathrm{~K}$, followed by treatment in $\mathrm{H}_{2}$ at $473 \mathrm{~K}$. When the reduction temperature was $573 \mathrm{~K}$, we did not observe any changes in particle sizes and morphology. The second method (B) corresponded to reducing gold with $\mathrm{NaBH}_{4}$. The sodium borohydride reduction of metal ions is a known chemical route to synthesize metal nanoparticles $[325,326]$. $\mathrm{HAuCl}_{4}$ solution with an appropriate concentration to provide $2.5 \mathrm{wt} \%$ gold 

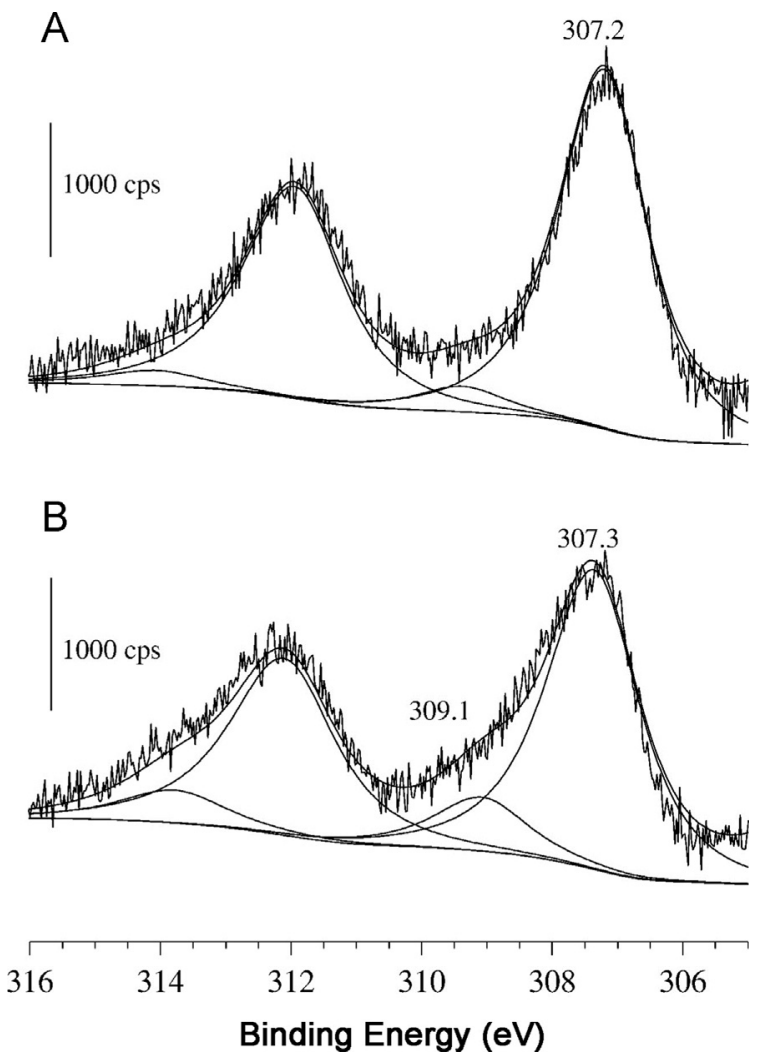

Fig. 39. Rh 3d XP spectra before (a) and after $60 \mathrm{~min}$ CO adsorption, $P=1$ bar (b). Reproduced from Ref. [181].

loading was added to the well-homogenized nanowire or nanotube suspension. After $10 \mathrm{~min}$ of stirring $50 \mathrm{mg}$ of $\mathrm{NaBH}_{4}$ (separately dissolved in $5 \mathrm{ml}$ of distilled water) was added rapidly to achieve the instantaneous formation of gold nanoparticles at $293 \mathrm{~K}$. The suspension was stirred further for $20 \mathrm{~min}$ then rinsed with distilled water thoroughly. The aspurified sample was dried overnight in air in a temperature programmed electric oven at $343 \mathrm{~K}$. Quantitative energydispersive X-ray spectroscopic (EDX) and XPS analysis showed that after washing, the bulk and surface gold concentrations decreased by about 55\%. Trace amounts of $\mathrm{Na}$ remained in the sample after these post-synthetic treatments. Boron was undetectable in the samples [52].

Fig. 40 shows typical HRTEM images obtained with two different reduction procedures on $\mathrm{H}$-form nanowires. In the case of hydrogen reduction (Fig. 40(A)), the size of $\mathrm{Au}$ nanoparticles was mostly between 3 and $10 \mathrm{~nm}$, but a small fraction was observed at around $15-20 \mathrm{~nm}$ as well. A very similar result was obtained on $\mathrm{H}$-form titanate nanotubes. HRTEM and XRD measurements indicated that wellcrystallized face-centered cubic gold particles were dispersed on both nanowires and nanotubes. The concentration of the gold precursor slightly affected the gold diameter distribution in the 1-2.5 wt $\%$ gold content range. The average size of gold was significantly smaller when the reduction was executed by $\mathrm{NaBH}_{4}$ (Fig. 40(B)). In this way we obtained a homogeneous distribution: the size of Au nanoparticles was between 3.0 and $8.0 \mathrm{~nm}$ on the $\mathrm{H}$-form titanate nanowires. The gold size distribution was very similar on the nanotubes as well, however, a small fraction of the nanoparticles had a diameter of approx. $10 \mathrm{~nm}$.

The chemical environment of the prepared gold nanoparticles was characterized by XPS. The $4 \mathrm{f}$ spectra of gold deposited on titanate nanostructures after different reduction procedures and at different gold loading are displayed in Fig. 27. For comparison the figure shows the clean gold film (thickness: $50 \mathrm{~nm}$ ) prepared on a glass plate. In the $4 \mathrm{f}$ region symmetric $4 \mathrm{f}_{5 / 2}$ and $4 \mathrm{f}_{7 / 2}$ emissions were detected (Fig. 41(A)). However, the features obtained on titanate nanostructures are quite different (Fig. 41(B)-(D)) insofar as two peaks are present in the reduced sample spectra for $\mathrm{Au} 4 \mathrm{f}_{7 / 2}$ at $84.0 \mathrm{eV}$ (metallic state) and at $85.8-86.3 \mathrm{eV}$ [52].

Two different explanations can be offered for the appearance of this unusually high binding energy gold state. Core level shifts due to particle size must be considered first in the interpretation of the spectra of nanoparticles [292,327-329].

Although this effect undoubtly plays a role in the present case, the observed nearly $2 \mathrm{eV}$ binding energy shift cannot be explained satisfactorily on this ground alone. The systematic increase of the binding energy with decreasing cluster size was obvious on $\mathrm{TiO}_{2}(110)$ [193]. The $\mathrm{Au} 4 \mathrm{f}_{7 / 2}$ peak positions were measured as a function of Au coverage. The peak appeared at $84.3 \mathrm{eV}$ at very low coverage ( $0.04 \mathrm{ML})$ on the stoichiometric $\mathrm{TiO}_{2}(110)$ surface. The position of this emission shifted slightly to lower binding energy with increasing coverage. Above $1 \mathrm{ML}$ coverage it was located at $84.0 \mathrm{eV}$, which corresponds to the bulk position. The observed shift can be attributed to the finite size of the clusters combined with the insulating nature of the substrate, which results in a less efficient screening of the core hole formed in the photoemission process. On reduced $\mathrm{TiO}_{2}(110)$ the binding energy shift from submonolayer to monolayer is larger $(>0.6 \mathrm{eV})$ than that on the stoichiometric surface as indicated by the $\mathrm{Au} 4 \mathrm{f}_{7 / 2}$ spectra recorded at different gold coverages. It was shown experimentally that increasing the number of surface defects could enhance the nucleation probability. The deposited Au forms smaller size crystallites in higher density on thermally reduced or $\mathrm{Ar}^{+}$ion sputtered surfaces relative to the stoichiometric substrate $[327,330]$. Although this effect certainly plays a role in the present case as well since titanate nanowires and nanotubes obviously contain a large number of defects and irregularities, the observed nearly $2 \mathrm{eV}$ binding energy shift cannot be explained satisfactorily on his ground alone.

The second possible explanation is that Au may have undergone an ion exchange process. This is not possible on $\mathrm{TiO}_{2}(110)$ because of the lack of cations compensating the framework charge, however, it is quite likely to happen on titanates which are well-known for their ion-exchange ability [246]. The cationic character of gold $\left(\mathrm{Au}^{+}\right)$represents a higher binding energy position in titanates compared to metallic gold state. When Au deposited as $\mathrm{Au}^{+}$ions at $1 \mathrm{eV}$ impact energy on rutile $\mathrm{TiO}_{2}$ samples was studied by XPS [331], a high binding energy state at $85.8 \mathrm{eV}$ was also observed, which further supports the explanation for the positively charged Au. Moreover, XPS showed partially charged $\mathrm{Au}^{+}$species with 

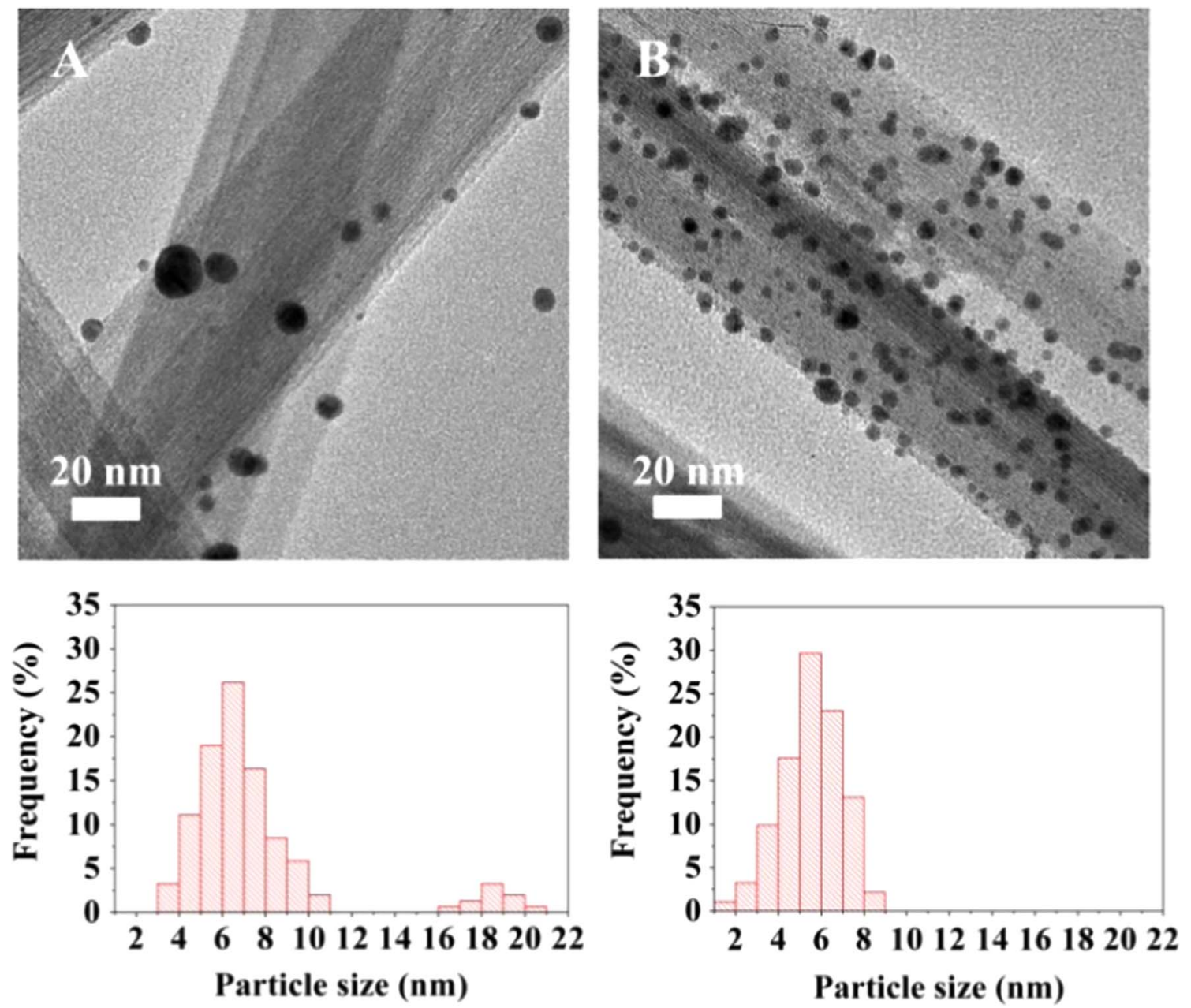

Fig. 40. HRTEM image on Au decorated H-form titanate nanowires obtained with $\mathrm{H}_{2}$ reduction (A) and obtained with $\mathrm{NaBH}_{4}$ reduction (B). The corresponding particle size distributions are also shown. The gold contents were $2.5 \mathrm{wt} \%$ in both cases. Reproduced from Ref. [52].

binding energy of $84.3-84.6 \mathrm{eV}$ by deposition on nanoceria at low coverages [332]. It was shown that nanoceria stabilizes small Au clusters, which may even be incorporated into ceria nanoparticles.

It can be seen in Fig. 41 that the relative intensity of the higher binding energy peak is larger at $1 \mathrm{wt} \%$ gold loading that at $2.5 \mathrm{wt} \%$ content (Fig. 41(B) and (C)). This means that at lower loading relatively more gold atoms can occupy ion exchange positions $(86.1-86.3 \mathrm{eV})$. It is remarkable that the milder reducing agent, $\mathrm{H}_{2}$, results in a higher portion of $\mathrm{Au}^{+}$/ $\mathrm{Au}^{0}$ at the same loading; relatively less gold occupies the ion exchange position after reduction with $\mathrm{NaBH}_{4}$ (Fig. 41(D)). It is very likely that the strong reducing agent reduces the positively charged gold from the ion exchange positions to metallic states during the reaction. Comparison of the spectra obtained at low and higher loading (Fig. 41(B) and (C)) might indicate that $\mathrm{Au}^{3+}$ is reduced to $\mathrm{Au}^{+}$and extensively participates in ion exchange with titanate at a competitive rate with the reduction to $\mathrm{Au}^{0}$ which results particle formation. Recently a thiol-protected $\mathrm{Au}_{25}$ crystal structure was characterized by X-ray crystallographic analysis and optical absoption measurements supported by theoretical calculations $[333,334]$ and tested in $\mathrm{CO}$ oxidation reaction [335]. Form the UV-vis spectrum (Fig. 42) it can be concluded (see below) that very small clusters, for example $\mathrm{Au}_{25}$ clusters, might also be formed and stabilized in the titanate framework, especially at lower Au loading, however, this sub-nm fraction cannot be detected by TEM applied.

An XPS-independent proof for the ion exchange can be obtained by measuring the band gap energy [52]. The band gap for pure titanate nanowires and nanotubes was 3.10 and $3.07 \mathrm{eV}$, respectively, while that for Au-doped titanate nanowire was less: $2.84 \mathrm{eV}$ at $1 \mathrm{wt} \%$ and $2.74 \mathrm{eV}$ at $2.5 \mathrm{wt} \% \mathrm{Au}$ content. Similar decreases in band gap energy were measured for gold containing nanotubes $(2.67 \mathrm{eV}$ at $2.50 \mathrm{wt} \% \mathrm{Au}$ content). When the gold was reduced by $\mathrm{NaBH}_{4}$ at $293 \mathrm{~K}$, these values were $2.74 \mathrm{eV}$ and $2.83 \mathrm{eV}$ for nanowires and nanotubes, respectively. In Fig. 42 some K-M function vs. $h \nu$ are displayed. The characteristic plasmonic band of gold could potentially overlap or merge with the $\mathrm{O} 2 \mathrm{p} \rightarrow \mathrm{Ti} 3 \mathrm{~d}$ CT band. The frequency and intensity of plasmonic bands change with particle size $(>5 \mathrm{~nm})$ and form. Gold nanocrystals typically show a collective electron excitation mode, surface plasmon resonance at $\sim 2.4 \mathrm{eV}$ for spherical particles. Small gold nanoparticles $(<3 \mathrm{~nm})$ lose their bulk-like electronic properties [336]. For more exact band gap determination, the 
$\mathrm{Au} 4 \mathrm{f}$
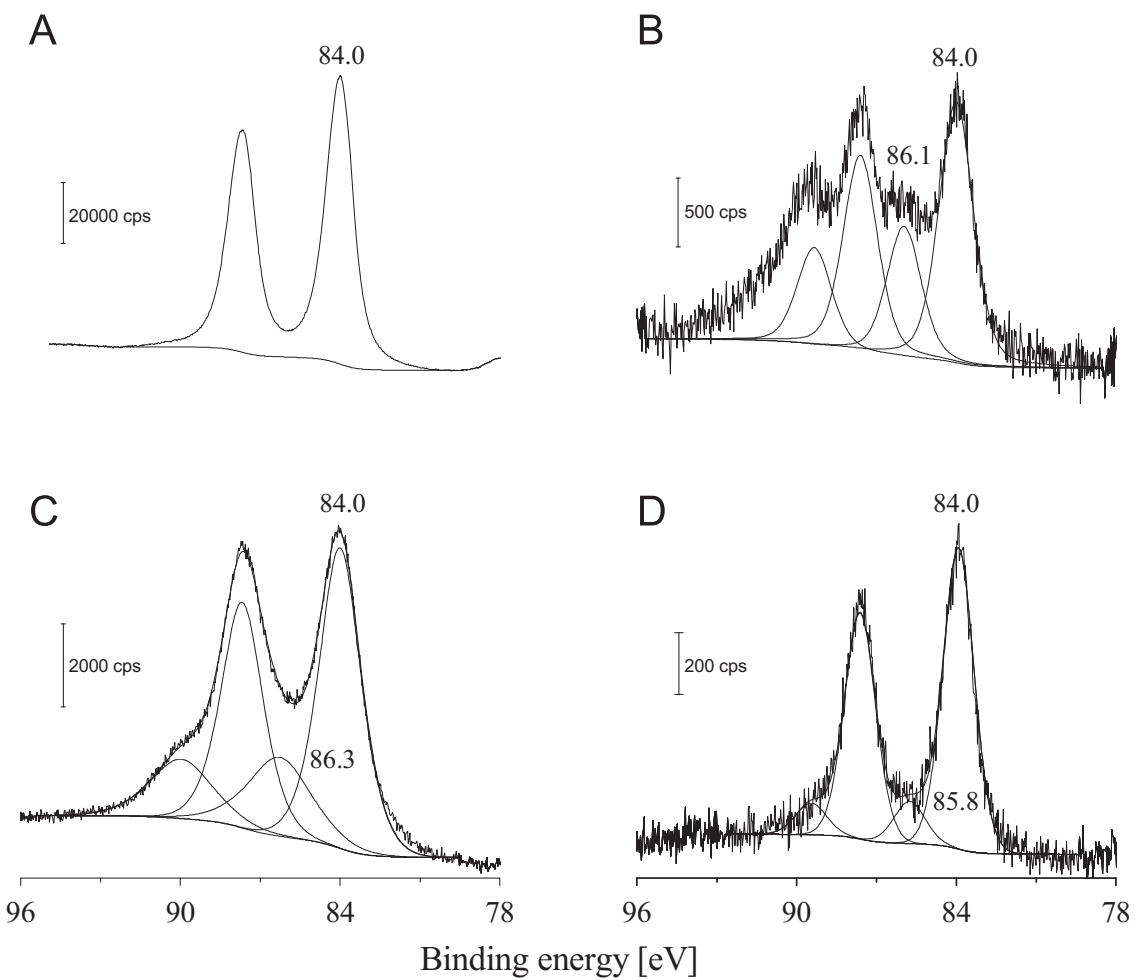

Fig. 41. Au 4f XPS on differentially prepared Au/titanate samples: (A) clean Au film; (B) 1 wt $\%$ Au/titanate nanowire obtained by $\mathrm{H}_{2}$ reduction at $473 \mathrm{~K}$; (C) $2.5 \mathrm{wt} \%$ Au/titanate nanowire obtained by $\mathrm{H}_{2}$ reduction at $473 \mathrm{~K}$; and (D) $2.5 \%$ Au/titanate nanowire obtained by $\mathrm{NaBH}_{4}$ reduction at $293 \mathrm{~K}$. $\mathrm{Reproduced}$ from Ref. [52].

absorption spectrum obtained at low Au loading is decomposed (Fig. 42(F)). The broad band between 2.5 and $3.2 \mathrm{eV}$ may be associated with the structure of the gold clusters. Recently it was published that the $\mathrm{Au}_{25}$ cluster exhibits multiple molecular-like transitions in its optical absorption spectrum: at least three well-defined bands at 1.8, 2.75 and $3.1 \mathrm{eV}$ were observed in the UV-vis spectrum [334].

The pronounced decrease of the band gap of titanate nanostructures and upon loading with Au suggests a very strong electronic interaction between the titanate nanostructure framework and gold, which may eventually result in an ion exchange process. It can be concluded that the higher binding energy XPS peak $(85.8-86.4 \mathrm{eV})$ is due primarily to gold located in cation positions and - to a lesser extent - to gold clusters which are in well-dispersed form (for example in $\mathrm{Au}_{25}$ structures) and it might be stabilized in the titanate framework. The ratio of these two components depends on the metal content and the preparation method. Remarkable conclusions can be drawn from the XPS intensity changes during heat treatment (Fig. 43). When the gold containing nanowires $(2.5 \%)$ were treated at $473 \mathrm{~K}$ and at $673 \mathrm{~K}$ in vacuum for $60 \mathrm{~min}$, the higher binding energy peak remained at $86.4 \mathrm{eV}$, however, its intensity decreased only slightly (Fig. 43(A)). This means that the heat treatment did not induce any significant gold segregation from ion exchange position. Interestingly, lower Au 4f XPS intensities were measured in the case of nanotubes at same metal loadings (Fig. 43(B)). It is very likely that a significant part of gold clusters occupies appropriate positions in the inner channel of the tube instead of the outer nanotube shell. A rough semi-quantitative XPS analysis indicates that nearly $75 \%$ of the gold could be located inside the tube. Further intensity changes $(50 \%)$ were measured upon heat treatment. This change is most likely due to further gold penetration into the tube as well as to the morphological transformation of the tube structure induced by gold adatoms.

\subsubsection{Plazmonic characteriztion of supported nanoparticles} and main features of clusters. Semiconductor-metal composites were suggested for a wide variety of applications recently, for example, they are widely employed in photocatalysis. It was demonstrated above that titanate nanowires and nanotubes can be successfully modified by metals. These modifications may result in better catalytic performances and more appropriate photocatalysts for environmental applications. Although a metal in contact with a titania or titanate semiconductor greatly enhances the overall photocatalytic efficiency, the role it plays in the interfacial charge-transfer processes is yet to be understood fully.

Nanoparticles of $\mathrm{Au}$ and $\mathrm{Ag}$ have been often coupled to $\mathrm{TiO}_{2}$ to utilize their property of the localized surface plasmonic resonance (LSPR) in photocatalysis [337,338]. It is supposed that LSPR may exist on titanate phases, too. A recent theoretical study on noble metals $(\mathrm{Pt}, \mathrm{Pd}, \mathrm{Ru}$ and $\mathrm{Rh})$ a recent 

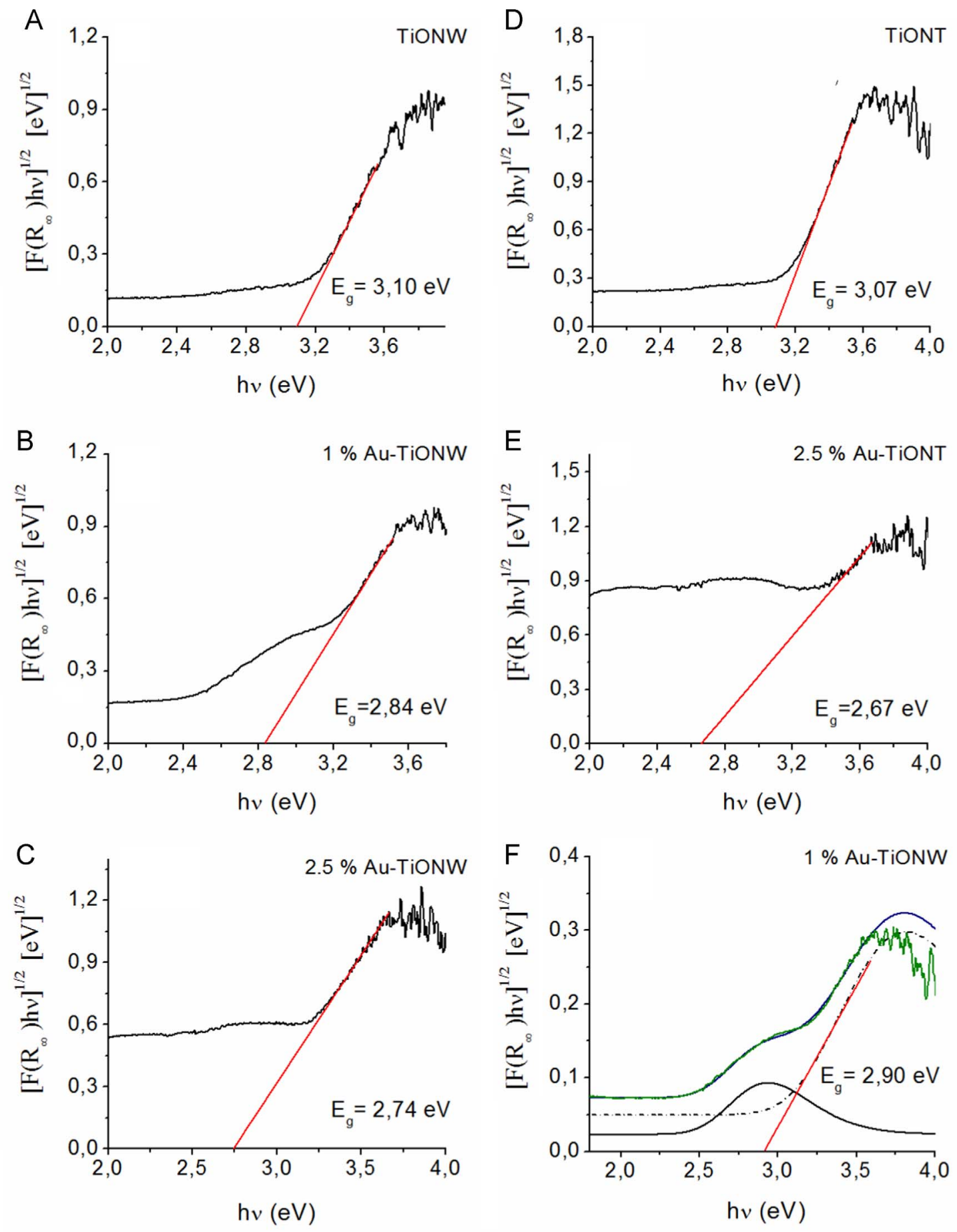

- Suggested absorbance of AuNC's (Gauss, $422 \mathrm{~nm}, 3.03 \mathrm{eV}$ ) - Au-TiONW -..... Suggested absorbance of Au-TiONW (Gauss) — Sum of Gauss fit

Fig. 42. Plots of Kubelka-Munk function vs. wavelength of titanate nanowires (A) and nanotubes (D) and Au-loaded titanate nanowires (Au-TiONW) (B), (C) and titanate nanotubes (Au-TiONT) (E). The decomposed spectrum of $1 \%$ Au-TiONW is also shown in (F). Reproduced from Ref. [52].

theoretical study has found that only Rh has a strong UV plasmonic response $[338,339]$. It is possible to fabricate $\mathrm{Rh}$ nanoclusters smaller than $10 \mathrm{~nm}$ through chemical means [340]. Polydisperse $\mathrm{Rh}$ nanoparticles have recently shown promise for UV plasmonics, but controlling the size and morphology of metal nanoparticles is essential for tuning surface plasmon resonances [341]. $\mathrm{Au}, \mathrm{Ag}$ and $\mathrm{Rh}$ supported on titania and titanate are now under intensive investigation as potential visible and UV sensitizers [341,342]. LSPR is the collective free electron charge oscillation in the metallic nanoparticles ( $>5 \mathrm{~nm}$ ), and strongly depends on particle size, shape and local dielectric environment [343,344]. Under LSPR excitation, a plasmon can excite the electrons in the conduction band to produce highly energetic "hot electrons". These "hot 
A

nanowire
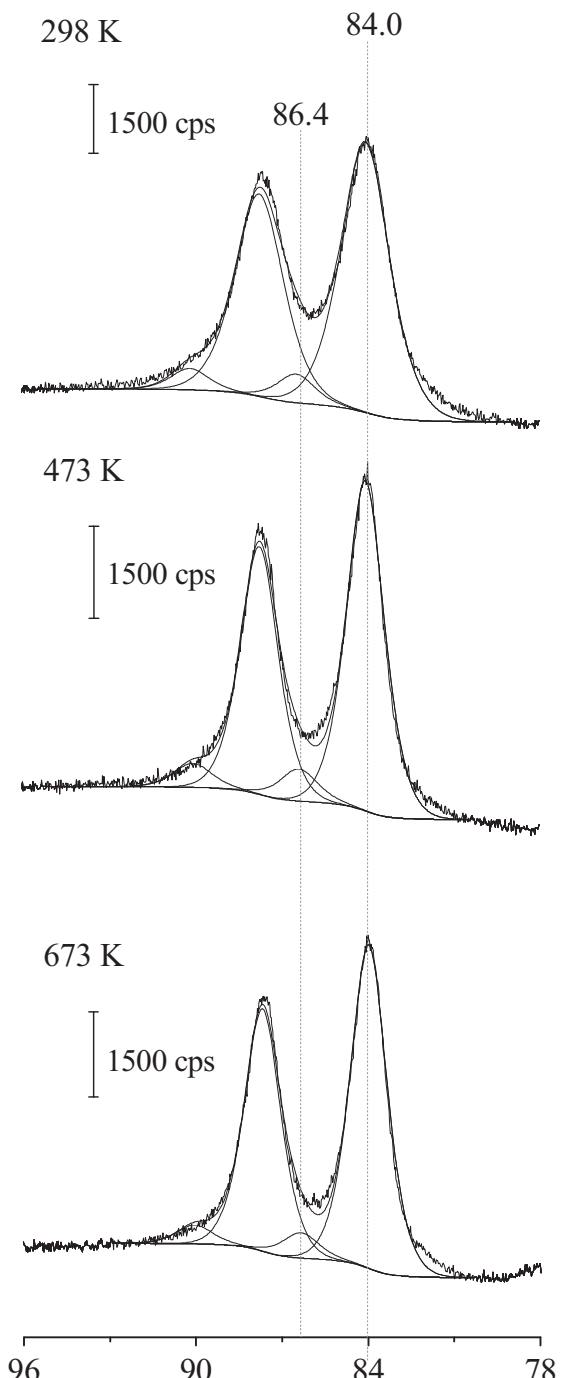

B

nanotube

Binding energy $[\mathrm{eV}]$

Fig. 43. Au 4f XPS on Au loaded H-form titanate nanowires (A) and nanotubes (B) at $2.5 \mathrm{wt} \%$ gold content after heat treatment in vacuum for $60 \mathrm{~min}$. Reproduced from Ref. [52].

electrons" can escape from the plasmonic nanostructures and transfer to a semiconductor (titania, titanates) in contact with the metal, thereby forming a metal-semiconductor Schottky junction $[335,345]$.

Smaller nanoparticles $(<3 \mathrm{~nm})$ lose their bulk-like electronic properties; for example, they no longer support the plasmon excitation characteristic of relatively large gold nanocrystals $(>3 \mathrm{~nm})$ as it was discussed and presented earlier. In addition, the atomic packing mode in small metal nanoparticles (also called clusters) plays an important role in their structure-property correlations and their size dependent properties [334]. The transition from the cluster state to the nanocrystalline states raises a number of fundamental questions, such as how the discrete electronic states develop into the collective plasmon state as the cluster size increases and

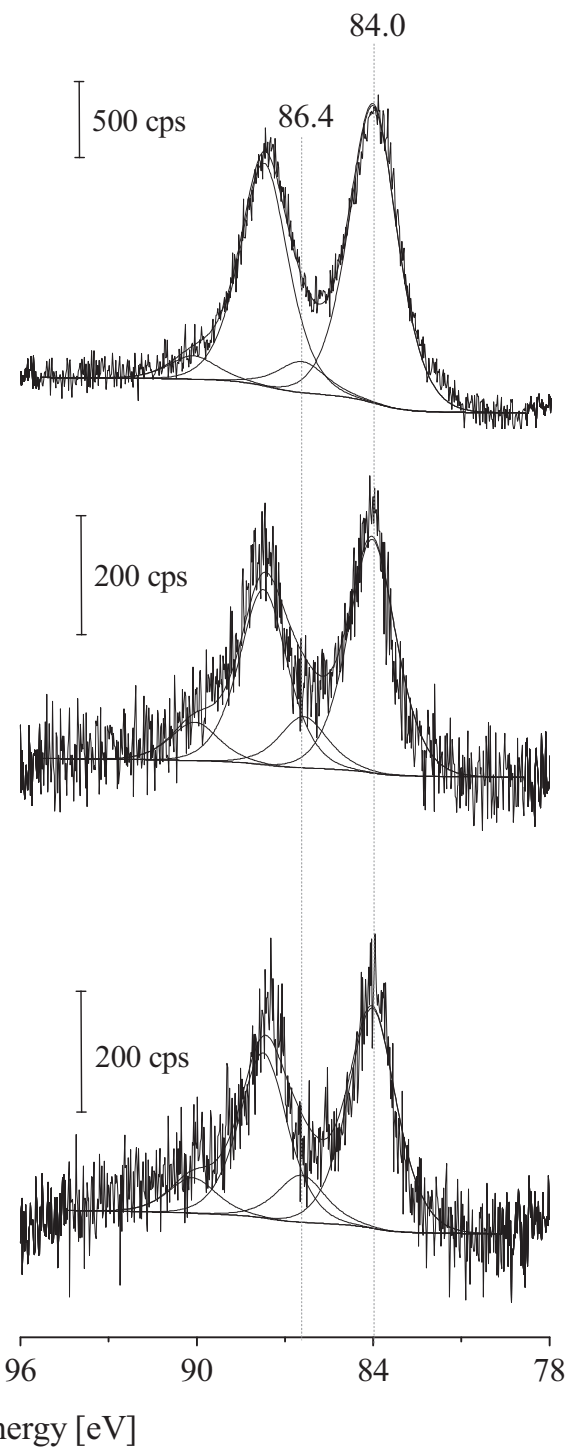


To understand the underlying changes in the atomic structure, STM measurements were also performed. First, 0.6 eqML of gold was evaporated onto titania (Fig. 44(A)). As it can be seen in the STM image of $20 \times 20 \mathrm{~nm}^{2}$, the gold clusters are well distinguishable from the titania substrate terrace and from each other. The height of the five to seven largest particles is approx. 0.5-0.7 nm, which indicates two to three atomic layers. The diameter of these $\mathrm{Au}$ nanoparticles varies between 2.5 and $3.5 \mathrm{~nm}$. Fig. 44(B) exhibits the surface morphology, which appeared after the deposition of 0.6 eqML $\mathrm{Rh}$ onto the $\mathrm{Au}$ covered surface. Although it is not possible to approach the same surface region repeatedly in our STM instrument, it was relatively easy to find regions where nearly the same amount of larger particles appeared on a $20 \times 20 \mathrm{~nm}^{2}$ area as after the $\mathrm{Au}$ deposition. This behavior indicated that the initial larger $\mathrm{Au}$ particles can be identified with high reliability. The height of the five to seven outrising particles is in the range of $0.7-0.9 \mathrm{~nm}$ with an average diameter of 3-4 $\mathrm{nm}$. Both of these latter parameters are higher by at least 10-20\% than that for the initial $\mathrm{Au}$ nanoparticles described above. This fact suggests that the footprint area of these particles increased by $20-40 \%$, while the volume increased at least by $30-50 \%$. However, by assuming a simple added Rh layer (0.6 eqML) on two to three layer high $\mathrm{Au}$ particles, the increase of the particle volume could not be more than 15-25\%. Several new particles also can be seen among the particles increased in size (Fig. 44(B)). These can be identified as $\mathrm{Rh}$ particles nucleated independently from gold on the free $\mathrm{TiO}_{2}(110)$ terraces. The size of these nanoparticles is $1.5-2.0 \mathrm{~nm}$ in diameter and $0.2-0.4 \mathrm{~nm}$ in height. For comparison, 0.6 eqML of $\mathrm{Rh}$ was also deposited on the clean $\mathrm{TiO}_{2}(110)$ surface as shown in Fig. 44(C). The average size of the Rh nanoparticles formed in this way is similar to those formed among the $\mathrm{Au}$ nanoparticles described above.

It was demonstrated that the formation of core-shell $\mathrm{Rh}-\mathrm{Au}$ particles did not depend on the state of reduction of the surface: it proceeded both on unreconstructed $\mathrm{TiO}_{2}(110)$ and also on the more reduced $\mathrm{TiO}_{2}(110)-(1 \times 2)$.

The thermodynamic driving force for $\mathrm{Au}$ atoms to assume outside positions within bimetallic clusters is due to the significantly lower surface free energy of $\mathrm{Au}$ compared to Rh [348]. When the reverse deposition sequence was applied, i.e. $\mathrm{Rh}$ was deposited on the $\mathrm{Au} / \mathrm{TiO}_{2}(110)$ surface, a very efficient place exchange between $\mathrm{Rh}$ and $\mathrm{Au}$ atoms was observed by LEIS even at room temperature. $\mathrm{Rh}$ atoms moved into the subsurface regions of bimetallic clusters and the cluster surfaces remained covered with gold. This indicates that diffusion processes within small metal clusters are rather facile compared to bulk materials.

It is a remarkable and very important observation that similar phenomena occurs on titanate nanowires (TiONW) and nanotubes (TiONT) [349] as well. The surface composition of $\mathrm{Au}-\mathrm{Rh}$ clusters on titanate nanocomposites was also investigated by LEIS. Since LEIS is extremely sensitive to the topmost layer [350,351], the results fully support the existence of the "core-shell" structure. As was observed in the case of $\mathrm{TiO}_{2}$ (110) substrate, the Rh LEIS intensity decreased dramatically with increasing gold content. The most pregnant feature was observed in the $0.5 \% \mathrm{Au}+0.5 \% \mathrm{Rh}$ case. On the monometallic systems the gold and rhodium $\mathrm{He}^{+}$scattering signals appeared at 753 and $707 \mathrm{eV}$, respectively. On bimetallic nanocomposite, however, only the gold signal showed up (Fig. 45) [181] and the rhodium peak was barely distinguisable from the noise level. LEIS experiments revealed that at the proper $\mathrm{Au}$ and $\mathrm{Rh}$ coverage the Au completely and uniformly covers the Rh nanoparticles (core-shell structure).

Although the SEM technique does not give exact quantitative comparison for all particle sizes in these cases, its results supported the main conclusion derived above. Low (1 wt \%) metal concentration was used in order to avoid the segregation of gold. Characteristic SEM images of mono- and bimetallic titanate nanowires are presented in Fig. 46. It seems conceivable that the appearance of several metallic nanoparticles in each of these very high magnification SEM images is in good agreement with the metal contents of the samples. The images were recorded right after the XPS and LEIS measurements; therefore, XPS, LEIS and SEM results can be compared directly. Metallic nanoparticles (denoted by white arrows in the figure) are clearly visible on all three samples. The average diameter of monometallic $\mathrm{Au}$ and $\mathrm{Rh}$ nanoparticles is 7.5 and $5.5 \mathrm{~nm}$, respectively. However, in the bimetallic $\mathrm{Au}+\mathrm{Rh}$ cluster the average particle diameter is around $12 \mathrm{~nm} .1-$ $1.5 \mathrm{~nm}$ gold particles (85.6 eV in XPS) are too small to be seen in these SEM images but it can be assumed that a similar increase in their volume occurs in the course of their interaction with rhodium particles, too.

On the monometallic $\mathrm{Rh} / \mathrm{TiO}_{2}$ nanowire the dominant $\mathrm{XP}$ peak for $\mathrm{Rh} 3 \mathrm{~d}_{5 / 2}$ appeared at $307.1 \mathrm{eV}$ after reduction at $573 \mathrm{~K}$ (not shown). The tailing at the higher binding energy side of this emission may be due to the original asymmetry and trace of foreign element (Mg KLL Auger peak) that remained in the sample from the preparation process. A careful deconvolution revealed an emission at $309.3 \mathrm{eV}$, presumably due to more dispersed nanoparticles. Very similar rhodium XP spectra were recorded on titanate nanotubes. In the case of the $1 \% \mathrm{Au} / \mathrm{TiO}_{2}$ nanowire two peaks were observed on the reduced sample for $\mathrm{Au} 4 \mathrm{f}_{7 / 2}$ at $83.7 \mathrm{eV}$ (metallic state) and $85.6 \mathrm{eV}$. We may attribute this feature to gold atoms in very small sized nanoparticles ("final-state" effect) [75,181] and partially oxidized state due to ion exchange process as was discussed in previous paragraph. The XP spectra of the bimetallic $\mathrm{Au}+\mathrm{Rh}$ layer supported on titania nanowires are shown in Fig. 47. Surprisingly, the emission for the higher energy peak of $\mathrm{Au} 4 \mathrm{f}_{7 / 2}$ at $85.6 \mathrm{eV}$ corresponding to the atomically dispersed state is very small in the presence of $\mathrm{Rh}$ (Fig. 47(A)), and at the same time the emission for $R h 3 d_{5 / 2}$ at around $309.3 \mathrm{eV}$ also diminished. The adsorption of $\mathrm{CO}$ at $300 \mathrm{~K}$ did not alter the position of gold emissions. The Rh XPS feature at 308.9-309.3 eV appeared or intensified a little bit after $60 \mathrm{~min} \mathrm{CO}$ adsorption may be due to the appearance of more dispersed particles (Fig. 47(B)). The CO pressure was 1.3 mbar in the preparation chamber.

The morphology of $\mathrm{Au}, \mathrm{Rh}$ and $\mathrm{Au}+\mathrm{Rh}$ supported on $\mathrm{TiO}_{2}$ nanowires and nanotubes was investigated by FTIR, employing adsorbed $\mathrm{CO}$ as a probe molecule sensitive to the local surface 

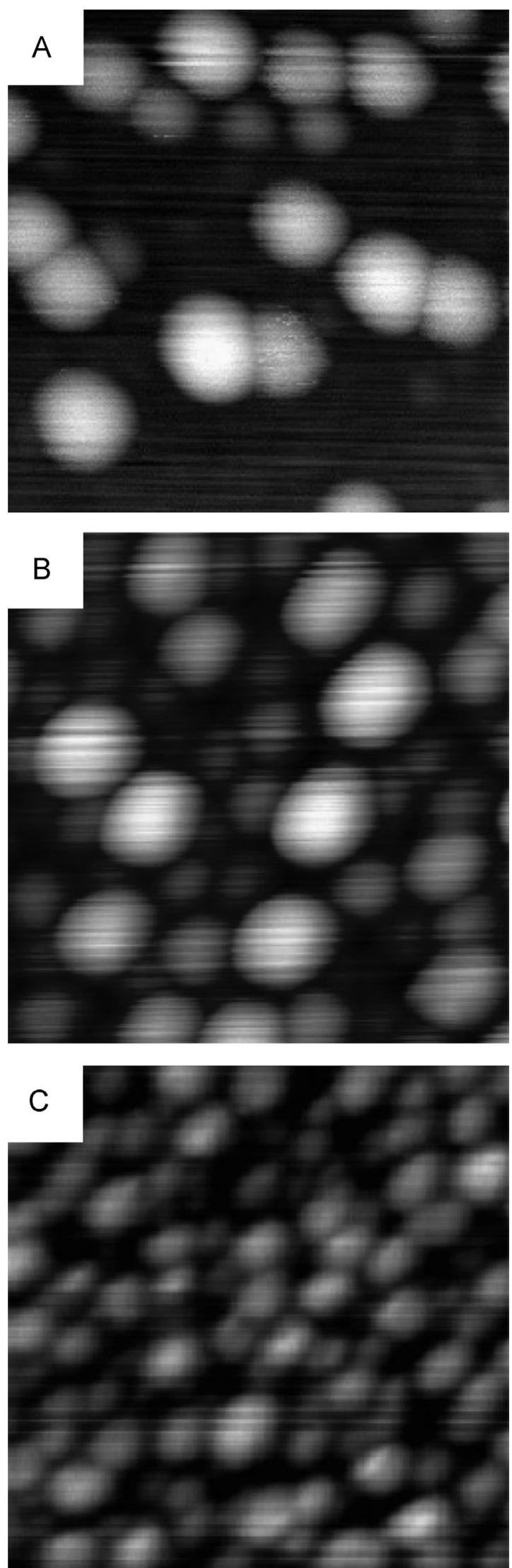

Fig. 44. Characteristic STM images of $20 \times 20 \mathrm{~nm}^{2}$ recorded on (A) a $\mathrm{TiO}_{2}(110)$ surface deposited by 0.6 eqML of Au at $320-330 \mathrm{~K}$; (B) a surface formed by postdeposition of 0.6 eqML of $\mathrm{Rh}$ on the former Au covered surface; and $(\mathrm{C})$ a $\mathrm{TiO}_{2}(110)$ surface deposited by 0.6 eqML of $\mathrm{Rh}$ at $320-330 \mathrm{~K}$. Reproduced from Ref. [346]. 


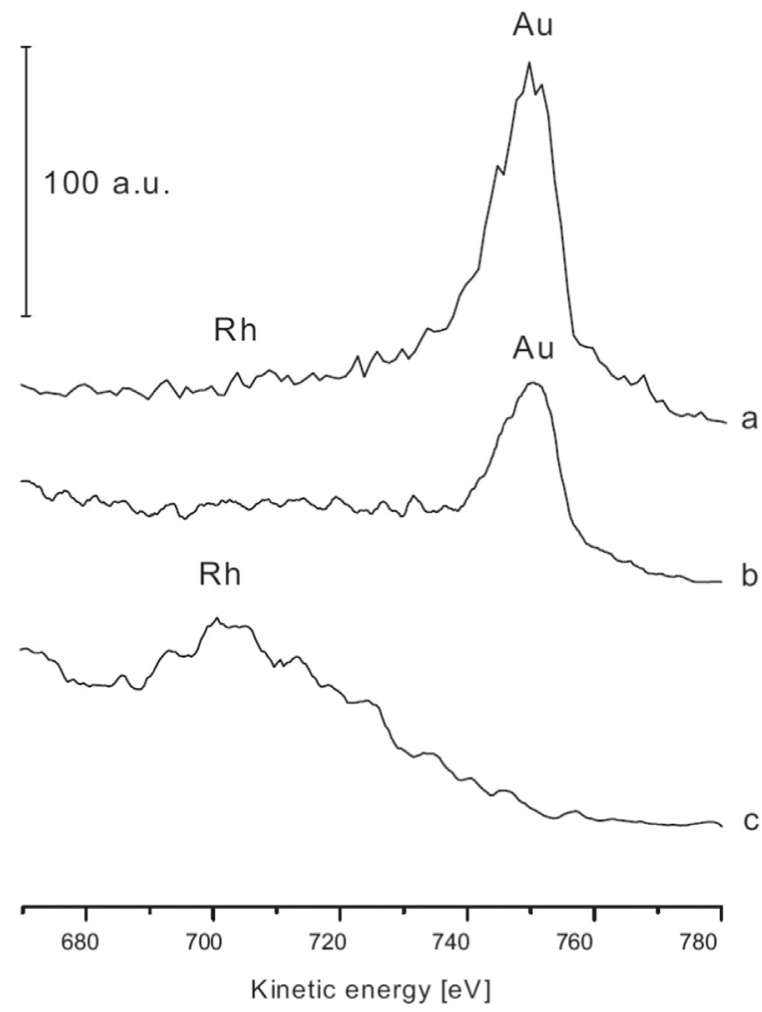

Fig. 45. LEIS spectra of $1 \% \mathrm{Au} / \mathrm{TiONW}$ (b), $1 \% \mathrm{Rh} / \mathrm{TiONW}$ (c), and $0.5 \%$ $\mathrm{Au}+0.5 \% \mathrm{Rh} / \mathrm{TiONW}$ (a). Reproduced from Ref. [349].

structure. From these IR studies we may conclude that a significant part of Rh exists in small particle sizes on both nanowires and tubes. On monometallic $\mathrm{Rh}$ supported on nanowire practically the twin form was present (2027 and $2097 \mathrm{~cm}^{-1}$ ), the linear form between these two peaks was much smaller and the bridge form was hardly observable (Fig. 48(A)). $\mathrm{CO}$ adsorption on gold supported on titania nanostructure was not observed.

It is plausible that if the gold completely and uniformly covers the $\mathrm{Rh}$ nanoparticles (core-shell structure) then adsorbed $\mathrm{CO}$ cannot be detected by FTIR. However, relatively strong $\mathrm{CO}$ bands appeared at $300 \mathrm{~K}$ at a pressure of $1.3 \mathrm{mbar}$. In the presence of gold, the peak corresponding to the linear form became stronger at $2070-2071 \mathrm{~cm}^{-1}$ and the twin CO stretching frequencies decreased (Fig. 48(B)). Apparently, there is a contradiction between the results of LEIS and CO adsorption infrared experiments. On the topmost layer there are no Rh atoms (Fig. 45) yet adsorbed CO was detected by FTIR on this surface (Fig. 48(B)). This discrepancy can be explained by a $\mathrm{CO}$ induced surface reconstruction. The adsorption of $\mathrm{CO}$ on $\mathrm{Au}-\mathrm{Rh}$ clusters may promote the diffusion of $\mathrm{Rh}$ to the surface of the cluster. Similar phenomena was observed recently in the case of bimetallic Pt-Au clusters on $\mathrm{TiO}_{2}(110)$ [352]. The same effect was also observed on $\mathrm{Pd}-\mathrm{Au}$ bimetallic model catalysts, synthesized either as thin films on Mo (110) or as nanoparticles on $\mathrm{TiO}_{2}$ thin film in $\mathrm{CO}$ oxidation at elevated (8-16 Torr) $\mathrm{CO}$ pressures [353]. Pd preferentially segregates to the surface to form contiguous $\mathrm{Pd}$ sites and $\mathrm{CO}$ oxidation reactivity is regained. The differences obtained on FTIR spectra between monometallic $\mathrm{Rh}$ and bimetallic $\mathrm{Au}-\mathrm{Rh}$
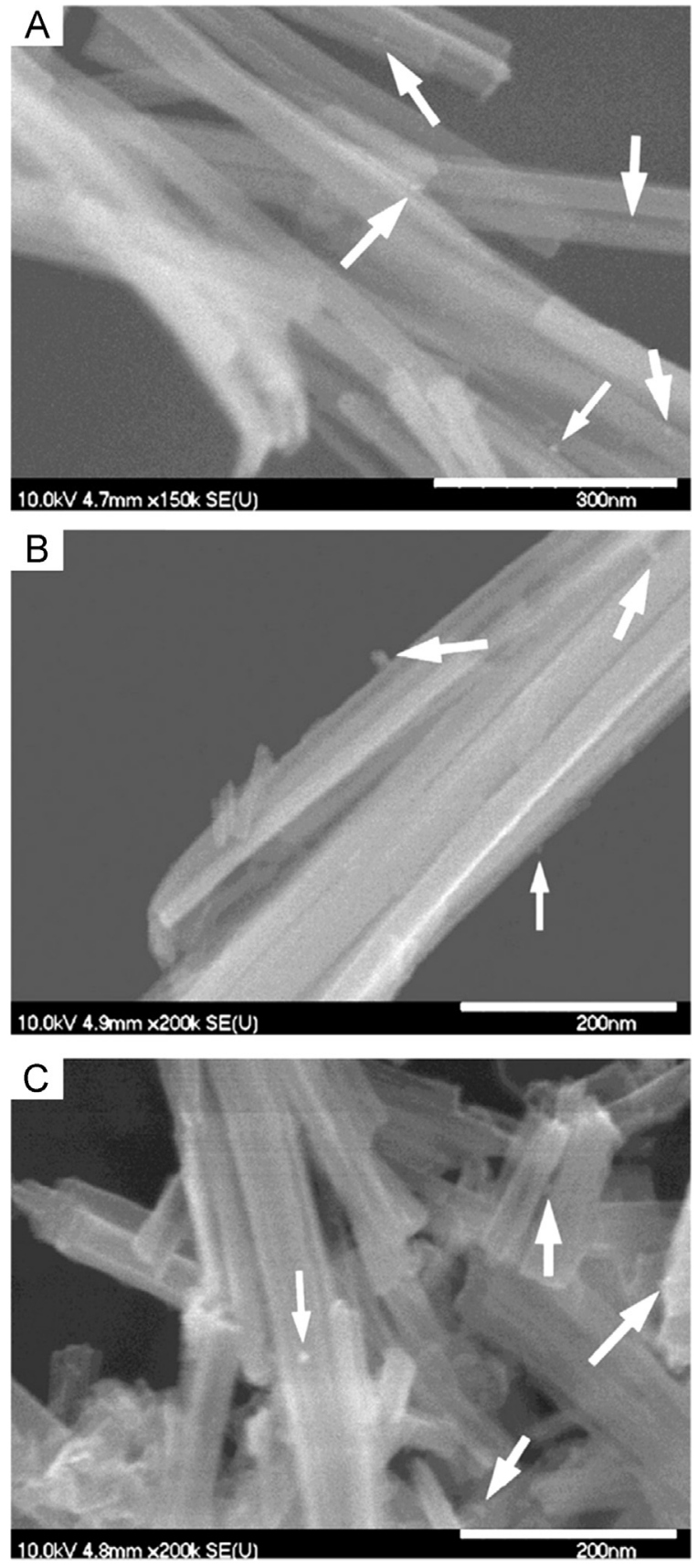

Fig. 46. Characteristic SEM images of titanate nanoeires decorated with Au (A), $\mathrm{Rh}(\mathrm{B})$ and $\mathrm{Au}+\mathrm{Rh}(\mathrm{C})$ nanoparticles. Some characteristic instances are highlighted by white arrow pointers. Reproduced from Ref. [181].

nanoclusters on titanate nanowire are not surprising. The presence of the core-shell composites and the separate highly dispersed gold clusters may significantly affect the morphology and electronic structure of $\mathrm{Rh}$ nanoparticles and therefore, the bonding modes of $\mathrm{CO}$ adsorbed on Rh sites as well. The observed FTIR feature may be related to the XPS result detected after CO adsorption. The morphology and crystallite size of segregated $\mathrm{Rh}$ could be different in the vicinity of $\mathrm{Au}-\mathrm{Rh}$ core-shell and in the inside of the core-shell structure itself.

In order to get closer to the understanding of CO-induced morphological changes, some low energy ion scattering 

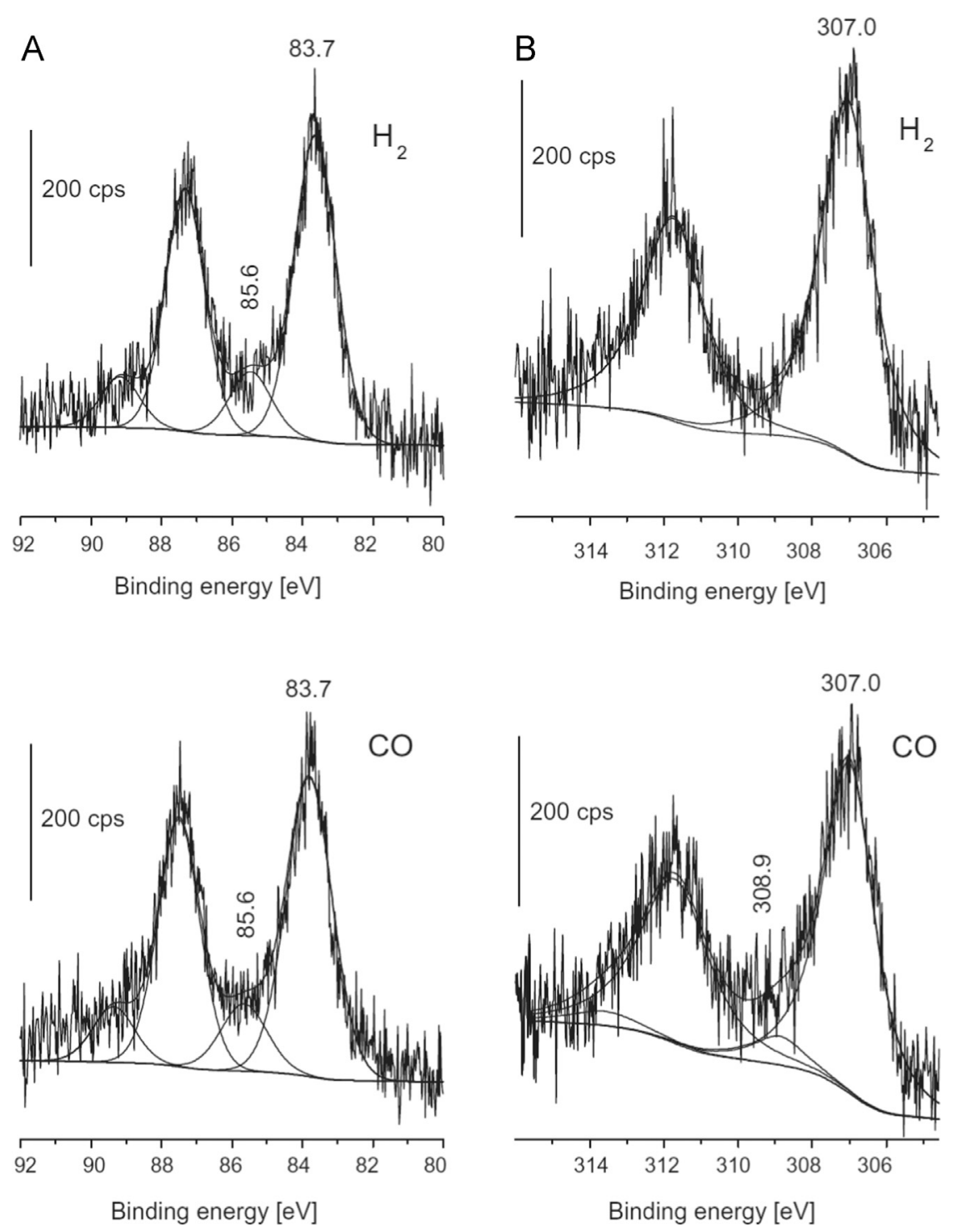

Fig. 47. XP spectra of $\mathrm{Au} 4 \mathrm{f}(\mathrm{A})$ and $\mathrm{Rh} 3 \mathrm{~d}(\mathrm{~B})$ of $0.5 \% \mathrm{Au}+0.5 \% \mathrm{Rh} / \mathrm{TiONW}$ before and after $\mathrm{CO}$ treatment. CO pressure was 1.3 mbar Reproduced from Ref. [349].

measurements (LEIS) were carried out before and after CO adsorption on $\mathrm{Au}-\mathrm{Rh}$ bimetallic clusters supported on titanate nanotubes. In these experiments two compositions were used: $0.25 \% \mathrm{Au}+0.75 \% \mathrm{Rh} / \mathrm{TiONT}$ and $0.5 \% \mathrm{Au}+0.5 \% \mathrm{Rh} /$ TiONT. The results are represented in Fig. 49. In order to avoid or minimize the sputtering effect of the $\mathrm{He}$ ions we applied a relatively low ion flux $\left(0.03 \mu \mathrm{A} / \mathrm{cm}^{2}\right)$. At the lower gold content rhodium scattering appeared at $707 \mathrm{eV}$ after hydrogen treatment at $573 \mathrm{~K}$. After $\mathrm{CO}$ adsorption at $300 \mathrm{~K}$ its intensity increased by about 20\% (Fig. 49(A)). The effect of adsorbed $\mathrm{CO}$ was more pronounced in the $0.5 \% \mathrm{Au}+0.5 \% \mathrm{Rh}$ case. Practically no or very small $\mathrm{Rh}$ scattering signal was detected indicating that the gold covered the rhodium particles (Fig. 49(B)), similarly to the nanowire case (Fig. 45). When $\mathrm{CO}$ was added to the bimetallic composite, the rhodium ion scattering signal showed up. These experimental data strongly support the above mentioned phenomena, namely that $\mathrm{CO}$ induces a reconstruction of the surface. Rh preferentially segregates to the surface to form $\mathrm{Rh}-\mathrm{CO}$ bond.

This process may imply the migration of $\mathrm{Rh}$ or $\mathrm{Au}$ atoms within the clusters or on the surface of the clusters. Considering that $\mathrm{Au}-\mathrm{Au}$ and $\mathrm{Au}-\mathrm{Rh}$ bonds are weaker than $\mathrm{Rh}-\mathrm{Rh}$ bonds, the movement of $\mathrm{Au}$ atoms may appear more probable (the shell "opens"), while Rh atoms move less. The influence of $\mathrm{CO}$ gas on this possible process is an open question. Though the $\mathrm{Au}-\mathrm{CO}$ interaction is rather weak compared to the $\mathrm{Rh}-\mathrm{CO}$ bond, the $\mathrm{CO}$ coverage on the Au capping layer is probably significant at the relatively high $\mathrm{CO}$ pressure applied. The adsorbed CO layer may decrease the energy of metal-metal bonds both for $\mathrm{Au}$ and $\mathrm{Rh}$, leading to an increased diffusion rate. It is also possible that $\mathrm{CO}$, adsorbed in some hollow site of the Au layer, can form some kind of bond with subsurface $\mathrm{Rh}$ atoms and this interaction promotes the displacement of $\mathrm{Au}$ atoms and the segregation of Rh. 

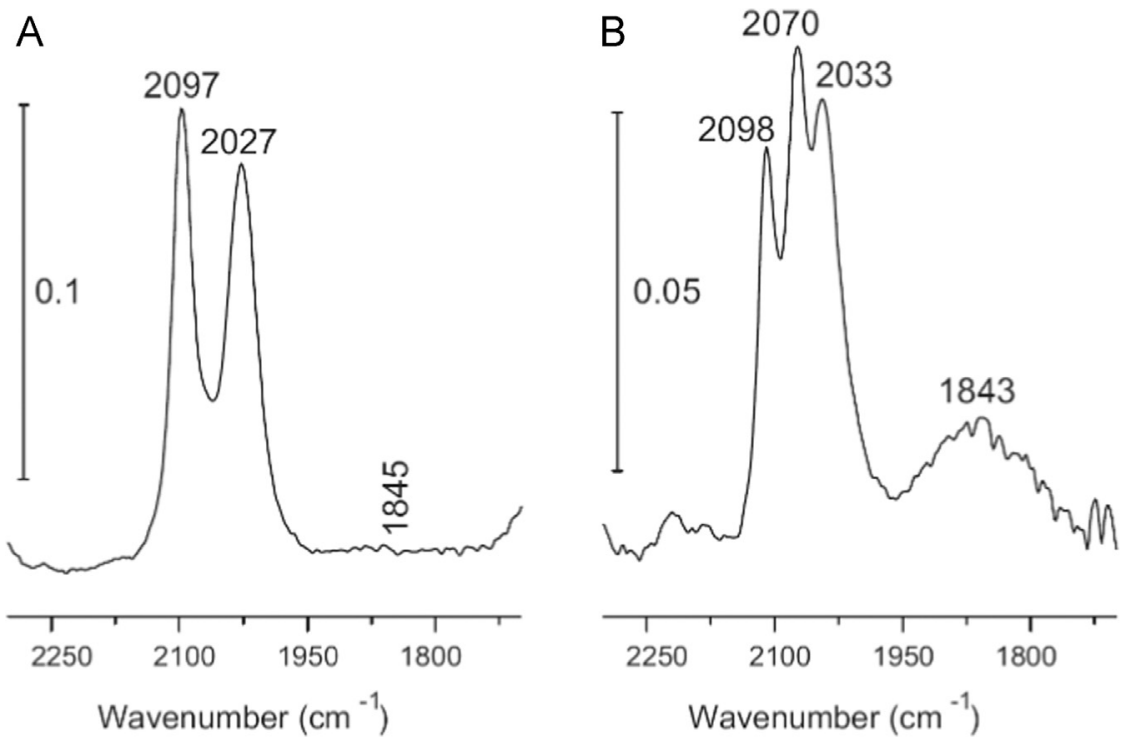

Fig. 48. FTIR spectra of adsorbed CO at $300 \mathrm{~K}$ : (A) $1 \% \mathrm{Rh} / \mathrm{TiONW}$ and (B) $0.5 \% \mathrm{Au}+0.5 \% \mathrm{Rh} / \mathrm{TiONW}$. Reproduced from Ref. [349].

Another possible scenario is that there is a continuous thermal fluctuation of metal atoms within the bimetallic clusters independent of the presence of $\mathrm{CO}$, and for short periods Rh atoms can be found on the cluster surface, which are subsequently trapped by $\mathrm{CO}$. The existence of this fluctuation may be possible, since significant movement of metal atoms within small clusters at room temperature was observed in several cases characterized by diffusion coefficients many orders of magnitude higher than the corresponding bulk values [354,355]. Anyhow, further studies are needed to understand the $\mathrm{CO}$-induced restructuring of $\mathrm{Rh}-\mathrm{Au}$ clusters in detail.

From the electrochemical point of view the properties of pure $\mathrm{Pt}, \mathrm{Au}$ and bimetallic Pt-Au alloy catalyst on titanium oxide nanotubes are all interesting. In contrast to the $\mathrm{Rh}-\mathrm{Au}$ bimetallic case, no core-shell structure forms on nanotubes, rather, alloy formation is favored. This is also the preferred case on well-ordered $\mathrm{TiO}_{2}(110)$ [356]. The electronic properties of bimetallic nanoparticles can be different from their bulk characteristics, which can lead to different alloying capabilities. Although the platinum-gold phase diagram refers to a wide miscibility gap due to limited mutual solubility of the components, the smaller nanoparticles $(d<3 \mathrm{~nm})$ do form a homogeneous alloy, because rehybridization due to band formation does not take place and all atoms retain their atomic electronic configuration [357].

Using pulse electrodeposition (PED), three kinds of nanoparticles, namely $\mathrm{Pt} /$ nanotubes, Au/nanotubes and $\mathrm{Pt}-\mathrm{Au} /$ nanotubes were deposited at three different charge densities onto highly ordered titanium dioxide nanotube (TONs) substrates. Structures, compositions, and electrocatalytic activity towards oxygen reduction and stability were investigated by SEM, XRD, EDX, ICP composition analysis and cyclic voltammetry. The stability tests indicated that $\mathrm{Pt} /$ nanotubes are more stable than $\mathrm{Pt}-\mathrm{Au} /$ nanotubes of the same deposition charge density, indicative of a better catalyst [358].
It was also found that $\mathrm{Au}-\mathrm{Pd} /$ nanotubes show higher activity in selective oxidation of alcohols than their monometallic counterparts. Bimetallic $\mathrm{Pd}-\mathrm{Au}$ supported on nanotubes exhibited higher activity than same bimetallic supported on commercial $\mathrm{TiO}_{2}$ [359]. The higher activity could be attributed to geometric and electronic effects [360].

$\mathrm{Au}, \mathrm{Rh}, \mathrm{Pd}, \mathrm{Au}-\mathrm{Rh}$ and $\mathrm{Au}-\mathrm{Pd}$ nanoparticles (NPs) were synthesized by colloidal chemical reduction and immobilized on hydrothermally-prepared rutile titania nanorods [361]. The catalysts were characterized by aberration-corrected TEM/ STEM, XPS and FTIR, and were evaluated in the hydrogenation of tetralin in the presence of $\mathrm{H}_{2} \mathrm{~S}$. Oxidizing and reducing thermal treatments were employed to remove the polyvinyl alcohol (PVA) surfactant. Reduction in $\mathrm{H}_{2}$ at $650 \mathrm{~K}$ was found efficient for removing the PVA while preserving the size (ca. $3 \mathrm{~nm}$ ), shape and bimetallic nature of the NPs. While Au-Pd NPs are alloyed at the atomic scale, Au-Rh NPs contain randomly distributed single-phase domains. Calcinationreduction of Au-Rh NPs mostly yields separated Au and Rh NPs, while pre-reduction generates a well-defined segregated structure with $\mathrm{Rh}$ located at the interface between $\mathrm{Au}$ and $\mathrm{TiO}_{2}$ and possibly present around the NPs as a thin overlayer. Both the titania support and gold increase the resistance of $\mathrm{Rh}$ and Pd to oxidation.

A novel titanate nanowire (NW)@hollow silver (Ag)/platinum $(\mathrm{Pt})$ heterostructure was fabricated by means of a simple wet chemical method. Briefly, Ag nanoparticles measuring $\sim 20 \mathrm{~nm}$ in diameter were grown onto titanate nanowires, then subsequently $\mathrm{PtO}$ shells of $\sim 1$ to $4 \mathrm{~nm}$ thickness were deposited upon the $\mathrm{Ag}$ nanoparticles to form $\mathrm{NW} @ \mathrm{Ag}-$ $\mathrm{NP} @ \mathrm{PtO}$ nanostructures. The hollow Ag-/PtOx nanostructures were created from $\mathrm{Ag}-\mathrm{NP} @ \mathrm{PtO}$ nanostructures by the outward diffusion of $\mathrm{Ag}$ from the $\mathrm{Ag}-\mathrm{NP}$ core to the $\mathrm{PtO}$ shell via $\mathrm{H}_{2} \mathrm{O}_{2}$ treatment. Finally, hollow $\mathrm{Ag} / \mathrm{Pt}$ heterostructures of approximately $20 \mathrm{~nm}$ diameter were synthesized by the reduction of the hollow Ag-/PtOx nanostructures. Given the 
A

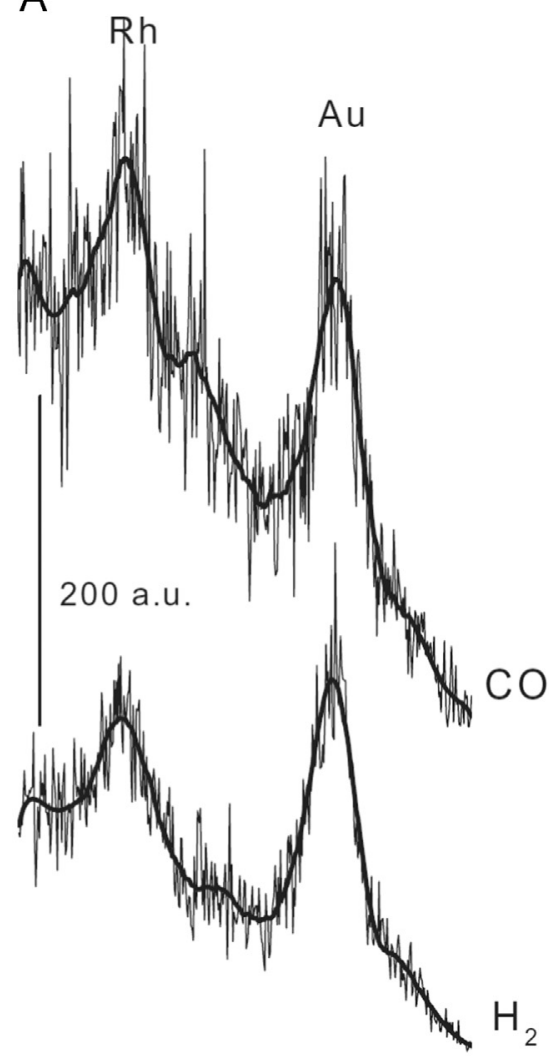

$\begin{array}{llllll}680 & 700 & 720 & 740 & 760 & 780\end{array}$

Kinetic energy $[\mathrm{eV}]$
B

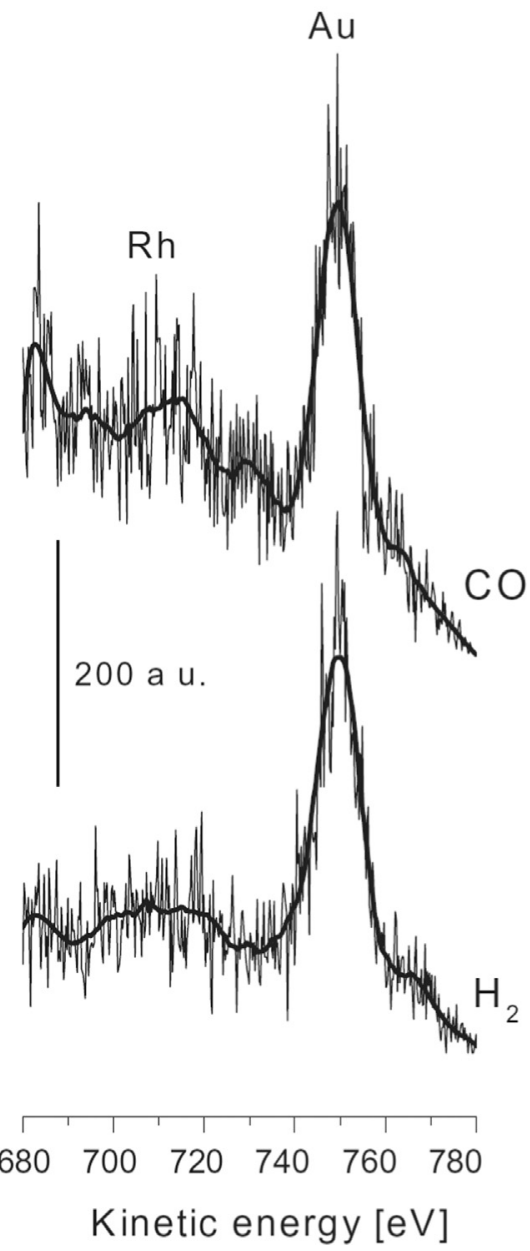

Fig. 49. LEIS spectra of $0.25 \% \mathrm{Au}+0.75 \% \mathrm{Rh} / \mathrm{TiONT}(\mathrm{A}), 0.5 \% \mathrm{Au}+0.5 \% \mathrm{Rh} / \mathrm{TiONT}$ (B) before and after $\mathrm{CO}$ adsorption at $300 \mathrm{~K}$. The $\mathrm{CO}$ pressure was 1.3 mbar. Reproduced from Ref. [349].

accessible interfaces of bimetallic Ag/Pt shell, the catalytic efficiency of the titanate NW@hollow Ag/Pt heterostructures was significantly enhanced, which was confirmed by the hydrogenation of p-nitrophenol into p-aminophenol with $\mathrm{NaBH}_{4}$ as reducing agent in aqueous phase [362]. The schematic illustration of the formation mechanism of $\mathrm{Ag} / \mathrm{Pt}$ heterostructures can be seen in Fig. 50.

In summary, when comparing different bimetallic nanoclusters on titanates (mainly on titanate nanotubes) one should consider that $\mathrm{Rh}+\mathrm{Au}$ and $\mathrm{Ag}+\mathrm{Pt}$ form mainly core-shell structure and separated $\mathrm{Au}$ and $\mathrm{Rh}$ clusters, whereas in the $\mathrm{Au}+\mathrm{Pd}$ and $\mathrm{Au}+\mathrm{Pt}$ bimetallic systems alloy formation is favored.

\subsection{Phase stability and phase transformation of titanate nanostructures upon metal loading}

In the previous sections it was pointed out that the $\mathrm{H}$-form nanowires preserve the wire-like morphology during the heat treatment up to $873 \mathrm{~K}$. However, more and more textural discontinuities can be observed as the temperature is increased. The holey structure can be attributed to the continuous transformation of protonated titanate nanostructures to $\mathrm{TiO}_{2}$ (anatase) followed by water formation and release from the structure. This process resulted in the rearrangement of the formed anatase crystals and the appearance of unfilled spaces in the structure of the whole nanowire.

TEM images demonstrated the tubular morphology of the as-synthesized titanate nanotubes with a diameter of $\sim 7 \mathrm{~nm}$ and length up to $80 \mathrm{~nm}$. The acidic washing process resulted in a mild destruction of the inner and outer walls of the nanotubes. In correlation with the XRD and Raman results no morphological degradation after heat treatment up to $573 \mathrm{~K}$ could be observed. At higher temperature the tubular structure started to collapse and transform into rod-like nanostructures. At $873 \mathrm{~K}$ the tubular morphology totally collapsed that resulted in short nanorods and $\mathrm{TiO}_{2}$ nanoparticles with an average size of $\sim 10 \mathrm{~nm}$.

The morphology of pristine nanotubes was checked by scanning electron microscopy. The preparation of supported nanotubes began with the dissolution of $4 \mathrm{mg}$ nanotube powder in $150 \mathrm{ml}$ distiller water followed by ultrasonic treatment for $15 \mathrm{~min}$. Subsequently, one drop by a precision pipette was dropped on a sample surface cleaned by annealing and Ar ion bombardment in UHV and taken out just before the procedure. The drop covered almost the full area of the sample 


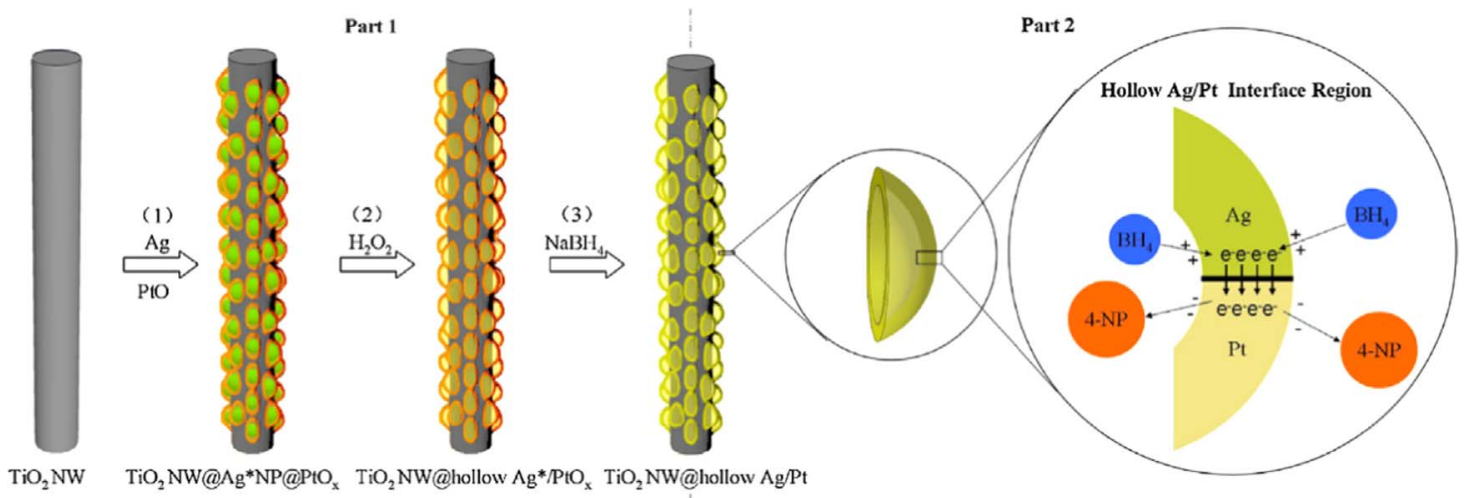

Fig. 50. Schematic illustration of the formation mechanism of titanate NW@hollow Ag/Pt heterostructures. Reproduced from Ref. [362].
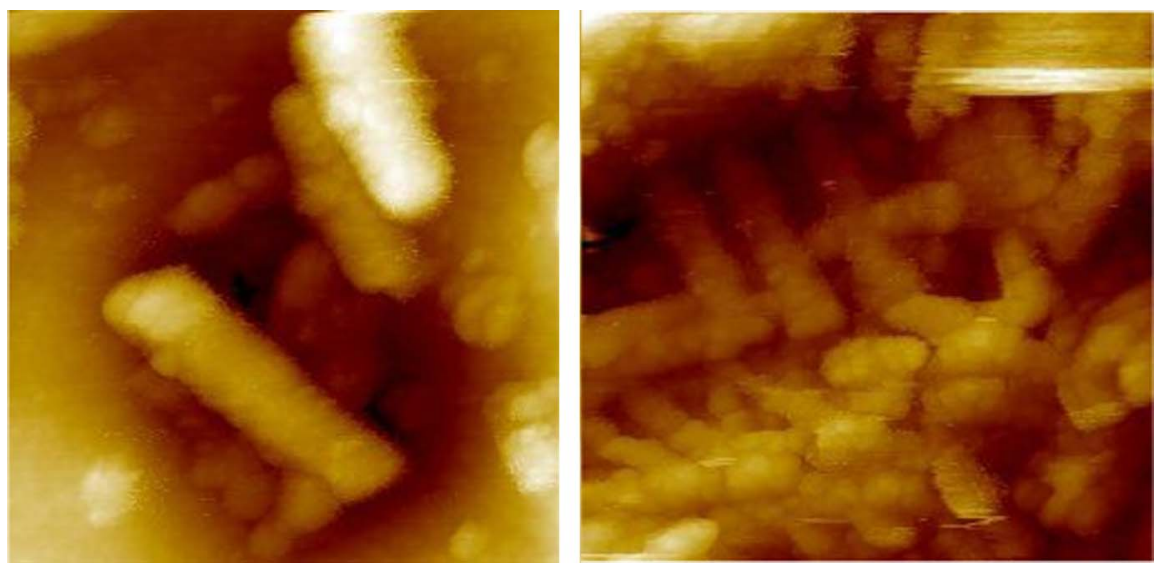

Fig. 51. STM images $\left(100 \times 100 \mathrm{~nm}^{2}\right)$ of titanate nanotubes recorded on $\mathrm{Cu}(111)$ (left) and on $\mathrm{TiO}_{2}(110)$ (right), respectively (Berkó et al., unpublished).

(if not, a second drop was applied immediately after the first one) and the drying process took only a few minutes at room temperature in air. The successful production of a homogeneous covering layer was visible by eyes. $\mathrm{Cu}(110)$ and $\mathrm{TiO}_{2}(110)$ were used as substrate (Fig. 51).

The chemical cleanness of the substrate surfaces - $\mathrm{Cu}(110)$ and $\mathrm{TiO}_{2}(110)$ single crystals - was checked in UHV by Augerelectron spectroscopy (AES) and STM (Fig. 51). After the preparation of the nanotube layer, some carbon contamination was always observed. This surface carbon disappeared only in the temperature range $(573-600 \mathrm{~K})$ where the decomposition also started. Therefore, it was concluded that the surface of the $\mathrm{TiO}_{2}$ nanotubes binds carboneous species in air.

The stability of the wire-like or tubular morphology can be affected by adatoms. The effects of $\mathrm{Na}, \mathrm{Ag}, \mathrm{Rh}$ and $\mathrm{Au}$ adatoms on the stability and phase transformation of titanate nanostructures are discussed below, as these are considered as crucial issues in various applications in the sensor, catalytic and photocatalytic fields.

The influence of sodium on the textural, structural, morphological and photocatalytic properties of titanate nanotubes calcined at different temperatures has been extensively studied [363]. The characterization results clearly emphasize the role of sodium in structural and morphological transformations. First of all, TEM studies revealed that the presence of sodium allows maintaining the nanotubular morphology up to $800 \mathrm{~K}$ vs. $700 \mathrm{~K}$ in the absence of sodium.

The TGA curves of the H-TNT and Na-TNT materials are reported in Fig. 52(A). Since only water is expected to be lost during the thermogravimetric analysis, results clearly show that high amount of water was initially present in both samples. This percentage is however higher on the sodium-free H-TNT sample with a loss of $19.4 \mathrm{wt} \%$ up to $600{ }^{\circ} \mathrm{C}$ vs. $14.8 \mathrm{wt} \%$ for Na-TNT. This result is in agreement with preceding Raman results showing higher water content on titanate samples when sodium is completely removed.

XRD and Raman spectroscopy also show that $\mathrm{Na}$ adatoms increase the stability of nanotubes by $100{ }^{\circ} \mathrm{C}$. The Raman profile changes very little after the elimination of sodium and shows the characteristic bands of the titanate phase (Fig. 53 (A)). However, a slight red-shift of the bands now at $271 \mathrm{~cm}^{-1}$ $\left(-6 \mathrm{~cm}^{-1}\right.$ vs. Na-TNT) and at $285 \mathrm{~cm}^{-1}\left(-5 \mathrm{~cm}^{-1}\right)$ is observed. Bands were also observed at $642 \mathrm{~cm}^{-1}$ and at $674 \mathrm{~cm}^{-1}$ corresponding to a stronger red-shift of $35 \mathrm{~cm}^{-1}$. Similar shifts were also observed by Kim et al. [364]. However, the bands initially present at $390 \mathrm{~cm}^{-1}$ and $450 \mathrm{~cm}^{-1}$ blue shift to $395 \mathrm{~cm}^{-1}$ and $454 \mathrm{~cm}^{-1}$ after the removal of sodium, but only to a lower extent suggesting their weak sensitivity to the presence of sodium. Moreover, the 

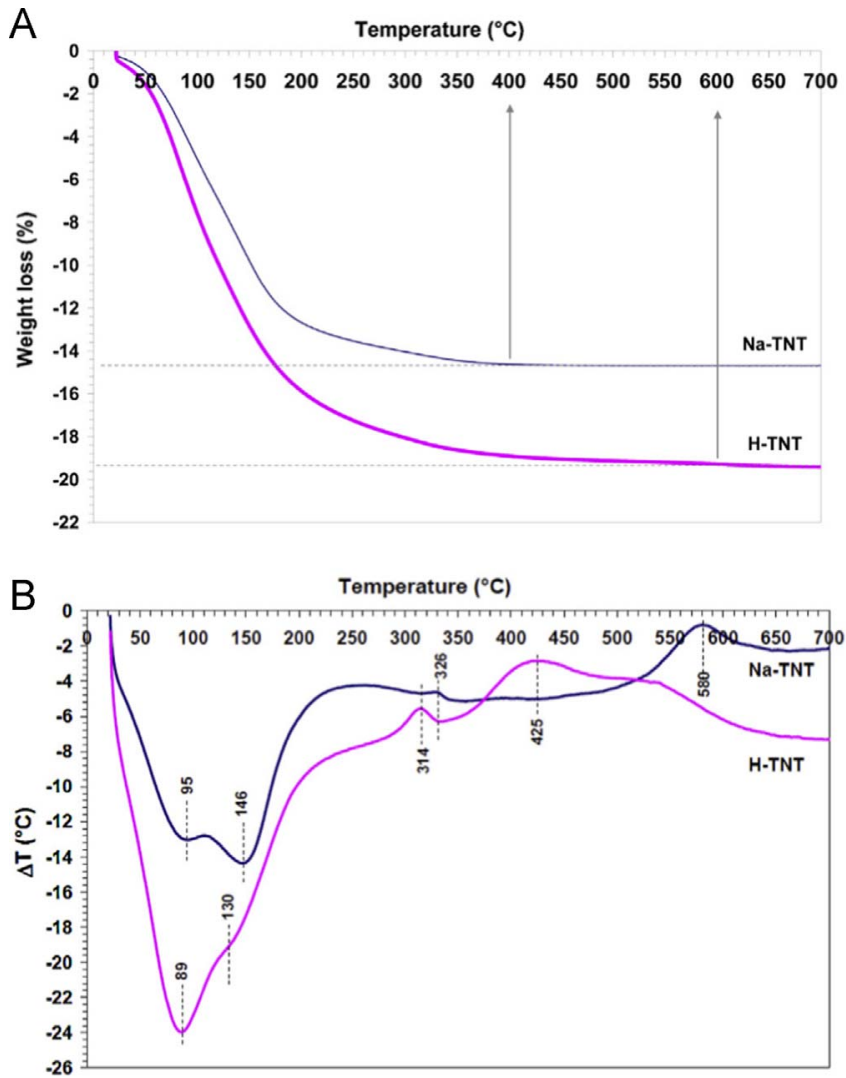

Fig. 52. TGA (A) and DTG (B) curves of Na-TNT and H-TNT. Reproduced from Ref. [363].

bands in the $550-750 \mathrm{~cm}^{-1}$ range assigned to covalent Ti-O$\mathrm{H}$ bonds tend to become broader in the H-TNT sample. This broadening tendency can be assigned to higher water content in the H-TNT nanotubular titanate structure [363].

Results show that the prepared nanotubes contain considerable amounts of water and consist of titanates with an orthorhombic structure and formulas of $\mathrm{Na}_{2-x} \mathrm{H}_{x} \mathrm{Ti}_{2} \mathrm{O}_{5} \cdot n \mathrm{H}_{2} \mathrm{O}$ and $\mathrm{H}_{2} \mathrm{Ti}_{2} \mathrm{O}_{5} \cdot n \mathrm{H}_{2} \mathrm{O}$, where $x$ depends on the acid washing process. Phase structure, morphology, specific surface area and pore size distribution strongly depend on the sodium content and the calcination temperature. Results demonstrate that sodium mainly influences the structural transformation of titanate into anatase through a slower dehydration process that shifts the transformation of titanate into anatase to a higher temperature [363]. However, the presence of sodium is not a pre-requisite for preserving the nanotubular morphology. Indeed, the end of the dehydration process corresponds neither to the formation of only anatase nor to the breaking of the nanotubes. Sodium also hinders the formation of pure anatase samples since residual sodium coming from the initial titanate phase tends to readily react with anatase to form a hexatitanate phase. These structural differences influence the photocatalytic activity of titanate nanotubes [363].

Alkali metal $\left(\mathrm{Na}^{+}, \mathrm{K}^{+}, \mathrm{Li}^{+}\right)$intercalated titanate nanotubes were studied by vibrational spectroscopy (Raman and FT-IR), X-ray diffraction and electron microscopy. The vibrational spectroscopic data shown that the most affected mode is
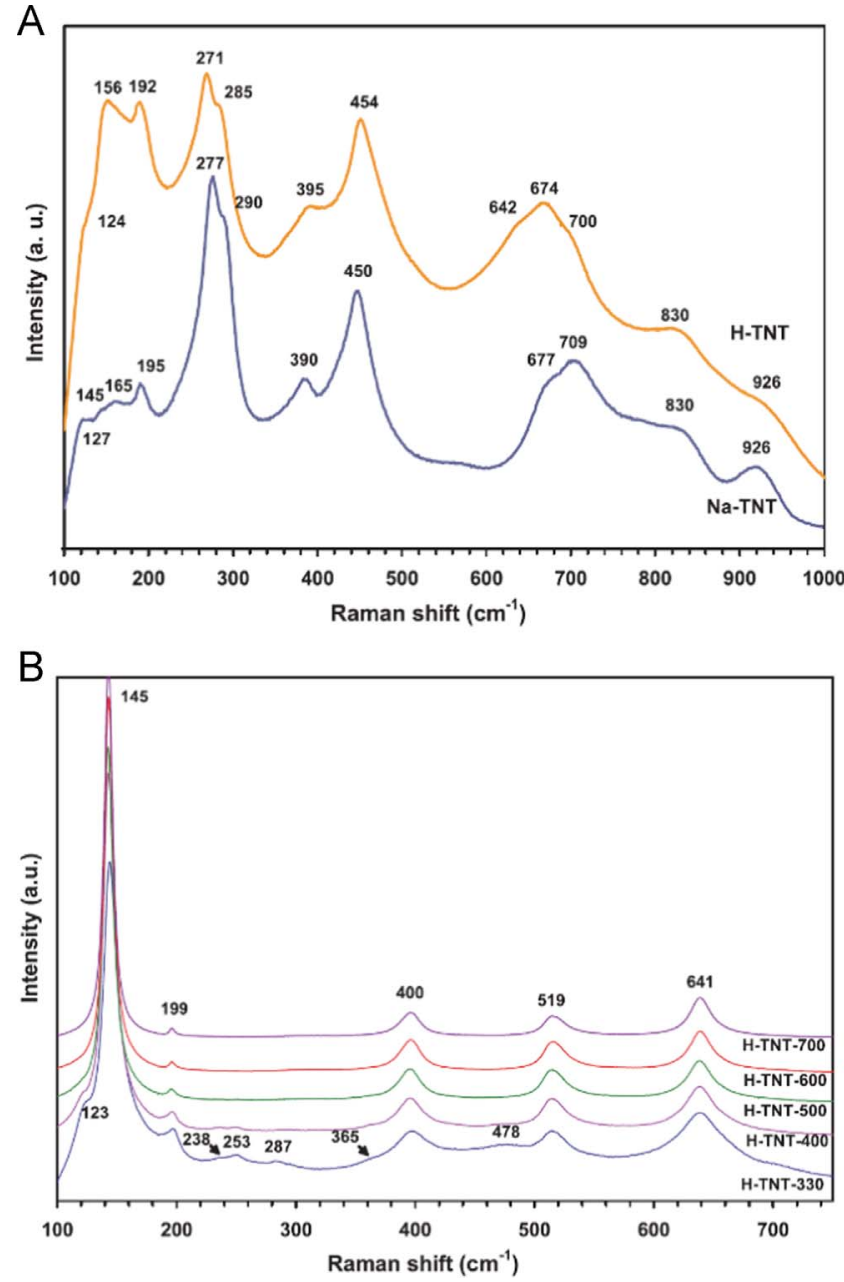

Fig. 53. Raman spectra of the Na-TNT and H-TNT samples before calcination (A) and of the H-NTN samples after calcination at different temperatures. Reproduced from Ref. [363].

that related to the $\mathrm{Ti}-\mathrm{O}$ bond whose oxygen is not shared among the $\mathrm{TiO}_{6}$ units of the framework structure [365].

The transformation of high surface area titanate nanotubes, either in the hydrogen or in the silver exchanged form, into $\mathrm{TiO}_{2}$ nanocrystals (anatase) has been studied by SEM, TEM, AFM, and $\mathrm{N}_{2}$ adsorption [233]. The thermal treatment of hydrogen titanate nanotubes at $800 \mathrm{~K}$ leads to the formation of regularly arranged crystalline $\mathrm{TiO}_{2}$ anatase nanocrystals originating from the fragmentation of nanotubes, as was established in other works.

In silver loaded titanates, the presence of $\mathrm{Ag}^{+}$species (that convert to $\mathrm{Ag}^{0}$ upon thermal treatment), plays a key role in promoting the formation of anatase nanocrystals. The presence of silver reduces the specific surface area and increases the degree of crystallinity of the titanate nanoparticles. A possible explanation concerning the role of $\mathrm{Ag}^{+}$in enhancing the crystallinity of the sample could be related to its activity in suppressing the formation of $\mathrm{Ti}^{3+}$ species [366].

$\mathrm{Rh}$-induced support transformation phenomena in titanate nanowires and nanotubes were studied with Raman spectroscopy, XRD and HRTEM in detail [183]. Fig. 54 presents the Raman spectra of Rh loaded titanate nanotubes and nanowires, respectively, as a function of heat treatment temperature. For 

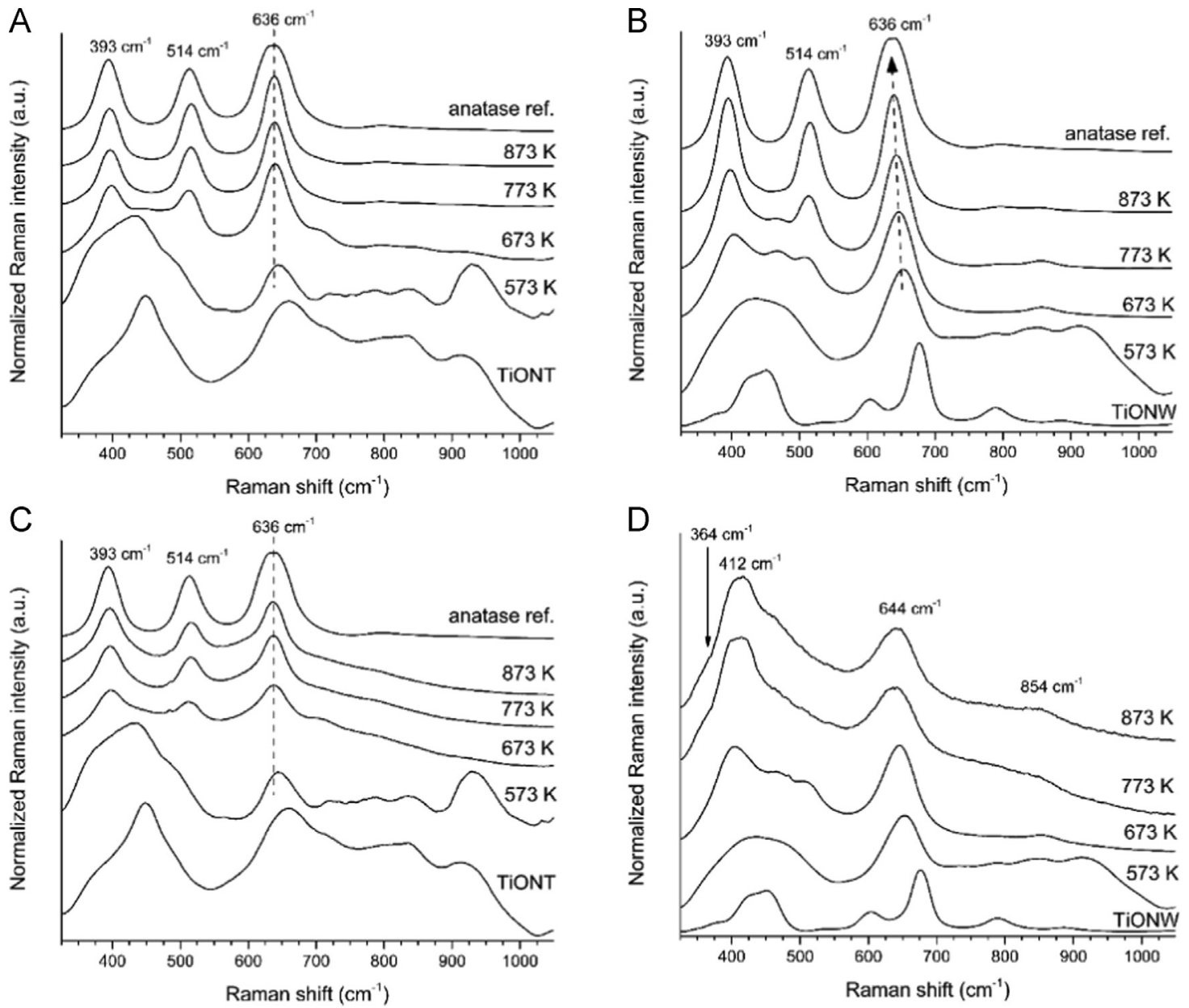

Fig. 54. Normalized Raman spectra of the thermal behavior of trititanate nanotubes (A) and nanowires (B). The spectra of as-synthesized samples are depicted at the bottom and the spectrum of a commercial anatase reference sample is shown at the top of each graph. Graphs (C) and (D) illustrate the thermal behavior of Rh loaded trititanate nanotubes and nanowires, respectively. Reproduced from Ref. [183].

comparison the spectra of the pristine and heat treated titanate nanotubes (Fig. 54(A)) and nanowires (Fig. 54(B)) are also shown. The final product of the transformation of Rh loaded nanotubes features the characteristic anatase peaks at 393,514 and $636 \mathrm{~cm}^{-1}$ assigned to the $\mathrm{B}_{1 \mathrm{~g}}, \mathrm{~A}_{1 \mathrm{~g}}$ and $\mathrm{E}_{2 \mathrm{~g}}$ modes, respectively (Fig. 54(C)). On the other hand, the Raman spectrum of the recrystallized $\mathrm{Rh}$ containing titanate nanowires lacks these anatase signals. The spectral series in Fig. 54(D) illustrates the temperature evolution of a shoulder at $364 \mathrm{~cm}^{-1}$, an envelope of overlapping peaks centered at $412 \mathrm{~cm}^{-1}$, a broad peak centered at $644 \mathrm{~cm}^{-1}$ and a very broad peak at $854 \mathrm{~cm}^{-1}$. A similar Raman spectrum was recently identified as that of a $\beta-\mathrm{TiO}_{2}$ phase by Wang et al. [367]. Therefore, we conclude that (i) the $873 \mathrm{~K}$ heat treatment of $\mathrm{Rh}$ loaded titanate nanotubes and nanowires yields products of different phase structure, (ii) Rh loaded titanate nanotubes transform into anatase under the studied experimental conditions and (iii) the spectral features of the transformation product of $\mathrm{Rh}$ loaded titanate nanowires can be adequately explained by assuming that this product exhibits the $\beta-\mathrm{TiO}_{2}$ structure [183].

The phase transformation to anatase occurs $100 \mathrm{~K}$ lower then in the case of metal-free titanate nanotubes. It appears that the $\mathrm{Rh}$ adatom has the same catalytic effect on the transformation as silver does [233].

$\mathrm{Rh}$ decorated nanowires transform into the $\beta-\mathrm{TiO}_{2}$ structure (Fig. 55(A)) as opposed to their rhodium-free counterparts' recrystallization to anatase. The formation of the $\beta-\mathrm{TiO}_{2}$ structure is indicated by the appearance of XRD peaks with Miller indices of (200), (110), (002) (111), (003), (020), (022), (711), (313), (023) and (712) at $15.4^{\circ}, 24.9^{\circ}, 28.6^{\circ}, 29.4^{\circ}$, $43.5^{\circ}, 48.5^{\circ}, 57.3^{\circ}, 58.3^{\circ}, 61.7^{\circ}, 68.2^{\circ}$ and $76.8^{\circ}$, respectively. Full width at half maximum (FWHM) of the dominant reflections attributed to anatase $25.3^{\circ}(101)$ and $\beta-\mathrm{TiO}_{2} 24.9^{\circ}$ (110) in case of pristine and $\mathrm{Rh}$ decorated titanate nanowires, respectively, indicate the degree of crystallinity at various heat treatment temperatures. Upto $673 \mathrm{~K}$ the crystallinity of the samples did not improve as indicated by the constant (Rh decorated nanowires) or increasing (pristine nanowires) FWHM values. This moderate crystal amorphization can be assigned to sample drying and structural water loss. The subsequent recrystallization processes resulting in a coherent system of nanopartcles were preferential in the case of pristine nanowires. At higher temperatures fusion of the nanoparticles becomes favorable and this resulted in lower FWHM values. 

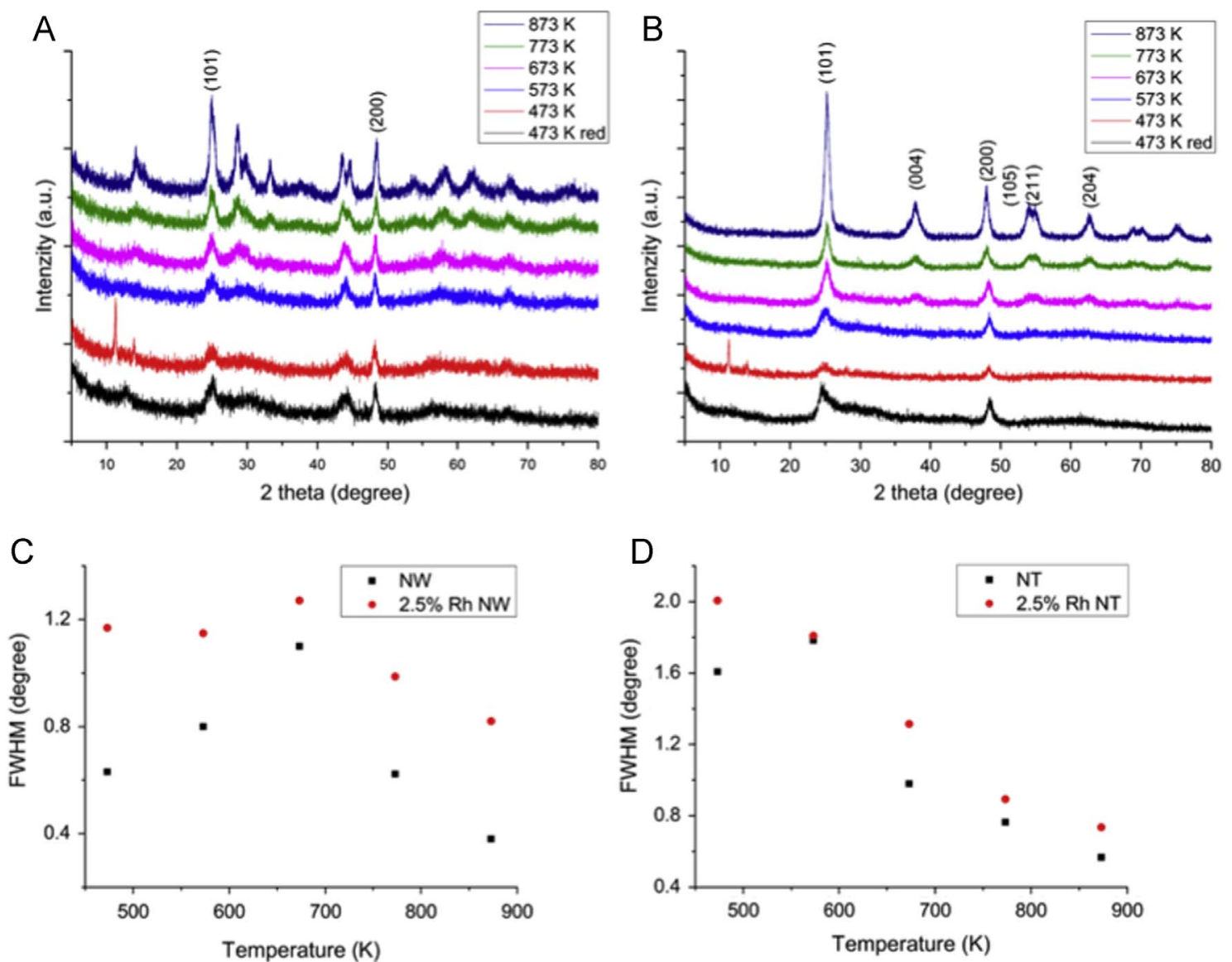

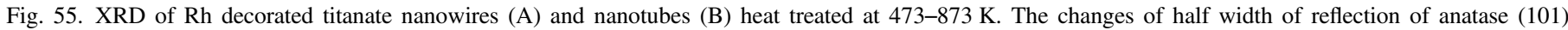
reflection at $25.3^{\circ}$ and the $\beta-\mathrm{TiO}_{2}(110)$ reflection at $24.9^{\circ}$ during heat treatment are also displayed. Reproduced from Ref. [183].

Anatase formation dominated the thermal annealing process in both of acid treated and $\mathrm{Rh}$ decorated nanotubes (Fig. 55 (B)) as indicated by the appearance of anatase reflections (101), (004), (200), (105), (211) and (204) at 25.3, $37.8^{\circ}$, $48.1^{\circ}, 53.9^{\circ}, 55.1^{\circ}$ and $62.4^{\circ}$. The FHWM of the most intensive reflection of anatase $(101)$ at $25.3^{\circ}$ indicated that the crystal structure is stable below $673 \mathrm{~K}$ compared to the nanowire form and started to improve at elevated temperatures in accordance with TEM and XRD results. The decreasing FWHM shows the increasing degree of crystallinity of both unmodified and $\mathrm{Rh}$ decorated titanate nanotubes at elevated temperatures ( > $573 \mathrm{~K}$ ) [183].

The influence of gold additives on the stability and phase transformation of titanate nanostructures was studied by Raman spectroscopy, XRD and HRTEM [52]. Fig. 56 (C) presents the Raman spectra of $2.5 \mathrm{wt} \%$ Au loaded titanate nanotubes as a function of heat treatment temperature. Raman spectra of acid washed $\mathrm{H}$-form titanate nanotubes and nanowires are presented in Fig. 56(A) and (B) together with the spectrum of a reference anatase sample for comparison. Spectral features related to Au loaded nanotubes start transforming into anatase characteristics immediately as heat treatment commences. The characteristic anatase peaks at 393, 514 and $636 \mathrm{~cm}^{-1}$ (assigned to the $\mathrm{B}_{1 \mathrm{~g}}, \mathrm{~A}_{1 \mathrm{~g}}$ and $\mathrm{E}_{2 \mathrm{~g}}$ modes, respectively) appeared already at $473 \mathrm{~K}$. This shows that gold catalyses the transformation of the titanate nanotube structure to anatase. It seems that this catalytic ability of $\mathrm{Au}$ is somewhat higher than that of $\mathrm{Rh}$ under the same experimental conditions. In the case of $\mathrm{Rh}$ adatoms a clear anatase phase could be identified at $673 \mathrm{~K}$ in the Raman spectra.

It is interesting that the destroyed nanotubes can be regenerated by $\mathrm{Cd}(\mathrm{II})$ ions [368]. This was investigated by cycled $\mathrm{Cd}$ (II) adsorption and desorption processes. The virgin TNTs, absorbed TNTs, desorbed TNTs and regenerated TNTs were systematically characterized. The ion-exchange mechanism with $\mathrm{Na}^{+}$in TNTs was confirmed by FTIR spectroscopy. The recovery of the damaged tubular structures was revealed by TEM and XRD. It was ascribed to the asymmetric distribution of $\mathrm{H}^{+}$and $\mathrm{Na}^{+}$on the surface side and interlayer region of TNTs. More importantly, the cost-effective regeneration was found to be possibly related to a complex form of TNTs-OCd $+\mathrm{OH}^{-}$identified by XPS.

A markedly different Raman spectral feature was observed in the case of Au loaded titanate nanowires (Fig. $56(\mathrm{D}))$. The spectra of samples heat treated between $473 \mathrm{~K}$ and $873 \mathrm{~K}$ are very similar and do not indicate any anatase formation. The main differences between these spectra and that of the unannealed titanate nanowires are the general line broadening and (i) the downshift of the $676 \mathrm{~cm}^{-1} \mathrm{E}_{2 \mathrm{~g}}$ mode to $640 \mathrm{~cm}^{-1}$, (ii) the broadening of the $600 \mathrm{~cm}^{-1}$ peak, and 

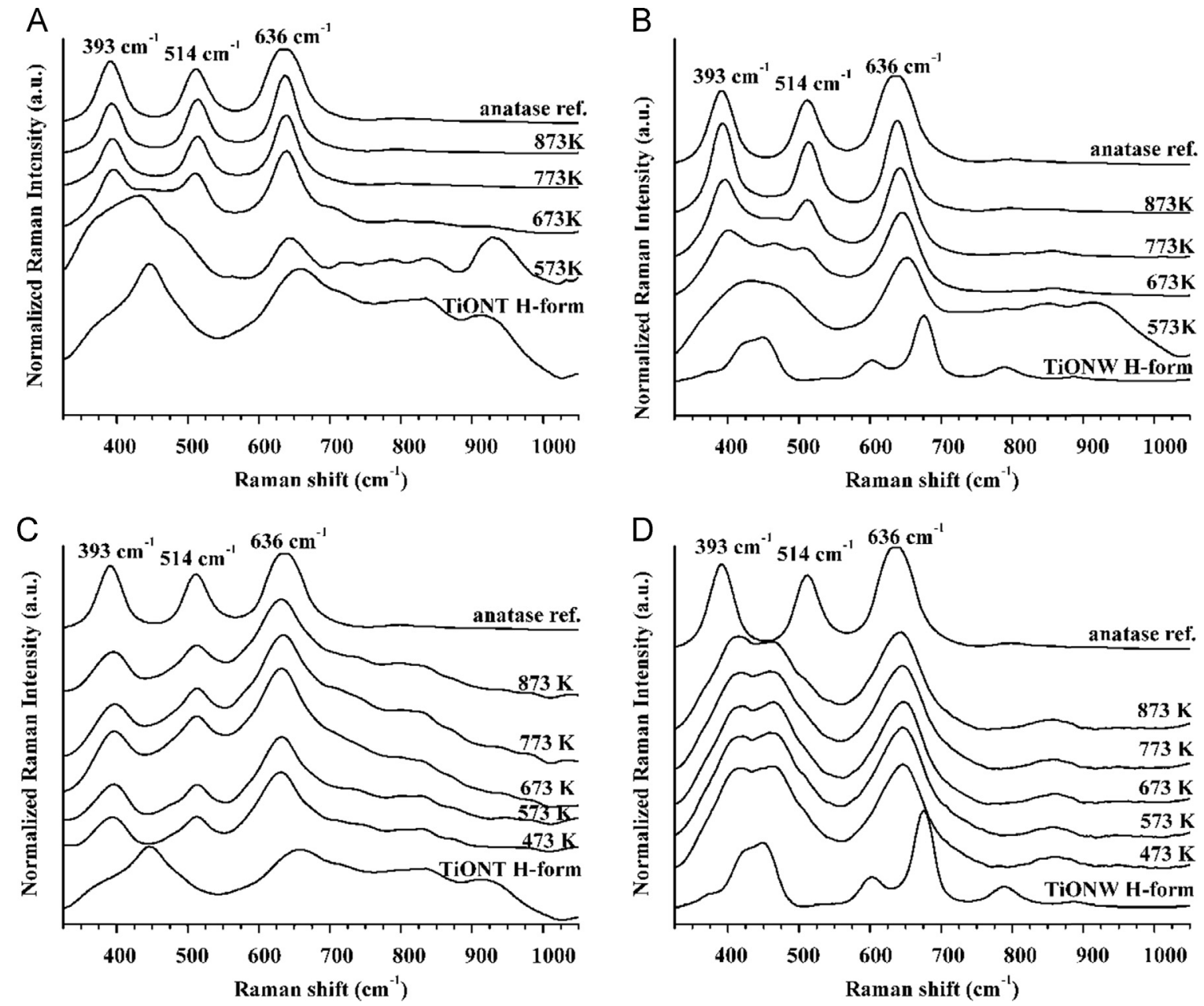

Fig. 56. Normalized Raman spectra of the thermal behavior of H-form titanate nanotubes (A) and nanowires (B). Graphs (C) and (D) illustrate the thermal behavior of Au loaded titanate nanotubes and wires, respectively. The commercial anatase reference sample is shown at the top of each spectrum. Reproduced from Ref. [52].

(iii) the simultaneous red- and blue-shift of the $424 \mathrm{~cm}^{-1}$ and $449 \mathrm{~cm}^{-1}$ peaks to 410 and $460 \mathrm{~cm}^{-1}$, respectively. The resulting spectrum still resembles a layered titanium oxide material but it is certainly different from that of the original titanate nanowires. Thomas and Yoon [369] have reported a very similar spectrum for gold decorated titanate nanofibers and identified the material as $\beta-\mathrm{TiO}_{2}$. That phase is present in the nanowires right from the beginning of the hydrothermal synthesis, but its extent appears to have been increased by the gold-catalyzed transformation of the titanate phase.

The effect of gold additive on the phase stability was also investigated by XRD [52]. For comparison the structure and thermal behavior of gold-free titanate nanostructures were also characterized by X-ray diffraction. As XRD patterns show (Fig. 57(A)), the acidic treatment resulted in the degradation of the initial crystal structure of titanate nanotubes, which manifested in the disappearance of the reflection characteristic of the tubular interlayer distance $\left(2 \Theta=\sim 10^{\circ}\right)$. Protonation also induced the transformation of titanate nanostructures to anatase form [183].

When the H-form titanate nanotubes were decorated with $2.5 \mathrm{wt} \%$ gold adatom, anatase reflections appeared already at
$473 \mathrm{~K}$ indicating that the transformation from the trititanate to anatase is very advantageous (Fig. 57(B)). For comparison it is worth mentioning that gold is a better catalyst for this transformation than rhodium [183]. Moreover, the presence of sodium retards the transformation of titanate into $\mathrm{TiO}_{2}$, thus shifting the formation of anatase phase to higher temperatures [363]. When the gold decoration procedure (reduction with hydrogen) resulted in some larger crystallites, extra reflections due to gold were also detected. In Fig. 57(B) the gold reflections are marked at $38.2^{\circ}(111), 44.4^{\circ}$ (200), $62.5^{\circ}$ (220) and $77.5^{\circ}(311)$.

$\mathrm{H}_{2} \mathrm{O}$ washed pristine nanowires feature a mixture of different crystalline titanate forms, mostly $\beta-\mathrm{TiO}_{2}$ and $\mathrm{H}_{x} \mathrm{Na}_{(2-x)} \mathrm{Ti}_{3} \mathrm{O}_{7}$ as shown in the XRD patterns (Fig. 58(A)). The $\beta-\mathrm{TiO}_{2}$ phase was identified on the basis of its reflections with Miller indices of (200), (110), (002), (111), (003), (020), (022), (711), (313), (023) and $(712)$ at $15.4^{\circ}, 24.9^{\circ}, 28.6^{\circ}, 29.4^{\circ}, 43.5^{\circ}, 48.5^{\circ}, 57.3^{\circ}$, $58.3^{\circ}, 61.7^{\circ}, 68.2^{\circ}$ and $76.8^{\circ}$, respectively. The $\mathrm{H}_{x} \mathrm{Na}_{(2-x)} \mathrm{Ti}_{3} \mathrm{O}_{7}$ phase was identified on the basis of its reflections with Miller indices of (001), (101), (011), (300), (203) and (401) found at $10.5^{\circ}, 15.8^{\circ}, 25.7^{\circ}, 29.9^{\circ}, 34.2^{\circ}$ and $43.9^{\circ}$, respectively. The nanowires preserve the wire-like morphology during the heat treatment up to $873 \mathrm{~K}$. However, more and more textural 

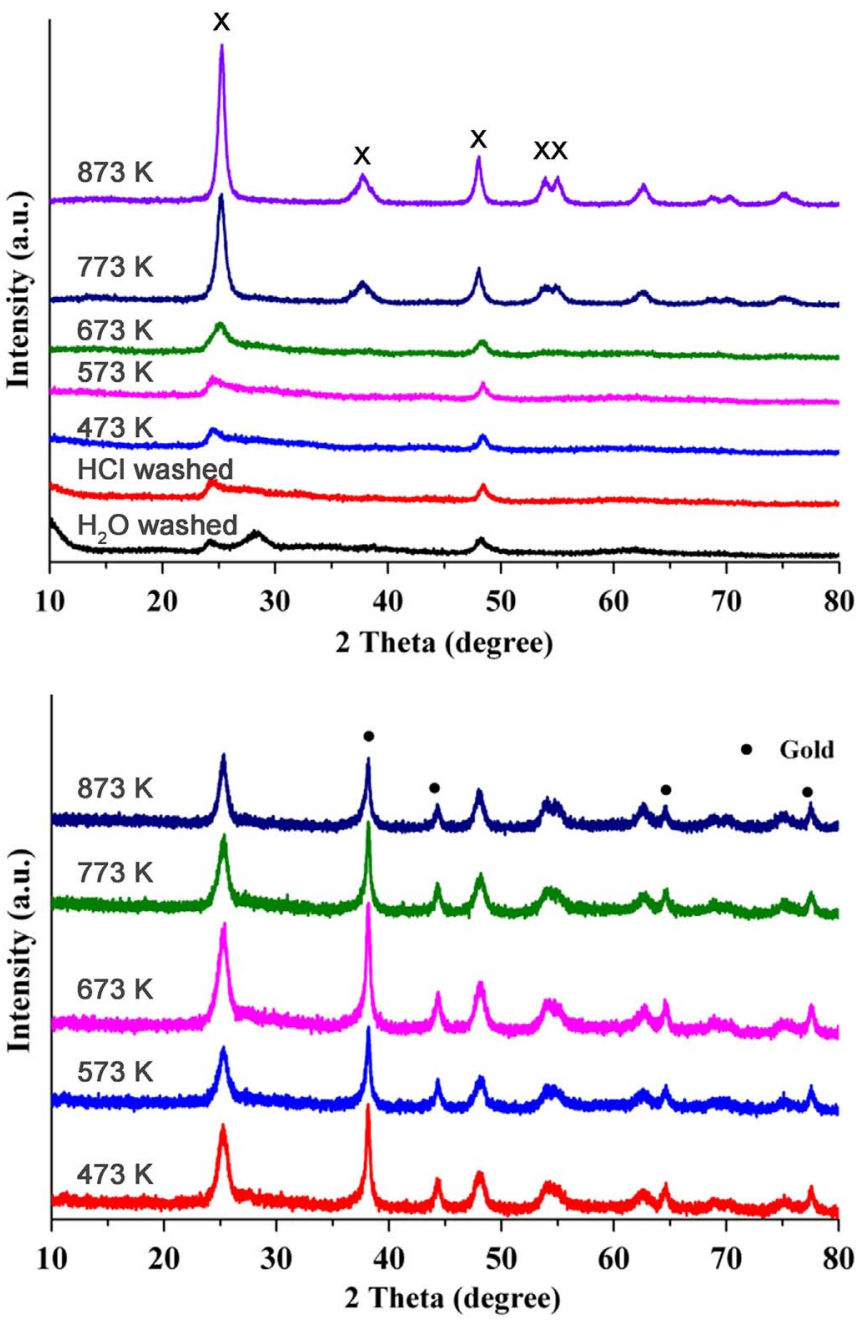

Fig. 57. XRD of $\mathrm{H}_{2} \mathrm{O}$ washed and $\mathrm{H}$-form titanate nanotubes (upper part) and $\mathrm{Au}$ loaded (2.5\%) nanotubes (lower part) as a function of annealing temperature ("X" denotes the anatase phase). Reproduced from Ref. [52].

discontinuities can be observed at higher temperatures. The holey structure can be attributed to the continuous transformation of protonated titanate nanostructures to $\mathrm{TiO}_{2}$ (anatase) followed by water formation and release from the structure. These processes result in the rearrangement of the anatase crystals and the appearance of voids in the structure of the nanowire (Fig. 58(A)).

The XRD profiles of gold decorated nanowires are shown in Fig. 58(B) as a function of heat treatment. The most important result is that unlike in titanate nanotubes and in undecorated titanate nanowires, gold inhibited the transformation of the titanate nanowires into anatase. No reflexions can be assigned to anatase in the diffractograms of the thermally annealed samples, the visible reflexions are all due to gold particles: $38.2^{\circ}(111), 44.4^{\circ}(200), 64.5^{\circ}(220)$ and $77.5^{\circ}$ (311). It is worth noting that the effect of gold adatoms on the structure and stability of titanate nanowires is significantly different from those of $\mathrm{Rh}$. It was previously demonstrated that $\mathrm{Rh}$ induces the transformation of the wire structure to $\beta-\mathrm{TiO}_{2}$ above $573 \mathrm{~K}$ [183]. Although the possible presence of a $\beta$ $\mathrm{TiO}_{2}$ like phase was also indicated in the present case by the
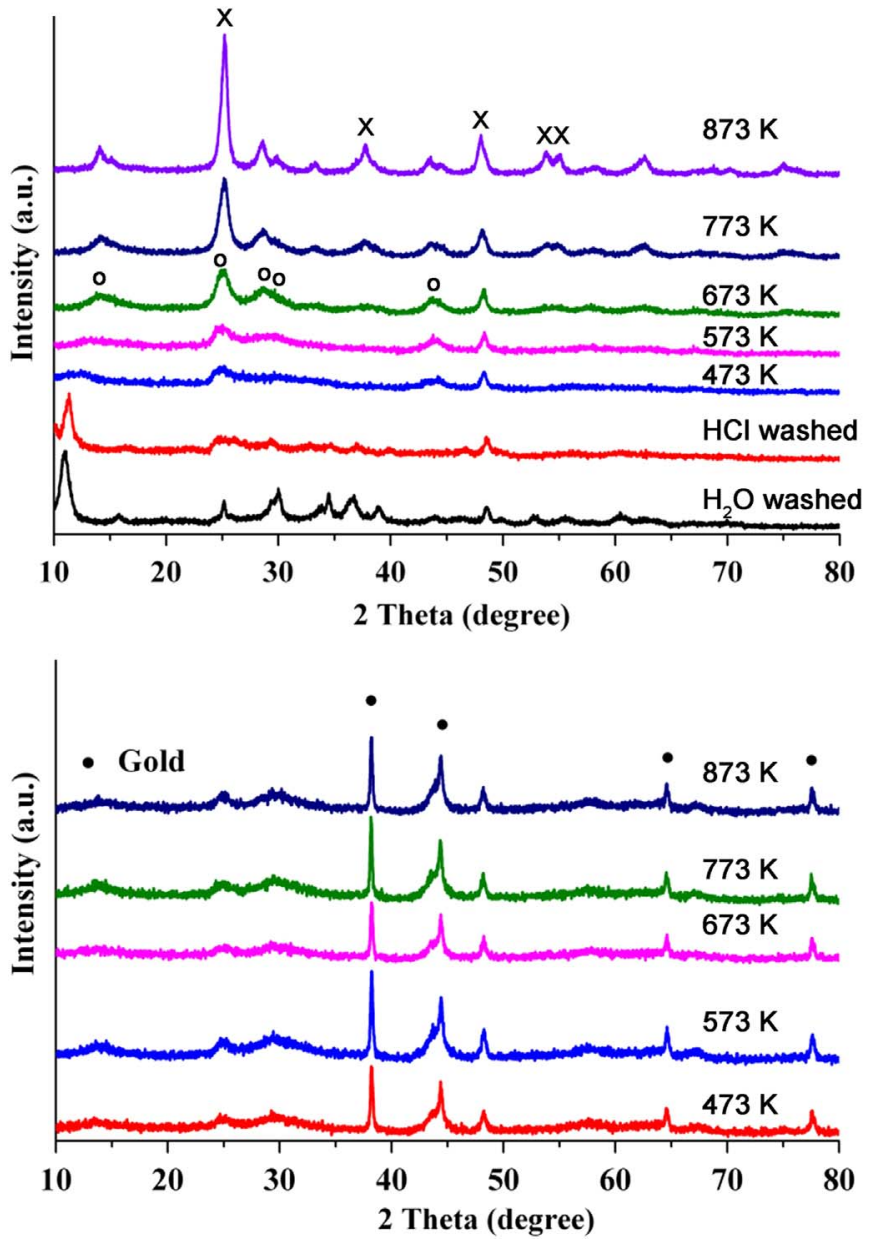

Fig. 58. XRD of $\mathrm{H}_{2} \mathrm{O}$ washed and $\mathrm{H}$-form titanate nanowires (upper part) and $\mathrm{Au}$ loaded (2.5\%) nanowires (lower part) as a function of annealing temperature (' $\mathrm{X}$ ' denotes the anatase, 'o' denotes the $\beta-\mathrm{TiO}_{2}$ phase). Reproduced from Ref. [52].

Raman spectra (Fig. 56(D)), the lack of clear evidence of $\beta$ $\mathrm{TiO}_{2}$ reflections in the XRD profiles does not allow us to unambiguously identify the thermal annealing products of gold decorated titanate nanowires as $\beta-\mathrm{TiO}_{2}$.

For this apparent contradiction a possible explanation can be offered by considering that titanate nanowires are actually rather bulky objects: their approx. $100 \mathrm{~nm}$ diameter corresponds to a composition made of several hundreds of titanate layers. Thus, it is possible that only the topmost few layers are converted into $\beta-\mathrm{TiO}_{2}$ upon heating (see below for details). This would be visible very well in the Raman spectrum, however, the "bulk phase" of the material would still be titanate and consequently, the XRD profile would be dominated by the titanate signature reflections. On the other hand, the walls of a titanate nanotube consist of only a few layers, therefore, any structural changes are simultaneously reflected by their Raman and XRD response.

TEM images demonstrate the tubular morphology of the as-synthesized titanate nanotubes with a diameter of $\sim 7 \mathrm{~nm}$ and length up to $80 \mathrm{~nm}$. The acid washing process resulted in a mild destruction of the inner and outer nanotube walls. 
In agreement with the XRD results no morphological degradation could be observed after heat treatment up to $573 \mathrm{~K}$. However, when the titanate nanotubes were decorated with gold, the tube structure was destroyed as low as at $473 \mathrm{~K}$. The HRTEM images are presented from $473 \mathrm{~K}$ in Fig. 59(B)-(D) in two resolutions; in $100 \mathrm{~nm}$ and $20 \mathrm{~nm}$, respectively. For comparison $\mathrm{H}$-form titanate nanotubes without gold are also displayed in Fig. 59(A). The morphology did not change any further up to $878 \mathrm{~K}$. These HRTEM results agree well with our Raman and XRD findings (Figs. 56 and 57) and confirm that the gold additive promotes the development of anatase phases.

HRTEM images of gold-decorated $\mathrm{H}$-form titanate nanowires subjected to annealing at different temperatures are shown in two resolutions (100 nm and $20 \mathrm{~nm})$ in Fig. 60(B)(D). A characteristic image of pristine $\mathrm{H}$-form titanate nanowires is also presented in Fig. 60(A) as reference. In agreement with the XRD results, the HRTEM images have confirmed that the nanowires preserve their wire-like morphology up to $873 \mathrm{~K}$. The important new finding is that the thermal annealing behavior of Au loaded titanate nanotubes and nanowires is different. The former lose their tubular morphology and are readily transformed into anatase even at the very low temperature of $473 \mathrm{~K}$. On the other hand, gold stabilizes the layered structure of titanate nanowires and prevents anatase formation up to $873 \mathrm{~K}$. The morphology stabilization effect of gold was independent from the method used for its reduction. HRTEM results show that the wire-like morphology is stable with the temperature even the gold nanoparticles were prepared by $\mathrm{NaBH}_{4}$ reactant. It seems that the effect of gold is different from that of rhodium [183]: while Rh-decorated nanowires were unambiguously shown to transform into $\beta-\mathrm{TiO}_{2}$ earlier, in thermally annealed Au-decorated nanowires the presence of a $\beta-\mathrm{TiO}_{2}$ phase is only suggested by the Raman spectra but not confirmed by XRD.

The main difference between titanate nanotubes and nanowires is that the specific surface area of the former is approx. one order of magnitude larger because of their hollow inner channel and thinner walls. Nanotubes are also two orders of magnitude shorter than nanowires. This implies that nanotubes offer significantly more accessible (i.e. with respect to diffusion or surface mobility) cation positions and mainly gold particles than nanowires. Indeed, previous XPS measurements revealed that heat treatment induces further gold penetration into the nanotube, whereas no similar effect is observable for nanowires [52]. Let us consider that the presence of charge compensating cations in the titanate framework unavoidably hinders the phase transition to anatase $\mathrm{TiO}_{2}$, since the cations need to physically leave the structure. Protons are very mobile and are easily removed by dehydration, this is why H-form nanotubes and nanowires exhibit the same behavior and convert into anatase upon heating. Ab initio modeling of titanate nanotubes by Szieberth et al. [370] has clearly confirmed this hypothesis.

Gold, on the other hand, is significantly less mobile than $\mathrm{H}^{+}$and more difficult to remove from the structure during thermal treatment. Nevertheless, the small size, thin walls and accessible inner channel of nanotubes make it possible for the
$\mathrm{Au}^{+}$ions to leave the titanate structure and migrate into the hollow interior where they are reduced and form small clusters. Actually, the reduction of the positively charged metal ions in the channel even helps in dehydrating the nanotube, which is a possible reason for the observed catalytic effect of gold on the conversion of trititanate nanotubes into anatase. On the other hand, gold ions compensating the negative framework charge in a several hundred layer thick titanate nanowire cannot leave the structure so easily; therefore, they prevent any phase transitions just like it was observed by XRD. Obviously, the topmost layers of the nanowire can still be depleted of gold, and thus, phase transitions in the surface layers of the nanowire may become possible. This is the most likely reason for the observation of $\beta-\mathrm{TiO}_{2}$-like Raman features without the corresponding XRD evidence for $\beta-\mathrm{TiO}_{2}$ in Au-decorated annealed nanowires [52].

In most cases in-situ XRD experiments demonstrate the transformation of transition metal doped (including Fe-doped) trititanate nanotubes to titania phase under reductive atmosphere [266]. Magnetic measurements indicate that the Fedoped trititanate nanotubes comprise a mixture of ferromagnetic and paramagnetic phases.

Comparing the experimental results accumulated by different laboratories we may summarize that $\mathrm{Na}$ inhibits, whereas $\mathrm{Au}, \mathrm{Ag}, \mathrm{Rh}, \mathrm{Fe}$ and Co catalyse the transformation of the titanate structure into a nanosized anatase phase. The titanate nanowire phase is stabilized by $\mathrm{Au}$, while $\mathrm{Rh}$ adatom induces the phase transition from wire-like structure to $\beta-\mathrm{TiO}_{2}$-like feature. All these findings are very important in materials science as well as in catalytic and photocatalytic applications.

\subsection{Non-metal and anion doped titanate nanostructures}

Now we discuss the structure of non-metal doped titanate nanowires and nanotubes. The results obtained on non-metal and anion doped $\mathrm{TiO}_{2}$ are well documented in a previous excellent review [3]. There has been an explosion in the literature on anion-doping of $\mathrm{TiO}_{2}$ since Asahi and coworkers' 2001 Science report on visible light activity in nitrogen doped $\mathrm{TiO}_{2}$ [371]. By far, the most extensively studied anion dopant has been $\mathrm{N}$, but other anion dopants e.g. C, halides, $\mathrm{P}$ and $\mathrm{B}$ have also been examinedboth experimentally and theoretically [3]. The general idea is that when substitutionally doping with a less electronegative than $\mathrm{O}$, the lattice will have some valence p-states pushed up out of the $\mathrm{TiO}_{2}$ valence band (VB) into the bandgap.

Fujishima et al. [372] have briefly described some of the preparation methods and general characteristics of anion-doped $\mathrm{TiO}_{2}$, so these will not be detailed here. It should be emphasized that doping preparation methods in general are very diverse, ranging from dry methods (e.g. ion implantation or incomplete oxidation of $\mathrm{Ti}$ nitrides, carbides, etc.) to wet methods (e.g. sol-gel synthesis).

Asahi et al. [371,373] were the first to synthetize high surface area $\mathrm{N}$-doped $\mathrm{TiO}_{2}$ (mixed anatase and rutil) and to study its properties for visible light photochemistry.These authors sputter-deposited $\mathrm{TiO}_{2}$ in $\mathrm{Ar} / \mathrm{N}_{2}$ gas mixture and 

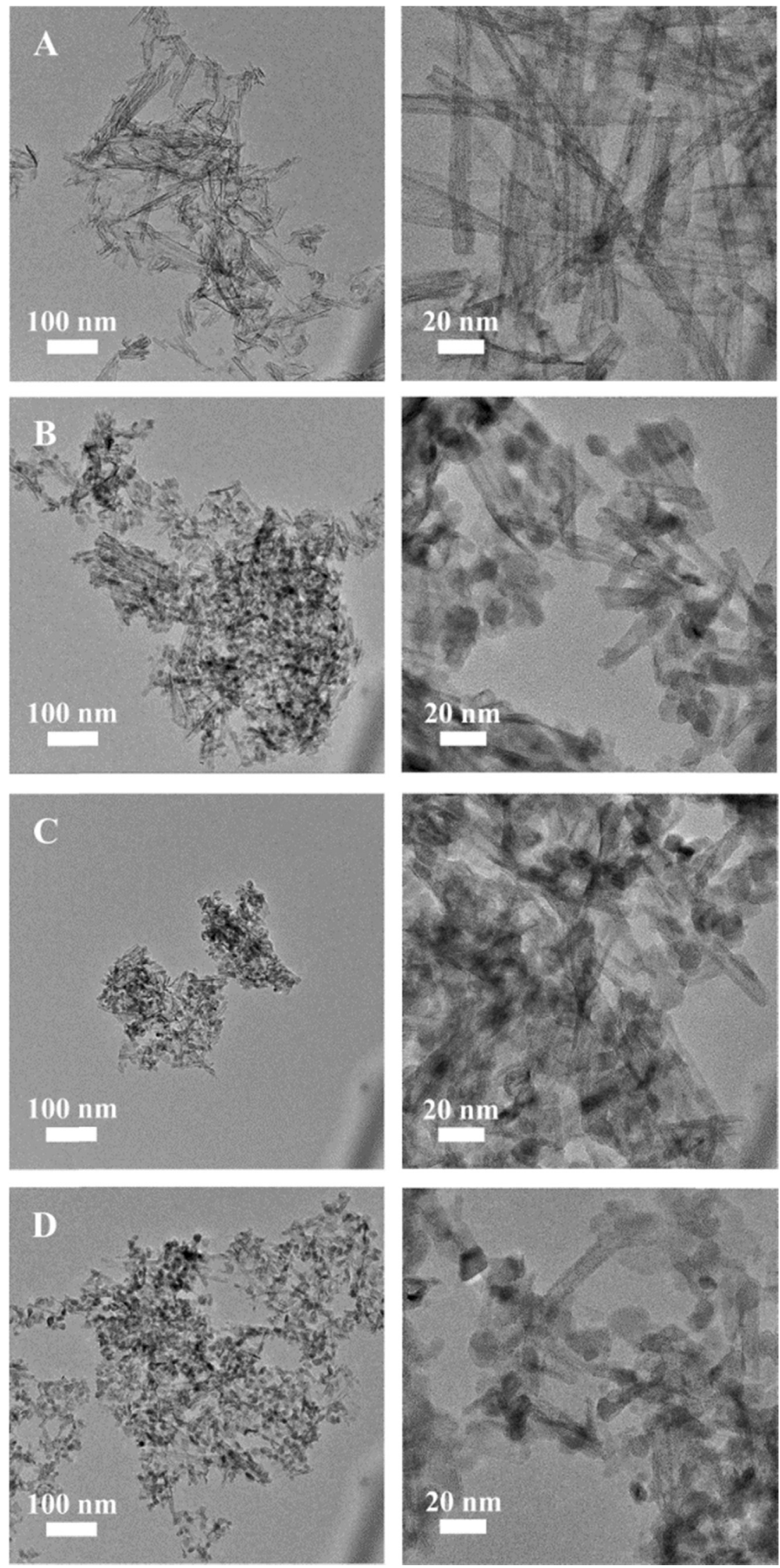

Fig. 59. HRTEM images of H-form titanate nanotubes (A) and Au containing (2.5\%) nanotubes after different heat treatments; (B) $-473 \mathrm{~K}$, (C) $-673 \mathrm{~K}$, (D) -873 K. Reproduced from Ref. [52]. 

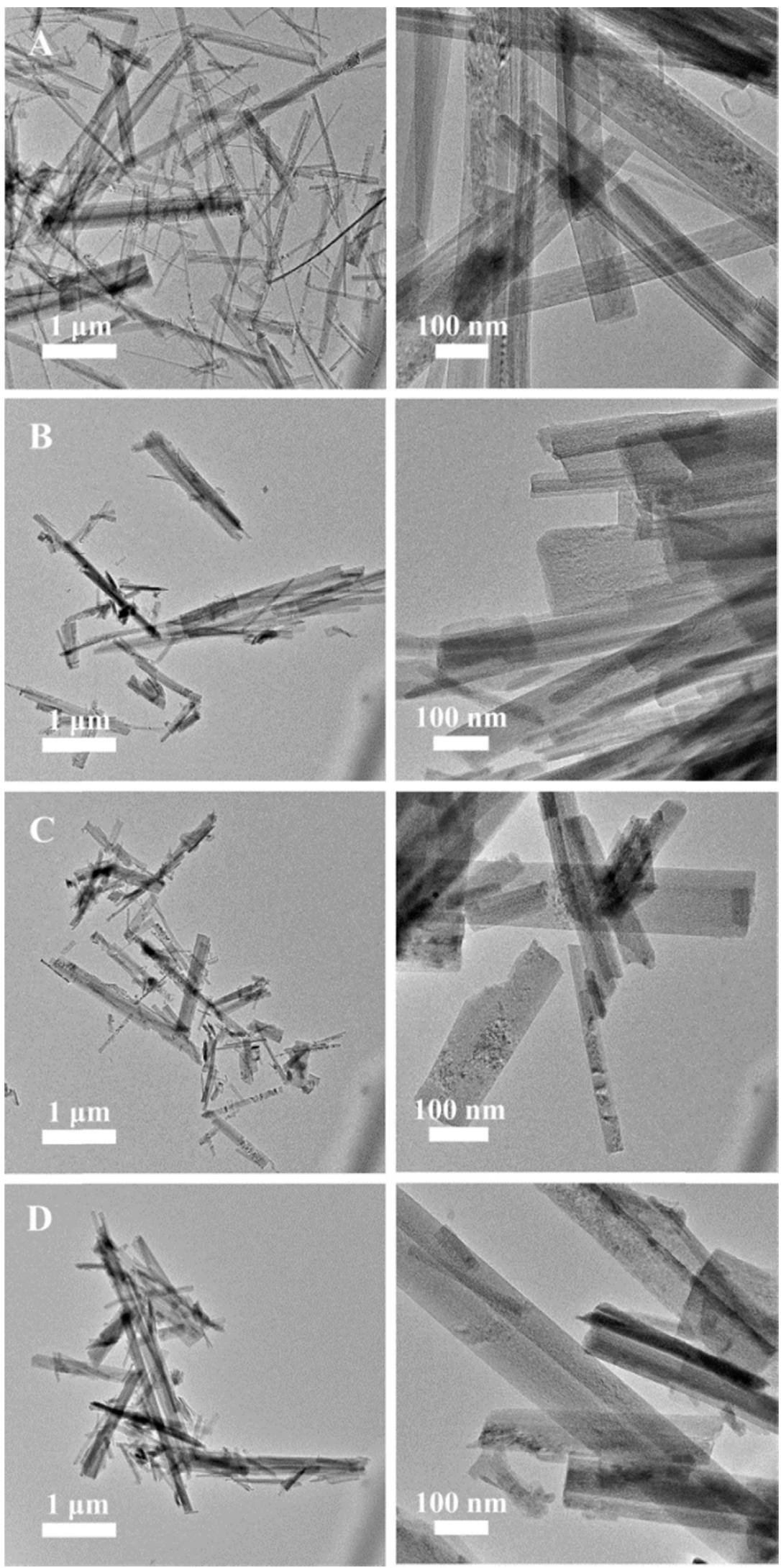

Fig. 60. HRTEM images of H-form titanate nanowires (A) and Au containing (2.5\%) nanowires after different heat treatments; (B) $-473 \mathrm{~K}$, (C) $-673 \mathrm{~K}$, (D) -873 K. Reproduced from Ref. [52]. 
observed a redshift in the optical absortion spectrum of the resulting film. Based on theory and a $396 \mathrm{eV}$ X-ray photoelectron spectroscopy (XPS) feature in the N 1s region, they assigned the $\mathrm{N}$ dopant to a substitutional site. While a variety of techniques has been employed to study the $\mathrm{N}$ dopant in high surface area $\mathrm{TiO}_{2}$ samples, at present the most commonly used diagnostic tool for characterizing the state of the dopant is XPS. In general, groups have assigned the dopant's site location based on this technique. Features at $\sim 396 \mathrm{eV}$ are attributed to substitutional $\mathrm{N}$ and the features at $\sim 400 \mathrm{eV}$ are due to interstitial nitrogen $[374,375]$.

In the field of titanate nanostructures the most frequently studied non-metal dopant was also $\mathrm{N}$. H-form titanate nanotubes were prepared by the alkaline hydrothermal method and subsequently doped with nitrogen obtained from the thermal decomposition of urea. The developed method offers the lowest temperature $(500 \mathrm{~K})$ route to $\mathrm{N}$-doped trititanatederived nanostructures to date [376]. The amount of incorporated nitrogen could be controlled by the duration of the reaction. Nitrogen in high concentration induced both structural and morphological changes even without any additional heat treatment. However, by calcining the doped samples it was possible to facilitate nitrogen-related transitions in the oxide morphology and crystalline phase, resulting in materials with higher crystallinity and a more regular shape.

The transformation from trititanate to the anatase/rutile phase was investigated by XRD measurements [376]. Fig. 61 shows the effect of nitrogen doping on the crystalline structures of untreated TiONT samples (Fig. 61(a)) and those calcined at $400{ }^{\circ} \mathrm{C}, \quad 600{ }^{\circ} \mathrm{C}$ and $900{ }^{\circ} \mathrm{C}$ (Fig. 61(b)-(d), respectively). In Fig. 61(a) the diffraction pattern of the undoped TiONTs agrees well with previous literature results $[38,377,378]$. After $8 \mathrm{~h}$ of nitrogen doping, anatase reflections (JCPDS card no. 21-1272) appear in addition to the remaining trititanate reflections that diminish with increasing synthesis time. The samples calcined at $400{ }^{\circ} \mathrm{C}$ and $600{ }^{\circ} \mathrm{C}$ (Fig. 61 (b) and (c)) exhibit only reflections characteristic of anatase even without any nitrogen doping. The narrowing of the (101) anatase reflection $\left(2 \theta=25.7^{\circ}\right)$ in Fig. $61(b)$ and (c) implies a higher degree of crystallization at elevated heat treatment temperatures. At a calcination temperature of $900{ }^{\circ} \mathrm{C}$ (Fig. 61 (d)) rutile reflections (JCPDS card no. 21-1276) appear besides those assigned to the anatase phase. The increase in the intensity of the (110) rutile reflection $\left(2 \theta=27.8^{\circ}\right)$ with increasing nitrogen doping indicates a phase transition induced by the insertion of nitrogen into the structure.

Nitrogen and boron codoped nanotubes showed a decrease in band gap energy as compared with undoped tubes [379]. The chemical state of $\mathrm{N}$ in codoped titanate nanotubes was investigated using high-resolution XPS. The $\mathrm{N}$ 1s spectrum shows a broad peak at around $396.8 \mathrm{eV}$, which is related to the formation of $\mathrm{O}-\mathrm{Ti}-\mathrm{N}$ bond. The formed $\mathrm{O}-\mathrm{Ti}-\mathrm{N}$ bond indicates that partial $\mathrm{O}$ was substituted by $\mathrm{N}$ in the lattice of $\mathrm{H}_{2} \mathrm{Ti}_{3} \mathrm{O}_{7}$.

A facile one-step cohydrothermal synthesis via urea treatment has been adopted to prepare a series of nitrogen-doped titanate nanotubes with high visible light photocatalytic activity against rhodamin B [380]. XPS was conducted for the chemical identification of the valence state of the doping nitrogen. The assignment of the XPS peak of $\mathrm{N} 1 \mathrm{~s}$ has been under dispute and some controversial hypotheses have been proposed. Some reports suggested that the N 1s peak at 399$400 \mathrm{eV}$ is due to the adsorbed $\mathrm{NH}_{3}$ on the $\mathrm{TiO}_{2}$ surface [371,381]. In most cases, the $\mathrm{N} 1 \mathrm{~s}$ peak at $\sim 400 \mathrm{eV}$ has been assigned to molecularly chemisorbed $\gamma-\mathrm{N}$ [382]. The assignment of this $\mathrm{N} 1 \mathrm{~s}$ peak is referred to an interstitial position directly bound to the lattice oxygen. These interstitial $\mathrm{N}$ atoms come from the ammonium cyanate or its ionic form $\left(\mathrm{NH}_{4}^{+}\right.$, $\mathrm{NCO}^{-}$) [383], which are the hydrolyzed products of the aqueous urea solution during the cohydrothermal synthesis process. Therefore, the $\mathrm{N}$ 1s peak can be assigned to the anionic $\mathrm{N}^{-}$in $\mathrm{Ti}-\mathrm{O}-\mathrm{N}$ or Ti-N-O linkages. Further evidence on the nitrogen linkage to both oxygen and titanium can be found from the high resolution XPS of Ti $2 p_{3 / 2}$ and $\mathrm{O} 1 \mathrm{~s}$.

$\mathrm{NH}_{4}^{+}$ions confined in the inter-layer pores in trititanate nanotube walls were utilized to prepare N-TNT from $\mathrm{NH}_{4} \mathrm{TNT}$ [384]. Calcining $\mathrm{NH}_{4} \mathrm{TNT}$ at $473 \mathrm{~K}$ decreased the inter-layer spacing of the nanotube wall and reduced the value of $E_{g}$, yielding $\mathrm{NH}_{4} \mathrm{TNT}$ that responded to visible light. Calcining $\mathrm{NH}_{4} \mathrm{TNT}$ at $573 \mathrm{~K}$ decomposed the intercalated $\mathrm{NH}_{4}^{+}$ions and formed pressurized $\mathrm{NH}_{3}$ gas within the nanotube wall that fractured and thereby shortened the nanotube. Calcination at $573 \mathrm{~K}$ also caused phase transformation from hydrogen titanate to $\mathrm{TiO}_{2}$ (B). Calcination at $673 \mathrm{~K}$ induced the dehydrogenation of $\mathrm{NH}_{3}$ that was trapped inside the nanotube wall, producing interstitial $\mathrm{NH}_{2}$ species in the nanotube wall. Therefore, calcination of $\mathrm{NH}_{4} \mathrm{TNT}$ at between 573 and $673 \mathrm{~K}$ results in the formation of $\mathrm{N}-\mathrm{TiO}_{2}$ (B) nanotubes and $\mathrm{N}$ anatase nanotubes, respectively. Calcination at $\geq 773 \mathrm{~K}$ caused the loss of $\mathrm{N}$ species from $\mathrm{N}-\mathrm{TiO}_{2}$ and the collapse of its tubular pore. The process was followed by XPS and DRIFTS techniques.

XPS spectra of $\mathrm{N}$ species in $\mathrm{NH}_{4} \mathrm{TNT}$ calcined between 383 and $873 \mathrm{~K}$ are displayed in Fig. 62. The spectrum corresponding to the N-TNT sample that was dried at $383 \mathrm{~K}$ includes only two $\mathrm{N}$ signals with $\mathrm{N}(1 \mathrm{~s})$ binding energies of $400.9 \mathrm{eV}$ and $399.6 \mathrm{eV}$. The XPS spectra did not include the typical N (1s) peak at $396 \mathrm{eV}$ from a substituted atomic $\mathrm{N}$ species in the $\mathrm{TiO}_{2}$ lattice (Ti-N, a b-N species) which has been obtained elsewhere from many $\mathrm{N}-\mathrm{TiO}_{2}$ catalysts [371]. Calcination at $573 \mathrm{~K}$ substantially reduced the intensity of the $\mathrm{N}$ (1s) peak at $400.9 \mathrm{eV}$, which vanished completely following calcination at $673 \mathrm{~K}$.

Whereas the $\mathrm{BE}$ for the $\mathrm{N}(1 \mathrm{~s})$ peak at $400.9 \mathrm{eV}$ was independent of the calcination temperature, that of the $\mathrm{N}(1 \mathrm{~s})$ peak at $399.6 \mathrm{eV}$ varied with the calcination temperature by shifting from $399.6 \mathrm{eV}$ at $383 \mathrm{~K}$ to $399.2 \mathrm{eV}$ at $773 \mathrm{~K}$ and vanishing completely at $873 \mathrm{~K}$. This change in $\mathrm{BE}$ was attributed to the chemical transformation of $\mathrm{N}$ species, aided by the shrinking of the interlayer space due to the increase in calcination temperature. Based on the XPS and the DRIFTS results, the nitrogen species associated with the $399.6 \mathrm{eV}$ peak at $383 \mathrm{~K}$ was attributed to the adsorbed $\mathrm{NH}_{3}$ molecules in the interlayer region of the nanotubes. As the calcination temperature increased, some of the $\mathrm{NH}_{3}$ molecules were desorbed and 

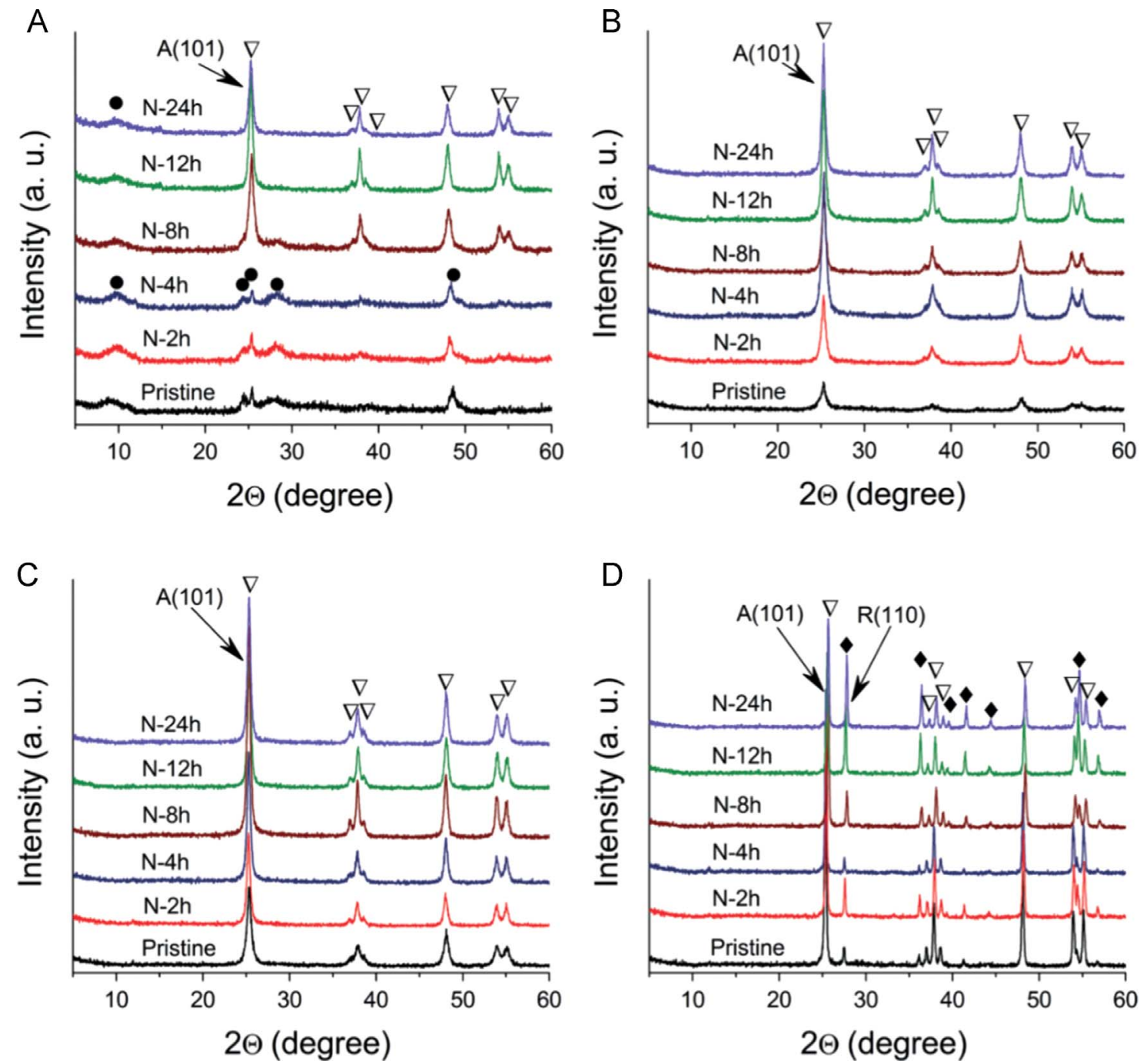

Fig. 61. Variation of the XRD patterns of TiONTs before heat treatment (a) and after calcination at $400{ }^{\circ} \mathrm{C}(\mathrm{b}), 600{ }^{\circ} \mathrm{C}$ (c), and $900{ }^{\circ} \mathrm{C}$ (d). Reflections assigned to

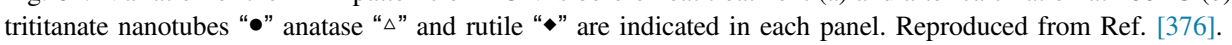

others were transformed into hydrogen-deficient nitrogen species' such as $\mathrm{NH}_{2}$. The hydrogen-deficient $\mathrm{NH}_{2}$ species' were formed by the dehydrogenation of $\mathrm{NH}_{3}$, which was accompanied by the appearance of new $\mathrm{OH}$ and $\mathrm{NH}_{2}$ vibrational peaks in the DRIFTS spectra. Yates' group recently observed an $\mathrm{N}(1 \mathrm{~s})$ peak at $399.6 \mathrm{eV}$ from an $\mathrm{N}$-doped rutile (110) surface, which was produced by annealing the single crystalline surface in $\mathrm{NH}_{3}$. The species may have been the $\mathrm{N}$ species, possibly bound to hydrogen, at an interstitial site in $\mathrm{TiO}_{2}$ [385]. Yates et al. also showed that this $\mathrm{N}$ species was responsible for narrowing the band gap of rutile (110) down to $2.4 \mathrm{eV}$ [385].

Therefore, the nitrogen species in $\mathrm{NH}_{4} \mathrm{TNT}$ that responded to visible light at 473 and $573 \mathrm{~K}$ treated nanotubes were molecular nitrogen species such as $\mathrm{NH}_{4}^{+}$and $\mathrm{NH}_{3}$, and those in $\mathrm{NH}_{4}$ TNT calcined at $\geq 673 \mathrm{~K}$ were the hydrogen-deficient species, such as $\mathrm{NH}_{2}$. Furthermore, since most of the $\mathrm{NH}^{4+}$ ions in $\mathrm{NH}_{4} \mathrm{TNT}$ were intercalated in the interlayer region of the nanotube wall, the $\mathrm{N}$ species that were generated by heating $\mathrm{NH}_{4} \mathrm{TNT}$ were interstitial $\mathrm{N}$ species.
Nitrogen and sulfur co-doped $\mathrm{TiO}_{2}$ nanosheets with exposed $\{001\}$ facets $\left(\mathrm{N}-\mathrm{S}-\mathrm{TiO}_{2}\right)$ were prepared by a simple mixingcalcination method using the hydrothermally prepared $\mathrm{TiO}_{2}$ nanosheets powder as a precursor and thiourea as a dopant [386]. The resulting samples were characterized by transmission electron microscopy, X-ray diffraction, $\mathrm{N}_{2}$ adsorptiondesorption isotherms, $\mathrm{X}$-ray photoelectron spectroscopy and UV-vis absorption spectroscopy. First principle DFT calculations confirmed that $\mathrm{N}$ and $\mathrm{S}$ co-dopants can induce the formation of new energy levels in the band gap, which is associated with the response of $\mathrm{N}-\mathrm{S}-\mathrm{TiO}_{2}$ nanosheets to visible light irradiation [386]. The enhanced activitiy of $\mathrm{N}-$ $\mathrm{S}-\mathrm{TiO}_{2}$ can be primarily attributed to the synergetic effects of two factors including the intense absorption in the visible light region and the exposure of highly reactive $\{001\}$ facets of $\mathrm{TiO}_{2}$ nanosheets.

Protonated titanate nanotubes and nanowires were doped with different amounts of boron via impregnation with $\mathrm{B}_{2} \mathrm{O}_{3}$. The B-doped structures were studied by XPS and HRTEM [387]. At room temperature the boron exists in oxidized state 


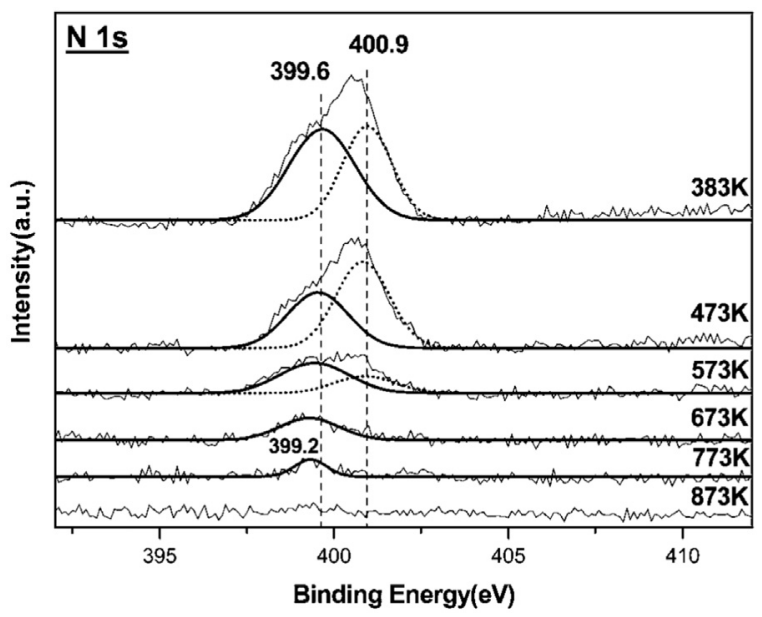

Fig. 62. XPS spectra of $\mathrm{N}$ species in $\mathrm{NH}_{4} \mathrm{TNT}$ calcined at between 383 and 873 K. Reproduced from Ref. [384].

in $\mathrm{B}_{2} \mathrm{O}_{3}$ form (Fig. 63). When the doped structues were heated to higher temperatures, significant changes were detected in the XP spectra of both nanotubes and nanowires. The intensities radically decreased and in addition the B 1s shifted to lower binding energy $(192.4 \mathrm{eV})$. The intensity change can be explained by the dimerization of $\mathrm{B}$ which was observed on different substrates: $\mathrm{B}-\mathrm{B}$ interaction was detected on $\mathrm{Rh}$ [388,389], Fe [390] and Mo [391] surfaces. The binding energy shift to $192.4 \mathrm{eV}$ may be due to the formation of certain kinds of suboxide-like species [388], which contain groups and may form linear chains containing B-B bonds. The presence of $\mathrm{Ti}-\mathrm{O}-\mathrm{B}$ structures in the linear chain cannot be exluded. The fact that after ion etching the B 1s intensity does not change indicates that the suboxide-like species do not penetrate to the subsurface significantly.

Multi-walled (B, N)-doped titanate nanotubes (TNTs) were prepared by a simple hydrothermal method. The effect of the doping amounts of $\mathrm{B}$ and $\mathrm{N}$ on the photocatalytic activity of TNTs was studied [379]. The chemical states of B and $\mathrm{N}$ in codoped TNTs were investigated using high-resolution XPS. The peak at around $191.8 \mathrm{eV}$ appears in the B 1s spectrum, which can be ascribed to Ti-O-B bond [392]. The formed Ti-O-B structures suggest that $\mathrm{B}$ could be localized at the intersticial position or act as substitute for the $\mathrm{H}$ in the lattice of $\mathrm{H}_{2} \mathrm{Ti}_{3} \mathrm{O}_{7}$.

\section{Titanate nanowires and nanotubes as supports in catalytic reactions}

Nanostructured titanates are characterized by a relatively high specific area, which is typically in the range of 180$300 \mathrm{~m}^{2} \mathrm{~g}^{-1}$ for nanotubes and $20-50 \mathrm{~m}^{2} \mathrm{~g}^{-1}$ for nanowires (nanofibers or nanorods). These values contrast with $\sim 20 \mathrm{~m}^{2}$ $\mathrm{g}^{-1}$ for $\mathrm{TiO}_{2}$ nanotubular arrays produced by electrochemical ways. The range of pore size (from 2 to $10 \mathrm{~nm}$ ) ranks these materials as mesoporous; such structures are widely used as supports in heterogeneous catalytic processes. The high surface area of the support facilitates high dispersion of the catalyst, while the open mesopores provide efficient transport of both reagents and products [32].

Protonated nanowires and nanotubes have good ion exchange properties. This allows an atomic-scale distribution of metal cations in the titanate lattice. A suitable choice of the ionic form of the metal precursor can significantly help to increase catalyst loading and to maintain high catalysts dispersion. Titanate materials, in which anionic $\mathrm{TiO}_{6}$ units are countered by other metal cations in a variety of crystal structures are important and employed for instance in catalysis. In addition, the ionic form of metal may increase the catalytic activity in those cases where the redox mechanism is important. In the previous review, a branch of catalytic processes are well-documented [32]. In this section some additional catalytic reactions are briefly discussed without completeness due to the high number of reactions studied, which could be important from the energy carrier and environmental points of view.

The most widely studied nanostructured supported catalyst is gold on titanate nanotubes and nanowires (Au/TiONT, TiONW). It was found that gold nanoparticles could be supported successfully on $\mathrm{TiO}_{2}$ nanotubes when applied using the deposition-precipitation method. The catalytic activity of an $\mathrm{Au} / \mathrm{TiO}_{2}$ nanotube for $\mathrm{CO}$ oxidation and $\mathrm{H}_{2}$ oxidation was examined and found to be different from $\mathrm{Au}$ on anatase or rutile $\mathrm{TiO}_{2}$ catalyst [323].

Fig. 64 shows the catalytic activity of the $\mathrm{Au} / \mathrm{TiO}_{2}$ nanotube for $\mathrm{CO}$ oxidation and $\mathrm{H}_{2}$ oxidation. The temperature where the conversion of $\mathrm{CO}$ and $\mathrm{H}_{2}$ reaches $50 \%\left(T_{1 / 2}\right)$ was between 310 and $330 \mathrm{~K}$ for $1 \mathrm{vol} \% \mathrm{CO}$ and $\mathrm{H}_{2}$ in air. This activity for $\mathrm{CO}$ oxidation is considerably lower compared with $\mathrm{Au}$ on $\mathrm{TiO}_{2}$ powder catalyst such as $\mathrm{P}-25$, the $T_{1 / 2}$ of which was $\sim 220 \mathrm{~K}$.

The activity for hydrogen oxidation is similar to the standard $\mathrm{Au}$ on $\mathrm{TiO}_{2}$ catalyst. It seems that the contact structure in $\mathrm{Au} /$ $\mathrm{TiO}_{2}$ nanotube is different from standard $\mathrm{Au} / \mathrm{TiO}_{2}$ catalyst because the catalytic activity for $\mathrm{CO}$ oxidation is sensitive to the contact structure with support [393]. The activity of $\mathrm{H}_{2}$ oxidation tends to depend on the surface area of $\mathrm{Au}$ particles. The crystalline structure of the $\mathrm{TiO}_{2}$ support affects the contact structure, such as crystalline orientation relationship [394]. Surface defect of the support affects the strength of contact between $\mathrm{Au}$ and $\mathrm{TiO}_{2}$. The catalytic properties also indicate that the contact structure between $\mathrm{Au}$ and $\mathrm{TiO}_{2}$ nanotube is different from that of $\mathrm{Au}$ on anatase or rutile $\mathrm{TiO}_{2}$. This indicated that the Au catalyst is sensitive to the structure of the support and the interface between Au and support [394].

Titanium oxide nanotubes were synthesized and used as support for $\mathrm{Au}$ nanoparticles obtained by deposition-precipitation with urea. The activity of catalysts prepared under different conditions was tested in the oxidation of $\mathrm{CO}$. The results indicate that titania nanotubes (TN) can be used as support for gold nanoparticles obtained by deposition-precipitation, since adequate gold nanoparticles $(<5 \mathrm{~nm}$ ) for $\mathrm{CO}$ oxidation can be obtained. Increasing gold content above $3 \mathrm{wt} \%$ does not improve the activity because, at $9 \mathrm{wt} \%$ Au the particle sintering increases leading to particle diameter larger than $5 \mathrm{~nm}$, which are not effective for CO oxidation [395]. 
nanotube

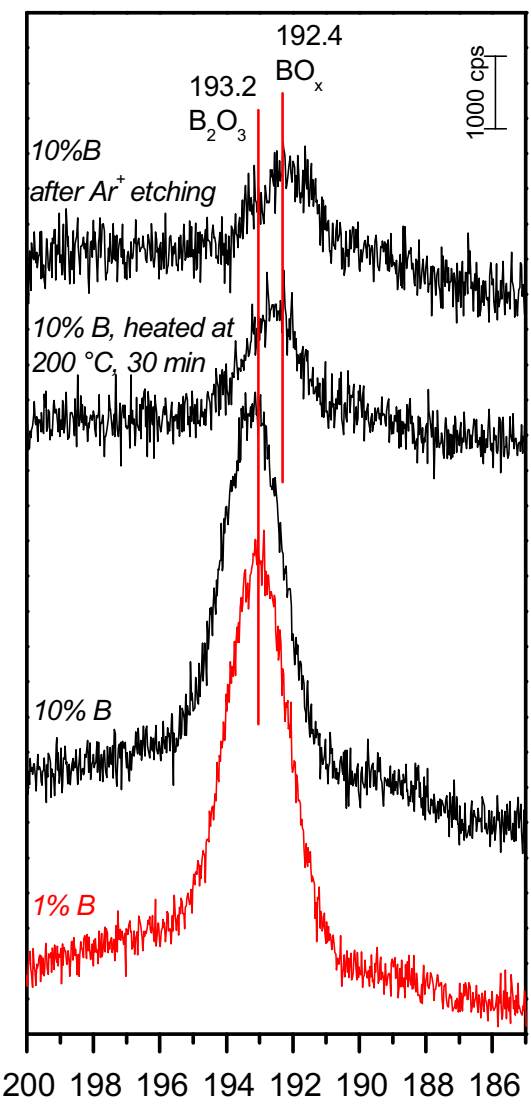

nanowire

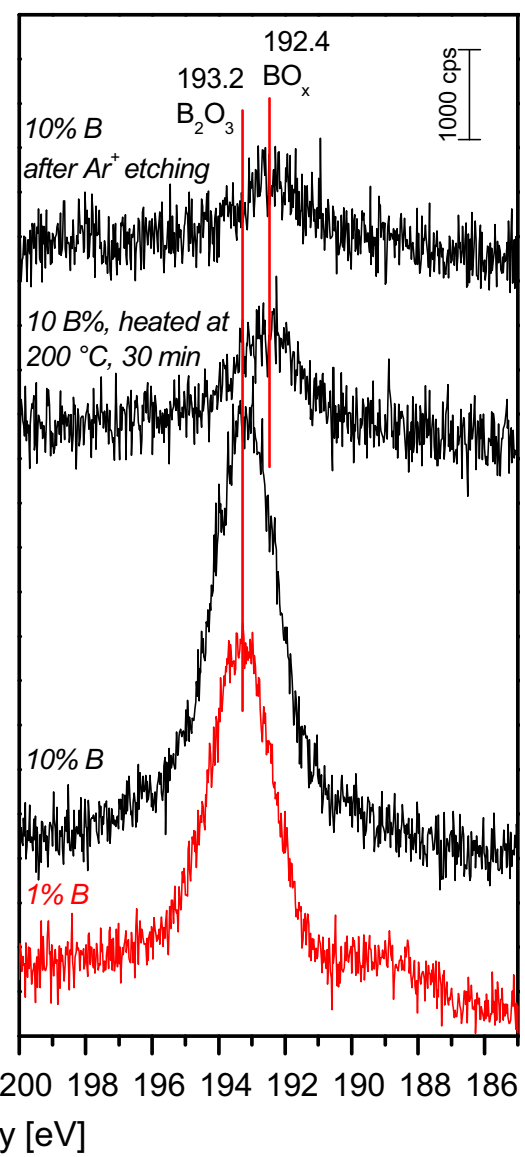

Fig. 63. B 1s Xp spectra on titanate nanotubes and nanowires during heat treatment and after ion eching.

Titanium oxide nanotubes with diameter of $8-10 \mathrm{~nm}$ and lengths of several tens to several hundred of nanometers have been used as support for preparation of nano-sized gold based catalyst [396].The catalyst was active in the water-gas shift reaction. It is quite interesting to observe that nano-sized gold can well fit into nanotubes. The application potential of the prepared $\mathrm{Au} / \mathrm{TNTs}$ was investigated in water-gas shift reaction. The observed catalytic behavior of gold-supported TNTs correlated with the structural particularity and the nature of the TNTs. The catalytic performance of Au/TNTs may be improvable by increasing the diameter of the nanotubes or modifying the structure of the nanotubes to anatase phase.

Fe-doped trititanate nanotubes which were prepared via wetchemistry exhibited noticeable catalytic activity in the watergas shift reaction [266]. The Fe-doped trititanate nanotubes were clearly active around $650 \mathrm{~K}$, and their catalytic activity increased as the temperature rose to 700 and $800 \mathrm{~K}$. It is wellknown that although pure $\mathrm{TiO}_{2}$ is thermodynamically able to act as a catalyst, it is inactive for kinetic reasons. The control experiments show the inactivity of pure $\mathrm{H}$-form titanate nanotubes or pure $\mathrm{TiO}_{2}$ nanorods.

Very recently a comparative study on the reaction of $\mathrm{CO}+\mathrm{H}_{2} \mathrm{O}$ reaction catalyzed by gold supported on titanate nanowires and nanotubes and by rhodium supported titanate nanowires and nanotubes was carried out, respectively [397].

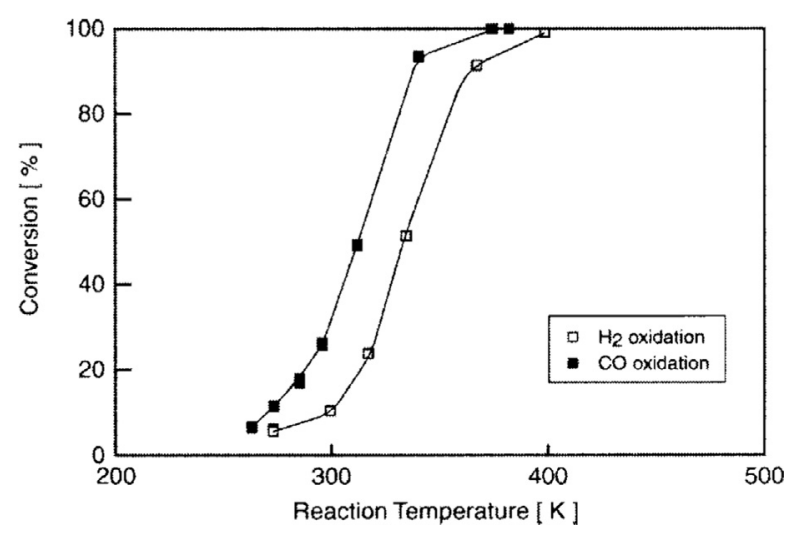

Fig. 64. Catalytic activity of $\mathrm{Au} / \mathrm{TiO}_{2}$ nanotube for $\mathrm{CO}$ and $\mathrm{H}_{2}$ oxidation. Reproduced from Ref. [323].

In the interpretation of the catalytic activities of the metals supported on titanate nanowires and nanotubes the role of the size and the oxidation states of active components were considered. The primary products in the $\mathrm{CO}+\mathrm{H}_{2} \mathrm{O}$ reaction are hydrogen and $\mathrm{CO}_{2}$ besides a trace amount of $\mathrm{CH}_{3} \mathrm{OH}$ on both $\mathrm{Au}$ and $\mathrm{Rh}$ containing titanate nanowires and nanotubes catalysts. The conversions as a function of reaction time are shown in Fig. 65. 
The Au/TiONW obtained with $\mathrm{NaBH}_{4}$ reduction has higher activity than that obtained with $\mathrm{H}_{2}$ reduction at $473-573 \mathrm{~K}$. Reduction with molecular hydrogen causes bigger particle sizes (5-9 nm) while $\mathrm{NaBH}_{4}$ reduction resulted in smaller sizes $(2-8 \mathrm{~nm})$. At the same time a higher portion of gold adatoms exists in partially positive states $\left(\mathrm{Au}^{+}\right)$after reduction with molecular $\mathrm{H}_{2}$ [52]. Finally it was concluded that the smaller Au particles play significantly more important role in the WGS reaction than the partially positive gold sizes.

A catalyst prepared from gold and deposited on titanate nanotubes has demonstrated a high activity for $\mathrm{CO}_{2}$ reduction by hydrogen [398]. Recently the investigation of this reaction was expanded to $\mathrm{Au}$ - and $\mathrm{Rh}$-doped titania and titante catalysts. Supported gold, rhodium and bimetallic rhodiumcore-gold-shell catalysts were prepared. The supports were $\mathrm{TiO}_{2}$ as well as titanate nanotube and nanowire formed in the hydrothermal conversion of titania. The catalytic properties were tested in the $\mathrm{CO}_{2}$ hydrogenation at $493 \mathrm{~K}$ [304]. It was found that $\mathrm{Rh}$ containing catalysts exhibited the highest activity among different supported catalysts. The main product was $\mathrm{CH}_{4}$ in all cases and $\mathrm{CO}$ was formed only on catalysts supported on nanotube. The methane formation rates obtained on all catalysts are displayed in Fig. 66 for comparison.

DRIFT spectra revealed the existence of $\mathrm{CO}$ and formate groups on the $\mathrm{Rh} / \mathrm{NW}, \mathrm{Rh} / \mathrm{NT}$ and on all bimetallic catalysts. Additionally, a new band around $1770 \mathrm{~cm}^{-1}$ was identified which was attributed to tilted $\mathrm{CO}$ that is bonded to $\mathrm{Rh}$ and interacts with a nearby oxygen vacancy of the support. This intermediate could dissociate easier and forms methane. Although the activity of $\mathrm{Rh}$ showed higher activity than $\mathrm{Au}$ supported catalysts it is remarkable that $\mathrm{Au}$ supported on titanate nanowires exhibited higher activity than the $\mathrm{Au} / \mathrm{TiO}_{2}$ where the support was P25 titania.

Protonated titanate nanotube (PTNT) was proved to be an effective solid acid catalyst for the hydroxyalkylation/alkylation (HAA) of 2-methyfuran (2-MF) and n-butanal from lignocellulose [399]. Compared with some often used inorganic solid acid catalysts, the PTNT has higher activity for the HAA of 2-MF with n-butanol. The summarized results are presented in Fig. 67.

According to the characterization results, the transformation of $\mathrm{TiO}_{2} \mathrm{P} 25$ to PTNT by hydrothermal treatment with $\mathrm{NaOH}$ solution and ion-exchange with acid solution leads to (1) the evident increase in the specific BET surface area and the acidity (including the amount acid sites and acid strength) of the catalyst, (2) the generation of Brönsted acid sites. All of these changes are responsible for the excellent performance of titanate nanotubes.

Nanotubular $\mathrm{TiO}_{2}$ based catalyst were synthesized for the enantioselective hydrogenation of 1-phenyl-1,2-propanedione (PPD) [400]. Cinchonidine was tethered directly with prior silanisation modification over activated TNTs. The characterization data provide evidence of the covalent immobilization of cinchonidine. The HRTEM images and XPS demonstrated that the cinchonidine content affects the active phase dispersion and particle size distribution. It can be deduced from the XPS results that the Pt nanoparticles exhibited a positively charged

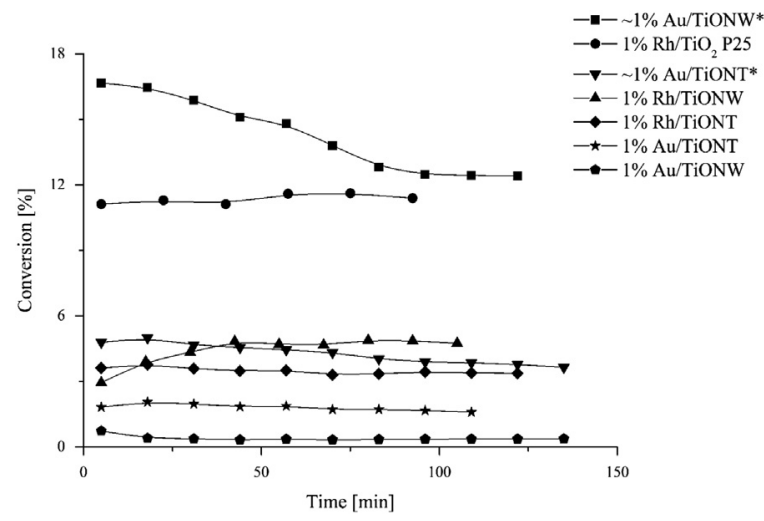

Fig. 65. Conversion data in the $\mathrm{CO}+\mathrm{H}_{2} \mathrm{O}$ reaction on $\mathrm{Au}$ and $\mathrm{Rh}$ containing titanate nanowire and nanotubes. *The samples were obtained with $\mathrm{NaBH}_{4}$ reduction at $273 \mathrm{~K}$. For comparison the activity of $\mathrm{Rh}$ supported on commercial $\mathrm{TiO}_{2}$ P25 support is also shown [397].

surface, which was attributed to metal deposition method and the strong interaction with the TNTs surface. The most selective catalyst showed poor reusability under optimum conditions and was deactivated by hydrogenation of 1trimethoxysilyl-cinchonidine (TMS-CD) and Pt leaching after the fifth cycle.

A simple two-step procedure was developed for preparing highly efficient AuNPs/TNWs composite catalyst for 4nitrophenol reduction [401]. The results showed that AuNP were crystalline with active planes such as (111), (200), (220), (311) and (222). Gold nanoparticles with an average diameter of $3 \mathrm{~nm}$ were also homogenously growing on the nanowire (TNWs) scaffold surface. Moreover, the catalytic activity of as-prepared AuNPs/TNWs nanocomposite exhibited a good catalytic activity for reduction of 4-nitrophenol. Meanwhile, it was noted that both gold particles size and gold content played an important role for influencing the catalytic efficiency of AuNPs/TNWs catalyst. This new route provided a useful platform for the fabrication of nanocatalysts based on noble metal NPs/TNWs nanocomposites.

Sodium titanate nanotubes doped with potassium were synthesized by the Kasuga method and tested as catalysts for biodiesel production [402]. Potassium was added to the nanotubes in order to increase their basicity and, consequently, improve their performance in the transesterification of soybean oil with methanol. The synthesis temperature and $\mathrm{NaOH}: \mathrm{KOH}$ molar ratio used in the preparation were changed in order to define the best experimental conditions leading to solids with nanotubular morphology and improved potassium loading. Sodium titanate nanotubes doped with potassium showed higher amounts of medium and strong basic sites than the pure sodium counterpart used as a reference. Their catalytic activity in the transesterication was also higher than that of the reference NaTNT samples. The best results were obtained with the sample containing 3.2-3.3 wt\% of potassium.

The selected examples revealed that sodium titanate nanotubes and acid treated titanates could exhibit significant catalytic activity in certain reactions by the generation of Brönsted acid sites. Metal (especially $\mathrm{Au}$ ) loaded titanates are sometimes more active catalytically than $\mathrm{Au}$ on anatase or rutile $\mathrm{TiO}_{2}$. Titanates 


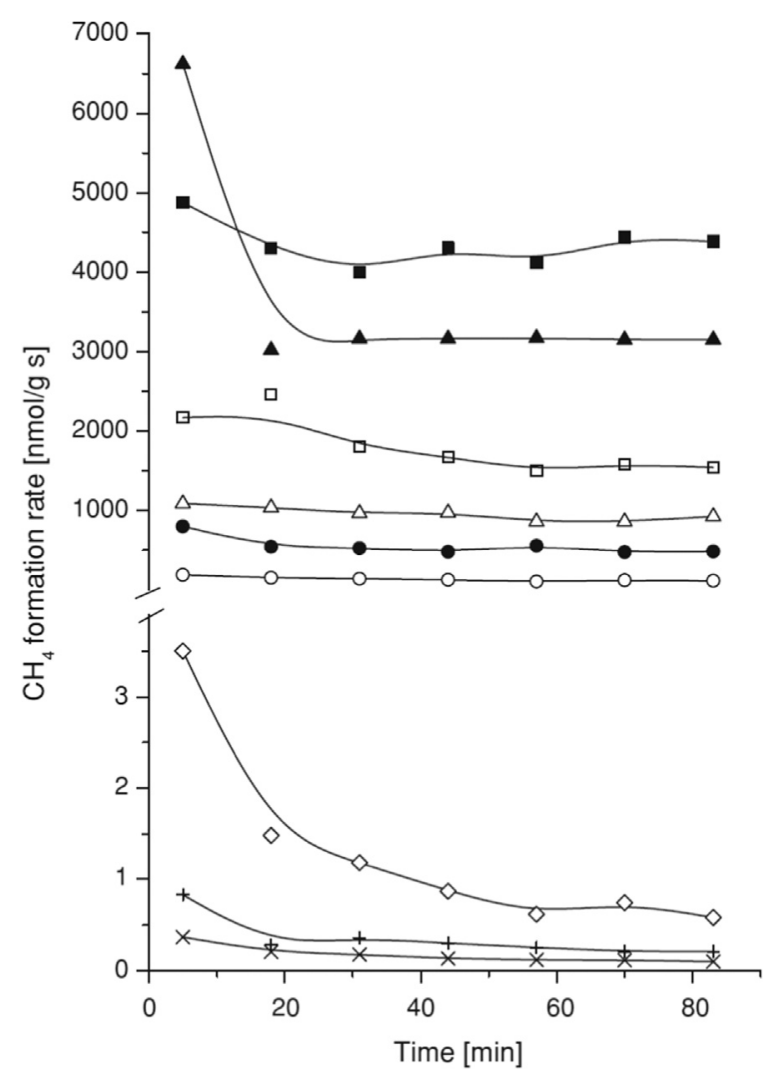

Fig. 66. Rate of methane formation on $\mathrm{Rh} / \mathrm{TiO}_{2}$ (filled square), $\mathrm{Rh} / \mathrm{NW}$ (filled triangle), $\mathrm{Rh} / \mathrm{NT}$ (filled circle), $\mathrm{Au}-\mathrm{Rh} / \mathrm{TiO}_{2}$ (open square), $\mathrm{Au}-\mathrm{RhNW}$ (open triangle), $\mathrm{Au}-\mathrm{Rh} / \mathrm{NT}$ (open circle), $\mathrm{Au} / \mathrm{TiO}_{2}$ (times symbol), $\mathrm{Au} / \mathrm{NW}$ (open diamond), AuNT (plus sign) catalysts at 493 K. Reproduced from Ref. [304].

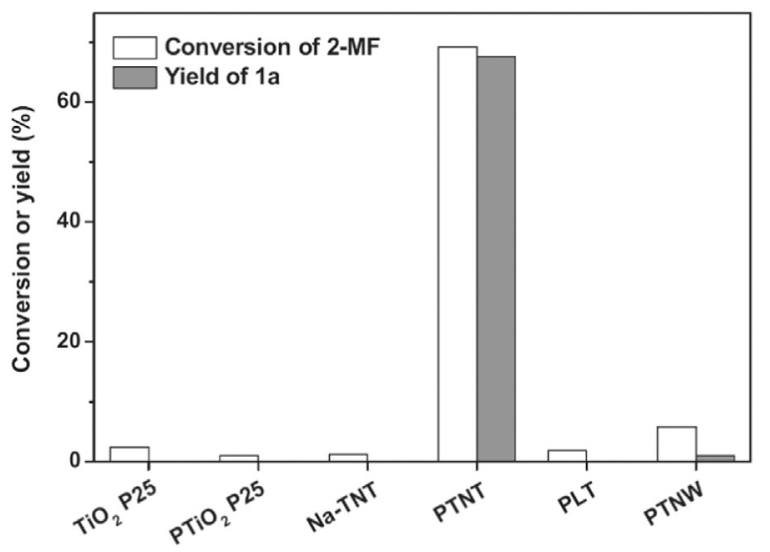

Fig. 67. Conversion of 2-MF (white bar) and the yield of 1 a (gray bar) over different titania catalysts [399].

can stabilize the gold in small sizes. Moreover, the contact structure between $\mathrm{Au}$ and nanotubes and nanowires is different from that of $\mathrm{Au}$ on anatase or rutile $\mathrm{TiO}_{2}$.

\section{Applications}

The family of titanium oxides is among the most heavily investigated oxide materials in addressing energy and environmental issues. Due to its favorable physico-chemical properties, it has been widely applied in solar cells, photocatalysts, lithium ion battery electrodes, smart coatings, etc. We refer to the many outstanding reviews already published on such applications for details, and give a brief overview of the most important results below $[3,5,29,46,184,403-407,426,438-440,486]$.

\subsection{Conversion of $1 D$ titanates into $\mathrm{TiO}_{2}$}

Depending on the synthesis method and conditions, a large number of one-dimensional titanate phases may be achieved [33] including $\mathrm{M}_{x} \mathrm{H}_{2-x} \mathrm{Ti}_{y} \mathrm{O}_{y+1}$ (where $\mathrm{M}$ is an alkali, and $\mathrm{y}$ is 2 [408,409], 3 [410], 4 [411], 6 [412], or 8 [413]), lepidocrocites $\mathrm{H}_{x} \mathrm{Ti}_{2-x / 4} \square_{x / 4} \mathrm{O}_{4} \times \mathrm{H}_{2} \mathrm{O}(x \sim 0.7, \square$ is vacancy) [414,415], spinels such as $\mathrm{Li}_{2} \mathrm{M}^{\mathrm{II}} \mathrm{Ti}_{3} \mathrm{O}_{8}$ ( $\mathrm{M}^{\mathrm{II}}$ denotes $\mathrm{Co}, \mathrm{Zn}$ or $\mathrm{Mg}$ ) [416], $\mathrm{Li}_{4} \mathrm{Ti}_{5} \mathrm{O}_{12}$ [417,418] and $\mathrm{Li}_{2} \mathrm{Na}_{2} \mathrm{Ti}_{6} \mathrm{O}_{14}$ [419], as well as the $\mathrm{TiO}_{2}(\mathrm{~B})$ phase [420]. These 1D titanates may be ionexchanged under mild acidic conditions, and the obtained $\mathrm{H}$ titanates can serve as starting materials for synthesizing various titania phases by simple annealing processes. Upon calcination in air or in inert atmosphere, titanates undergo dehydration and gradual recrystallization into titania nanostructures of similar shape $[35,421]$. The crystal structure of the forming 1D product is a function of several factors such as the temperature and duration of annealing as well as the concentration of alkaline cations in the original lattice. Practically any titania phases may be synthesized including anatase, rutile, $\mathrm{TiO}_{2}-\mathrm{B}$, and their mixtures and core-shell structures have been reported as well [422-424].

\subsection{Photocatalysis}

In photocatalysis, the band gap of the absorbers plays a pivotal role since only photons having energy higher than the gap can excite electrons from the valence band to the conduction band. Without such an event, electron and hole pairs are not generated thus photocatalytic reactions, in a classical sense, cannot take place. Accordingly, semiconductors with large band gap absorb photons of short wavelength only. In the case of titania, the height of the gap is $\sim 3.2 \mathrm{eV}$. While this is lower than that of other titanates $(\sim 3.4 \mathrm{eV}$ for $\mathrm{K}_{2} \mathrm{Ti}_{6} \mathrm{O}_{13}$ nanotubes [48,425], $\sim 3.8 \mathrm{eV}$ for $2 \mathrm{D}$ flakes of exfoliated $\mathrm{H}_{0.7} \mathrm{Ti}_{1.825} \square_{0.175} \mathrm{O}_{4} \times \mathrm{H}_{2} \mathrm{O}$ nanotubes [46]) it still necessitates UV photons with wavelengths of $\sim 390 \mathrm{~nm}$ or shorter for absorption to occur. In applications such as contaminant removal and disinfection, this may be fine since UV lamps of various geometries, light intensities and spectral windows are commercially available and may be easily installed in reactor setups. On the other hand, when the aim is at the utilization of solar radiation for e.g. renewable energy production or remediation of pollutants in an environmentally friendly manner, ordinary titania photocatalyst is far from being the optimal selection as on the surface of the Earth only $\sim 3 \%$ of the solar spectrum is in the suitable UV range. Accordingly, other semiconductors or modified $\mathrm{TiO}_{2}$ with smaller band gap are favored so that not only the UV but also visible photons may be involved in the photocatalytic reaction schemes $[8,33,426]$.

Modifying $\mathrm{TiO}_{2}$ to tune its electronic band structure and thus its optical properties has been researched for over 20 
years. Asahi and co-workers showed [371] that anionic doping of $\mathrm{TiO}_{2}$ both in interstitial and substitutional sites of the crystal can lead to a substantial change of the density of electronic states (DOS) near the Fermi level. According to the results of first-principles calculations, when doping with N, $\mathrm{C}, \mathrm{P}$ or $\mathrm{S}$, new energy levels corresponding to the mixed $\mathrm{p}$ states of the impurities and $O 2 p$ states appear in the gap. The effect is particularly strong for substitutional $\mathrm{N}$ in the $\mathrm{O}$ sites giving rise to a significant red shift of the absorption edge and visible-light induced photocatalytic activity of the material (Fig. 68).

Nitrogen doping of 1D titania nanostructures has been reported lately [427]. A number of different methods were applied to explore the effect of doping conditions on the chemistry and photocatalytic activity of the synthesized nanomaterials in various photocatalytic processes $[428,429]$. Although $\mathrm{N}$-doping occurs in $\mathrm{N}_{2}$ atmosphere, the impurity atoms are only in interstitial sites. In contrast, doping with $\mathrm{NH}_{3}$ also results in substitutional impurities.

The stability of nitrogen impurities was found to be limited at high temperatures [428]. For instance, when decorating the nanowires with metals by the means of impregnation followed by calcination, the amount of substitutional $\mathrm{N}$ decreased but interestingly the photocatalytic activity of the material in $\mathrm{H}_{2}$ evolution reaction (from ethanol water mixture) remained fairly the same.

Here, we should make also a note on evaluating the activity of photocatalytic materials. Very often, we find in the literature that a new material is claimed to have a better performance than a reference. This can be true for a selected reaction; however one shall be careful to conclude that the new material would be in general more active than the reference. In a study by Sarkar and co-workers [429], the activity of pristine, various N-doped and hydrogen reduced $\mathrm{TiO}_{2}$ nanoparticles were compared in three merely different photocatalytic processes such as (i) degradation of methyl orange under UV-B radiation, (ii) killing staphylococcus aureus bacteria with blue light and (iii) radical formation with visible light. According to the results, one shall not generalize the superiority of any catalyst, since they usually act differently in different reactions and/or conditions.

In analogy to anionic doping, also the effect of cationic impurities in the lattice on the electronic properties has been studied (Fig. 69) [430,431]. First-principle calculations showed the appearance of new energy levels in the band gap, which is in agreement with the experimentally observed decrease of apparent band gap and coloration of $\mathrm{TiO}_{2}[432,433]$. Based on the enhanced absorption in the visible spectrum thus one may expect that transition metal doped $\mathrm{TiO}_{2}$ materials in general have better photocatalytic activity than the corresponding pristine $\mathrm{TiO}_{2}$. Interestingly, practice shows that some cations improve while others decrease the photocatalytic efficiency. As found by Karakitsou and Verykios, doping with cations having valence higher than that of $\mathrm{Ti}^{4+}$ (such as $\mathrm{W}^{6+}, \mathrm{Ta}^{5+}, \mathrm{Nb}^{5+}$ ) enhance, whereas those with lower valence $\left(\mathrm{In}^{3+}, \mathrm{Zn}^{2+}, \mathrm{Li}^{+}\right)$ result in a decrease of the photocatalytic activity of $\mathrm{TiO}_{2}$ [434]. The results may be explained by the competing processes of photogeneration and recombination of electron and hole pairs. Namely, cationic impurities act as traps as well as recombination centers for the generated carriers, thus despite the enhanced optical absorption and more efficient electronic excitation the overall photocatalytic efficiency may be lower than that of pristine $\mathrm{TiO}_{2}$ [435].

Similar to other forms of titanates and titanias, several photocatalytic reactions over 1D titanates and titania structures and their doped derivatives have been demonstrated $[35,454]$. The measured activity of the materials is often far from being practical. The reason is complex. Recombination and thus short lifetime of holes and electrons as well as the lack of good catalytic sites where reactants can bind to and products can leave from are causing the limitations. In order to improve the photocatalytic efficiency, combining the $\mathrm{TiO}_{2}$ photocatalyst with other semiconducting materials (metal oxides, sulfides, selenides and nitrides) or with metals has been found to offer an excellent

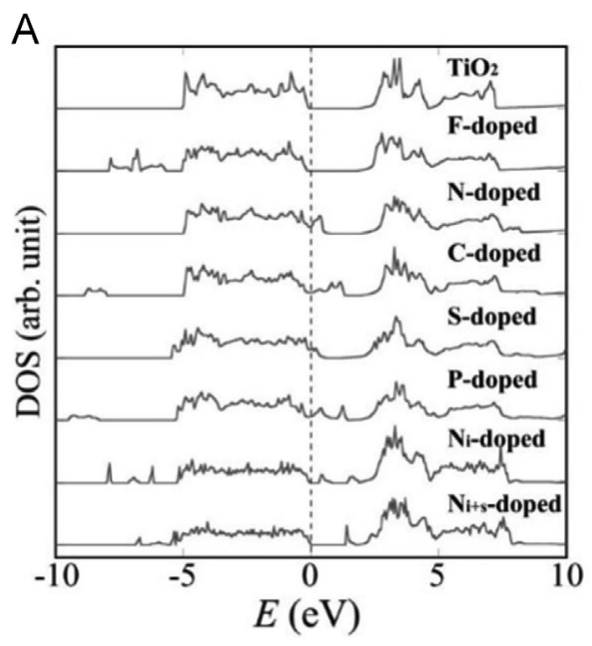

B

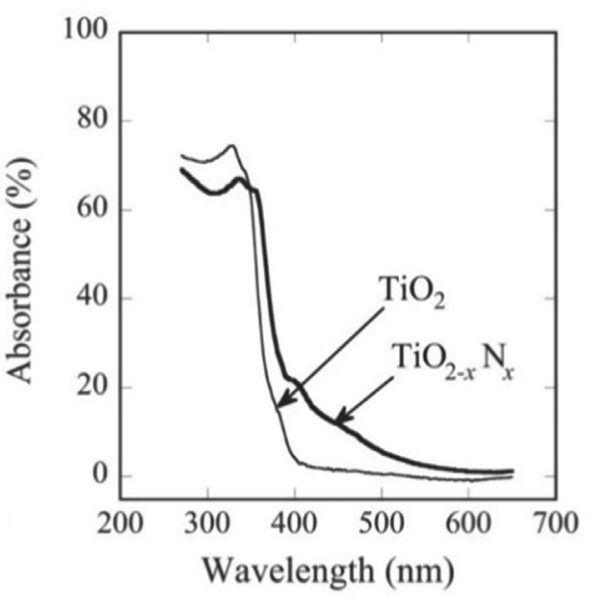

C

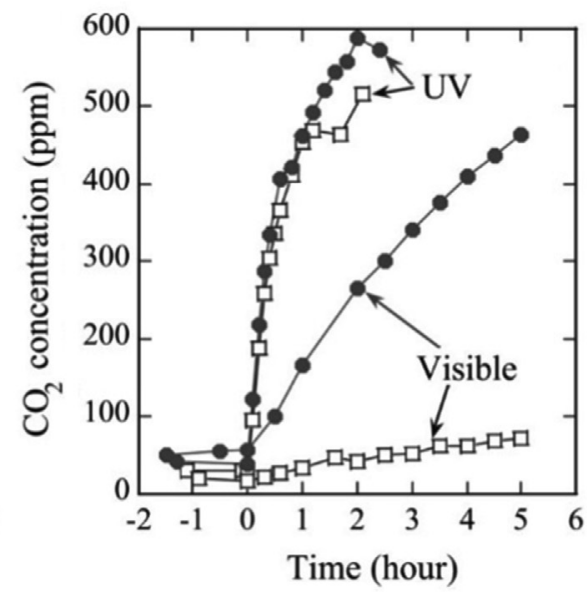

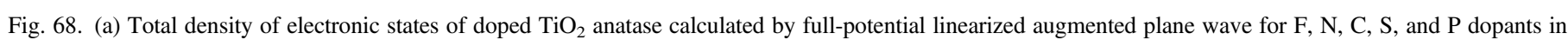

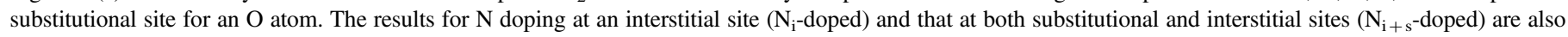

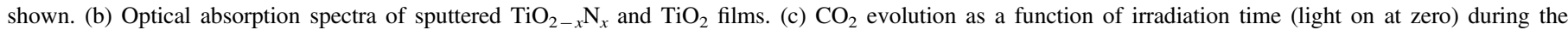
photodegradation of acetaldehyde gas under UV and visible irradiation on $\mathrm{TiO}_{2}{ }_{x} \mathrm{~N}_{x}$ (solid circles) and TiO 2 (open squares). Reproduced from Ref. [371]. 


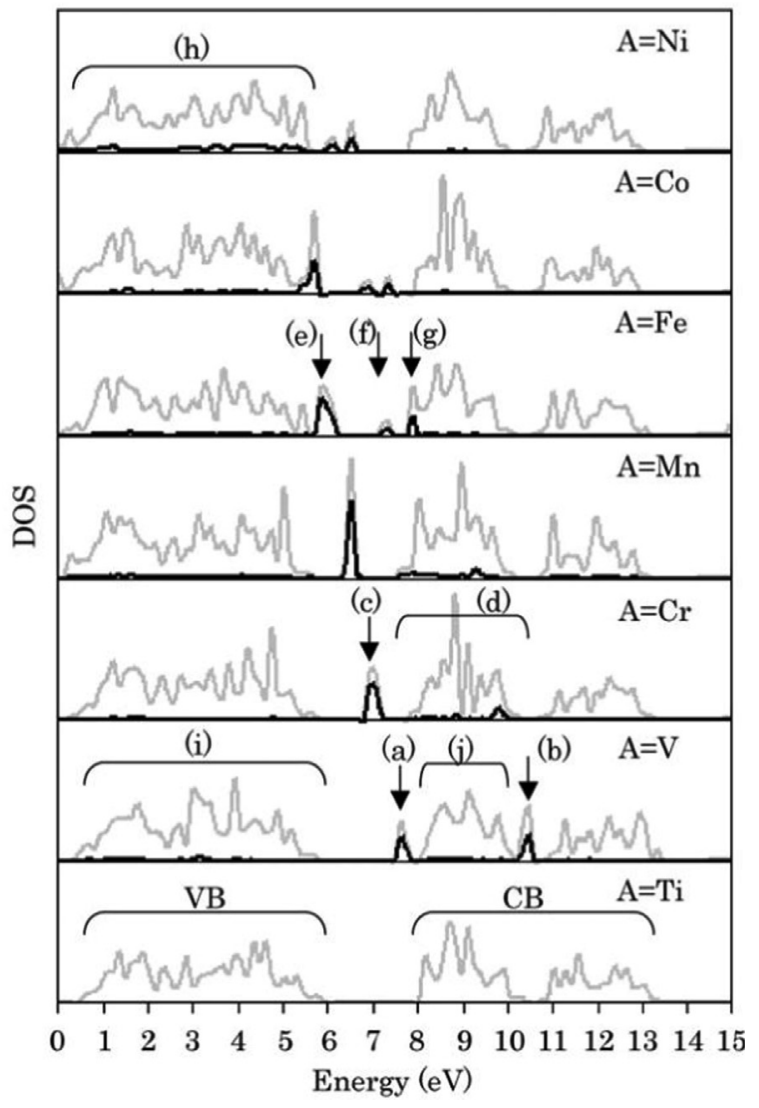

Fig. 69. The density of electronic states of metal-doped $\mathrm{TiO}_{2}\left(\mathrm{Ti}_{1-2} \mathrm{~A}_{x} \mathrm{O}_{2}\right.$ : $\mathrm{A}=\mathrm{V}, \mathrm{Cr}, \mathrm{Mn}, \mathrm{Fe}, \mathrm{Co}$ or $\mathrm{Ni}$ ). Gray solid lines: total DOS; black solid lines: dopant's DOS. The states are labeled (a)-(k). Reproduced from Ref. [430].

strategy. $\mathrm{TiO}_{2}$ in contact with other semiconducting materials forms a heterostructure, in which the mismatch of the band positions of the two differing materials gives rise to the formation of rectifying junctions. After photogeneration, hole and electron injection takes place between the valence and conduction bands of the two semiconductors, respectively. The process may greatly contribute to an efficient charge separation and inhibition of recombination. As such, the lifetime of generated electrons and holes can increase significantly, thus allowing chemical reactions to take place on the surface $[35,428]$.

In the case of semiconductors (e.g. $\mathrm{TiO}_{2}$ ) and metal nanoparticles being in contact, another type of rectifying interface known as Schottky junction forms. After photogeneration in $\mathrm{TiO}_{2}$, electrons from the conduction band inject to the Fermi level of the metal. A reverse electron transfer is limited by the Schottky barrier, thus the metal becomes negatively charged whereas holes accumulate in $\mathrm{TiO}_{2}$ making it positively charged. Since $\mathrm{TiO}_{2}$ is an n-type semiconductor having electron affinity of $3.9 \mathrm{eV}$, metals of large work function such as $\mathrm{Pt}(5.7 \mathrm{eV}), \mathrm{Pd}(5.1 \mathrm{eV})$, $\mathrm{Rh}(5.0 \mathrm{eV})$ and $\mathrm{Au}(5.4 \mathrm{eV})$ are particularly suitable for achieving high Schottky barriers (above $1 \mathrm{eV}$ ) and thus good electron-hole separation. Apart from suppressed carrier recombination, a further advantage of the metal- $\mathrm{TiO}_{2}$ heterostructures is the catalytic activity offered by the surface of the nanosized metal co-catalyst $[35,428,436]$.

\subsection{Water splitting}

Efficient water splitting by solar irradiation to produce renewable $\mathrm{H}_{2}$ fuel would be an ideal and ultimate solution for the quest of continuously growing energy demand of our civilization. The first report on photoelectrochemical splitting on $\mathrm{TiO}_{2}$ surfaces [437] by Fujishima and Honda initiated a boost of the field. Although $\mathrm{TiO}_{2}$ based materials have been studied for over 40 years to find highly efficient photocatalyst and photoelectrodes, it seems today, that other materials such as layered perovskites $\left(\mathrm{A}_{2} \mathrm{La}_{2} \mathrm{Ti}_{3} \mathrm{O}_{10}\right.$, where $\mathrm{A}$ is an alkali metal and their heterostructures), niobiates $\left(\mathrm{K}_{4} \mathrm{Nb}_{6} \mathrm{O}_{17}\right.$, $\left.\mathrm{Rb}_{4} \mathrm{Nb}_{6} \mathrm{O}_{17}, \mathrm{Ba}_{5} \mathrm{Nb}_{4} \mathrm{O}_{15}, \mathrm{Cs}_{2} \mathrm{Nb}_{4} \mathrm{O}_{11}\right)$, tantalates $\left(\mathrm{Ta}_{2} \mathrm{O}_{5}, \mathrm{La}\right.$ and Sr-doped $\mathrm{NaTaO}_{3}$ ) and their heterostructures with cocatalyst metals and metal oxides are more active in the reaction [426,438-442].

It is worth mentioning here that in the scientific literature, several papers claim that $\mathrm{TiO}_{2}$ based (and also other) photocatalysts are efficient for splitting water. In fact, this is a rather general and unfortunate misconception because of the sacrificial reducing (e.g. alcohols, aldehydes, ascorbic acid, sulfides, EDTA) or oxidizing agents (e.g. $\mathrm{Ag}^{+}, \mathrm{Fe}^{3+}$ ) that are applied in water to produce $\mathrm{H}_{2}$ or $\mathrm{O}_{2}$, respectively. In such a context, one shall not call the photocatalytic reaction as water splitting, since it is the easily oxidizable or reducible component of the solution which undergoes oxidation or reduction, not the water itself, as emphasized by Kudo and Miseki in their review paper "Water splitting means to split water into $\mathrm{H}_{2}$ and $\mathrm{O}_{2}$ in a stoichiometric amount in the absence of sacrificial reagents." which should be kept in mind to avoid confusing statements in scientific publications [438].

\subsection{Contaminant degradation}

Titania nanostructures and their chemically doped and cocatalyst decorated derivatives have been extensively studied in degrading organic impurities being present in water as well as on solid surfaces. While the electrons accumulated typically on the co-catalyst nanoparticles (Pt, Pd, Rh) are expected to interact with unsaturated and aromatic bonds in organic moieties, holes on the surface of $\mathrm{TiO}_{2}$ are responsible for the initiation of oxidative processes that may result in $\mathrm{C}-\mathrm{C}$ bond scission, dehydrogenation or alike $[372,421,443]$. In the presence of water (medium of reaction or surface adsorbed), moieties such as hydroxyl radicals, peroxides and superoxides form on the surface of illuminated titanias and titanates. Owing to the highly oxidative nature of these species, also the cell walls and membranes of microorganisms can be destroyed, thus inducing a truly antimicrobial effect (Fig. 70) [423,429,444-447].

Most of the photocatalytic material related studies focus on the degradation of organic dyes, which typically implies damage in the delocalized electron cloud of the compounds and a consequential change or loss of color. As such, the process is certainly suitable for esthetic treatment of waters or contaminated surfaces, however in most of the cases the fate of pollutants and the practical use of the materials remain questions. There is also only very limited information 

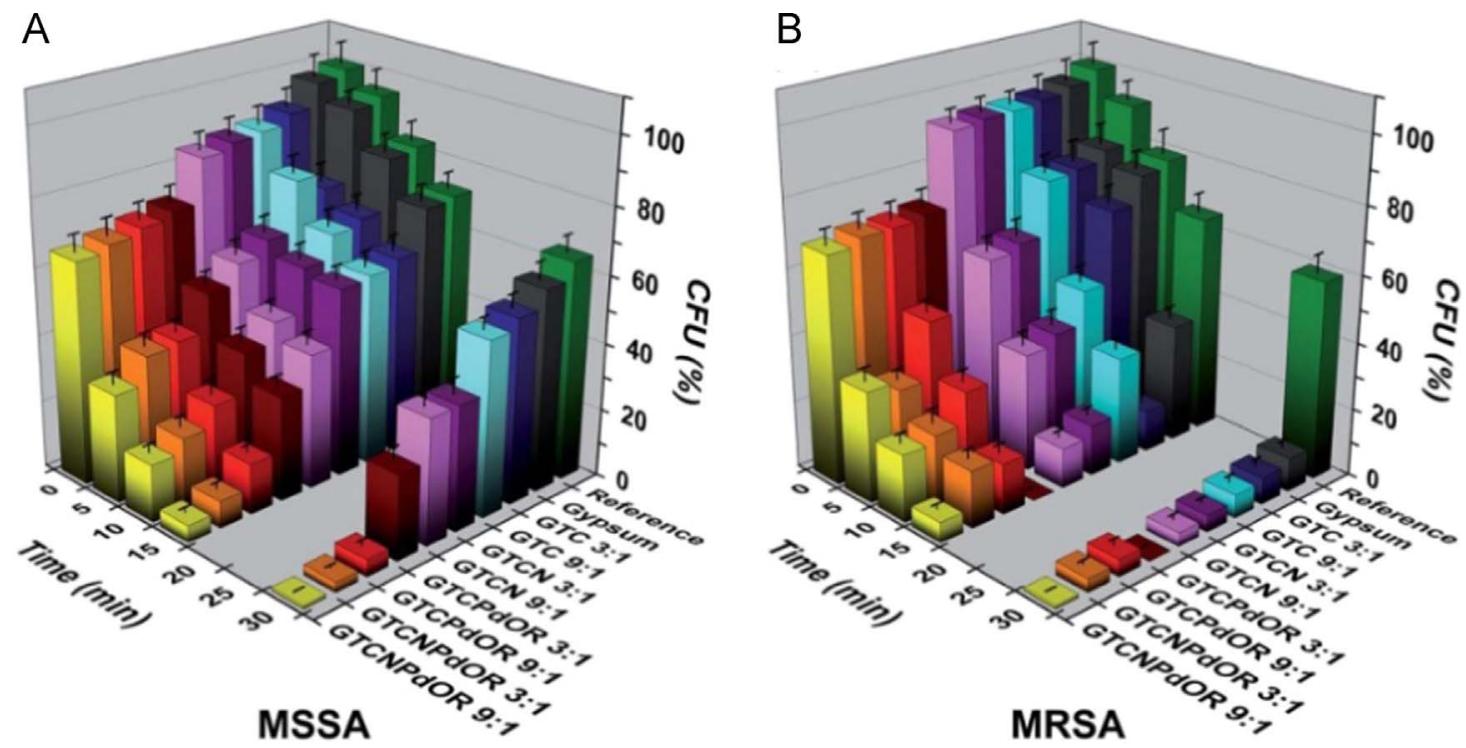

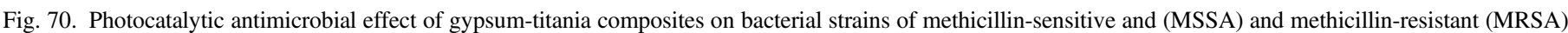

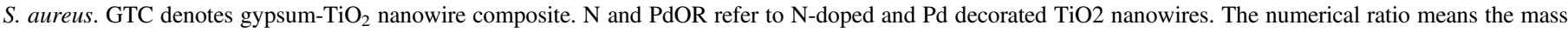
ratios for gypsum and titania in the samples. Reproduced from Ref. [423]

about the formed products after the decomposition of dangerous pollutants such as pesticides, antibiotics, hormones or other organics from waters as well as volatile organic compounds from air. Only papers published recently report on the follow up of product distributions after decomposition, and deal with complete mineralization of pollutants (i.e. oxidation to $\mathrm{CO}_{2}$ ). For practical applications, in fact, these latter studies are extremely important because with an incomplete photocatalytic treatment one may eventually produce a number of different smaller molecules that can even be potentially more dangerous than the known starting materials (Fig. 71) [448-453].

1D titanates and titanias perform similar (sometimes a bit better, sometimes worse) to their OD counterpart in most of the reported contaminant remediation and disinfection processes (Fig. 72). The major advantage however is the easier handling of filamentous nanoparticles (nanowires and nanotubes) as well as aligned mesoporous films of tubular of tubular nanostructures. The latter ones, obtained by electrochemical anodization, may be directly applied as solid photocatalytic surfaces, while from the filamentous nanostructures it is quite straightforward to filter entangled films when the filaments are suspended in e.g. water. Accordingly, for surface coatings, porous composites and membrane-type photocatalytic applications the 1D nanomaterials are often more practical than the OD nanoparticles $[35,423,454]$.

Another emerging and relevant application of $\mathrm{TiO}_{2}$ based photocatalysts is the mitigation of heavy metal cations $\left(\mathrm{Cr}^{6+}\right.$, $\mathrm{As}^{4+}, \mathrm{Fe}^{3+}, \mathrm{Cd}^{2+}, \mathrm{Cu}^{2+}$ ) from waters. The process is based on a photocatalytic reduction of the contaminant cations to lower their oxidation states, in which those are either less poisonous or can be precipitated and separated from the solution. To supply electrons for the reduction, sacrificial agents (i.e. hole scavengers) such as methanol, ethanol, 4chlorophenol and organic dyes are applied [455-464].

\subsection{Solar cells}

The pioneering work of O'Regan and Grätzel [465] towards novel photoelectrochemical devices based on $\mathrm{TiO}_{2}$ photoanodes initiated a whole new concept for solar cells. It was a paradigm change, since a highly promising competitor of the conventional Si based devices was born. In their novel solar cell, organic dye sensitized porous $\mathrm{TiO}_{2}$ films were applied as photoanode (Fig. 73). The photogenerated electrons inject from the dye to the conduction band of $\mathrm{TiO}_{2}$ and then through the collecting electrode feed current in an external circuit. Simultaneously, holes oxidize $\mathrm{I}^{-}$in the electrolyte $\left(3 \mathrm{I}^{-} \rightarrow \mathrm{I}_{3}^{-}\right)$. After diffusion of the oxidized species towards the counter electrode (typically Pt coated or decorated transparent conductive oxide), those undergo a reversed reaction (reduction) by the electrons returning to the cell from the external electrical circuit [466].

The efficiency of the solar cell is a function of dozens of parameters such as (i) geometry of the cell, (ii) chemistry of the electrolyte and dye, (iii) electrical conductivity, optical absorption, thickness and porosity of the photoanode, (iv) electrochemical properties of the interfaces and (v) electrical and optical properties of the transparent conductive electrodes, just to mention the most important ones [467]. Accordingly, in the past 25 years, an enormous research effort has been invested to optimize device operation thus improving solarto-electrical energy conversion efficiency, device reliability and lifetime as well as issues related to cost-reduction and mass-production. As compared to the first report, the solar-toelectric conversion efficiency has improved from the $\sim 8 \%$ up to $\sim 13 \%$ by now [468].

With the use of elongated nanoparticles in the electrode, the electrons need to pass much less particle-to-particle interfaces, thus minimizing trap-limited diffusion along the percolation path, i.e between the location of injection and the collector 
<smiles>Oc1nc(Cl)c(Cl)cc1Cl</smiles>

(1)<smiles>O=c1[nH]c(Cl)c(Cl)cc1Cl</smiles><smiles>CO[C@]1(O)C(Cl)=C(C)C(Cl)=C(Cl)N1[O]</smiles>

(3)<smiles>C[AlH]</smiles><smiles>O=C1N=C(Cl)C(Cl)=CC1Cl</smiles>

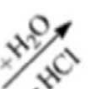<smiles>C=CC1(O)C#CC=C(Cl)NC1=O</smiles>

(4)

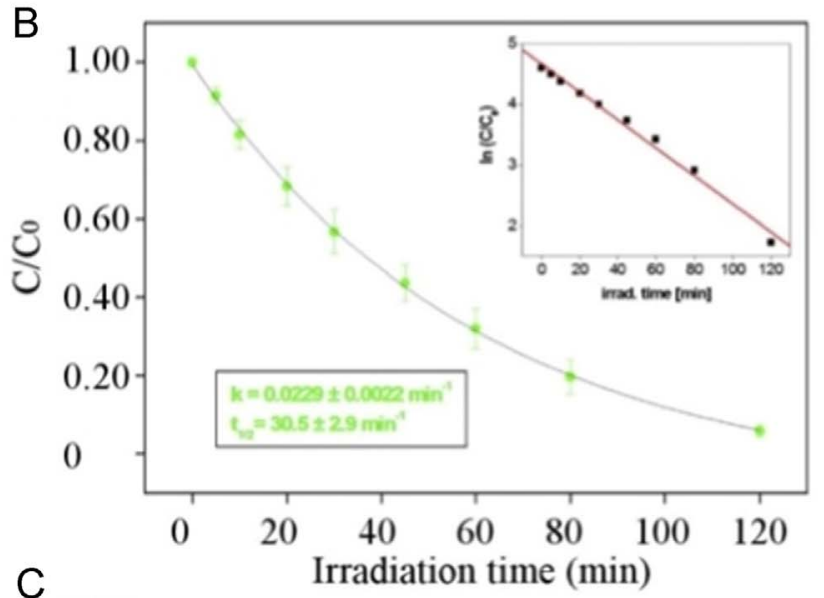<smiles>CCC1(O)C(Cl)=C(C)NC(=O)C1(CC)CC</smiles><smiles>O=C(O)c1cc(I)c(Cl)[nH]1</smiles>

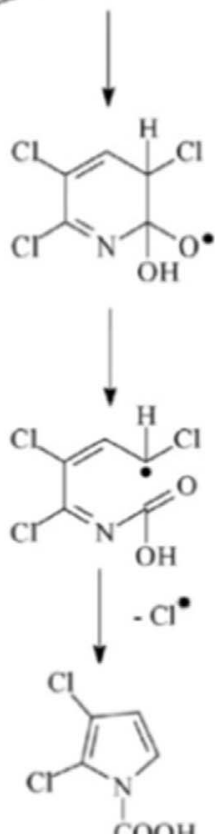

(6)

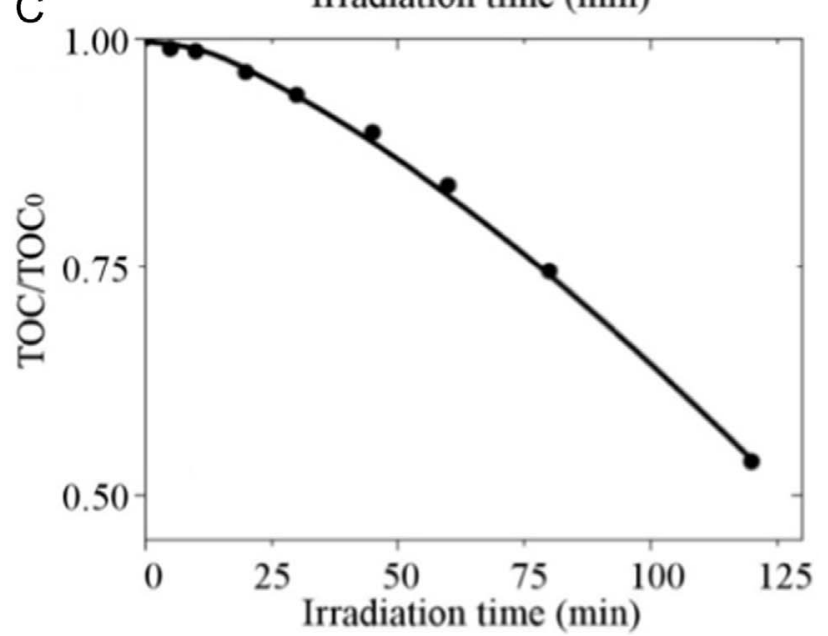

Fig. 71. (a) Decomposition paths of 3,5,6-trichloro-2-pyridinol (TCP) upon exposure with UV-A light. (b) Kinetics of photocatalytic decomposition for aqueous TCP. The inset shows a linearized plot, which suggestings first order kinetics. (c) Total organic content as a function of irradiation time. Note the differences between the concentration of the starting substance and total organic carbon in the solution. After 120 min irradiation, practically all TCP content has undergone decomposition, yet there is nearly $50 \%$ of total organic content in the form of various non-mineralized decomposition products. Reproduced from Ref. [450].
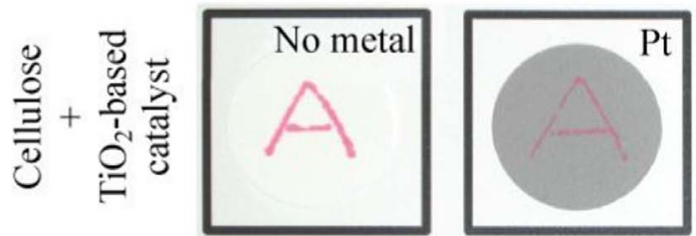

Before illumination

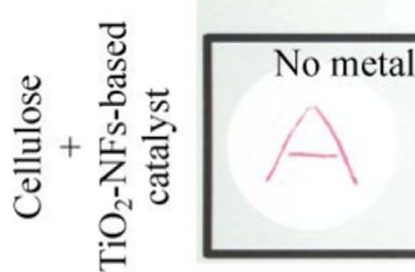

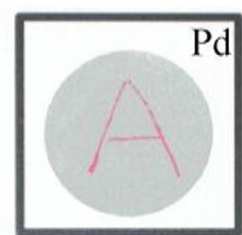

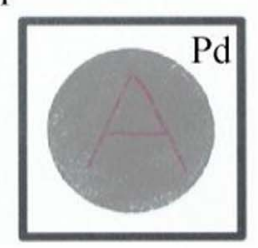

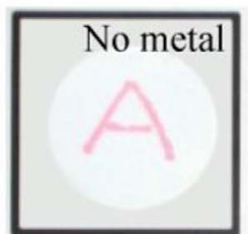
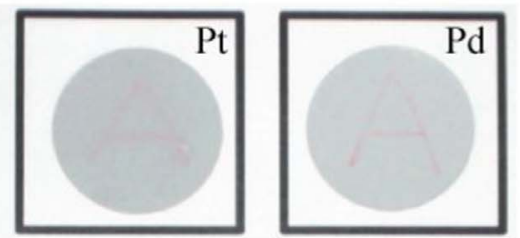

After 2 days of illumination
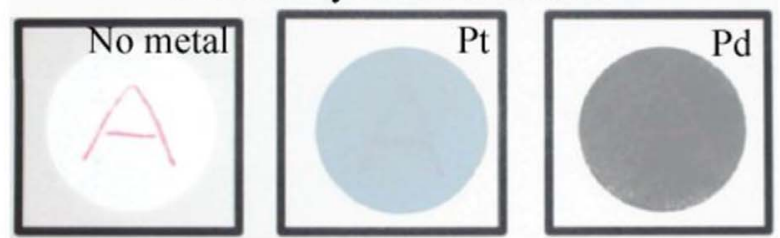

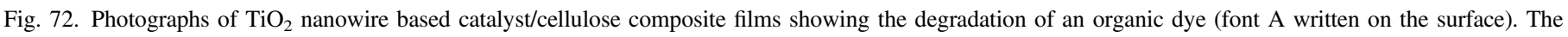

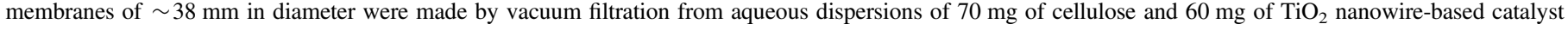
(pure $\mathrm{TiO}_{2}$ nanowire, or Pd or Pt decorated with metal content of $0.6 \mathrm{mg}$ ). Reproduced from Ref. [35]. 

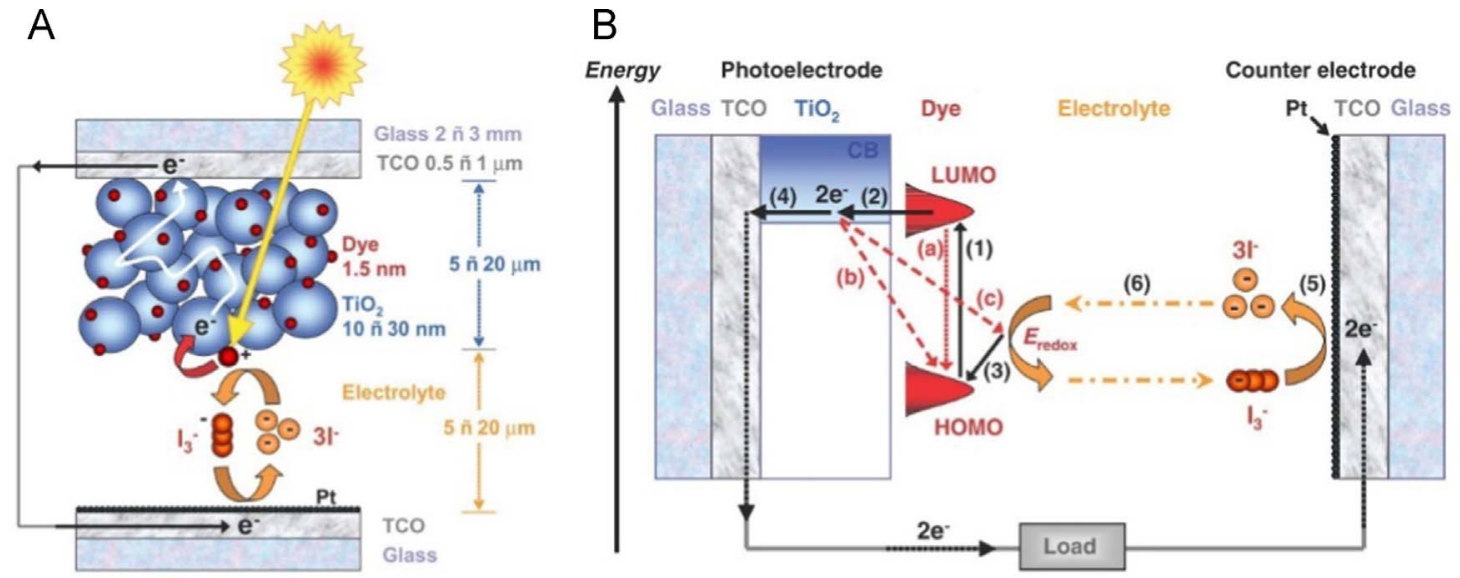

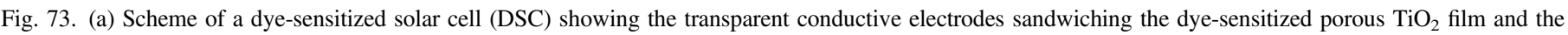

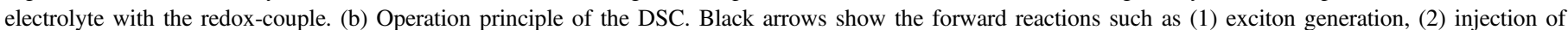

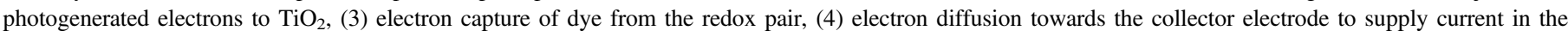

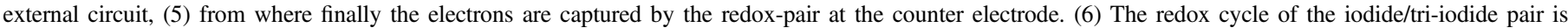

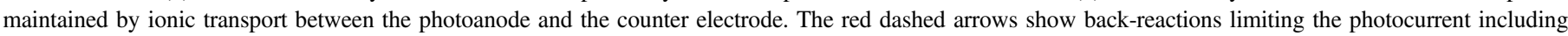

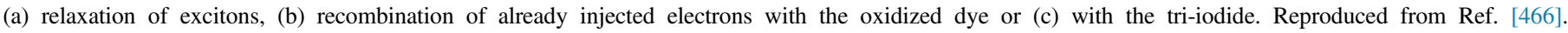
(For interpretation of the references to color in this figure legend, the reader is referred to the web version of this article.)

electrode [469-471]. Accordingly, phtotoanodes made of 1D nanomaterials are expected to outperform their OD counterparts. However, until now, the achieved best efficiency using electrospun or hydrothermally grown nanowires and nanorods or electrochemically etched nanotube arrays of $\mathrm{TiO}_{2}$ is $\sim 9 \%$ due to the limited specific surface area in reference to nanoparticle based components [472,473]. To improve the efficiency, a number of approaches are being researched nowadays. One alternative is a combination of nanoparticles and nanowires to form composites with improved specific surface area, yet having good percolation behavior [474-476]. Another very attractive route today is the application of perovskite-type absorbers such as $\mathrm{CH}_{3} \mathrm{NH}_{3} \mathrm{PbI}_{3}$ and mixed halide $\mathrm{CH}_{3} \mathrm{NH}_{3} \mathrm{PbI}_{3-x} \mathrm{Cl}_{x}$ instead of organic dyes [477] in contact with $\mathrm{TiO}_{2}$ thin films or porous structures, in which titania plays a similar role as in DSCs, i.e. helps in the separation of electrons and holes after photogeneration [478-482].

Despite the moderate efficiency of dye-sensitized $(\sim 13 \%)$ and perovskite solar cells $(\sim 21 \%)$ as compared to $\mathrm{Si}(\sim 28 \%)$ or multi-junction based devices $(\sim 46 \%)$ [483], the novel technologies are indeed very attractive due to the feasibility for mass-production (e.g. by roll-to-roll printing) of large-footprint area flexible panels at affordable price [484-486].

\subsection{Batteries}

Rapid and reversible $\mathrm{Li}^{+}$insertion (intercalation), high specific surface area as well as good ion and electrical conductivity in titanates make these materials a good choice for selecting as battery anodes [487-492]. Among the several possible titania phases, spinnels are considered particularly appealing due to their small volumetric change upon intercalation, which is important from the aspects of device reliability and lifetime. Layered titanates on the other hand show better transport behavior allowing high initial discharge and reversible capacity of $\sim 350 \mathrm{~mA} \mathrm{~h}^{-1}$ and $\sim 200 \mathrm{~mA} \mathrm{~h}$ $\mathrm{g}^{-1}$, respectively.

Aligned titanate forests grown directly on the surface of titanium foils under hydrothermal conditions in alkaline media have been demonstrated as particularly attractive materials for Li battery cathodes (Fig. 74). The inherent advantage of these titanates is the excellent direct contact with Ti metal. Also the ordered pore structure suggests easy ion transport from/to the electrodes, while the well-defined porous structure having mechanical integrity is expected to contribute to simplified device integration [493].

\section{Concluding remarks}

The current international trend (or hype) to synthesize, characterize and investigate nanomaterials has also embraced $\mathrm{TiO}_{2}$ and titanates. Among various semiconductors, titanium dioxide $\left(\mathrm{TiO}_{2}\right)$ as a photocatalyst has been researched excessively due to its advantages such as low cost, availability in excess, chemically and biologically stable character and possession of high oxidative potential. Due to its favorable electronic and optoelectronic properties, it has been widely applied in solar cells and photo-catalysts as well. However, improved properties are necessary to meet the highly demanding and complex requirements set for new materials. The prosperous development of titanium dioxide nanomaterials has thrived the investigation of a novel class of $\mathrm{TiO}_{2}$-based nanostructures: layered titanate materials. Layered titanate materials (nano-tubes, -wires, -rods and -sheets) have attractive features of their own including large ion-exchange capacity, fast ion diffusion and intercalation possibilities. These materials lend themselves quite well to building tiny structures in all sorts of sizes and shapes. Titanium-oxide nanostructures are almost invariably either amorphous or of the anatase phase. This provides good motivation for the surface scientist to find 

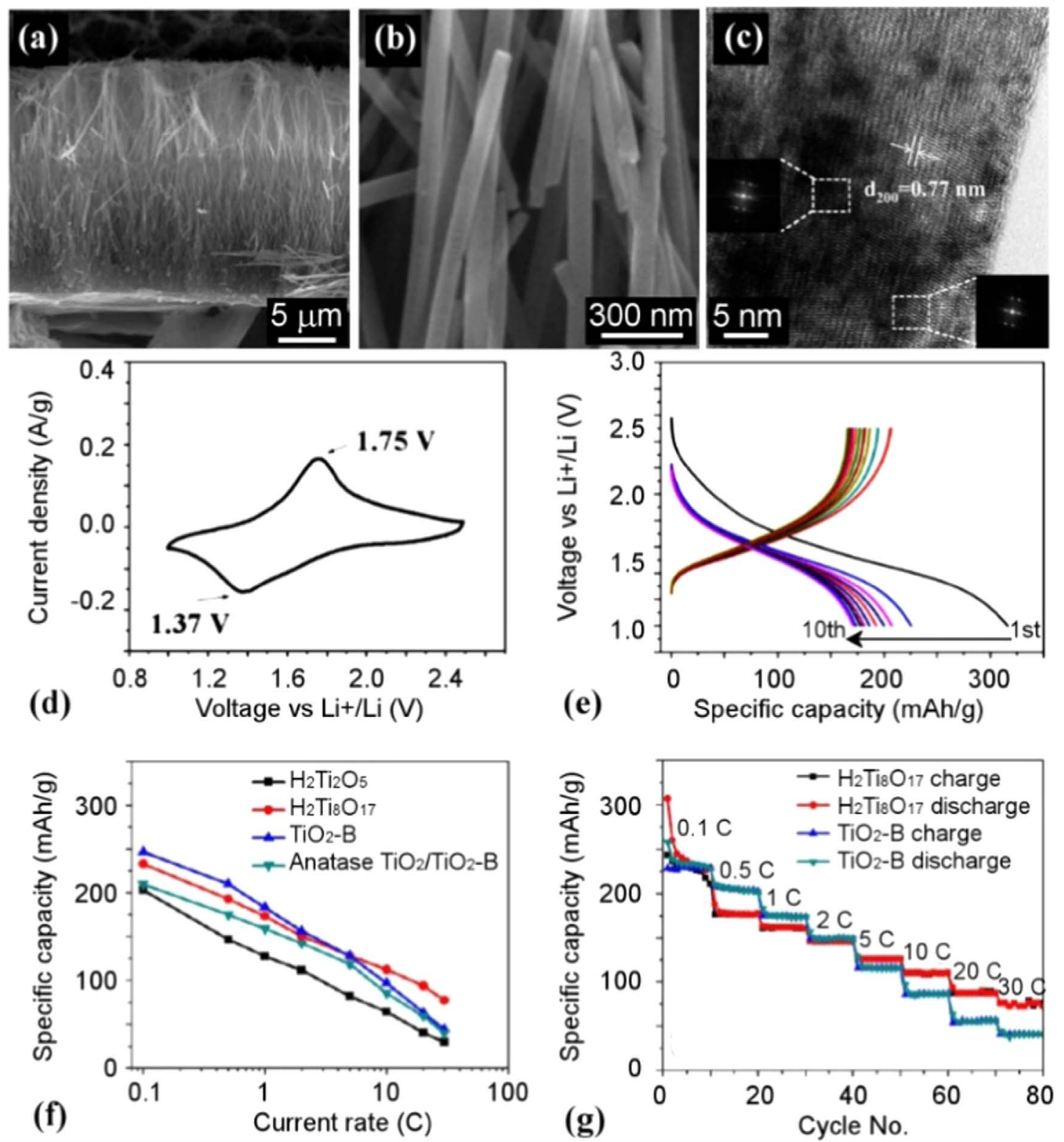

Fig. 74. Scanning (a), (b) and transmission (c) electron micrographs of $\mathrm{H}_{2} \mathrm{Ti}_{2} \mathrm{O}_{5}$ nanowires hydrothermally grown on Ti surface in $1 \mathrm{M} \mathrm{NaOH}$ aqueous solution at $220{ }^{\circ} \mathrm{C}$ for $24 \mathrm{~h}$ followed by soaking in $0.5 \mathrm{M} \mathrm{HCl}$ solution for $2 \mathrm{~h}$ to replace $\mathrm{Na}^{+}$of $\mathrm{Na}_{2} \mathrm{Ti}_{2} \mathrm{O}_{5}$ with $\mathrm{H}^{+}$. Panels (d) and (e) display the corresponding cyclic voltammetry and charge-discharge curves at $0.1 \mathrm{C}$ for the first ten cycles, respectively. (f) Specific capacities of titanate and titania based nanowire electrodes at different current rates; (g) the discharge/charge capacities of $\mathrm{H}_{2} \mathrm{Ti}_{8} \mathrm{O}_{17}$ and $\mathrm{TiO}_{2}-\mathrm{B}$ electrodes at different current rates; Reproduced from Ref. [493].

out more about the surface of these materials. This review highlights some of the significant insights obtained from molecular level studies of different titanate structures.

Several research groups have shown that the optical properties of titanates can be altered by surface modifiers. In most cases, modifications and doping dramatically change the structure due to ion exchange process. These new types of materials are good candidates mostly - but not exclusively for phototechnology applications. The modification of the optical properties of titanates often results simply from inclusion of the optical transitions of the surface modifier; however, in other cases the electronic states of titanates can also be affected by the modifier.
The fine structure of titanates are more complex and sensitive than the model systems of $\mathrm{TiO}_{2}$. Their structures and properties squeamishly depend on the preparation methods and the effect of application environments. This opens broad application possibilities for titanates.

When reviewing the current literature on a new subject, previous errors and omissions - natural consequences of science in the making - typically become clear. Even though much work has been done and significant results have been achieved, the surface chemistry of titanates is far from being so completely resolved today. Some of the results reviewed here might be of fundamental and practical interest. It is our hope that titanates will remain important materials for many years to 
come, and that this review will help in linking the fundamental and applied streams of research on these interesting nanomaterials.

\section{Acknowledgment}

The financial support of the NKFIH OTKA K 112531 and NN 110676 projects is acknowledged. The support received from the Academy of Finland (projects Suplacat and Optifu) is gratefully acknowledged.

\section{References}

[1] Q. Chen, W.Z. Zhou, G.H. Du, L.M. Peng, Adv. Mater. 14 (2002) 1208-1211.

[2] U. Diebold, Surf. Sci. Rep. 48 (2003) 53-229.

[3] M.A. Henderson, Surf. Sci. Rep. 66 (2011) 185-297.

[4] L. Wang, T. Sasaki, Chem. Rev. 114 (2014) 9455-9486.

[5] M.A. Peña, J.L.G. Fierro, Chem. Rev. 101 (2001) 1981-2018.

[6] P. Roy, S. Berger, P. Schmuki, Angew. Chem. Int. Ed. 50 (2011) 2904-2939.

[7] J.M. Macak, H. Tsuchiya, P. Schmuki, Angew. Chem. Int. Ed. 44 (2005) 2100-2102.

[8] Y. Zhang, Z. Jiang, J. Huang, L.Y. Lim, W. Li, J. Deng, D. Gong, Y. Tang, Y. Lai, Z. Chen, RSC Adv. (2015) 79479-79510.

[9] T. Kasuga, M. Hiramatsu, A. Hoson, T. Sekino, K. Niihara, Langmuir 14 (1998) 3160-3163.

[10] A.R. Armstrong, G. Armstrong, J. Canales, P.G. Bruce, J. Power Sources 146 (2005) 501-506.

[11] Y.K. Zhou, L. Cao, F.B. Zhang, B.L. He, H.L. Li, J. Electrochem. Soc. 150 (2003) A1246-A1249.

[12] J.R. Li, Z.L. Tang, Z.T. Zhang, Electrochem. Commun. 7 (2005) 894-899.

[13] G.H. Du, Q. Chen, P.D. Han, Y. Yu, L.M. Peng, Phys. Rev. B 67 (2003) 035323.

[14] B.L. Wang, Q. Chen, R.H. Wang, L.M. Peng, Chem. Phys. Lett. 376 (2003) 726-731.

[15] Z.Y. Yuan, B.L. Su, Colloids Surf. A 241 (2004) 173-183.

[16] B. Zhao, L. Lin, C. Chen, Y. Chai, D. He, Acta Chim. Sin. 71 (2013) 93-101.

[17] R. Menzel, A.M. Peiro, J.R. Durrant, M.S.P. Shaffer, Chem. Mater. 18 (2006) 6059-6068.

[18] M. Qamar, C.R. Yoon, H.J. Oh, D.H. Kim, J.H. Jho, K.S. Lee, W.J. Lee, H.G. Lee, S.J. Kim, Nanotechnology 17 (2006) 5922-5929.

[19] C.-K. Lee, C.-C. Wang, M.-D. Lyu, L.-C. Juang, S.-S. Liu, S.-H. Hung, J. Colloid Interface Sci. 316 (2007) 562-569.

[20] E. Morgado Jr., M.A.S. de Abreu, G.T. Moure, B.A. Marinkovic, P. M. Jardim, A.S. Araujo, Mat. Res. Bull. 42 (2007) 1748-1760.

[21] C.-K. Lee, M.-D. Lyu, S.-S. Liu, H.-C. Chen, J. Taiwan Inst. Chem. Eng. 40 (2009) 463-470.

[22] M.D. Hernandez-Alonso, S. Garcia-Rodriguez, B. Sanchez, J. M. Coronado, Nanoscale 3 (2011) 2233-2240.

[23] V. Thu Ha Thi, A. Hang Thi, T. Lien Thi, N. Tuyet Mai Thi, T. Thanh Thuy Thi, P. Minh Tu, D. Manh Hung, N. Dinh Lam, J. Mater. Sci. 49 (2014) 5617-5625.

[24] D.V. Bavykin, B.A. Cressey, M.E. Light, F.C. Walsh, Nanotechnology 19 (2008) 275604.

[25] H.Y. Zhu, Y. Lan, X.P. Gao, S.P. Ringer, Z.F. Zheng, D.Y. Song, J. C. Zhao, J. Am. Chem. Soc. 127 (2005) 6730-6736.

[26] M.A. Cortes-Jacome, G. Ferrat-Torres, L.F.F. Ortiz, C. Angeles-Chavez, E. Lopez-Salinas, J. Escobar, M.L. Mosqueira, J.A. Toledo-Antonio, Catal. Today, 126 (2007) 248-255.

[27] G. Li, Z.-Q. Liu, J. Lu, L. Wang, Z. Zhang, Appl. Surf. Sci. 255 (2009) 7323-7328.
[28] T. Shokuhfar, Q. Gao, A. Ashtana, K. Walzack, P. Heiden, C. Friedrich, J. Appl. Phys. 108 (2010) 104310.

[29] H. Xu, C. Li, D. He, Y. Jinag, Powder Diffr. 29 (2014) 147-150.

[30] B. Poudel, W.Z. Wang, C. Dames, J.Y. Huang, S. Kunwar, D.Z. Wang, D. Banerjee, G. Chen, Z.F. Ren, Nanotechnology 16 (2005) 1935-1940.

[31] G. Kozma, Z. Konya, A. Kukovecz, RSC Adv. 3 (2013) 7681-7683.

[32] D.V. Bavykin, F.C. Walsh, Titanate and Titania Nanotubes: Synthesis, Properties and Applications, RSC Publishing, Cambridge, 2010.

[33] K. Kordas, M. Mohl, Z. Kónya, Á. Kukovecz, Transl. Mater. Res. 2 (2015) 015003.

[34] T.P. Feist, P.K. Davies, J. Solid State Chem. 101 (1992) 275-295.

[35] M.-C. Wu, A. Sápi, A. Avila, M. Szabó, J. Hiltune, M. Huuhtane, G. Tóth, Á. Kukovecz, Z. Kónya, R. Keiski, W.-F. Su, H. Jantunen, K. Kordás, Nano Res. 4 (2011) 360-369.

[36] T. Beuvier, M. Richard-Plouet, L. Brohan, J. Phys. Chem. C 113 (2009) 13705-13716.

[37] S. Zhang, Q. Chen, L.M. Peng, Phys. Rev. B 71 (2005) 014104.

[38] E. Horváth, A. Kukovecz, Z. Kónya, I. Kiricsi, Chem. Mater. 19 (2007) 927-931.

[39] A. Bo, H. Zhan, J. Bell, H. Zhu, Y. Gu, RSC Adv. 4 (2014) 56970-56976.

[40] M. Chang, C.C. Chung, J.R. Deka, C.H. Lin, T.W. Chung, Nanotechnology 19 (2008) 025710.

[41] J.E. McCarthy, M. Bent, R. Blake, Y.K. Gunko, E. Horváth, Z. Kónya, A. Kukovecz, I. Kiricsi, J.N. Coleman, J. Mater. Chem. 17 (2007) 2351-2358.

[42] I.A. Santos-Lopez, B.E. Handy, R. Garcia de Leon, Thermochim. Acta 567 (2013) 85-92.

[43] D. Xu, J. Li, Y. Yu, J. Li, Sci. China-Chem. 55 (2012) 2334-2345.

[44] W. Ming-Chung, G. Toth, A. Sapi, A.R. Leino, Z. Kónya, A. Kukovecz, W.F. Su, K. Kordas, J. Nanosci. Nanotechnol. 12 (2012) 1421-1424.

[45] W. Zhou, H. Liu, R.I. Boughton, G. Du, J. Lin, J. Wang, D. Liu, J. Mater. Chem. 20 (2010) 5993-6008.

[46] T. Gao, Q. Wu, H. Fjellvag, P. Norby, J. Phys. Chem. C 112 (2008) $8548-8552$.

[47] D.V. Bavykin, S.N. Gordeev, A.V. Moskalenko, A.A. Lapkin, F. C. Walsh, J. Phys. Chem. B 109 (2005) 8565-8569.

[48] B. Wang, Y. Shi, D. Xue, J. Solid State Chem. 180 (2007) 1028-1037.

[49] N. Viriya-Empikul, N. Sano, T. Kikuchi, S. Bureekaew, W. Tanthapanichakoon, T. Charinpanitkul, J. Ind. Eng. Chem. 16 (2010) 63-67.

[50] Q. Li, T. Kako, J. Ye, Appl. Catal. A 375 (2010) 85-91.

[51] Z.R. Tang, F. Li, Y. Zhang, X. Fu, Y.J. Xu, J. Phys. Chem. C 115 (2011) $7880-7886$.

[52] P. Pusztai, R. Puskas, E. Varga, A. Erdohelyi, A. Kukovecz, Z. Kónya, J. Kiss, Phys. Chem. Chem. Phys. 16 (2014) 26786-26797.

[53] A. Oszkó, G. Pótári, A. Erdőhelyi, A. Kukovecz, Z. Kónya, I. Kiricsi, J. Kiss, Vacuum 85 (2011) 1114-1116.

[54] A. Riss, M.J. Elser, J. Bernardi, O. Diwald, J. Am. Chem. Soc. 131 (2009) 6198-6206.

[55] D. Wang, F. Zhou, C. Wang, W. Liu, Micropor. Mesopor. Mater. 116 (2008) 658-664.

[56] H.G. Yu, J.G. Yu, B. Cheng, M.H. Zhou, J. Solid State Chem. 179 (2006) 349-354.

[57] C.H. Lin, D.S.H. Wong, S.Y. Lu, ACS Appl. Mater. Interf. 6 (2014) 16669-16678.

[58] J. Wang, S. Yin, T. Sato, Mat. Sci. Eng. C 126 (2006) 53-58.

[59] A. Thorne, A. Kruth, D. Tunstall, J.T.S. Irvine, W.Z. Zhou, J. Phys. Chem. B 109 (2005) 5439-5444.

[60] T. Dittrich, J. Weidmann, V.Y. Timoshenko, A.A. Petrov, F. Koch, M. G. Lisachenko, E. Lebedev, Mat. Sci. Eng. B 69 (2000) 489-493.

[61] S. Li, L. Liu, D. Tian, H. Zhao, Micro Nano Lett. 6 (2011) 233-235.

[62] A. Matsuda, H. Sakamoto, M.A.B. Mohd Nor, G. Kawamura, H. Muto, J. Phys. Chem. B 117 (2013) 1724-1730.

[63] D. Madarász, I. Szenti, L. Nagy, A. Sápi, A. Kukovecz, Z. Kónya, Adsorpt. - J. Int. Adsorpt. Soc. 19 (2013) 695-700.

[64] M. Kitano, K. Nakajima, J.N. Kondo, S. Hayashi, M. Hara, J. Am. Chem. Soc. 132 (2010) 6622-6623. 
[65] E. Wada, M. Kitano, K. Nakajima, M. Hara, J. Mater. Chem. A 1 (2013) $12768-12774$.

[66] M. Kitano, E. Wada, K. Nakajima, S. Hayashi, S. Miyazaki, H. Kobayashi, M. Hara, Chem. Mater. 25 (2013) 385-393.

[67] E. Horvath, I. Szilagyi, L. Forro, A. Magrez, J. Colloid Interface Sci. 416 (2014) 190-197.

[68] E. Morgado Jr., P.M. Jardim, B.A. Marinkovic, F.C. Rizzo, M.A.S. De Abreu, J.L. Zotin, A.S. Araujo, Nanotechnology 18 (2007) 495710.

[69] Y. Liu, J. Liu, W. Yao, W. Cen, H. Wang, X. Weng, Z. Wu, RSC Adv. 3 (2013) 18803-18810.

[70] E. Morgado Jr., M.A.S. de Abreu, O.R.C. Pravia, B.A. Marinkovic, P. M. Jardim, F.C. Rizzo, A.S. Araujo, Solid State Sci. 8 (2006) 888-900.

[71] D.V. Bavykin, F.C. Walsh, J. Phys. Chem. C 111 (2007) 14644-14651.

[72] M. Yada, Y. Inoue, I. Noda, T. Morita, T. Torikai, T. Watari, T. Hotokebuchi, J. Nanomater. 476585 (2013).

[73] E. Horváth, Preparation and Characterization of a Multifunctional Polymer Reinforcement Nanomaterial (Doctoral thesis), University of Szeged, 2009.

[74] H. Niu, Y. Cai, Y. Shi, F. Wei, S. Mou, G. Jiang, J. Chromatogr. A 1172 (2007) 113-120.

[75] R. Ma, T. Sasaki, Y. Bando, J. Am. Chem. Soc. 126 (2004) $10382-10388$.

[76] D.H. Kim, K.S. Lee, J.H. Yoon, J.S. Jang, D.-K. Choi, Y.-K. Sun, S.J. Kim, K.S. Lee, Appl. Surf. Sci. 254 (2008) 7718-7722.

[77] I. Brnardic, M. Huskic, P. Umek, A. Fina, T.H. Grguric, Phys. Stat. Solidi A 210 (2013) 2284-2291.

[78] M. Pedroni, F. Piccinelli, S. Polizzi, A. Speghini, M. Bettinelli, P. HaroGonzalez, Mater. Lett. 80 (2012) 81-83.

[79] M. Huskic, T.H. Grguric, P. Umek, I. Brnardic, Polym. Compos. 34 (2013) 1382-1388.

[80] Y. Lai, Y. Tang, J. Huang, H. Wang, H. Li, D. Gong, X. Ji, J. Gong, C. Lin, L. Sunb, Z. Chen, Soft Matter 7 (2011) 6313-6319.

[81] J.R.E. Barker, C.R. Thomas, J. Appl. Phys. 35 (1964) 3203-3215.

[82] J.H. Anderson, G.A. Parks, J. Phys. Chem. 72 (1968) 3662-3668.

[83] M. Nilsson, M. Strømme, J. Phys. Chem. B 109 (2005) 5450-5455.

[84] S. Bone, J. Eden, R. Pethig, Int. J. Quantum Chem. 20 (1981) 307-316.

[85] H. Morgan, R. Pethig, Int. J. Quantum Chem. 26 (1984) 209-216.

[86] R. Pethig, Ferroelectrics 86 (1988) 31-39.

[87] I. Pochard, S. Frykstrand, O. Ahlström, J. Forsgren, M. Strømme, J. Appl. Phys. 115 (2014) 044306.

[88] C. Cramer, S. De, M. Schönhoff, Phys. Rev. Lett. 107 (2011) 028301.

[89] J.S. Dryden, R.J. Meakins, Discuss. Faraday Soc. 23 (1957) 39-49.

[90] N. Kamaya, K. Homma, Y. Yamakawa, M. Hirayama, R. Kanno, M. Yonemura, T. Kamiyama, Y. Kato, S. Hama, K. Kawamoto, A. Mitsui, Nat. Mater. 10 (2011) 682-686.

[91] I. Riess, Solid State Ion. 157 (2003) 1-17.

[92] H. Haspel, N. Laufer, V. Bugris, R. Ambrus, P. Szabó-Révész, Á. Kukovecz, J. Phys. Chem. C 116 (2012) 18999-19009.

[93] S. Bone, R. Pethig, Int. J. Quantum Chem. 24 (1983) 133-141.

[94] S. Bone, BBA Protein Struct. M 1078 (1991) 336-338.

[95] J. Behi, S. Bone, H. Morgan, R. Pethig, Int. J. Quantum Chem. 22 (1982) $367-374$.

[96] T. Seiyama, N. Yamazoe, H. Arai, Sens. Actuator 4 (1983) 85-96.

[97] E. Traversa, Sens. Actuat. B - Chem. 23 (1995) 135-156.

[98] N. Barsan, U. Weimar, J. Electroceram. 7 (2001) 143-167.

[99] Z. Chen, C. Lu, Sens. Lett. 3 (2005) 274-295.

[100] A. Zahab, L. Spina, P. Poncharal, C. Marlière, Phys. Rev. B 62 (2000) 10000-10003.

[101] J.-W. Han, B. Kim, N.P. Kobayashi, J. Li, M. Meyyappan, Appl. Phys. Lett. 101 (2012) 142110.

[102] J.-W. Han, B. Kim, J. Li, M. Meyyappan, J. Phys. Chem. C 116 (2012) 22094-22097.

[103] J.J. Fripiat, A. Jelli, G. Poncelet, J. André, J. Phys. Chem. 69 (1965) 2185-2197.

[104] J.J. Fripiat, C. Van der Meersche, R. Touillaux, A. Jelli, J. Phys. Chem. 74 (1970) 382-393.

[105] G. Clement, H. Knözinger, W. Stählin, B. Stübner, J. Phys. Chem. 83 (1979) 1280-1285.
[106] C. Yamahata, D. Collard, T. Takekawa, M. Kumemura, G. Hashiguchi, H. Fujita, Biophys. J. 94 (2008) 63-70.

[107] A. Soffer, M. Folman, Trans. Faraday Soc. 62 (1966) 3559-3569.

[108] A. Miller, E. Abrahams, Phys. Rev. 120 (1960) 745-755.

[109] V. Ambegaokar, B.I. Halperin, J.S. Langer, Phys. Rev. B 4 (1971) 2612-2620.

[110] A. Szent-Gyorgyi, Nature 148 (1941) 157-159.

[111] D.D. Eley, G.D. Parfitt, M.J. Perry, D.H. Taysum, Trans. Faraday Soc. 49 (1953) 79-86.

[112] D.D. Eley, G.D. Parfitt, Trans. Faraday Soc. 51 (1955) 1529-1539.

[113] M.H. Cardew, D.D. Eley, Discuss. Faraday Soc. 27 (1959) 115-128.

[114] D.D. Eley, D.I. Spivey, Trans. Faraday Soc. 56 (1960) 1432-1442.

[115] D.D. Eley, D.I. Spivey, Trans. Faraday Soc. 58 (1962) 411-415.

[116] S. Baxter, Trans. Faraday Soc. 39 (1943) 207-214.

[117] B. Rosenberg, J. Chem. Phys. 36 (1962) 816-823.

[118] M.G. Evans, J. Gergely, Biochim. Biophys. Acta 3 (1949) 188-197.

[119] N. Riehl, Kolloid Z. 151 (1957) 66-72.

[120] D.D. Eley, D.I. Spivey, Nature 188 (1960) 725.

[121] B. Rosenberg, Nature 193 (1962) 364-365.

[122] C. Filipič, A. Levstik, Z. Kutnjak, P. Umek, D. Arčon, J. Appl. Phys. 101 (2007) 084308.

[123] Z. Kutnjak, D. Vrbanić, S. Pejovnik, D. Mihailović, J. Appl. Phys. 99 (2006) 064311.

[124] D. Vrbanic, S. Pejovnik, D. Mihailovic, Z. Kutnjak, J. Eur. Ceram. Soc. 27 (2007) 975-977.

[125] Z. Kutnjak, C. Filipič, R. Podgornik, L. Nordenskiöld, N. Korolev, Phys. Rev. Lett. 90 (2003) 098101.

[126] Z. Kutnjak, G. Lahajnar, C. Filipič, R. Podgornik, L. Nordenskiöld, N. Korolev, A. Rupprecht, Phys. Rev. E 71 (2005) 041901.

[127] P. Sheng, Phys. Rev. B 21 (1980) 2180-2195.

[128] J.M.I. Leveritt, C. Dibaya, S. Tesar, R. Shrestha, A.L. Burin, J. Chem. Phys. 131 (2009) 245102-245107.

[129] B. Skinner, M.S. Loth, B.I. Shklovksii, Phys. Rev. E 80 (2009) 041925.

[130] I. Brovchenko, A. Krukau, A. Oleinikova, A.K. Mazur, J. Am. Chem. Soc. 130 (2007) 121-131.

[131] D.V. Bavykin, M. Carravetta, A.N. Kulak, F.C. Walsh, Chem. Mater. 22 (2010) 2458-2465.

[132] N. Yamazoe, J. Fuchigami, M. Kishikawa, T. Seiyama, Surf. Sci. 86 (1979) 335-344.

[133] S. Levy, M. Folman, J. Phys. Chem. 67 (1963) 1278-1283.

[134] J.J. Thomson, Philos. Mag. 5 (1893) 313-327.

[135] J.J. Fripiat, F. Toussaint, J. Phys. Chem. 67 (1963) 30-36.

[136] M.I. Cruz, W.E.E. Stone, J.J. Fripiat, J. Phys. Chem. (1972) 3078-3088.

[137] N. Bibent, A. Mehdi, G. Silly, F. Henn, S. Devautour-Vinot, Eur. J. Inorg. Chem. 21 (2011) 3214-3225.

[138] M.J. Cánovas, I. Sobrados, J. Sanz, J.L. Acosta, A. Linares, J. Membr. Sci. 280 (2006) 461-469.

[139] E. Jaimez, R.C.T. Slade, J. Chem. Soc. Dalton Trans. (1997) $1435-1440$.

[140] G. Alberti, L. Boccali, M. Casciola, L. Massinelli, E. Montoneri, Solid State Ion. 84 (1996) 97-104.

[141] M. Casciola, U. Costantino, F. Marmottini, Solid State Ion. 35 (1989) $67-71$.

[142] M. Casciola, S. Chieli, U. Costantino, A. Peraio, Solid State Ion. 46 (1991) 53-59.

[143] A. Shigematsu, T. Yamada, H. Kitagawa, J. Am. Chem. Soc. 133 (2011) 2034-2036.

[144] G. Careri, M. Geraci, A. Giansanti, J.A. Rupley, Proc. Natl. Acad. Sci. 82 (1985) 5342-5346.

[145] K.-D. Kreuer, Chem. Mater. 8 (1996) 610-641.

[146] C. Knight, G.A. Voth, Acc. Chem. Res. 45 (2011) 101-109.

[147] J. Bruinink, J. Appl. Electrochem. 2 (1972) 239-249.

[148] W. Saenger, C. Betzel, B. Hingerty, G.M. Brown, Nature 296 (1982) $581-583$.

[149] L.B. Nichols, J.M. Thorp, Trans. Faraday Soc. 66 (1970) 1741-1747.

[150] E.J. Murphy, Phys. Rev. 79 (1950) 396-397.

[151] X.L. Hu, J. Klimes, A. Michaelides, Phys. Chem. Chem. Phys. 12 (2010) 3953-3956. 
[152] G.A. Ludueña, T.D. Kühne, D. Sebastiani, Chem. Mater. 23 (2011) 1424-1429.

[153] A. Motta, M.P. Gaigeot, D. Costa, J. Phys. Chem. C 116 (2012) $12514-12524$.

[154] J.M. Douillard, G. Maurin, F. Henn, S. Devautour-Vinot, J.C. Giuntini, J. Colloid Interface Sci. 306 (2007) 440-448.

[155] M.A. Cook, R.O. Daniels, J.H. Hamilton, J. Phys. Chem. 58 (1954) $358-362$.

[156] E. McCafferty, V. Pravdic, A.C. Zettlemoyer, Trans. Faraday Soc. 66 (1970) 1720-1731.

[157] S. Cerveny, S. Arrese-Igor, J.S. Dolado, J.J. Gaitero, A. Alegria, J. Colmenero, J. Chem. Phys. 134 (2011) 034509-9.

[158] M. Monasterio, H. Jansson, J.J. Gaitero, J.S. Dolado, S. Cerveny, J. Chem. Phys. 139 (2013) 164714.

[159] V.P. Tomaselli, M.H. Shamos, Biopolymers 13 (1974) 2423-2434.

[160] D.N. Stamires, J. Chem. Phys. 36 (1962) 3174-3181.

[161] G.H. Bardelmeyer, Biopolymers 12 (1973) 2289-2302.

[162] S.M. Nelson, H.H. Huang, L.E. Sutton, Trans. Faraday Soc. 65 (1969) 225-243.

[163] H. Belarbi, A. Haouzi, J.C. Giuntini, S. Devautour-Vinot, M. Kharroubi, F. Henn, J. Non-Cryst. Solids 356 (2010) 664-668.

[164] S. Balme, M. Kharroubi, A. Haouzi, F. Henn, J. Phys. Chem. C 114 (2010) 9431-9438.

[165] P.A.M. Steeman, J.F.H. Baetsen, F.H.J. Maurer, Polym. Eng. Sci. 32 (1992) 351-356.

[166] P.M. Suherman, P. Taylor, G. Smith, J. Non-Cryst. Solids 305 (2002) $317-321$.

[167] P. Suherman, P. Taylor, G. Smith, Pharm. Res. 19 (2002) 337-344.

[168] Y. Kuroda, T. Watanabe, Y. Yoshikawa, R. Kumashiro, H. Hamano, M. Nagao, Langmuir 13 (1997) 3823-3826.

[169] I.R. Beattie, A. Dyer, Trans. Faraday Soc. 53 (1957) 61-66.

[170] A. Spanoudaki, B. Albela, L. Bonneviot, M. Peyrard, Eur. Phys. J. E 17 (2005) 21-27.

[171] P.M. Suherman, S. Geoff, J. Phys. D Appl. Phys. 36 (2003) 336-343.

[172] P. Pissis, J. Laudat, D. Daoukaki, A. Kyritsis, J. Non-Cryst. Solids 171 (1994) 201-207.

[173] H. Haspel, V. Bugris, Á. Kukovecz, Langmuir 30 (2014) 1977-1984.

[174] M.G. Evans, M. Polanyi, Trans. Faraday Soc. 32 (1936) 1333-1360.

[175] A.S. Nowick, W.K. Lee, H. Jain, Solid State Ion. 28-30 (1988) 89-94.

[176] S. Cerveny, G.A. Schwartz, J. Otegui, J. Colmenero, J. Loichen, S. Westermann, J. Phys. Chem. C 116 (2012) 24340-24349.

[177] H. Sugimoto, T. Miki, K. Kanayama, M. Norimoto, J. Non-Cryst. Solids 354 (2008) 3220-3224.

[178] L. Liu, Q.-X. Guo, Chem. Rev. 101 (2001) 673-696.

[179] W. Meyer, H. Neldel, Z. Techn. Phys. 12 (1937) 588.

[180] W. Meyer, H. Neldel, Phys. Z. 38 (1937) 1014-1019.

[181] Á. Kukovecz, G. Pótári, A. Oszkó, Z. Kónya, A. Erdőhelyi, J. Kiss, Surf. Sci. 605 (2011) 1048-1055.

[182] J. Kiss, A. Oszkó, G. Pótári, A. Erdőhelyi, Vacuum 86 (2012) 594-598.

[183] G. Pótári, D. Madarász, L. Nagy, B. László, A. Sápi, A. Oszkó, A. Kukovecz, A. Erdőhelyi, Z. Kónya, J. Kiss, Langmuir 29 (2013) 3061-3072.

[184] E.K. Ylhainen, M.R. Nunes, A.J. Silvestre, O.C. Monteiro, J. Mater. Sci. 47 (2012) 4305-4312.

[185] U. Diebold, T.E. Madey, Surf. Sci. Spectra 4 (1998) 227-231.

[186] J.T. Mayer, U. Diebold, T.E. Madey, E. Garfunkel, J. Electr. Spectrosc. Relat. Phenom. 73 (1995) 1-11.

[187] L.S. Dake, R.J. Lad, Surf. Sci. Spectra 4 (1998) 232-245.

[188] G. Yu, H.G. Yu, B. Cheng, X.J. Zhai, J.C. Yu, W.K. Ho, J. Phys. Chem. B 107 (2003) 13871-13879.

[189] H.-H. Ou, S.-L. Lo, Sep. Purif. Technol. 58 (2007) 179-191.

[190] H. Berger, H. Tang, F. Lévy, J. Cryst. Growth 130 (1993) 108.

[191] M.A. Cortés-Jácome, G. Ferrat-Torres, L.F. Flores Oeriz, C. AngelesChávez, E. López-Salinas, J. Escobar, M.L. Mosqueira, J.A. ToledoAntonio, Catal. Today 126 (2007) 248-255.

[192] L. Óvári, J. Kiss, Appl. Surf. Sci. 252 (2006) 8624-8629.

[193] J. Kiss, P. Pusztai, L. Óvári, K. Baán, G. Merza, A. Erdőhelyi, A. Kukovecz, Z. Kónya, J. Surf. Sci. Nanotechnol. 12 (2014) 252-258.
[194] G.S. Kim, H.K. Seo, Y.S. Kim, H.S. Shina, J. Appl. Phys. 101 (2007) 024314.

[195] S. Södergren, H. Siegbahn, H. Rensmo, H. Lindström, A. Hagfeldt, S. E. Lindquist, J. Phys. Chem. B 101 (1997) 3087-3090.

[196] M. Henderson, M. Worly, J. Phys. Chem. 89 (1985) 1417-1423.

[197] A. Trovarelli, C. Mustazza, G. Dolcetti, F. Kaspar, M. Grazioni, Appl. Catal. 65 (1990) 129-142.

[198] H. Perron, J. Vandenborre, C. Domain, R. Drot, J. Roques, E. Simoni, J.-J. Ehrhardt, H. Catalette, Surf. Sci. 601 (2007) 518-527.

[199] R.L. Kurtz, R. Stockbauer, T.E. Madey, E. Roman, J.-L. De Segovia, Surf. Sci. 218 (1989) 178-200.

[200] W. Gopel, J.A. Anderson, D. Frankel, M. Jaehnig, K. Phillips, J. A. Schafer, G. Rocker, Surf. Sci. 139 (1984) 333-346.

[201] P. Frantz, H.I. Kim, S.V. Didziulis, S. Li, Z. Chen, S.S. Perry, Surf. Sci. 596 (2005) 144-162.

[202] A.A. Yakovenko, V.A. Yashin, A.E. Kovalev, E.E. Fesenko, Biophysics 47 (2002) 891-895.

[203] T.R. Hughes, H.M. White, J. Phys. Chem. 71 (1967) 2192-2201.

[204] O.P. Ferreira, A.G. Souza Filho, J. Mendez Filho, O.L. Alves, J. Braz. Chem. Soc. 17 (2006) 393-402.

[205] R. Lemus, J. Mol. Spectr. 225 (2004) 73-79.

[206] Y.V. Kolenko, K.A. Kovnir, A.I. Gavrilov, A.V. Garshev, J. Frantti, O. I. Lebedev, B.R. Churagulov, G. van Tendeloo, M. Yoshimura, J. Phys. Chem. B 110 (2006) 4030-4038.

[207] R. Ma, K. Fukuda, T. Sasaki, M. Osada, Y. Bando, J. Phys. Chem. B 109 (2005) 6210-6214.

[208] T. Ohsaka, F. Izumi, Y. Fujiki, J. Raman Spectrosc. 7 (1978) 321-324.

[209] Y.L. Du, Y. Deng, M.S. Zhang, J. Phys. Chem. Solids 67 (2006) 2405-2408.

[210] M.J. Scepanovic, M. Grujic-Brojcin, Z.D. Dohcevic-Mitrovic, Z V. Popovic, Sci. Sinter. 41 (2009) 67-73.

[211] M. Murakami, Y. Matsumoto, K. Nakajima, T. Makino, Y. Segawa, T. Chikyow, P. Ahmet, M. Kawasaki, H. Koinuma, Appl. Phys. Lett. 78 (2001) 2664-2666.

[212] D. Kurita, S. Ohta, K. Sugiura, K. Koumoto, J. Appl. Phys. 100 (2006) 096105.

[213] K.M. Glassford, J.R. Chelikowsky, Phys. Rev. B 46 (1992) 1284-1298.

[214] N. Satoh, T. Nakashima, K. Kamikura, K. Yamamoto, Nat. Nanotechnol. 3 (2008) 106-111.

[215] Y. Li, T.J. White, S.H. Lim, J. Solid State Chem. 177 (2004) 1372-1381.

[216] K. Madhusudan Reddy, C.V. Gopal Reddy, S.V. Manorama, J. Solid State Chem. 158 (2001) 180-186.

[217] N. Serpone, D. Lawles, R. Khairutditov, J. Phys. Chem. 99 (1995) 16646-16654.

[218] R. Beranek, H. Kisch, Photochem. Photobiol. Sci. 7 (2008) 40-48.

[219] J.I. Pankove, Optical Process in Semiconductors, Prentice-Hall Inc., Upper Saddle River, NJ, 1971.

[220] H. Tang, K. Prasad, R. Sanilines, P.E. Schmid, F. Lewy, J. Appl. Phys. 75 (1994) 2042-2047.

[221] Gy Halasi, I. Ugrai, F. Solymosi, J. Catal. 281 (2011) 309-317.

[222] S.G. Kumar, L.G. Devis, J. Phys. Chem. A 115 (2011) 13211-13241.

[223] K.M. Reddy, I.V. Manorama, A.R. Reddy, Mat. Chem. Phys. 78 (2003) 239-245.

[224] T. Sasaki, M. Watenabe, J. Phys. Chem. B 101 (1997) 10159-10161.

[225] T. Sasaki, Supermol. Sci. 5 (1998) 367-371.

[226] H. Sato, K. Ono, T. Sasaki, A.V. Yamagishi, J. Phys. Chem. B 107 (2003) 9824-9828.

[227] N. Sakai, Y. Ebina, K. Takada, T. Sasaki, J. Am. Chem. Soc. 126 (2004) $5851-5858$.

[228] G.K. Mor, O.K. Varghese, M. Paulose, K. Shankar, C.A. Grimes, Sol. Energy Mater. Sol. Cell 90 (2006) 2011-2075.

[229] O.K. Varghese, G.K. Mor, C.A. Grimes, M. Paulose, N. Mukherjee, J. Nanosci. Nanotechnol. 4 (2004) 733-737.

[230] M. Paulose, O.K. Varghese, G.K. Mor, C.A. Grimes, K.G. Ong, Nanotechnology 17 (2006) 398-402.

[231] J. Tauc, Mater. Res. Bull. 5 (1970) 721-729.

[232] H. Zhang, L. Cao, W. Liu, G. Su, Appl. Surf. Sci. 259 (2012) 610-615. 
[233] F. Cesano, S. Bertarione, M.J. Uddin, G. Agostini, D. Scarano, A. Zeccina, J. Phys. Chem. C 114 (2010) 169-178.

[234] V.C. Ferreira, M.R. Nunes, A.J. Silvestre, O.C. Monteiro, Mater. Chem. Phys. 142 (2013) 355-362.

[235] G.K. Mor, O.K. Varghese, M. Paulose, C.A. Grimes, Adv. Funct. Mater. 15 (2005) 1291-1296.

[236] W. Wang, J. Zhang, H. Huang, Z. Wu, Z. Zhang, Colloids Surf. A 317 (2008) 270-276.

[237] S.R. Leadley, J.F. Watts, J. Electron Spectrosc. 85 (1997) 107-121.

[238] W. Wang, J. Zhang, H. Huang, Z. Wu, Z. Zhang, Appl. Surf. Sci. 253 (2007) 5393-5399.

[239] M. Huskic, G. Holjevac, U. Polona, B. Ivan, Polym. Compos. 34 (2013) $1382-1388$

[240] G. Brusatin, M. Guglielmi, P. Innocenzi, A. Martucci, G. Battglin, S. Pelli, G. Righini, J. Non-Cryst. Solid 220 (1997) 202-209.

[241] R. Mendoza-Serna, P. Bosch, J. Padilla, V.H. Lara, J. Mendez-Vivar, J. Non-Cryst. Solid 217 (1997) 30-40.

[242] L. Wang, W. Liu, T. Wang, J. Ni, Chem. Eng. J. 225 (2013) 153-163.

[243] Y. Ide, M. Ogawa, Chem. Commun. 11 (2003) 1262-1263.

[244] Y. Liu, J. Liu, W. Yao, W. Cen, H. Wang, X. Weng, Z. Wu, RSC Adv. 3 (2013) 18803-18810.

[245] X. Sun, Y. Li, Chem. Eur. J 9 (2003) 2229-2238.

[246] D. Madarász, I. Szenti, A. Sápi, J. Halász, Á. Kukovecz, Z. Kónya, Chem. Phys. Lett. 591 (2014) 161-165.

[247] W. Schmidt, in: Inamuddin, M. Luqman (Eds.), Ion Exchange Theory I: Theory and Materials, Springer, 2012, pp. 277-298.

[248] T. Wen, Z. Zhao, C. Shen, J. Li, X. Tan, A. Zeb, X. Wang, A.-W. Xu, Sci. Rep. 6 (2016) 20920.

[249] D.J. Yang, Z.F. Zheng, H.Y. Zhu, H.W. Liu, X.P. Gao, Adv. Mater. 20 (2008) 2777-2781.

[250] R. Chakraborty, B. Sen, S. Chatterjee, P. Chattopadhyay, Radiochim. Acta 101 (2013) 33-36.

[251] M. Xu, G. Wei, S. Li, X. Niu, H. Chen, H. Zhang, M. Chubik, A. Gromov, W. Han, J. Nanosci. Nanotechnol. 12 (2012) 6374-6379.

[252] J. Lehto, A. Clearfield, J. Radioanal. Nucl. Chem. Lett. 118 (1987) $1-13$.

[253] Y. Ishikawa, S. Tsukimoto, K.S. Nakayama, N. Asao, Nano Lett. 15 (2015) 2980-2984.

[254] R. Ma, T. Sasaki, Y. Bando, Chem. Commun. (2005) 948-950.

[255] G. Busca, Catal. Today 41 (1998) 191-206.

[256] M.I. Zaki, M.A. Hasan, F. Al-Sagheer, L. Pasupulety, Colloid Surf. A 190 (2001) 261-274.

[257] R. Camposeco, S. Castillo, V. Mugica, I. Mejía-Centeno, J. Marín Role 242 (2014) 313-320.

[258] E. Morgado, B.A. Marinkovic, P.M. Jardim, M.A.S. de Abreu, Maria da Graça C. Rocha, P. Bargiela, Mater. Chem. Phys. 126 (2011) 118-127.

[259] D.V. Bavykin, A.A. Lapkin, P.K. Plucinski, L. Torrente-Murciano, J. M. Friedrich, F.C. Walsh, Top. Catal. 39 (2006) 151-160.

[260] G. Sheng, L. Ye, Y. Li, H. Dong, H. Li, X. Gao, Y. Huang, Chem. Eng. J. 248 (2014) 71-78.

[261] W. Liu, T. Wang, A.G.L. Bortwick, Y. Wang, X. Yin, X. Li, J. Ni, Sci. Total. Environ. 456-457 (2013) 171-180.

[262] N.M. dos Santos, J.M. Rocha, J.M.E. Matos, O.P. Ferreira, J.M. Filho, B.C. Viana, A.C. Oliviera, Appl. Catal. A: Gen. 454 (2013) 74-80.

[263] L. Song, L. Cao, J. Li, W. Liu, F. Zhang, L. Zhu, G. Su, J. Alloy. Compd. 509 (2011) 6061-6066.

[264] C. Balasanthiran, J.D. Hoefelmeyer, Chem. Commun. 50 (2013) $5721-5724$

[265] J. Joo, S.G. Kwon, T. Yu, M. Cho, J. Lee, J. Yoon, T. Hyeon, J. Phys. Chem. B 109 (2005) 15297-15302.

[266] W.-Q. Han, W. Ween, D. Yi, Z. Liu, M.M. Maye, L. Lewis, J. Hanson, O. Gang, J. Phys. Chem. C 111 (2007) 14339-14342.

[267] T.M.F. Marques, O.P. Ferreira, J.A.P. da Costa, K. Fujisawa, M. Terrones, B.C. Viana, J. Phys. Chem. Solids 87 (2015) 213-220.

[268] A. Honciuc, M. Laurin, S. Albu, M. Sobota, P. Schmuck, J. Libuda, Langmuir 26 (2010) 14014-14023.

[269] V. Ponec, Stud. Surf. Sci. Catal. 64 (1991) 117-157.
[270] L. Óvári, S. Krick Calderon, Y. Lykhach, J. Libuda, A. Erdőhelyi, C. Papp, J. Kiss, H.-P. Steinrück, J. Catal. 307 (2013) 132-139.

[271] Zs Ferencz, A. Erdőhelyi, K. Baán, A. Oszkó, L. Óvári, Z. Kónya, C. Papp, H.-P. Steinrück, J. Kiss, ACS Catal. 4 (2014) 1205-1218.

[272] S. Suvanto, T.A. Tapani, A. Pakkanen, L. Backman, Appl. Catal. A: Gen. 1177 (1999) 25-36.

[273] A. Beck, A. Horváth, A. Sárkány, L. Guczi, Curr. Appl. Phys. 6 (2006) 200-204.

[274] H. Khosravian, Z. Liang, A. Uhl, M. Trenary, R. Meyer, J. Phys. Chem. C 116 (2012) 11987-11993.

[275] R.C. Reul, C.H. Bartholomew, J. Catal. 85 (1984) 78-88.

[276] M.-M. Walz, M. Schirmer, F. Vollnhals, T. Lukasczyk, H.-P. Steinrück, H. Marbach, Angew. Chem. Int. Ed. 49 (2010) 4669-4673.

[277] K. Muthukumar, H.O. Jeschke, R. Valenti, E. Begun, J. Schwenk, F. Porrati, M. Huth, Beilstein J. Nanotechnol. 3 (2012) 546-555.

[278] P. Krüger, M. Petukhov, B. Domenichini, A. Berkó, S. Bourgeois, J. Phys. Chem. C 116 (2012) 10617-10622.

[279] Z. Majzik, N. Balázs, L. Robin, M. Petukhov, B. Domenichini, S. Bourgeois, A. Berkó, Vaccum 86 (2012) 623-625.

[280] D. Madarász, G. Pótári, A. Sápi, B. László, C. Csudai, A. Oszkó, Á. Kukovecz, A. Erdőhelyi, Z. Kónya, J. Kiss, Phys. Chem. Chem. Phys. 15 (2013) 15917-15925.

[281] J. Kiviaho, M.K. Niemelä, M. Reinikainen, T. Vaara, T.A. Pakkanen, J, Mol. Catal. A 121 (1997) 1-8.

[282] M.-C. Wu, G. Tóth, A. Sápi, Z. Kónya, Á. Kukovecz, W.-F. Su, K. Kordás, J. Nanosci. Nanotechnol. 12 (2012) 1421-1426.

[283] W. Rupilius, J.J. McCoy, M. Orchin, Ind. Eng. Chem. Prod. Res. Dev. 10 (1971) 142-145.

[284] M. van Boven, N. Alemdaroglu, J.M.L. Penninger, J. Organomet. Chem. 84 (1975) 65-74.

[285] S.C. Petitto, E.M. Marsh, G.A. Carson, M.A. Langell, J. Mol. Catal. A: Chem. 281 (2008) 49-58.

[286] Z. Zsoldos, L. Guczi, J. Phys. Chem. 96 (1992) 9393-9400.

[287] M.C. Biesinger, B.P. Pague, A.P. Grosvenor, L.W.M. Lau, A.R. Gerson, R. St, C. Smart, Appl. Surf. Sci. 257 (2011) 2717-2730.

[288] L. Guczi, G. Boskovic, E. Kiss, Catal. Rev. 52 (2010) 133-203.

[289] V.A.D. O'Shea, M.C.A. Galvan, A.E.P. Parts, J.M. Campos-Martin, J.L. G. Fierro, Chem. Commun. 47 (2011) 7131-7133.

[290] R.P. Galhenage, H. Yan, S.A. Tenny, N. Park, G. Henkelman, P. Albrecht, D.R. Mullins, D.A. Chen, J. Phys. Chem. C 117 (2013) 7191-7201.

[291] W.F. Egelhoff, Surf. Sci. Rep. 6 (1987) 253-415.

[292] C.R. Henry, Surf. Sci. Rep. 31 (1998) 231-325.

[293] C. Papp, H.-P. Steinrück, Surf. Sci. Rep. 68 (2013) 446-487.

[294] L. Zhou, S. Zhang, J. Cheng, L. Zhang, Z. Zheng, Mater. Sci. Eng. B 49 (1997) 117-122.

[295] D. Wu, Y. Chen, J. Liu, X. Zhao, A. Li, N. Ming, Appl. Phys. Lett. 87 (2005) 112501-112503.

[296] J. Jacimovic, E. Horváth, B. Náfrádi, R. Gaál, N. Nikseresht, APL Mater. 1 (2013) 032111-032115.

[297] D.V. Bavykin, J.M. Fridrich, F.C. Walsh, Adv. Mater. 18 (2006) $2807-2824$

[298] J.J. Wu, C.C. Yu, J. Phys. Chem. B 108 (2004) 3377-3379.

[299] L. Miao, Y. Ina, S. Tanemura, T. Jiang, M. Tanemura, K. Kaneko, S. Toh, Y. Mori, Surf. Sci. 601 (2007) 2792-2799.

[300] J.A. Toledo-Antonio, S. Capula, M.A. Cortes-Jacome, C. AngelesChavez, E. Lopez-Salinas, G. Ferrat, J. Navarrete, J. Escobar, J. Phys. Chem. C 111 (2007) 10799-10805.

[301] E.N. Gribov, D. Cocina, G. Spoto, S. Bordiga, G. Ricchiardi, A. Zecchina, Phys. Chem. Chem. Phys. 8 (2006) 1186-1196.

[302] K. Hadjiivanov, J. Lamotte, J.C. Lavalley, Langmuir 13 (1997) 3374-3381.

[303] S. Bordiga, G. Turnes Palomino, D. Arduino, C. Lamberti, A. Zecchina, C. Otero Areán, J. Mol. Catal.: Chem. 146 (1999) 97-106.

[304] M. Tóth, J. Kiss, A. Oszkó, G. Pótári, B. László, A. Erdőhelyi, Top. Catal. 55 (2012) 747-756.

[305] F. Solymosi, A. Erdőhelyi, J. Mol. Catal. 8 (1980) 471-474. 
[306] G. Poirier, G.E. Hance, J.M. White, J. Phys. Chem. 97 (1993) 5965-5972.

[307] A. Berkó, G. Ménesi, F. Solymosi, Surf. Sci. 372 (1997) 202-210.

[308] J. Evans, B.E. Hayden, M.A. Newton, Surf. Sci. 462 (2000) 169-180.

[309] A. Berkó, G. Ménesi, F. Solymosi, J. Phys. Chem. 100 (1996) 17732-17734.

[310] F. Solymosi, Catal. Rev. 1 (1968) 233-255.

[311] M.A. Vannice, R.L. Garten, J. Catal. 56 (1979) 236-248.

[312] F. Solymosi, A. Erdőhelyi, T. Bánsági, J. Catal. 68 (1981) 371-382.

[313] F. Solymosi, I. Tombácz, J. Koszta, J. Catal. 95 (1985) 578-586.

[314] A. Sasahara, C.L. Pang, H. Onishi, J. Phys. Chem. B 110 (2006) $13453-13457$.

[315] A. Sasahara, C.L. Pang, H. Onishi, J. Phys. Chem. B 110 (2006) 17584-17588.

[316] F. Solymosi, M. Pásztor, J. Phys. Chem. 89 (1985) 4789-4793.

[317] H.F.J. Van't Bilk, J.B.D. Van Zon, T. Huizinga, J.C. Vis, D. C. Koningsberger, R. Prins, J. Phys. Chem. 87 (1983) 2264-2267.

[318] A. Berkó, F. Solymosi, J. Catal. 183 (1999) 91-101.

[319] M. Primet, J. Chem. Soc. Faraday Trans. 74 (1978) 2570-2580.

[320] C.A. Rice, S.D. Worley, C.W. Curtis, J.A. Guin, J. Tarrer, J. Chem. Phys. 74 (1981) 6748.

[321] S. Tsubota, M. Haruta, T. Kobayashi, A. Ueda, A. Nakahara, in: G. Poncelet et al.(Ed.), Preparation of Catalysts V, Elsevier, Amsterdam, 1991, p. 695.

[322] M. Haruta, Catal. Today 36 (1997) 153-166.

[323] T. Akita, M. Okumura, K. Tanaka, K. Ohkuma, M. Kohyama, T. Koyanagi, M. Date, S. Tsubota, M. Haruta, Surf. Interface Anal. 37 (2005) 265-269.

[324] F. Boccuzzi, A. Chiorino, M. Manzoli, P. Lu, T. Akita, S. Ichikawa, M. Haruta, J. Catal. 202 (2001) 256-267.

[325] G.N. Glavee, K.J. Klabunde, C.M. Sorensen, G.C. Hadjipanayis, Inorg. Chem. 34 (1995) 28-35.

[326] E.A. Sterling, J. Stolk, L. Hafford, M. Gross, Metall. Mater. Trans. A 40 (2009) 1701-1709.

[327] B.K. Min, W.T. Wallace, D.W. Goodman, Surf. Sci. 600 (2006) L7-L11.

[328] S. Peters, S. Peredkov, M. Neeb, W. Eberhardt, M. Al-Hada, Surf. Sci. 608 (2013) 129-134.

[329] Turner, V.B. Golovko, O.P.H. Vaughan, P. Abdulkin, A. BerenguerMurcia, M.S. Tikhov, B.F.G. Johnson, R.M. Lambert, Nature 454 (2008) 981-983.

[330] A.M. Kiss, M. Svec, A. Berkó, Surf. Sci. 600 (2006) 3352-3360.

[331] C. Fan, T. Wu, S.L. Anderson, Surf. Sci. 578 (2005) 5-19.

[332] M. Baron, O. Bondarchuk, D. Stacchiola, S. Shaikhutdinov, H.J. Freund, J. Phys. Chem. C 113 (2008) 6042-6049.

[333] P.D. Jadzinsky, G. Calero, C.J. Ackerson, D.A. Bushnell, R. D. Kornberg, Science 318 (2007) 430-433.

[334] M. Zhu, C.M. Aikens, F.J. Hollander, G.C. Schatz, R. Jin, J. Am. Chem. Soc. 130 (2008) 5883-5885.

[335] Z. Wu, De-en Jiang, A.K.P. Mann, D.R. Mullins, Z. Qiao, L.F. Allard, C. Zeng, R. Jin, S.H. Overbury, J. Am. Chem. Soc. 136 (2014) 6111-6122.

[336] K.L. Kelly, E. Coronado, L.L. Zhao, G.C. Schatz, J. Phys. Chem. B 107 (2003) 668-672.

[337] H. Park, Y. Park, W. Kim, W. Choi, J. Photochem. Photobiol. C: Photochem. Rev. 15 (2013) 1-20.

[338] N. Zhang, S. Liu, X. Fu, Y.-J. Xu, J. Phys. Chem. C 115 (2011) 9136-9145.

[339] A.M. Wattson, X. Zhang, R.A. de la Osa, J.M. Sanz, F. Gonsalez, F. Moreno, G. Finelstein, J. Liu, H.O. Everitt, Nano Lett. 15 (2015) 1095-1100.

[340] N. Zettsu, J.M. McLellan, B. Wiley, Y. Yin, Z.-Y. Li, Y. Xia, Angew. Chem. Int. Ed. 45 (2006) 1288-1292.

[341] X. Zhang, P. Li, A. Barreda, Y. Gutierrez, F. Gonsalez, F. Moreno, H. O. Everitt, J. Liu, Nanoscale Horiz. Commun. 1 (2016) 75-80.

[342] C. Clavero, Nat. Photonics 8 (2014) 95-103.

[343] M. Jacob, H. Levanon, P.V. Kamat, Nano Lett. 3 (2003) 353-358.
[344] E. Kowalska, O.O. Mahaney, R. Abe, B. Ohtani, Phys. Chem. Chem. Phys. 12 (2010) 2344-2355.

[345] Y. Tian, T. Tatsuma, Chem. Commun. (2004) 1810-1811.

[346] L. Óvári, L. Bugyi, Zs Majzik, A. Berkó, J. Kiss, J. Phys. Chem. C 112 (2008) 18011-18016.

[347] L. Óvári, A. Berkó, N. Balázs, Zs Majzik, J. Kiss, Langmuir 26 (2010) 2167-2175.

[348] L.Z. Mezey, J. Giber, Jpn. J. Appl. Phys. 21 (1982) 1569-1571.

[349] J. Kiss, L. Óvári, A. Oszkó, G. Pótári, M. Tóth, K. Baán, A. Erdőhelyi, Catal. Today 181 (2012) 163-170.

[350] H.H. Brongersma, H. Draxler, M.B.P. de Ridder, Surf. Sci. Rep. 62 (2007) 63-109.

[351] Vári, L. Óvári, C. Papp, H.-P. Steinrück, J. Kiss, Z. Kónya, Phys. Chem. Chem. Phys. 17 (2015) 5124-5132.

[352] S.A. Tenny, J.S. Ratliff, C.C. Roberts, W. He, S.C. Ammal, A. Heyden, D.A. Chen, J. Phys. Chem. C 114 (2010) 21652-21663.

[353] F. Gao, Y. Wang, D.W. Goodman, J. Phys. Chem. C 114 (2010) 4036-4043.

[354] O. Ozturk, J.B. Park, S. Ma, J.S. Ratliff, J. Zhou, D.R. Mullins, D. A. Chen, Surf. Sci. 601 (2007) 3099-3113.

[355] R. Ferrando, J. Jellinek, R.I. Johnson, Chem. Rev. 108 (2008) 845-910.

[356] J.B. Park, S.F. Conner, D.A. Chen, J. Phys. Chem. C 112 (2008) 5490-5500.

[357] G.C. Bond, Platin. Met. Rev. 51 (2007) 63-68.

[358] M.A. Ghanem, J.P. Sinh, M. Al-Hoshan, A. Al-Suhybani, Electrocatalysis 4 (2013) 134-143.

[359] D. Nepak, S. Darbha, Catal. Commun. 58 (2015) 149-153.

[360] S. Nishimura, Y. Yakita, M. Katayama, K. Higashimine, K. Ebitani, Catal. Sci. Technol. 3 (2013) 351-359.

[361] Z. Konuspayeva, P. Afanasiev, T.-S. Nguyen, L. Di Felice, F. Morfin, N.-T. Nguyen, J. Nelayah, C. Ricolleau, Z.Y. Li, J. Yuan, G. Berhault, L. Piccolo, Phys. Chem. Chem. Phys. 14 (2015) 28112-28120.

[362] L. Jiang, W. Zhu, C. Wang, W. Dong, L. Zhang, G. Wang, B. Chen, C. Li, X. Zhang, Appl. Catal. B 180 (2016) 344-350.

[363] A. Turki, H. Kochkar, C. Guillard, G. Berhault, Appl. Catal. B 138-139 (2013) 401-415.

[364] S.J. Kim, Y.-U. Yun, H.-J. Oh, S.H. Hong, C.A. Roberts, K. Routray, I. E. Wachs, J. Phys. Chem. Lett. 1 (2010) 130-135.

[365] B.C. Viana, O.P. Ferreira, A.G.S. Filho, A.A. Hidalgo, J.M. Filho, O. L. Alves, Vibr. Spect. 55 (2013) 183-187.

[366] A.A. Cavalheiro, A.A. Bruno, J.C. Sacki, M.J. Valente, J.P. S. Florentino, Thin Solid Films 516 (2008) 6240.

[367] G. Wang, Z.Y. Liu, N.J. Wu, Q. Lu, Mater. Lett. 71 (2012) 120-122.

[368] T. Wang, W. Liu, N. Xu, J. Ni, J. Hazard. Mater. 250-251 (2013) 379-386.

[369] J. Thomas, M.-J. Yoon, Appl. Catal. B 111-112 (2012) 502-508.

[370] D. Szieberth, A.M. Ferrari, P. D’Arco, R. Orlando, Nanoscale 3 (2013) 1113-1119.

[371] R. Asahi, T. Morikawa, T.T. Ohwaki, K. Aoki, Y. Taga, Science 293 (2001) 269-271.

[372] A. Fujishima, X. Zhang, D.A. Tryk, Surf. Sci. Rep. 63 (2008) 515-582.

[373] R. Asahi, T. Morikawa, Chem. Phys. 339 (2007) 57-63.

[374] M. Maeda, T. Watanabe, J. Electrochem. Soc. 153 (2006) C186-C189.

[375] I. Bertóti, Catal. Today 181 (2012) 95-101.

[376] B. Buchholcz, H. Haspel, Á. Kukovecz, Z. Kónya, Cryst. Eng. Commun. 16 (2014) 7486-7492.

[377] M. Hodos, E. Horváth, H. Haspel, Á. Kukovecz, Z. Kónya, I. Kiricsi, Chem. Phys. Lett. 399 (2004) 512-515.

[378] Á. Kukovecz, M. Hodos, Z. Kónya, I. Kiricsi, Chem. Phys. Lett. 411 (2005) 445-449.

[379] P. Dong, Y. Wang, B. Liu, L. Guo, Y. Huang, S. Yin, J. Am. Ceram. Soc. 98 (2012) 82-84.

[380] C.-C. Hu, T.-C. Hsu, S.-Y. Lu, Appl. Surf. Sci. 280 (2013) 171-178.

[381] R. Nakamura, T. Tanaka, Y. Nakato, J. Phys. Chem. B 108 (2004) 10617-10620.

[382] N.C. Saha, H.G. Tomkins, J. Appl. Phys. 72 (1992) 3072.

[383] W.H.R. Shaw, J.J. Bordeaux, J. Am. Chem. Soc. 77 (1995) 4729-4733. 
[384] J.-C. Chang, W.-J. Tsai, T.-C. Chiu, C.-W. Liu, J.-H. Chao, C.-H. Lin, J. Mater. Chem. 21 (2011) 4605-4614.

[385] O. Diwald, T.L.T. Thomson, T. Zubkov, E.G. Goralski, S.D. Walck, J. T. Yates Jr., J. Phys. Chem. B 108 (2004) 6004-6008.

[386] Q. Xiang, J. Yu, M. Jaroniec, Phys. Chem. Chem. Phys. 13 (2011) 4853-4861.

[387] B. Buchholcz, E. Varga, T. Varga, J. Kiss, Z. Kónya, Paper presented in 16th Joint Vacuum Conference and 14th European Vacuum Conference, Book of Abstract p. 123. Portoroz, Slovenia, 2016.

[388] J. Kiss, K. Révész, F. Solymosi, Appl. Suf. Sci. 7 (1989) 95-110.

[389] J. Kiss, K. Révész, G. Klivényi, F. Solymosi, Appl. Surf. Sci. 264 (2013) 838-844.

[390] D. Joyner, R.F. Willis, Philos. Mag. A 43 (1981) 815-833.

[391] T.B. Fryberger, J.L. Gland, P.C. Stair, Langmuir 3 (1987) 1015-1025.

[392] D. Chen, D. Yang, Q.W. Wang, Z. Jang, Ind. Eng. Chem. Res. 45 (2006) 4110-4116.

[393] S. Tsubota, T. Nakamura, K. Tanaka, M. Haruta, Catal. Lett. 56 (1998) $131-135$.

[394] T. Akita, K. Tanaka, S. Tsubota, M. Haruta, J. Electron Microsc. 49 (2000) 657-662.

[395] M. Mendez-Cruz, J. Ramirez-Solis, R. Zanella, Catal. Today 166 (2011) $172-179$.

[396] V. Idakiev, Z.Y. Yuan, T. Tabaskov, B.-L. Su, Appl. Catal. A: Gen. 261 (2005) 149-155.

[397] J. Kiss, P. Pusztai, L. Óvári, G. Merza, A. Oszkó, K. Baán, A. Erdőhelyi, Á. Kukovecz, Z. Kónya, Paper Presented at ACSIN-12 Conference, Tsukuba, Japan, 2013.

[398] S.H. Chien, Y.C. Liou, M.C. Kuo, Synth. Met. 152 (2005) 333-336.

[399] S. Li, N. Li, G. Li, L. Li, A. Wang, Y. Cong, X. Wang, G. Xu, T. Zhang, Appl. Catal. B: Environ. 170-171 (2015) 124-134.

[400] C.H. Campos, C.C. Torres, P. Osorio-Vargas, C. Mella, J. Belmar, D. Ruiz, J.L.G. Fierro, P. Reyes, J. Mol. Catal. A: Chem. 398 (2015) 190-202.

[401] G. Chen, F. Xue, Z. Chen, X. Si, X. Zheng, J. Huang, S. Massey, Nano 9 (2014) 1450039-1-1450039-7.

[402] P. Hernandez-Hipolito, N. Juarez-Flores, E. Martinez-Klimova, A. Gomez-Cortes, X. Bokhimi, L. Escobar-Alarcon, T.E. Klimova, Catal. Today 250 (2015) 187-196.

[403] M.N. Chong, B. Jin, C.W. Chow, C. Saint, Water. Res. 44 (2010) 2997-3027.

[404] M. Gratzel, Nature 414 (2001) 338-344.

[405] M. Gratzel, Philos. Trans. R. Soc. A 365 (2007) 993-1005.

[406] B.E. Hardin, H.J. Snaith, M.D. McGehee, Nat. Photonics 6 (2012) $162-169$.

[407] X. Wang, Z. Li, J. Shi, Y. Yu, Chem. Rev. 114 (2014) 9346-9384.

[408] Y. Zhang, Y. Tang, S. Yin, Z. Zeng, H. Zhang, C.M. Li, Z. Dong, Z. Chen, X. Chen, Nanoscale 3 (2011) 4074-4077.

[409] D.H. Kim, J.S. Jang, S.S. Han, K.S. Lee, S.H. Choi, A. Umar, J.W. Lee, D.W. Shin, S.-T. Myung, J.S. Lee, S.-J. Kim, Y.K. Sun, K.S. Lee, J. Phys. Chem. C 113 (2009) 14034-14039.

[410] J.R. Li, Z.L. Tang, Z.T. Zhang, Chem. Mater. 17 (2005) 5848-5855.

[411] S. Suzuki, M. Miyayama, J. Ceram. Soc. Jpn. 118 (2010) 1154-1158.

[412] J.C. Perez-Flores, A. Kuhn, F. Garcia-Alvarado, J. Power Sources 196 (2011) 1378-1385.

[413] J.-Y. Liao, X. Xiao, D. Higgins, G. Lui, Z. Chen, ACS Appl. Mater. Interface 6 (2014) 568-574.

[414] F. Wu, Z. Wang, X. Li, H. Guo, J. Mater. Chem. 21 (2011) $12675-12681$.

[415] J.-H. Kang, S.-M. Paek, J.-H. Choy, Chem. Commun. 48 (2012) $458-460$.

[416] Z. Hong, X. Zheng, X. Ding, L. Jiang, M. Wei, K. Wei, Energy Environ. Sci. 4 (2011) 1886-1891.

[417] T. Dickenson, A. Fiennes, Chemical Kinetics, Pergamon, 1966.

[418] D.K. Lee, H.-W. Shim, J.S. An, C.M. Cho, I.-S. Cho, K.S. Hong, D.W. Kim, Nanoscale Res. Lett. 5 (2010) 1585-1589.
[419] S.Y. Yin, L. Song, X.Y. Wang, Y.H. Huang, K.L. Zhang, Y.X. Zhang, Electrochem. Commun. 11 (2009) 1251-1254.

[420] H. Zhang, G.R. Li, L.P. An, T.Y. Yan, X.P. Gao, H.Y. Zhu, J. Phys. Chem. C 111 (2007) 6143-6148.

[421] K. Kiatkittipong, J. Scott, R. Amal, ACS Appl. Mater. Interface 3 (2011) 3988-3996.

[422] J. Thomas, M. Yoon, Appl. Catal. B: Environ. 111 (2012) 502-508.

[423] M. Mohl, A. Dombovari, E.S. Tuchina, P.O. Petrov, O.A. Bibikova, I. Skovorodkin, A.P. Popov, A.-R. Rautio, A. Sarkar, J.-P. Mikkola, M. Huuhtanen, S. Vainio, R.L. Keiski, A. Prilepsky, A. Kukovecz, Z. Kónya, V.V. Tuchin, K. Kordas, J. Mater. Chem. B 2 (2014) $1307-1316$.

[424] W. Li, C. Liu, Y. Zhou, Y. Bai, X. Feng, Z. Yang, L. Lu, X. Lu, K.Y. Chan, J. Phys. Chem. C 112 (2008) 20539-20545.

[425] G.H. Du, Q. Chen, P.D. Han, Y. Yu, Phys. Rev. B 67 (2003) 035323.

[426] X. Chen, S. Shen, L. Guo, S.S. Mao, Chem. Rev. 110 (2010) 6503-6570.

[427] M.-C. Wu, J. Hiltunen, A. Sapi, A. Avila, W. Larsson, H.C. Liao, M. Huuhtanen, G. Tóth, A. Shchukarev, N. Laufer, A. Kukovecz, Z. Kónya, J.-P. Mikkola, R. Keiski, W.-F. Su, H. Jantunen, K. Kordás, ACS Nano 5 (2011) 5025-5030.

[428] M.C. Wu, H.C. Liao, Y.C. Cho, Hsu, T.H. Lin, W.F. Su, A. Sapi, A. Kukovecz, Z. Kónya, A. Shchukarev, A. Sarkar, W. Larsson, J. P. Mikkola, M. Mohl, G. Toth, H. Jantunen, A. Valtanen, M. Huuhtanen, R. Keiski, K. Kordas, J. Nanopart. Res. 16 (2013) 2143.

[429] A. Sarkar, A. Shchukarev, A.R. Leino, K. Kordas, J.P. Mikkola, P. O. Petrov, E.S. Tuchina, A.P. Popov, M.E. Darvin, M.C. Meinke, J. Lademann, V.V. Tuchin, Nanotechnol 23 (2013) 475711.

[430] T. Umebayashi, T. Yamaki, H. Itoh, K. Asai, J. Phys. Chem. Solids 63 (2002) 1909-1920.

[431] H. Yu, H. Irie, K. Hashimoto, J. Am. Chem. Soc. 132 (2010) 6898-6899.

[432] N. Coleman Jr., S. Perera, E.G. Gillan, J. Solid State Chem. 232 (2015) 241-248.

[433] M. Jovaní, M. Domingo, T.R. Machado, E. Longo, H. Beltrán-Mir, E. Cordoncillo, Dyes Pigment 116 (2015) 106-113.

[434] K.E. Karakitsou, X.E. Verykios, J. Phys. Chem. 97 (1993) 1184-1189.

[435] W. Choi, A. Termin, M.R. Hoffmann, J. Phys. Chem. 98 (1994) 13669-13679.

[436] M.-C. Wu, H.-C. Liao, Y.-C. Cho, G. Toth, Y.-F. Chen, W.-F. Su, K. Kordas, J. Mater. Chem. A 1 (2013) 5715-5720.

[437] A. Fujishima, K. Honda, Nature 238 (1972) 37-38.

[438] A. Kudo, Y. Miseki, Chem. Soc. Rev. 38 (2009) 253-278.

[439] R.M. Navarro Yerga, M.C.A. Galván, F. del Valle, J.A.V. de la Mano, Jos L.G. Fierro, Chem. Sus. Chem. 2 (2009) 471-485.

[440] A. Kudo, Catal. Surv. Asia 7 (2003) 31-38.

[441] K. Maeda, K. Domen, J. Phys. Chem. C 111 (2007) 7851-7861.

[442] K. Maeda, ACS Catal. 3 (2013) 1486-1503.

[443] M. Pirila, R. Lenkkeri, W.M. Goldmann, K. Kordas, M. Huuhtanen, R. L. Keiski, Top. Catal. 56 (2013) 630-636.

[444] M.-S. Wong, W.-C. Chu, D.-S. Sun, H.-S. Huang, J.-H. Chen, P.-J. Tsai, N.-T. Lin, M.-S. Yu, S.-F. Hsu, S.-L. Wang, H.-H. Chang, Appl. Environ. Microbiol. 72 (2006) 6111-6116.

[445] H.A. Foster, I.B. Ditta, S. Varghese, A. Steele, Appl. Microbiol. Biotechnol. 90 (2011) 1847-1868.

[446] P. Wu, R. Xie, K. Imlay, J.K. Shang, Environ. Sci. Technol. 44 (2010) 6992-6997.

[447] A. Kedziora, W. Strek, L. Kepinski, G. Bugla-Ploskonska, W. Doroszkiewicz, J. Sol-Gel Sci. Technol. 62 (2012) 79-86.

[448] P. Calza, C. Hadjicostas, V.A. Sakkas, M. Sarro, C. Minero, C. Medana, T.A. Albanisa, Appl. Catal. B 183 (2016) 96-106.

[449] B. Erjaveca, P. Hudoklin, K. Perc, T. Tisler, M.S. Dolenc, A. Pintar, Appl. Catal. B 183 (2016) 149-158.

[450] R. Žabar, M. Sarakha, A.T. Lebedev, O.V. Polyakova, P. Trebše, Chemosphere 144 (2016) 615-620. 
[451] S. Sakthivel, B. Neppolian, M.V. Shankar, B. Arabindoo, M. Palanichamy, V. Murugesan, Sol. Energy Mater. Sol. Cells 77 (2003) 65-82.

[452] S. Sakthivel, M. Janczarek, H. Kisch, J. Phys. Chem. B 108 (2004) 19384-19387.

[453] A. Houas, H. Lachheb, M. Ksibi, E. Elaloui, C. Guillard, J.M. Herrmann, Appl. Catal. B: Environ. 31 (2001) 145-157.

[454] M.C. Wu, G. Toth, A. Sapi, A.R. Leino, Z. Kónya, A. Kukovecz, W. F. Su, K. Kordas, J. Nanosci. Nanotechnol. 12 (2012) 1244-1421.

[455] I.K. Levy, M. Mizrahi, G. Ruano, G. Zampieri, F.G. Requejo, M. I. Litter, Environ. Sci. Technol. 46 (2012) 2299-2308.

[456] R. Djellabi, M.F. Ghorab, Inst. Chem. E 55 (2015) 1900-1907.

[457] Marta I. Litter, Pure Appl. Chem. 87 (2015) 557-567.

[458] H. Kyung, J. Lee, W. Choi, Environ. Sci. Technol. 39 (2005) $2376-2382$

[459] P. Karthik, R. Vinoth, S.G. Babu, M. Wen, T. Kamegawa, H. Yamashita, B. Neppolian, RSC Adv. 5 (2015) 38759-39752.

[460] Z. Majidnia, A. Idris, Korean J. Chem. Eng. 32 (2015) 1094-1100.

[461] I. Di Sommaa, L. Clarizia, S. Satyro, D. Spasiano, R. Marotta, R. Andreozzi, Chem. Eng. J. 270 (2015) 519-527.

[462] S. Srimala, L. Chin Wei, Z. Syazwani Mohd, Int. J. Photoenergy (2014) 839106.

[463] H. Chen, Y. Shao, Z.Y. Xu, H.Q. Wan, Y.Q. Wan, S.R. Zheng, D. Q. Zhu, Appl. Catal. B 105 (2011) 255-262.

[464] S.Q. Liu, N. Zhang, ACS Appl. Mater. Interface 4 (2012) 6378-6385.

[465] B. O'Regan, M. Graetzel, Nature 353 (1991) 737-740.

[466] J. Halme, P. Vahermaa, K. Miettunen, P. Lund, Adv. Energy Mater. 22 (2010) E210-E234.

[467] S. Feldt, Alternative Redox Couples for Dye-Sensitized Solar Cells (Doctoral thesis), Uppsala University, Sweden, 2013.

[468] S. Mathew, A. Yella, P. Gao, R. H-Baker, B.F.E. Curchod, N. AshariAstani, I. Tavernelli, U. Rothlisberger, Md. K. Nazeeruddin, M. Grätzel, Nat. Chem. 6 (2014) 242-247.

[469] M. Law, L.E. Greene, J.C. Johnson, R. Saykally, P. Yang, Nat. Mater. 4 (2005) 455-459.

[470] G.K. Mor, K. Shankar, M. Paulose, O.K. Varghese, C.A. Grimes, Nano Lett. 6 (2006) 215-218.

[471] A.B.F. Martinson, M.S. Goes, F. Fabregat-Santiago, J. Bisquert, M. J. Pellin, J.T. Hupp, J. Phys. Chem. A 113 (2009) 4015-4021.

[472] M. Adachi, Y. Murata, J. Takao, J. Jiu, M. Sakamoto, F. Wang, J. Am. Chem. Soc. 126 (2004) 14943-14949.

[473] C.J. Lin, W.Y. Yu, S.H. Chien, J. Mater. Chem. 20 (2010) 1073-1077.
[474] B.H. Lee, M.Y. Song, S.Y. Jang, S.M. Jo, S.Y. Kwak, D.Y. Kim, J Phys. Chem. C 113 (2009) 21453-21457.

[475] S.C.T. Lau, J. Dayou, C.S. Sipaut, R.F. Mansa, Int. J. Renew. Energy Res. 4 (2014) 665-674.

[476] J. Sheng, L. Hu, S. Xu, W. Liu, L. Mo, H. Tian, S. Dai, J. Mater. Chem. 21 (2011) 5457.

[477] S.D. Stranks, G.E. Eperon, G. Grancini, C. Menelaou, M.J.P. Alcocer, T. Leijtens, L.M. Herz, A. Petrozza, H.J. Snaith, Science 342 (2013) 341-344.

[478] N.-G. Park, J. Phys. Chem. Lett. 4 (2013) 2423-2429.

[479] H.S. Kim, S.H. Im, N.G. Park, J. Phys. Chem. C 118 (2014) 5615-5625.

[480] Y. Ogomi, A. Morita, S. Tsukamoto, T. Saitho, Q. Shen, T. Toyoda, K. Yoshino, S.S. Pandey, T.L. Ma, S. Hayase, J. Phys. Chem. C 118 (2014) 16651-16659.

[481] K. Manseki, T. Ikeya, A. Tamura, T. Ban, T. Sugiura, T. Yoshida, RSC Adv. 4 (2014) 9652-9655.

[482] C.-Y. Chang, C.-Y. Chu, Y.-C. Huang, C.-W. Huang, S.-Y. Chang, C.A. Chen, C.-Y. Chao, W.-F. Su, ACS Appl. Mater. Interfaces 7 (2015) 4955-4961.

[483] 〈〈http://www.nrel.gov/ncpv/images/efficiency_chart.jpg $\rangle$.

[484] Z. Liu, V. Subramania, M. Misra, J. Phys. Chem. C 113 (2009) $14028-14033$.

[485] D. Kuang, J. Brillet, P. Chen, M. Takata, S. Uchida, H. Miura, K. Sumioka, S.M. Zakeeruddin, M. Grätzel, ACS Nano 2 (2008) $1113-1116$

[486] M. Toivola, J. Halme, K. Miettunen, K. Aitola, P.D. Lund, Int. J. Energy Res. 33 (2009) 1145-1160.

[487] R. Xu, J. Li, A. Tan, Z. Tang, Z. Zhang, J. Power Sources 196 (2011) 2283-2288.

[488] H. Zhang, X.P. Gao, G.R. Li, T.Y. Yan, H.Y. Zhu, Electrochim. Acta 53 (2008) 7061-7068

[489] B.L. Wang, Q. Chen, J. Hu, H. Li, Y.F. Hu, L.M. Peng, Chem. Phys. Lett. 406 (2005) 95-100.

[490] J. Chen, L. Yang, S. Fang, Y. Tang, Electrochim. Acta 55 (2010) 6596-6600.

[491] A. Guerfi, P. Charest, K. Kinoshita, M. Perrier, K. Zaghib, J. Power Sources 126 (2004) 163-168.

[492] V.S. Hernandez, L.M.T. Martinez, G.C. Mather, A.R. West, J. Mater. Chem. 6 (1996) 1533-1536.

[493] J.-Y. Liao, X. Xiao, D. Higgins, G. Lui, Z. Chen, ACS Appl. Mater. Interfaces 6 (2014) 568-574. 